Document downloaded from:

http://hdl.handle.net/10251/140156

This paper must be cited as:

Puga, AV. (2016). Photocatalytic production of hydrogen from biomass-derived feedstocks. Coordination Chemistry Reviews. 315:1-66. https://doi.org/10.1016/j.ccr.2015.12.009

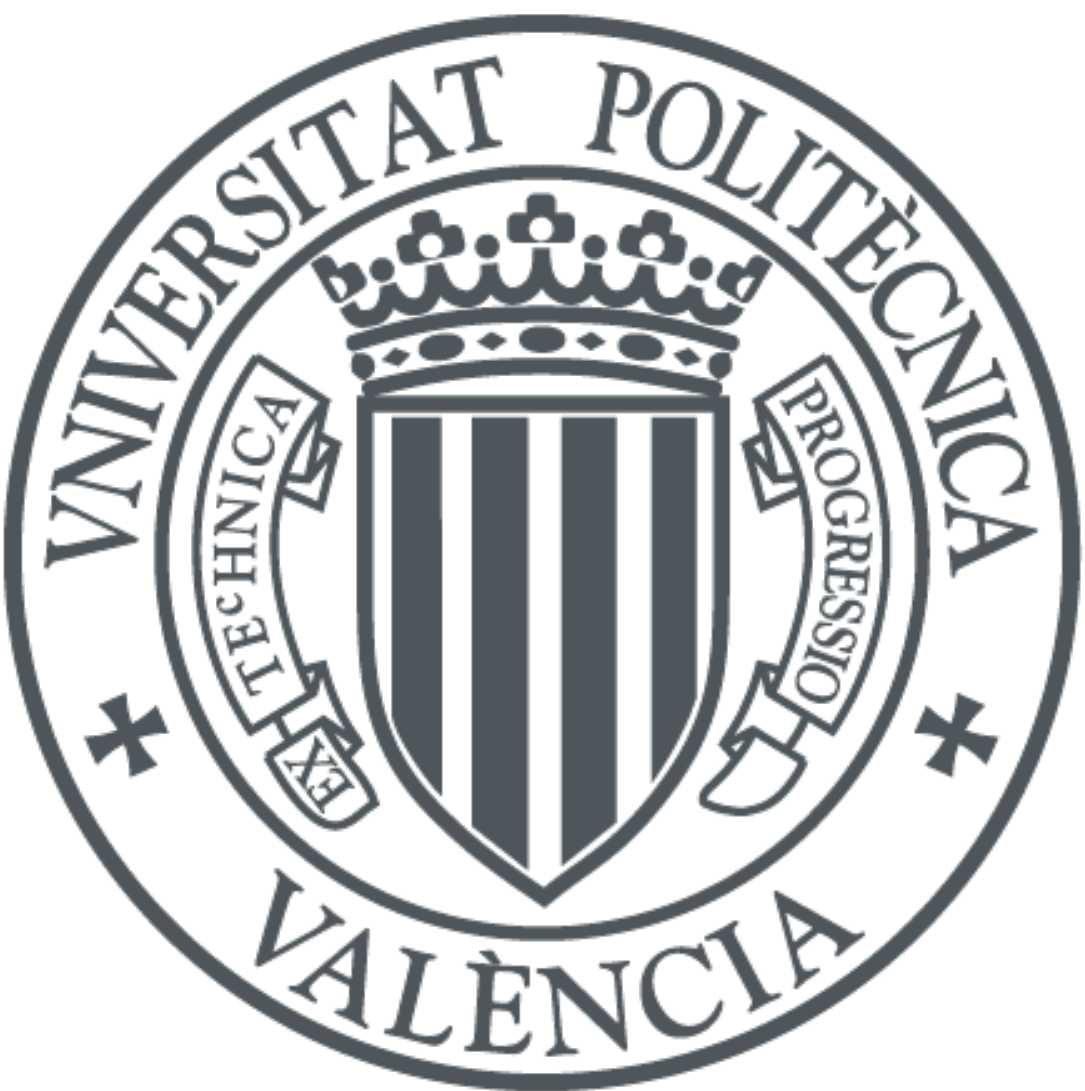

The final publication is available at

https://doi.org/10.1016/j.ccr.2015.12.009

Copyright Elsevier

Additional Information 


\title{
Photocatalytic production of hydrogen from biomass-derived feedstocks
}

\author{
Alberto V. Puga* \\ Instituto Universitario de Tecnología Química CSIC-UPV, Universitat Politècnica de València, Av. \\ De los Naranjos, s/n, 46022 Valencia, Spain \\ E-mail:a.puga@csic.es
}

\section{Contents}

1. Introduction

2. Photocatalytic reforming in the context of biomass-to-fuels technologies

2.1. Liquid fuels from biomass

2.2. Hydrogen from biomass

2.2.1. Reforming of biomass derivatives

2.3. Photocatalytic reforming of oxygenates-Photoreforming

2.3.1. Scope: Biomass resources and biomass-derived oxygenated substrates for photocatalytic $\mathrm{H}_{2}$ production

3. Reaction pathways, thermodynamics and energy balances

3.1. Thermodynamics of reforming and of competitive reactions

3.2. Energy balances and heats of combustion

3.3. Efficiency of radiative energy use

4. Mechanism

4.1. Photo-generation of charges upon light absorption

4.1.1. Time-resolved spectroscopic evidence for transient photo-generated electrons

4.1.2. Time-resolved spectroscopic evidence for transient photo-generated holes

4.2. Adsorption-dependent kinetics

4.2.1. Postulation of adsorption-dependent models

4.2.2. Suitability and utility of kinetic models

4.3. Oxidation mechanisms

4.3.1. Comparison of aerobic photo-oxidation and anaerobic photoreforming

4.3.2. Direct $v s$. indirect oxidation

4.4. The speciation of adsorbed substrates and intermediates

4.4.1. Speciation of adsorbed substrates

4.4.2. In situ spectroscopic monitoring under irradiation

4.5. Postulation of different mechanistic routes

4.5.1. Alcohol photoreforming mechanisms

4.5.2. Carboxylic acid photoreforming mechanisms

4.5.3. Proposal of a consolidated photoreforming mechanism

4.6. Deactivation events

5. Photocatalysts for $\mathrm{H}_{2}$ production from oxygenated substrates

5.1. Photocatalysts based on $\mathrm{TiO}_{2}$

5.1.1. Synthesis of $\mathrm{TiO}_{2}$ materials with different phase structures

5.1.2. Synthesis of $\mathrm{TiO}_{2}$ materials with different morphologies

5.1.3. Phase junction engineering in $\mathrm{TiO}_{2}$ photocatalysts 
5.1.4. Heterojunction photocatalysts based on $\mathrm{TiO}_{2}$

5.1.5. Doped $\mathrm{TiO}_{2}$ for enhanced visible light absorption

5.2. Photocatalysts based on oxides other than $\mathrm{TiO}_{2}$

5.2.1. Simple oxides other than $\mathrm{TiO}_{2}$

5.2.2. Mixed oxides

5.3. Photocatalysts based on sulfides and other chalcogenides

5.3.1. Cadmium sulfide

5.3.2. Sulfides other than CdS

5.3.3. Other chalcogenides

5.4. Photocatalysts based on novel semiconductors

5.4.1. Carbon nanomaterials

5.4.2. Metal-organic frameworks (MOFs) and covalent-organic frameworks (COFs)

5.4.3. Polymeric semiconductors

5.5. Co-catalyst engineering for $\mathrm{H}_{2}$ production

5.5.1. Metal or metal oxide co-catalysts

5.5.2. Metal sulfide or other non-oxide co-catalysts

5.5.3. Nanocarbon-based co-catalysts

5.5.4. Molecular metal complexes as co-catalysts and organic sensitisers

6. Effect of reaction conditions on photoreforming efficiency

6.1. Effect of light sources and irradiance

6.1.1. Light sources

6.1.2. Photo-action spectra

6.1.3. Effect of light intensity

6.2. Effect of temperature

6.3. Effect of $\mathrm{pH}$

6.4. Effect of substrate concentration

6.5. Effect of catalyst concentration

7. Biomass-derived substrates for hydrogen production by photoreforming

7.1. Photoreforming of alcohols

7.1.1. Methanol

7.1.2. Ethanol

7.1.3. Glycerol and other polyols

7.1.4. Comparison to other alcohols and polyols

7.2. Photoreforming of aldehydes

7.2.1. Formaldehyde

7.2.2. Acetaldehyde

7.2.3. Other aldehydes and ketones

7.3. Photoreforming of carboxylic acids

7.3.1. Formic acid

7.3.2. Acetic acid

7.3.3. Lactic acid

7.3.4. Other acids

7.4. Photoreforming of saccharides

7.4.1. Monosaccharides 


\subsubsection{Disaccharides}

7.4.3. Polysaccharides

7.5. Raw biomass feedstocks for photocatalytic $\mathrm{H}_{2}$ production

7.6. Photocatalytic decontamination of waste streams with concomitant $\mathrm{H}_{2}$ production

8. Conclusions and prospects 


\begin{abstract}
The application of photocatalysis for the utilisation of sunlight energy is intensely investigated in present times, particularly in prospect of generating solar fuels by hydrogen production or $\mathrm{CO}_{2}$ reduction processes as tools for societies aiming to relief their thirst for fossil resources. From the perspective of sustainability, the rational use of biomass-derived feedstocks for photocatalytic $\mathrm{H}_{2}$ production is a feasible, proven and highly efficient process. In this review, in addition to delving into physico-chemical fundamentals of photocatalytic processes on semiconductors, the research activity on this topic related to design of revolutionary semiconductor-based materials, generally including metallic nanoparticles or complexes as hydrogen-evolving co-catalysts, is outlined and critically evaluated. Moreover, the use of sunlight and renewable feedstocks for the generation of hydrogen, as a compelling opportunity for the energy sector, is emphasised. Special focus is also set on the valorisation of biorefinery products, agricultural residues and industrial or municipal waste.
\end{abstract}

\title{
Keywords
}

Photocatalysis

Solar energy

Biomass

Hydrogen

Semiconductors 


\section{Introduction}

The success of future energy schemes is expected to heavily depend on the rational transition from the exploitation of fossil fuels to the efficient use of renewable energies. The judicious transformation of biomass resources into fuels appears as a convenient strategy in these terms, given the rich chemistry developed in recent years and the suitability of the thereby generated biofuels to fit current power and transportation infrastructures [1]. Moreover, sunlight is regarded as the ultimate source of energy for any long-term sustainable future [2]. Its storage in the form of solar fuels is one of the most desirable options [3]. Among such strategies, solar production of hydrogen as a clean energy carrier is particularly compelling [2b, 2c, 4]. The design of photocatalytic or photoelectrocatalytic systems has enabled the realisation of overall water splitting in one step, yet at low efficiencies under visible light - hardly surpassing $5 \%$ for the best performers [5]. Discouraging as they may appear, these are excellent values since they pave the way to a direct solar-to-fuel scenario whereby sunlight energy is stored in the form of chemical energy. The intensive investigations on this topic have impressively enriched the knowledge of photocatalysis as a feasible technology for $\mathrm{H}_{2}$ generation [6]. In addition to overall water splitting, photocatalytic reforming - photoreforming — has emerged as a highly efficient light-promoted production of $\mathrm{H}_{2}$ from oxygenated organic substances and water [7]. In this review, fundamental and practical aspects of these transformations regarding the use of biomass derivatives as the substrates are discussed.

Photoreforming can be viewed as an intermediate process between photocatalytic water splitting and photo-oxidation, as illustrated in the following example. If one employs an archetypal photocatalyst such as $\mathrm{Pt} / \mathrm{TiO}_{2}$ for overall water splitting, production of $\mathrm{H}_{2}$ and $\mathrm{O}_{2}$ may be observed under appropriate conditions, albeit at low quantum yields due to slow rates, back-reactions, and deactivation events $[5 \mathrm{~d}, 7 \mathrm{e}, 8]$. Furthermore, let us recall that water dissociation is an endergonic ( $\triangle G^{0}=237.1 \mathrm{~kJ} \mathrm{~mol}^{-1}$ ) transformation and thus a thermodynamic barrier is also at play. If the same $\mathrm{Pt} / \mathrm{TiO}_{2}$ photocatalyst is used in a completely different aqueous medium containing an oxygenated organic substance (let us consider ethanol as a model) under aerobic conditions oxidation of the substrate would take place readily. Even bare titanium dioxide would perform satisfactorily. The overall spontaneous process (photo-oxidation of ethanol, $\triangle G^{0}=-1325.4 \mathrm{~kJ} \mathrm{~mol}^{-1}$ ), is a favourable photocatalytic one on irradiated $\mathrm{Pt} / \mathrm{TiO}_{2}$. The behaviour of the same system in an intermediate situation, whereby the reaction is performed in the presence of ethanol but in the absence of oxygen, would lead to a combination of the outcomes of the two processes described above. Precisely speaking, ethanol would be also oxidised on titanium dioxide surfaces, whereas water would be 
reduced on platinum (see Figure 1 for a generic illustration). The overall transformationphotoreforming of ethanol-is marginally endergonic $\left(\triangle G^{0}=97.4 \mathrm{~kJ} \mathrm{~mol}^{-1}\right)$ and takes place at noticeably high rates on materials of this kind, yielding $\mathrm{H}_{2}$ as a product containing the entire amount of free energy (and heat) content of the starting substrate. It is worth noting that also a portion of (excess) solar energy is stored in the form of chemical energy in hydrogen [7b, 9].

As inferred from the examples described above and illustrated in Figure 1, the transformation of oxygenated organic substances by photocatalytic reforming-photoreforming-makes the production of $\mathrm{H}_{2}$ possible with the only energy input of light irradiation. Moreover, if the substrates are derived from biomass, only virtually renewable feedstocks are consumed [1a, 1c, 1d, 10]. As set forth in this review, the large amount of research on the photoreforming of biomass-derived substrates published during the past three-to-four decades has demonstrated the feasibility of this technology. Interestingly, the selectivity of the photocatalytic reforming processes outperforms that of the thermo-catalytic analogues, owing to the milder (ambient) conditions under which the former proceeds, with comparable activities.

The first part of this review summarises state-of-the-art in biomass-to-fuels conversion, and in a dedicated section, a specific overview of biomass-to-hydrogen routes sets out the scene for comparison of photoreforming with other contenders. Next, an account of thermodynamic aspects is conveyed. This discussion is exemplified by pondering free energies, enthalpies and calorific powers of both substrates and derived $\mathrm{H}_{2}$. The following part of the review is dedicated to the study of the underlying mechanisms by independently considering the main steps of the overall photoreforming transformation. A considerable focus on this overview is then devoted to the design, preparation, characterisation and performance of active materials, reflecting the enormous effort dedicated by a plethora of research groups to fabricate semiconductor-based photocatalysts including metallic nanoparticles or complexes as co-catalysts, with the optimum characteristics for efficient light absorption and utilisation. After a systematic examination of reaction conditions on photoreforming outcomes, the last part of this review outlines the different feasible biomass derivatives for $\mathrm{H}_{2}$ generation by this procedure. The availability, energy balance, suitability and reactivity of each class of feedstocks are therein reviewed. The motivation of using raw (or gently processed) biomass and waste streams for their valorisation or remediation, respectively, with concomitant production of $\mathrm{H}_{2}$ as an energy carrier, will be analysed. 


\section{Photocatalytic reforming in the context of biomass-to-fuels technologies}

\subsection{Liquid fuels from biomass}

Plant-derived biomass has been a source of energy for humankind since the Lower Palaeolithic. In fact, the controlled combustion of wood to produce heat and light constituted the first chemical process to be mastered by the human species, enabling further transformations such as cooking or pottery, and thus leading to a genuine technological revolution [2b]. Until the end of the eighteenth century, wood and charcoal were the main sources of energy [11]. Thereafter, their production could no longer meet the growing demand and the increasingly challenging technological requirements of industry and transport, which were satisfied by the large-scale exploitation of mineral resources, that is, the inexpensive, stable and easily transportable fossil fuels. However, it is widely accepted that fossil reserves are finite and their exploitation will become more difficult in the near future [2a]. This situation is urgently calling for alternative strategies. In this context, the utilisation of biomass for the production of fuels which can be employed in current power generation systems and transportation engines is gaining attention [1a-d, 1g].

The transformation of the organic matter present in plants offers many possibilities for the production of fuels [1a-d]. First-generation biofuels (biodiesel and bio-ethanol), and are currently produced commercially in large quantities from dedicated crops [1a, 1b, 1d, 1g, 11], yet they are often derived from edible feedstock, and require extensive arable land use, thus jeopardising food supply and proving counter-productive regarding carbon dioxide emissions [12]. These controversial issues have prompted a great deal of research into second-generation biofuels produced from lignocellulosic biomass. Given their chemical complexity, transformation of lignocelluloses requires harsh conditions (pyrolysis or gasification) or costly catalysts (enzymatic hydrolysis/fermentation) [1a, 1d, 1g, 11].

\subsection{Hydrogen from biomass}

In the quest of less polluting fuels, molecular hydrogen gas (dihydrogen, $\mathrm{H}_{2}$ ) is considered as one of the most promising energy carriers [ $4 \mathrm{~b}, 4 \mathrm{~d}, 13]$, provided it is obtained from renewable resources. The importance of hydrogen in fuel cells for clean energy generation can be coupled in this manner with the utilisation of renewable feedstocks. However, $\mathrm{H}_{2}$ is mostly produced from non-renewable fossil fuels [14]. As a viable alternative, hydrogen can be obtained from biomass by various chemical procedures [1a, 1g, 14-15], which can be divided into two generic classes: $(i)$ direct gasification and (ii) indirect transformation of biomass into platform chemicals and subsequent reforming. These different routes are schematically depicted in Figure 2. Direct gasification of raw lignocellulosic materials produces mostly $\mathrm{H}_{2}$ and $\mathrm{CO}$ [1a, 1d, 14-15, 16]. The indirect 
transformation strategy can be achieved by a number of routes, whereby the first step can be based on either pyrolysis or hydrolysis leading to monomeric (or oligomeric) oxygenated substances which would be in turn reformed into a gaseous stream rich in $\mathrm{H}_{2}$ and $\mathrm{CO}_{2}[1 \mathrm{a}, 14-15,17]$.

As mentioned above, gasification involves the one-step conversion of biomass - either raw or scantly pretreated by mechanical means - under high temperatures (typically $750-1000{ }^{\circ} \mathrm{C}$ ) in the presence of oxygen and/or steam, to yield a mixture containing $\mathrm{H}_{2}, \mathrm{CO}$ and variable amounts of $\mathrm{CO}_{2}, \mathrm{CH}_{4}$ and other gaseous substances can also be generated, in addition to solid by-products such as char [1a, 1d, 14-15, 16]. Gasification is a versatile technology which can be adapted for a wide range of biomass feedstocks, although it bears the limitation of the extremely harsh conditions involved.

2.2.1. Reforming of biomass derivatives. In the second set of transformations, the indirect routes, the starting biomass can be processed in a first step to yield liquid streams whereby the initially cross-linked, high molecular weight biopolymers (cellulose, hemicelluloses and lignin) are deconstructed into mixtures of lower molecular weight oxygenated chemical entities. The main procedures in this context are pyrolysis (leading to the production of bio-oils, complex mixtures of oxygenated substances, as detailed in Table 1), and hydrolysis [1a, 1d, 1g, 16].

Reforming of bio-oils or bio-oil fractions in the presence of water (liquid or in the form of steam) under a wide variety of process conditions can be carried out yielding $\mathrm{H}_{2}$-rich gaseous streams. The process is usually performed at high temperatures $\left(\mathrm{ca} .850^{\circ} \mathrm{C}\right)$ and short residence times on supported nickel or noble metal catalysts [18]. A different methodology consists in the liquid aqueous phase reforming under milder conditions $\left(250-350^{\circ} \mathrm{C}\right)[17 \mathrm{~b}]$. In either case, the temperatures are too high for the reforming reaction to proceed selectively. Most compounds containing functional groups such as carbonyls or carboxylic acids may undergo a range of degradation (e.g. condensations or cyclisations), whereas saccharides are prone to decompose by several routes including dehydration or polymerisation. As a result, frequent problems in thermocatalytic reforming of bio-oils are the formation of carbonaceous deposits which cause catalyst deactivation, the production of high amounts of $\mathrm{CO}$, or the pyrolysis of saccharides even prior to contact with the catalyst bed [18]. However, reforming of pyrolysis oils for the production of $\mathrm{H}_{2}$ appears as one of the most reliable options for the valorisation of such unstable and complex biomass products. 
Table 1. Typical composition ranges of pyrolysis oils ${ }^{a}$

\begin{tabular}{|c|c|}
\hline substance & wt. $\%$ \\
\hline water & $5-35$ \\
\hline char & $6-32$ \\
\hline gases & $7-30$ \\
\hline carboxylic acids & \\
\hline acetic acid & $0.5-12.0$ \\
\hline formic acid & $0.5-9.1$ \\
\hline propanoic acid & $0.1-1.7$ \\
\hline other acids & $<1$ \\
\hline saccharides & \\
\hline levoglucosan & $1.2-15.0$ \\
\hline cellobiose & $0.6-3.2$ \\
\hline fructose & $0.7-2.9$ \\
\hline xylose & $0.1-1.4$ \\
\hline glucose & $0.4-1.3$ \\
\hline aldehydes and ketones & \\
\hline glycolaldehyde & $0.9-13.0$ \\
\hline acetaldehyde & $1.0-8.5$ \\
\hline hydroxyacetone & $0.7-7.1$ \\
\hline glyoxal & $0.9-4.6$ \\
\hline formaldehyde & $1.4-3.3$ \\
\hline lactaldehyde & $0.9-2.2$ \\
\hline alcohols and polyols & \\
\hline methanol & $0.4-2.4$ \\
\hline ethylene glycol & $0.8-2.2$ \\
\hline ethanol & $0.6-1.4$ \\
\hline furanic derivatives & \\
\hline furfuryl alcohol & $0.7-7.4$ \\
\hline 5-(hydroxymethyl)furfural & $0.3-2.2$ \\
\hline furfural & $0.3-1.1$ \\
\hline aromatic compounds & \\
\hline insoluble lignin & $21-34$ \\
\hline isoeugenol & $0.1-4.6$ \\
\hline phenol & $0.1-3.8$ \\
\hline 4-methylguaiacol & $0.4-1.9$ \\
\hline syringaldehyde & $0.1-1.5$ \\
\hline
\end{tabular}

${ }^{a}$ Based on the set of data compiled in references [19] and [20] for pyrolysis oils obtained from different types of wood (e.g. oak, poplar, aspen, white spruce, red maple, hog fuel or pine) and in different reactor configurations (e.g. fluidized bed, updraft gasifier, vortex reactor, vacuum pyrolysis reactor) and at different temperatures $\left(450-650{ }^{\circ} \mathrm{C}\right)$; please note that these variable conditions account for the scattered data.

Another important indirect biomass-to-hydrogen pathway is based on the aqueous phase reforming of various oxygenated substrates derived from lignocellulose hydrolysis and subsequent transformations (see Figure 2). This strategy is supported by previous studies on the catalytic aqueous phase reforming of a variety of model oxygenates [21]. Dumesic and co-workers reported that the selectivity of the process can be adjusted by the use of appropriate catalysts. In this line, $\mathrm{H}_{2}$ production is favoured and that of alkanes is minimised with $\mathrm{Pt}, \mathrm{Pd}$ or Ni-Sn catalysts, preferably supported on $\mathrm{Al}_{2} \mathrm{O}_{3}$ [17c]. These catalysts display high activities for $\mathrm{C}-\mathrm{C}$ scission, dehydrogenation and water-gas shift reactions, while keeping a low extent of side reactions such as $\mathrm{C}-\mathrm{O}$ scission or methanation. 
In summary, two-stage processes based on, first, the processing of lignocellulosic biomass yielding light oxygenates, and subsequently the aqueous phase reforming of these oxygenates to produce $\mathrm{H}_{2}$, proceed at considerably milder conditions than the one-stage treatments (gasification) and have the potential of becoming interesting routes in the biomass-to-hydrogen scenario. Nonetheless, thermochemical processes are energy intensive, often requiring high temperatures and pressurised systems, and usually lead to degradation or side reactions which have detrimental effects on $\mathrm{H}_{2}$ selectivities (50-60\% of the theoretical maximum) and formation of considerable amounts of alkanes are formed $[17 \mathrm{a}, 17 \mathrm{c}]$. Therefore, the quest for processes enhancing the reforming reaction (Equation 1 below) and limiting any other transformations is still needed, and relevant success in this field would certainly prove beneficial for biomass-to-hydrogen technologies.

$\mathrm{C}_{x} \mathrm{H}_{y} \mathrm{O}_{z}+(2 x-z) \mathrm{H}_{2} \mathrm{O} \rightarrow(2 x+y / 2-z) \mathrm{H}_{2}+x \mathrm{CO}_{2}$

\subsection{Photocatalytic reforming of oxygenates-Photoreforming}

The generally overlooked option of using light to promote the reforming of oxygenates to $\mathrm{H}_{2}$ and $\mathrm{CO}_{2}$ on the appropriate photocatalytic materials bears great potential to drive future improvements. This is based on the efficient aqueous phase reforming of a range of oxygenated substrates (which can be derived from biomass) in suspensions of semiconductor-based photocatalysts, as observed by several researchers. The photocatalytic reactions proceed at ambient or near-ambient temperatures $\left(20-60^{\circ} \mathrm{C}\right)$, and thus, the extent of degradation reactions is negligible, resulting in high $\mathrm{H}_{2}$ selectivities according to Equation 1 [9]. This represents a clear advantage over the thermocatalytic reforming processes [22]. The deep knowledge of such materials in this field is reminiscent of their widespread use as photocatalysts [7e, 23]. Regarding the ultimate goal of utilising solar light for the production of $\mathrm{H}_{2}$ from biomass, Kondarides and co-workers proved that simulated sunlight can drive the complete transformation of model biomass-derived oxygenates according to equation (1) on $\mathrm{Pt} / \mathrm{TiO}_{2}$ photocatalysts without deactivation [9, 24]. Furthermore, the reforming of some oxygenates is endergonic, a fact which indicates that to a certain extent, solar energy can be stored as chemical energy in the generated $\mathrm{H}_{2}$, and that the total heat content of the fuel can be increased relative to that of the starting substrate (see Section 3 below) [7b, 9]. More interestingly, the reported activities of the photocatalysts utilised for this reaction are remarkably high, in the order of tens of thousands of micromoles per hour per gram of catalyst (> $10^{4} \mu \mathrm{mol} \mathrm{g}_{\mathrm{cat}}{ }^{-1} \mathrm{~h}^{-1}$ ) [7e]. Based on the high activity, versatility and energy efficiency of the photocatalytic reforming of biomass-derived oxygenates, this technology bears great potential to widen the portfolio of processes for the straightforward generation of hydrogen. In addition, the feedstocks involved are inexpensive and solar light can be used as the only energy source. 
Despite the efficiency of photoreforming, this field of research has not received the attention that it deserves for the production of $\mathrm{H}_{2}$, and only a limited number of overviews of this area of research have been published. Cargnello et al. summarised prior art on the photocatalytic reforming of methanol, ethanol, glycerol and sugar molecules [7b]. An overview covering a similar scope of processes by Shimura and Yoshida also included thermodynamic considerations [7d]. Melo and Silva summarised results on photoreforming of biomass derivatives and established a comparison with enzymatic and thermocatalytic competitor processes [25]. The group of Bowker dedicated significant efforts to study the photoreforming of methanol, mainly focusing on the activity and mechanistic aspects related to the use of titania-supported metal catalysts [26]. A review by Rossetti was also devoted to compile the advances in the field, including the area of $\mathrm{H}_{2}$ production from organic pollutants in water [27]. Specific accounts on copper- [28] or gold-modified [29] $\mathrm{TiO}_{2}$ photocatalysts or on the photoelectrochemical production of $\mathrm{H}_{2}$ using biomass-derived substrates [30] are also available. Transformation of oxygenated substrates by the action of light-promoted processes should not only be contemplated from the perspective of photoreforming for $\mathrm{H}_{2}$ production, yet also more widely, as a set of opportunities in the field of organic synthesis incorporating elementary oxidative steps (e.g. alcohol to aldehyde) in the absence of oxidising agents [31].

\subsubsection{Scope: Biomass resources and biomass-derived oxygenated substrates for} photocatalytic $\mathbf{H}_{2}$ production. Biomass offers an enormous potential with regard to the number and amounts of valuable oxygenated substances that can be obtained from the many feedstocks available. Among these, the most important materials for transformation into fuels and chemicals can be classified into the following generic types: (i) lignocelluloses, (ii) starch- and sugar-based crops, (iii) vegetable oil crops [1a]. The most abundant are lignocellulosic materials, since they represent a large proportion of all plant mass in the biosphere, forming the cell walls and connective tissues of their structural parts. Lignocellulosic material can be obtained from a variety of sources, including forest products (wood, timber industry residues or shrubs), agricultural by-products (corn hobs, rice husks or wheat straw), waste materials (urban solid waste, paper scrap or used cardboard), or energy crops (sorghum, switchgrass or water hyacinth). Crops producing starch or sugar (chiefly maize and sugar cane, respectively) are more appropriate for biofuel production than lignocelluloses from an economical point of view, although they are edible, and thus their use is controversial due to interference with food prices and arable land use. A similar situation is related to vegetable oil production involving crops such as rapeseed or oil palm. 
The main families of such oxygenates are schematically presented in Figure 3. Their sources, and related transformation processes resulting in their formation are also shown. It is clearly shown there that biomass-derived substances bear a wide variety of oxygen-containing functionalities, including hydroxyl groups (alcohols, polyols, phenols), carbonyls (aldehydes or ketones), carboxylic moieties (present in organic acids), acetals (formed by intramolecular cyclisations in sugars) or ethers (for example, in lignin derivatives such as guaiacols). Some molecules contain several kinds of functional groups (e.g. lactic acid, containing both a hydroxyl group and a carboxylic acid).

The use of alcohols such as methanol or ethanol as sacrificial electron donors is common practice for the photocatalytic $\mathrm{H}_{2}$ production from aqueous media [7e]. Activities are indeed increased by operating in this fashion. However, this complicates literature searches in the topic under review herein, since in many occasions, reports about photocatalytic $\mathrm{H}_{2}$ production are titled using the generic term "water splitting" in journal articles, whilst in reality considerable amounts of methanol or ethanol are used in the aqueous liquid phase. Herein, those research works dealing with photocatalytic hydrogen generation from oxygenated organic substrates, mostly derived from biomass, have been taken into account.

The occurrence of varied oxygenated functionalities mentioned above (and shown in Figure 3) in biomass-derived feedstocks is responsible for the marked photoreforming reactivity of such substrates. The production of $\mathrm{H}_{2}$ can be achieved in this way at remarkably high rates from a variety of substances obtained from renewable resources, and more importantly, the feedstocks can be chosen from waste materials, thus opening a route for their valorisation. Ultimately, the process can be implemented for the sunlight-driven generation of $\mathrm{H}_{2}$ from inexpensive feedstocks.

\section{Reaction pathways, thermodynamics and energy balances}

\subsection{Thermodynamics of reforming and of competitive reactions}

The reforming of oxygenated organic molecules $\left(\mathrm{C}_{x} \mathrm{H}_{y} \mathrm{O}_{z}\right.$ see equation 1) entails their conversion into $\mathrm{H}_{2}$ and $\mathrm{CO}_{2}$ in the presence of water. The entire transformation may be complex, consisting of several steps, mainly dehydrogenation, $\mathrm{C}-\mathrm{C}$ scission, $\mathrm{C}-\mathrm{O}$ scission and water-gas shift reactions. It is analogous to the steam reforming of hydrocarbons, the most important example being the steam reforming of methane (equation 2), which accounts for more than $90 \%$ of the hydrogen gas produced worldwide [4d, 13-15]. Steam reforming generally takes place via two main steps, the first one yielding $\mathrm{CO}$, which is further transformed to $\mathrm{CO}_{2}$ in the second step via the water-gas shift 
reaction (equations 3 and 4 in the case of methane, respectively). While CO formation (equation 3) is endergonic, the water-gas shift (equation 4) is slightly exergonic, resulting in a non-spontaneous global process (equation 2) under standard conditions [17c].

$$
\begin{array}{ll}
\mathrm{CH}_{4(\mathrm{~g})}+2 \mathrm{H}_{2} \mathrm{O}_{(\mathrm{l})} \leftrightarrows 4 \mathrm{H}_{2(\mathrm{~g})}+\mathrm{CO}_{2(\mathrm{~g})} & \triangle G^{0}=130.7 \mathrm{~kJ} \mathrm{~mol}^{-1} \\
\mathrm{CH}_{4(\mathrm{~g})}+\mathrm{H}_{2} \mathrm{O}_{(\mathrm{l})} \leftrightarrows 3 \mathrm{H}_{2(\mathrm{~g})}+\mathrm{CO}_{(\mathrm{g})} & \triangle G^{0}=150.8 \mathrm{~kJ} \mathrm{~mol}^{-1} \\
\mathrm{CO}_{(\mathrm{g})}+\mathrm{H}_{2} \mathrm{O}_{(\mathrm{l})} \leftrightarrows \mathrm{H}_{2(\mathrm{~g})}+\mathrm{CO}_{2(\mathrm{~g})} & \triangle G^{0}=-20.1 \mathrm{~kJ} \mathrm{~mol}^{-1}
\end{array}
$$

In the case of the simplest oxygenated counterpart of methane, i.e. methanol, the reforming process is considerably less energy-demanding. The standard free energy change for methanol reforming (equation 5) is marginally positive.

$$
\mathrm{CH}_{3} \mathrm{OH}_{(\mathrm{l})}+\mathrm{H}_{2} \mathrm{O}_{(\mathrm{l})} \leftrightarrows 3 \mathrm{H}_{2}^{(\mathrm{g})}+\mathrm{CO}_{2(\mathrm{~g})} \quad \triangle G^{0}=9.3 \mathrm{~kJ} \mathrm{~mol}^{-1}
$$

This is theoretically illustrated in Figure 4, where the free energy changes are plotted against temperature for conditions near those employed for typical gas phase methane reforming [32]. The reaction would only proceed spontaneously above $766 \mathrm{~K}$ for methane, whereas methanol reforming would be clearly favoured at that temperature, and even at ambient or sub-ambient temperatures (under such conditions, the gas phase model shown in Figure 4 should be slightly adapted to liquid phase, although the trend is not expected to be significantly affected). When moving to a higher alcohol, i.e. ethanol, the reaction is more endergonic (equation 6). The data plotted in Figure 4 reveal that, although not as favourable as for methanol, the reforming of ethanol into $\mathrm{H}_{2}$ and $\mathrm{CO}_{2}$ can be highly efficient at elevated temperatures. However, the reaction network under thermal conditions $\left(T=400-800^{\circ} \mathrm{C}\right)$ is complex, comprising a number of possible transformations which seriously affect selectivity [33]. For example, partial ethanol reforming (equation 7), water-gas shift (equation 4), ethanol dehydrogenation (equation 8), acetaldehyde decomposition (equation 9), methanation (equations 10 and 11, the latter being identical to reverse methane reforming), and several carbon/coke formation reactions, have been suggested as relevant reaction pathways. Some of these reactions, especially methanation, are more thermodynamically favourable than reforming. This is reflected experimentally in the formation of by-products, such as acetaldehyde, methane, coke, and significant amounts of $\mathrm{CO}$, thus reducing the theoretical maximum amount of $\mathrm{H}_{2}$ that can be produced [33a, 34]. Both thermodynamic models [35] and experimental data [34a, 34c] suggest that selectivities towards $\mathrm{H}_{2}$ can be enhanced at elevated temperatures and high water/ethanol ratios in the feed [35b].

$$
\mathrm{CH}_{3} \mathrm{CH}_{2} \mathrm{OH}(\mathrm{l})+3 \mathrm{H}_{2} \mathrm{O}_{(\mathrm{l})} \rightarrow 6 \mathrm{H}_{2(\mathrm{~g})}+2 \mathrm{CO}_{2(\mathrm{~g})} \quad \triangle G^{0}=97.4 \mathrm{~kJ} \mathrm{~mol}^{-1}
$$




$$
\begin{array}{ll}
\mathrm{CH}_{3} \mathrm{CH}_{2} \mathrm{OH}_{(\mathrm{l})}+\mathrm{H}_{2} \mathrm{O}(\mathrm{l}) \rightarrow 4 \mathrm{H}_{2}(\mathrm{~g})+2 \mathrm{CO}_{(\mathrm{g})} & \triangle G^{0}=137.6 \mathrm{~kJ} \mathrm{~mol}^{-1} \\
\mathrm{CH}_{3} \mathrm{CH}_{2} \mathrm{OH}_{(\mathrm{l})} \rightarrow \mathrm{H}_{2(\mathrm{~g})}+\mathrm{CH}_{3} \mathrm{CHO}_{(\mathrm{g})} & \triangle G^{0}=41.5 \mathrm{~kJ} \mathrm{~mol}^{-1} \\
\mathrm{CH}_{3} \mathrm{CHO}_{(\mathrm{g})} \rightarrow \mathrm{CH}_{4(\mathrm{~g})}+\mathrm{CO}_{(\mathrm{g})} & \triangle G^{0}=-54.7 \mathrm{~kJ} \mathrm{~mol}^{-1} \\
\mathrm{CO}_{(\mathrm{g})}+3 \mathrm{H}_{2(\mathrm{~g})} \leftrightarrows \mathrm{CH}_{4(\mathrm{~g})}+\mathrm{H}_{2} \mathrm{O}_{(\mathrm{l})} & \triangle G^{0}=-150.8 \mathrm{~kJ} \mathrm{~mol}^{-1} \\
\mathrm{CO}_{(\mathrm{g})}+4 \mathrm{H}_{2(\mathrm{~g})} \leftrightarrows \mathrm{CH}_{(\mathrm{g})}+2 \mathrm{H}_{2} \mathrm{O}_{(\mathrm{l})} & \triangle G^{0}=-130.7 \mathrm{~kJ} \mathrm{~mol}^{-1}
\end{array}
$$

If one takes acetic acid and glucose as further case studies, the competition between reforming (equations 12 and 14) and side reactions, such as decarboxylation (equation 13) or dehydration (equation 15), can also be considered.

$$
\begin{aligned}
& \mathrm{CH}_{3} \mathrm{CO}_{2} \mathrm{H}_{(\mathrm{l})}+2 \mathrm{H}_{2} \mathrm{O}_{(\mathrm{l})} \rightarrow 4 \mathrm{H}_{2(\mathrm{~g})}+2 \mathrm{CO}_{2(\mathrm{~g})} \quad \triangle G^{0}=75.4 \mathrm{~kJ} \mathrm{~mol}^{-1} \\
& \mathrm{CH}_{3} \mathrm{CO}_{2} \mathrm{H}_{(\mathrm{l})} \rightarrow \mathrm{CH}_{4}(\mathrm{~g})+\mathrm{CO}_{2}(\mathrm{~g}) \quad \triangle G^{0}=-55.3 \mathrm{~kJ} \mathrm{~mol}^{-1} \\
& \mathrm{C}_{6} \mathrm{H}_{12} \mathrm{O}_{6(\mathrm{~s})}+6 \mathrm{H}_{2} \mathrm{O}_{(\mathrm{l})} \rightarrow 12 \mathrm{H}_{2(\mathrm{~g})}+6 \mathrm{CO}_{2(\mathrm{~g})} \quad \triangle G^{0}=-84.7 \mathrm{~kJ} \mathrm{~mol}^{-1} \\
& \mathrm{C}_{6} \mathrm{H}_{12} \mathrm{O}_{6}(\mathrm{~s}) \rightarrow 6 \mathrm{C}_{(\mathrm{s})}+6 \mathrm{H}_{2} \mathrm{O}_{(\mathrm{l})} \quad \triangle G^{0}=-512.8 \mathrm{~kJ} \mathrm{~mol}^{-1}
\end{aligned}
$$

It can be clearly noticed that for these model oxygenates, reforming processes are always less energetically favourable than other possible reactions. For example, the reforming of liquid acetic acid consumes energy under standard conditions $\left(\triangle G^{0}=75.4 \mathrm{~kJ} \mathrm{~mol}^{-1}\right)$, whereas decarboxylation leading to methane and carbon dioxide is a thermodynamically spontaneous transformation $\left(\triangle G^{0}=\right.$ $\left.-55.3 \mathrm{~kJ} \mathrm{~mol}^{-1}\right)$.

In contrast to thermocatalytic processes, which suffer from limited selectivity, it has been proved by several authors that reforming of oxygenates can take place to completion (quantitative conversion to $\mathrm{H}_{2}$ and $\mathrm{CO}_{2}$, as in equations 2, 6, 12 and 14) by photocatalysis at ambient temperatures. Kawai and Sakata demonstrated in their pioneering work that sugar (sucrose) could be converted to the maximum amount of $\mathrm{H}_{2}$ and $\mathrm{CO}_{2}$ and in the expected stoichiometry (2:1) according to the ideal reforming reaction (equivalent to equation 14) in an aqueous suspension of a $\mathrm{RuO}_{2}-\mathrm{TiO}_{2}-\mathrm{Pt}$ catalyst under irradiation from a Xe lamp [36]. They also proved that complete acetic acid reforming (equation 12) could also take place on $\mathrm{Pt} / \mathrm{TiO}_{2}$ photocatalysts under $\mathrm{Hg}$ lamp light [37]. More recent work has extended the validity of this approach to other biomass-derived oxygenates such as methanol [9, 38], ethanol [9, 38a], acetic acid [38a], glycerol [9, 24], and several saccharides [9]. Yasuda and co-workers have rationalised this by extrapolating similar results to infinite dilution, whereby the theoretical maximum of $\mathrm{H}_{2}$ (and $\mathrm{CO}_{2}$ ) per mol of substrate would be obtained [39]. These observations prove that reforming of a number of oxygenated substrates can be efficiently 
and selectively carried out by means of photocatalysis. These transformations are approximately neutral regarding free energies under standard conditions (they might be slightly endergonic, whereas the sign of the energy balance can change depending on the conditions) [7d], and therefore, the fact that they take place by photocatalysis instead of other more thermodynamically favourable processes (see for example, equations 12 and 13) is exceptional and proves the selectivity of the photoreforming process.

\subsection{Energy balances and heats of combustion}

The possibility of sunlight-induced photoreforming has important implications, since part of the energy from the incident light can be stored in the form of chemical energy in the hydrogen gas produced. This is graphically represented for ethanol as a model oxygenated substrate in Figure 5. It can be clearly seen there that the energy content of the reforming product $\left(\mathrm{H}_{2}\right)$ is higher than that of the starting ethanol. From the point of view of fuel calorific power, the amount of heat which can be obtained from the generated hydrogen gas is also substantially higher than that of the oxygenated substrate. For example, the heat of combustion of one mole of the ethanol is $1366.8 \mathrm{~kJ}$ [40], whereas that of the equivalent amount of $\mathrm{H}_{2}(6 \mathrm{~mol}$, see equation 6$)$ is $1714.8 \mathrm{~kJ}$, which represents a $25.5 \%$ increase as compared to the heat content of the original substrate. This occurs for most biomass-derived oxygenated substances, as detailed in Figure 6. The most prominent increase is found for methanol $(+57 \%)$, whereas for the rest of molecules (including polyols, aldehydes, acids and saccharides), the heating value increases are all above $20 \%$. This shows that the reforming reaction of a wide range of biomass-derived substances can be noticeably favourable in terms of fuel values, as has been claimed elsewhere for actual herbaceous lignocelluloses [39c]. In the case of the photoreforming process, whereby the energy input is provided by a virtually free and inextinguishable source, that is, the Sun, the global energy balance of the process can be clearly positive since part of the energy provided by sunlight, as in the case of other up-hill photocatalytic processes such as water splitting or natural photosynthesis.

The photocatalytic reforming of oxygenates (Figure 1 and equation 1) can be viewed as the sum of the oxidation of organic substances and water splitting (equations 16 and 17, respectively).

$$
\begin{array}{ll}
\mathrm{C}_{x} \mathrm{H}_{y} \mathrm{O}_{z}+(x+y / 4-z / 2) \mathrm{O}_{2} \rightarrow y / 2 \mathrm{H}_{2} \mathrm{O}+x \mathrm{CO}_{2} & \left(\triangle G^{0}<0 ; \triangle H^{0}<0\right) \\
\mathrm{H}_{2} \mathrm{O} \rightarrow \mathrm{H}_{2}+1 / 2 \mathrm{O}_{2} & \triangle G^{0}=237.1 \mathrm{~kJ} \mathrm{~mol}^{-1}
\end{array}
$$

The oxidation of organic substances is exergonic and exothermic, whilst water splitting is an energy-consuming process. As discussed previously, the combination of both reactions may result in slightly up-hill reforming processes. 


\subsection{Efficiency of radiative energy use}

Effectively, in the reforming reaction water acts as an oxidising agent, in an analogous manner to molecular oxygen in combustion. In the former case, $\mathrm{H}_{2}$ is released by reduction of $\mathrm{H}_{2} \mathrm{O}$, whereas in the latter, $\mathrm{O}_{2}$ is reduced to $\mathrm{H}_{2} \mathrm{O}$. In the particular context of photocatalysis, this can be graphically represented as shown in Figure 1. The separated charges (electrons and holes) generated in the photocatalyst upon absorption of light trigger the reactions which lead to the reforming of the biomass-derived substances in the presence of water (reduction of water and oxidation of the substrate, respectively).

Photocatalytic reactions are generally initiated upon light absorption by a semiconductor. This phenomenon occurs when the wavelength of the incident light corresponds to an amount of energy equal to or greater than that of the semiconductor band gap, that is, the energy difference between the top of its valence band and the bottom of its conduction band. Further, each of the half-reactions involved will proceed only if the following requirements are met: $(i)$ the potential of the conduction band is more negative (i.e. its energy is higher) than that of the reduction half-reaction, and (ii) the potential of the valence band is more positive (i.e. its energy is lower) than that of the oxidation half-reaction. In that case, electrons may be transferred $(i)$ from the conduction band into the species to be reduced, and (ii) from the species to be oxidised into the valence band. After these processes are completed, the semiconductor returns to its ground state and the photocatalytic cycle can continue [5d, 7e, 23]. For endergonic reactions, such as natural photosynthesis or the reforming of oxygenates, light energy is stored in this way. The reduction of protons to molecular hydrogen is theoretically feasible on a number of excited semiconductors in the presence of appropriate cocatalysts [5d, 7e, 41]. For example, the energy level of the conduction band of $\mathrm{TiO}_{2}\left(E_{\mathrm{CB}}=\right.$ $-4.21 \mathrm{eV})$ is higher than the $\mathrm{H}^{+} / \mathrm{H}_{2}$ reduction potential $(E=-4.5 \mathrm{eV}$ with respect to the vacuum level or, by convention, $E^{0}=0 \mathrm{~V} v s$. the normal hydrogen electrode, NHE). On the other hand, the energy level of the edge of the valence bands of many semiconductors is expected to be lower than the oxidation potentials for most organic substances, a fact which is responsible for the high efficiencies of the photocatalytic mineralisation of organic pollutants (to $\mathrm{CO}_{2}$ ) with concomitant reduction of $\mathrm{O}_{2}[23,42]$, and for the same reason, should be equally efficient for photoreforming since the oxidation half-reactions are formally the same (see Figure 1) [7d]. Thus, once the energy barrier for the process has been overcome by virtue of the absorbed light, the efficiency of the process will be dictated by the ability of the photocatalyst to provide accessible active sites. In short, that ability can be referred to as photocatalytic activity, and can be measured by means of the 
quantum yield $(\Phi)$, defined by equation 18. Since the amount of absorbed photons is extremely difficult to be measured, in practice apparent quantum yields are used $\left(\Phi_{\text {ap }}\right)$, defined by equation 19 .

$\Phi=100 \times \frac{n \text { (transferred electrons })}{n(\text { absorbed photons })}$

$\Phi_{\mathrm{a}}=100 \times \frac{n \text { (transferred electrons })}{n \text { (incident photons })}$

Then, charges generated upon light absorption should migrate to the active sites and participate in reactions. Otherwise, electrons and holes may recombine and thus release energy, causing reductions in quantum yields.

\section{Mechanism}

Surface species responsible for charge transfer reactions, that is, active sites of the solid catalyst and adsorbates from the substrate, are the subject of mechanistic studies on photocatalytic processes, although the knowledge of their identity is not a trivial issue. Spectroscopic techniques can provide useful information for that purpose. However, in situ operando studies, which are obviously most suited to elucidate reaction mechanisms, are often not feasible in the course of the photocatalytic experiments. As an approximation to the real situations, some investigations are devoted to similar measurements dealing with surface phenomena in the dark. In this section, we will summarise the most relevant information about the mechanisms of oxygenate photoreforming, highlighting those hypotheses which have raised more consensus. Kinetic studies will be also the focus here.

\subsection{Photo-generation of charges upon Light absorption}

It is widely accepted that semiconductors are active materials for the absorption of light and that a number of chemical processes can be unleashed upon such phenomenon. This can be experimentally proven by comparing the photocatalytic activities of materials based on semiconductors (e.g. $\mathrm{TiO}_{2}$ ) to those of expectedly non-active isolating analogues (e.g. $\left.\mathrm{SiO}_{2}\right)$ [43], although it should be noted that the latter may also activate photolytic events under intense ultraviolet-visible (UV-vis) light [44]. As mentioned above, photo-generated charges should migrate to the active sites before recombination. The properties, behaviour and performance of titanium dioxide in this context has been extensively investigated and is thus well understood for many applications [6a], whereas analogous knowledge about other semiconductors is more scarce.

4.1.1. Time-resolved spectroscopic evidence for transient photo-generated electrons. The observation of charge separation events on semiconductor particles is a challenging task. The best 
approach to date relies on time-resolved infrared (IR) absorption spectroscopy of laser-irradiated samples [6a, 6b], a method designed in an elegant experimental set-up by Onishi, Ishitani and coworkers [45]. It consists of a laser source emitting UV or visible pulses which travel through a sample. After irradiation, light from an IR source is focused on the sample. An AC-coupled amplification of the transmitted IR signal allows detection of small transient absorbance changes with a time resolution of $50 \mathrm{~ns}$. A characteristic feature-less (no bands) absorption, observed in systems containing $\mathrm{TiO}_{2}$, was assigned to photo-generated electrons trapped in shallow mid-gap states, which may be promoted to higher-energy conduction band states (see Figure 7). According to the authors, absorption by photo-generated holes in the IR frequency range is ruled out due to the theoretically wide energy difference between mid-gap trap states and the top of the valence band [45]. In the case of titania materials under vacuum, the transient IR signals decrease along a multiexponential rate law with time, a fact which is related to the recombination of photo-generated electrons and holes [45a]. The decay kinetics of the electron is sensitive to different atmospheres. For example, $\mathrm{O}_{2}$ accelerates the decay due to its tendency to consume electrons (Figure 8, left). In contrast, $\mathrm{H}_{2} \mathrm{O}$ consumes holes and therefore causes an accumulation of electrons, which reduce Ti(IV) to Ti(III) and results in a slower decay of the transient electron signal (Figure 8, right) [46]. This reduction was inferred by a colour change of the material from white to blue [46-47], and may result in the formation of oxygen defects or even $\mathrm{Ti}_{2} \mathrm{O}_{3}$ phases [47a]. Similar observations have been described in the presence of oxygenated substances - prone to undergo oxidation - such as 2propanol [48] or glycerol, as far back as in 1921 [49]. Irradiation of titania materials bearing supported platinum nanoparticles $\left(\mathrm{Pt} / \mathrm{TiO}_{2}\right)$ under vacuum results in faster decays, a fact which is ascribed to electron migration to Pt after times as short as $1 \mu$ s (Figure 9, left) [46]. In the presence of $\mathrm{H}_{2} \mathrm{O}$ vapour, similar hole consumption is observed for $\mathrm{TiO}_{2}$ before $2 \mu \mathrm{s}$, whereas the decay is significantly accelerated during $10^{1}-10^{3} \mu$ s time spans for $\mathrm{Pt} / \mathrm{TiO}_{2}$ (Figure 9, centre). This is due to reduction of $\mathrm{H}_{2} \mathrm{O}$ to $\mathrm{H}_{2}$ by electrons at the surface of $\mathrm{Pt}$ nanoparticles at longer times [46, 50]. The same authors performed similar experiments in the presence of methanol [51] or methanol/water [52] vapours. Methanol suppressed such decay, indicating a more efficient hole consumption than in the case of water [51-52]. Adding $\mathrm{H}_{2} \mathrm{O}$ to the system inhibited methanol oxidation by holes to some extent, although this was followed by faster electron consumption at longer times, most likely related to the evolution of hydrogen by reduction of the thereby more ubiquitously available protons [52]. Similar results were reported for 2-propanol on $\mathrm{Pt} / \mathrm{TiO}_{2}$ [53]. On the other hand, materials based on La-doped $\mathrm{NaTaO}_{3}$ having supported $\mathrm{NiO}$ particles exhibited similar photoresponse, proving that electron transfer onto nickel and subsequent reduction of water to $\mathrm{H}_{2}$ on such metal (thus acting as a co-catalyst) can be also promoted [54]. 


\subsubsection{Time-resolved spectroscopic evidence for transient photo-generated holes. A}

complementary method to transient electron studies, developed by Shkrob and Sauer, revealed that photo-generated holes in $\mathrm{TiO}_{2}$ absorb at visible and near-IR wavelengths. They suggested the formation of two types of holes depending on whether these were located at surface sites or in deeply trapped states, and their distinct decay kinetics: faster for the former and slower for the latter, due to oxidation of either chemisorbed or physisorbed glycerol, respectively [55]. The direct detection of photo-generated holes was also reported by Furube and co-workers [56]. This was achieved at femtosecond to nanosecond time scales by UV-vis absorption after low intensity excitation to avoid fast electron-hole recombination. Trapped holes in nanocrystalline $\mathrm{TiO}_{2}$ films display a characteristic absorption maximum at $500 \mathrm{~nm}$; alcohols caused significant decreases of the signal, thus confirming their strong tendency to react with holes and thus suppress recombination (Figure 10). An important point to note here is that, in the presence of alcohols, the lifetimes of photo-generated holes is significantly shorter $\left(10^{-12}-10^{-9} \mathrm{~s}\right)$ than those of photo-generated electrons $\left(10^{-5}-10^{-3} \mathrm{~s}\right)$. Therefore, substrate oxidation events in photoreforming are expected to be significantly faster than proton reduction to generate $\mathrm{H}_{2}$ by the complementary half-reactions.

\subsection{Adsorption-dependent kinetics}

4.2.1. Postulation of adsorption-dependent models. For photocatalytic reforming of oxygenated organic molecules in aqueous suspensions of semiconductor powders, it has been demonstrated that reaction rates commonly increase with oxygenated substrate concentration. The increase is sharp at low concentrations, but rates asymptotically reach a maximum at higher concentrations, indicating a near-zero order dependence with respect to the substrate. This is consistent with a Langmuir-type model, whereby rates are directly dependent on the amounts of organic species adsorbed, and no further increase takes place above saturation of the photocatalyst surface. The rates of $\mathrm{H}_{2}$ production were observed to increase in this way with methanol concentration on aqueous suspensions of $\mathrm{Pd} / \mathrm{TiO}_{2}$ powders, reaching a near-zero order dependence [57]. Similar behaviours were reported for methanol [38c, 58], 2- propanol [59], oxalic acid [60], acetic acid [61], glucose [62] or sucrose [62b] on $\mathrm{Pt} / \mathrm{TiO}_{2}$, for glucose [63] or glycerol [64] on $\mathrm{Cd}_{x} \mathrm{Zn}_{1-x} \mathrm{~S}$, or for glucose on $\mathrm{ZnS}-\mathrm{ZnIn}_{2} \mathrm{~S}_{4}$ [58] photocatalysts. In some of these reports, in particular dealing with glucose photoreforming, data were successfully fitted to the Langmuir-Hinshelwood kinetic model $[58,62 \mathrm{a}]$, expressed as:

$\frac{1}{r\left(\mathrm{H}_{2}\right)}=\frac{1}{k\left(\mathrm{H}_{2}\right)}+\left(\frac{1}{k\left(\mathrm{H}_{2}\right) K}\right) \frac{1}{C_{0}}$

where $r\left(\mathrm{H}_{2}\right), k\left(\mathrm{H}_{2}\right), K$ and $C_{0}$ are the initial $\mathrm{H}_{2}$ production rate, the rate constant of $\mathrm{H}_{2}$ evolution, the adsorption constant of glucose and the initial concentration of glucose, respectively. A graphical 
representation of this substrate adsorption-based relationship is shown in Figure 11. In another particular case, the adsorption-rate relationship was fitted to the square root of methanol concentration, and this was ascribed to a dissociative adsorption model [58]. Linear dependences of $\mathrm{H}_{2}$ production rates and the logarithms of glycerol, ethanol or galactose concentrations were alternatively observed, albeit no explanation was stated for such model [9].

4.2.2. Suitability and utility of kinetic models. The interpretations stated above should be considered with care, since the Langmuir-Hinshelwood model represents a one-step reaction of adsorbates, whereas the photoreforming of oxygenated molecules involve multiple reaction steps. The graphical representations which relate the maximum $\mathrm{H}_{2}$ production rates to the initial concentration of the substrate should thus contemplate this fact. In an essay article, Ohtani stressed that postulation of a Langmuir-Hinshelwood model requires the measurement of adsorption data in the dark for validation [42]. However, based on the first order rate constants measured at low concentrations and zero-order at higher concentrations, it can be derived that organic substrate oxidation proceeds efficiently and rapidly on the surface of the photocatalyst, so that the process is only limited by diffusion and adsorption kinetics and not by the rate of surface reaction under adsorption equilibrium [6c, 42]. In practice, this is beneficial since charge recombination is prevented by fast charge transfer at the catalyst surface-solution interphase. Consequently, electrons can be also efficiently transferred to protons by appropriate co-catalysts, giving rise to the formation of hydrogen gas. This is the basis of the photocatalytic production of $\mathrm{H}_{2}$ from water aided by alcohols, which are considered as electron donors for the process. In fact, in the absence of alcohols or other sacrificial electron donors, pure water splitting is not efficient on most $\mathrm{M} / \mathrm{TiO}_{2}(\mathrm{M}=$ noble metal) materials since they are not active for the oxidation of water to $\mathrm{O}_{2}$ [6c]. However, they can catalyse the oxidation of organic substances at high rates. This was clearly illustrated by Kondarides et al., who showed that the quantum yields for $\mathrm{H}_{2}$ production were noticeably higher for an aqueous glycerol solution $(1 \mathrm{M})$ than for pure water $(\Phi>70 \%$ and $\Phi=1.8 \%$ at maximum rate, respectively) on the same kind of $\mathrm{Pt} / \mathrm{TiO}_{2}$ photocatalysts [9]. In this line, a time-resolved IR spectroscopic study related the efficiency of methanol reforming to its rapid oxidation into methoxide radicals and then, to the slow recombination of electrons with such oxidised species, in contrast to the more favoured direct recombination with holes or secondary recombination with hydroxide radicals in pure water [65].

\subsection{Oxidation mechanisms}

4.3.1. Comparison of aerobic photo-oxidation and anaerobic photoreforming. As described in the previous section and sketched in Figure 1, the photocatalytic reforming of oxygenated 
substances can be considered as the sum of proton reduction into molecular hydrogen and oxidation of the carbon feedstock into $\mathrm{CO}_{2}$. The oxidation processes are mainly promoted by the generation of holes in the valence band of the semiconductor. In the presence of $\mathrm{O}_{2}$, hydroxyl radicals may be originated by oxidation of water or surface hydroxide anions by holes, or by the reduction of molecular oxygen. In addition to hydroxyl radicals, other activated oxygen species such as peroxides $\left(\right.$ e.g. $\left.\mathrm{H}_{2} \mathrm{O}_{2}\right)$ or superoxide species, including the superoxide anion $\left(\mathrm{O}_{2}{ }^{--}\right)$or the hydroperoxyl radical $\left(\mathrm{HO}_{2}{ }^{\circ}\right)$ can be formed in noticeable concentrations mostly by $\mathrm{O}_{2}$ reduction, thus giving rise to a complex network of oxidation reactions, both in solution and on the surface of the catalyst [66]. Therefore, photocatalytic oxidation, although being light-initiated, might in fact proceed by propagation of free-radical processes to a significant extent, as for example evidenced by kinetic analyses [67]. Once a dioxygen molecule is first reduced to superoxide, this sequence of steps whereby active oxygen species act as direct electron acceptors can be activated without the participation of photons, but rather thermally (see Figure 12) [66a]. In contrast, the oxidation of the organic substrate in photocatalytic reforming in the absence of $\mathrm{O}_{2}$ (where water is the ultimate oxidising agent) can only be promoted by light, either directly by holes or indirectly by hydroxyl radicals, since there is no possibility of the reaction to proceed via thermally activated free-radical pathways. These fundamental differences between photocatalytic oxidation and reforming are schematically illustrated in Figure 12.

It should be noted that peroxides may also be formed in photoreforming processes as secondary active oxygen species (Figure 12, bottom). In fact, this was proven in a thorough study dealing with the quantification of peroxides during irradiations of aqueous $\mathrm{Pt} / \mathrm{TiO}_{2}$ suspensions containing glycerol. The kinetic data provided is strongly consistent with the suggested mechanisms involving hydroxyl radicals as the primary oxidising agent and peroxides as a secondary species taking part in the oxidation processes, as shown in Figure 12. The complete reduction of an $\mathrm{O}_{2}$ molecule into $\mathrm{H}_{2} \mathrm{O}$ (involving the transfer of $4 \mathrm{e}^{-}$) in aerobic photo-oxidation can be unleashed by the absorption of a single photon, yet the participation of further photons may also be at play. Therefore, the scenario where one photon activates an oxygen molecule which in turn may initiate up to three further charge transfer events by thermal activation cannot be neglected. In contrast, each electron transfer in photoreforming can uniquely be activated by absorption of one photon, even where peroxides $\left(\mathrm{H}_{2} \mathrm{O}_{2}\right.$ and related species, see Figure 12) take part, a fact which implies that photoreforming quantum efficiency calculations can be safely based on the total amount of $\mathrm{H}_{2}$ produced.

4.3.2. Direct $v s$. indirect oxidation. In photocatalysis, the initial oxidation steps may proceed by two different routes: $(i)$ direct oxidation of chemisorbed or strongly adsorbed species by holes on 
the surface of the photocatalyst, or ( $i i)$ indirect oxidation reaction of the physisorbed organic species with previously formed hydroxyl radicals in outer adsorption spheres [6a, 23, 66a, 68]. Whatever the oxidation route, the resulting oxidation intermediates and products might be identical, and thus, discrimination between these two routes is challenging. Regarding the discrimination between direct and indirect oxidation in the photoreforming of oxygenated substrates, Kawai et al. concluded that the significance of each route for alcohols depends on the energy of their valence orbital energy positions. They concluded that alcohols with more tendency to be oxidised (e.g. ethanol) were transformed directly by holes on $\mathrm{Pt} / \mathrm{TiO}_{2}$, whereas those with energetically deeper valence orbitals are indirectly oxidised by $[\mathrm{OH}]^{\circ}$, a fact evidenced by the dependence of water content in the system on the reaction rates [69]. In contrast, kinetic studies performed on different substrates were not conclusive for discerning between either direct oxidation by holes or indirect oxidation by $[\mathrm{OH}]^{\circ}$, since activation energies were indistinguishable for the range of alcohols studied, including methanol, ethanol, 1-propanol and 2-propanol [70]. This was attributed to electron transfer between the bulk of the photocatalyst and the surface active species as the common rate-limiting step, irrespective of the alcohol substrate, which was believed to react rapidly [70-71]. Probably the most reliable and revealing evidence of the primary steps in alcohol photoreforming is that obtained from in situ transient IR absorption spectroscopy (see Section 4.1), which suggested that methanol consumes holes more rapidly in the absence of water, and increasing the amount of water vapour prevents the process accordingly [52]. These results are in favour of the direct holepromoted electron abstraction for alcohols on irradiated photocatalysts. In this line, in situ EPR studies reported by Thurnauer and co-workers revealed that the only radical produced on irradiated aqueous colloidal $\mathrm{TiO}_{2}$ from alcohols were the corresponding 1-hydroxyalkyl species, where $\alpha$ hydrogen atoms had been abstracted [72]. Neither free nor adsorbed hydroxyl radicals were detected, and instead, it was suggested that holes were trapped as surface oxidised oxygen atoms [72a]. Both facts thus indicate direct oxidation by holes of the alcohols, either as strongly adsorbed species or as loosely adsorbed molecules within a few monolayers from the photocatalyst surface. These assumptions are consistent with those stated by Howe and Grätzel and based on EPR measurements on hydrated anatase samples [73], but nevertheless opposed to the previous interpretation of similar data by Anpo et al., which proposed the existence of hydroxyl radicals in those UV-irradiated systems [74].

Nosaka et al. measured the relative amount of radicals formed by in situ electron paramagnetic resonance (EPR) spectroscopy, and proposed adsorption kinetics as another factor which may influence the primary (direct/indirect) routes of acetic acid reforming on irradiated aqueous $\mathrm{Pt} / \mathrm{TiO}_{2}$ 
[68a]. They based their argument in the observation of two distinguishable routes depending on $\mathrm{N}_{2}$ flow rate through the suspension: at low gas flow rates diffusion of acetic acid would be the ratedetermining step and its (direct) oxidation by holes would take place predominantly, whereas at high gas flow rates reaction rates are almost unaffected by diffusion and formation of hydroxyl radicals and subsequent (indirect) oxidation of acetic acid would be more significant [68a]. Another study relating the efficiency of $\mathrm{H}_{2}$ production from different oxygenated substrates on illuminated $\mathrm{Pt} / \mathrm{TiO}_{2}$ to the rate of hydrogen abstraction in solution led to conclude that adsorbed hydroxyl radicals might be the oxidising agents in this step [75]. A recent study by Bahnemann and coworkers is also consistent with this conclusion [76]. Li et al. observed the formation of [OH] ${ }^{\bullet}$ in illuminated aqueous $\mathrm{ZnS}-\mathrm{ZnIn}_{2} \mathrm{~S}_{4}$ photocatalyst suspensions, and related their occurrence with glucose photoreforming [58].

Probably the most insightful mechanistic study regarding the distinction between direct or indirect oxidation steps was that reported by Selli and co-workers on the vapour-phase photoreforming of methanol using $\mathrm{M} / \mathrm{TiO}_{2}(\mathrm{M}=\mathrm{Au}$ or $\mathrm{Pt})$ as photocatalysts [77]. They developed a thorough kinetic model (supported by isotopic exchange experiments) which satisfactorily fitted their experimental data for steady-state production of $\mathrm{HCHO}, \mathrm{HCO}_{2} \mathrm{H}$ and $\mathrm{CO}_{2}$ as a function of the molar $\mathrm{H}_{2} \mathrm{O} / \mathrm{CH}_{3} \mathrm{OH}$ ratio, and distinguished between three different mechanistic routes: $(i)$ indirect $[\mathrm{OH}]^{\circ}$-mediated, (ii) direct hole-mediated, and (iii) direct water-assisted routes. The three pathways proved to proceed in parallel, being the influence of each of them dependent on the composition of the vapour phase. Under methanol-rich regimes, direct oxidation by holes leading to $\mathrm{HCHO}$ would be the only relevant route, whereas upon increasing water content, indirect oxidation by hydroxyl radicals would be predominant. Intermediate situations could not be accounted for by the simplistic direct/indirect dichotomy, and a third route whereby the water-rich medium facilitated proton transport from oxidation to reduction sites - which then needed not be contiguous at the periphery of the metal/titania interface, and could be far apart on pure titania or metal surfaces, respectivelywas proposed. Based on this work, the possibility of several mechanistic routes at interplay is always a wise hypothesis considering complex photoreforming processes [77].

\subsection{The speciation of adsorbed substrates and intermediates}

Despite some mismatching data, the efficiency of complete photoreforming of the many different substrates is apparent from the large amount of tests reported in the literature. Spectroscopic investigations are valuable in order to gain a more insightful knowledge of the mechanism, as outlined in the following paragraphs. 
4.4.1. Speciation of adsorbed substrates. 4.4.1.1. Adsorption of alcohols. Regarding the interaction of oxygenates with titania surfaces, a limited number of spectroscopic studies have been devoted to identifying the mode of alcohol adsorption. Nosaka and co-workers measured ${ }^{1} \mathrm{H}$ NMR spectra of $\mathrm{TiO}_{2}$ in the solid state using a magic angle spinning (MAS) probe, in the presence of moisture and ethanol and at variable temperatures, concluding that ethanol is more strongly bound to the surface of titania than water, even remaining after heating at $150{ }^{\circ} \mathrm{C}$; however, the ethanol signals disappear after UV irradiation, thus suggesting rapid decomposition (Figure 13) [78]. Similar conclusions were drawn from a sum-frequency generation spectroscopic study for methanol, and two possible mechanistic pathways were proposed: in the absence of water, adsorbed methoxide would be directly oxidised by holes, whereas in the presence of water, methanol would be indirectly oxidised by photo-generated hydroxyl radicals [79]. In the gas phase, condensation of alcohols with titanol groups has been suggested based on the disappearance of (Ti)O-H stretching bands, the observation of characteristic water vibrations and the shift of certain bands of the alcohol due to chemisorption $[51-52,65,69,80]$. In this line, adsorption of ethanol as ethoxide on $\mathrm{Pt} / \mathrm{TiO}_{2}$ was observed by conventional IR methods [69]. Analogous methanol adsorption from the gas phase has also been observed [51-52, 80f]. In these cases, methoxide remained on the photocatalyst after evacuation under reduced pressure [51-52, 80f], whereas molecular methanol was readily removed in this way [52]. Adsorption of methanol on similar $\mathrm{Pt} / \mathrm{TiO}_{2}$ solids with different $\mathrm{Pt}$ contents was also studied by transmission FTIR, revealing that: (i) methoxide is preferentially formed, and (ii) the amount of adsorbed species markedly decreases with increasing Pt content (up to 1.0\%) [65]. This was explained based on the importance of surface oxygen vacancies as methoxide formation sites and, in competition, also as Pt nucleation centres, a fact which would be responsible for existence of maximum $\mathrm{H}_{2}$ production at relatively low $\mathrm{Pt}$ loadings.

4.4.1.2. Adsorption of carboxylic acids. The interaction of carboxylic acids on titania materials and their subsequent photodecomposition has also been studied by means of FTIR spectroscopy. Attenuated total reflectance (ATR) has served to elucidate how several substrates adsorb on titania surfaces. Formic or oxalic acid sorption on $\mathrm{TiO}_{2}$ was studied by ATR-IR spectroscopy on samples prepared from aqueous suspensions after water evaporation (Figure 14, top) [81]. The absence of $v(\mathrm{C}=\mathrm{O})$ bands suggested that formic acid deprotonated into adsorbed formate species. Furthermore, oxalic acid adsorbed more strongly and preferentially via bidentate bonding, and thus, its photodecomposition (into $\mathrm{H}_{2}$ ) was faster even in the presence of a large formic acid excess. This proves the importance of substrate adsorption on $\mathrm{H}_{2}$ production by photocatalytic reforming. Glyoxylic [82] and lactic acid [83] adsorbed from the solution onto $\mathrm{TiO}_{2}$ in a bidentate chelating fashion; in contrast lactic acid interacted electrostatically as lactate anion with CdS surfaces (Figure 
14, bottom) [83]. The identification of such adsorption modes was used to account for the different products formed from reactions on the corresponding illuminated platinised photocatalysts: dehydrogenation was observed on $\mathrm{Pt} / \mathrm{CdS}$, whilst oxidative decarboxylation took place on $\mathrm{Pt} / \mathrm{TiO}_{2}$, possibly favoured by the strongest, more intimate, chelating interaction involved [84].

4.4.2. In situ spectroscopic monitoring under irradiation. 4.4.2.1. Evolution of adsorbed alcohols under irradiation. Regarding the effect of light on adsorbed alcohols, direct observation by EPR allowed to identify hydroxymethyl or 1-hydroxyethyl as the primary products from the interaction of adsorbed methanol [72] or ethanol [72b] with illuminated colloidal $\mathrm{TiO}_{2}$. The primary alcohol oxidation steps on $\mathrm{M} / \mathrm{TiO}_{2}(\mathrm{M}=\mathrm{Pt}, \mathrm{Pd}, \mathrm{Rh}, \mathrm{Ir}, \mathrm{Ru}$ or $\mathrm{Os})$ were also successfully monitored in detail by using in situ EPR spectroscopy in aqueous suspensions. Thus, hydroxymethyl, 1hydroxy-1-ethyl and 2-hydroxy-2-propyl radicals were detected from methanol, ethanol and 2propanol, respectively [85]. These independently reported results unambiguously revealed the dehydrogenation in $\alpha$ positions with respect to the hydroxyl group, although the formation of transient (undetected) hydroxyl radicals, that is, where the unpaired electron is located on the oxygen atom cannot be ruled out [65].

The strong adsorption of alcohols on titania and their subsequent degradation upon UV light irradiation have also been observed by means of FTIR spectroscopy. Time-resolved IR absorption suggested the rapid (<50 $\mu$ s) dehydrogenation of 2-propanol into acetone on $\mathrm{Pt} / \mathrm{TiO}_{2}$ irradiated with a $355 \mathrm{~nm}$ laser pulse [53]. Similarly, the use of a specially designed cell allowed the measurement of FTIR spectra after UV light irradiation of $\mathrm{Pt} / \mathrm{TiO}_{2}$ samples with adsorbed 2propanol, leading to the detection of acetone (primary product), adsorbed carboxylates (acetate and formate), $\mathrm{CH}_{4}$ and $\mathrm{CO}_{2}$, as degradation products [80a]. A mechanism based on dehydrogenation (first step), C-C scission and decarboxylation reactions was proposed. In the complete study by $\mathrm{Li}$ on methanol photoreforming, several products adsorbed on the solid-gas interface, such as formaldehyde, dioxymethylene, formate and formic acid were detected by FTIR after UV irradiation from a laser source [65]. A similar approach was also used to monitor the methanol reforming reaction on illuminated $\mathrm{Pt} / \mathrm{TiO}_{2}$; formate and carbonate species were detected as products or intermediates [80f]. Moreover, ex situ diffuse reflectance infrared Fourier transform spectroscopy (DRIFTS) also allowed the detection of formaldehyde, dioxymethylene and formic acid as methanol photoreforming products on $\mathrm{Pt} / \mathrm{TiO}_{2}$ photocatalysts after reactions in UV-irradiated suspensions [38c]. Interestingly, these intermediates were adsorbed on the solid, and not dissolved in the liquid phase. 
4.4.2.2. Evolution of adsorbed carboxylic acids under irradiation. McQuillan and co-workers specially designed cells for the measurement of in situ ATR-IR spectra of solid photocatalyst films in contact with aqueous solutions, which allow the detection of adsorbates and the monitoring of their evolution under light irradiation; this approach was successful for the identification of chelating glyoxylate transformation into oxalate by oxidation of the aldehyde moiety on $\mathrm{TiO}_{2}$ under UV light, based on the disappearance and appearance of the corresponding $\mathrm{C}=\mathrm{O}$ stretching bands [82]. Gas phase FTIR studies of formic acid $\mathrm{Pt} / \mathrm{TiO}_{2}$ have been also performed on pressed disc samples, leading to the conclusion that both protonated and deprotonated forms can exist adsorbed on the catalyst [86]. Upon UV (355 nm) laser irradiation, the former (molecular species) is degraded more rapidly than the latter (anionic species), although both are transformed into carbonates, eventually leading to $\mathrm{CO}_{2}$ and $\mathrm{H}_{2}$. Anionic formate adsorbed strongly on bare $\mathrm{TiO}_{2}$ surfaces, remaining even after evacuation at high temperatures, as suggested by FTIR, whereas $\mathrm{CO}_{2}$ evolved readily due to formate decomposition under UV-vis light [80g].

A more sophisticated set-up based on a surface enhanced infrared absorption (SEIRA) technique was developed by Sato and co-workers to track the UV-induced degradation of acetic acid on $\mathrm{Au} / \mathrm{TiO}_{2}$ or $\mathrm{Au} / \mathrm{Pt} / \mathrm{TiO}_{2}$ photocatalyst layers. Adsorption of molecular acetic acid was observed in gas phase experiments, whereas both acid and carboxylate species formed on the surfaces of the materials in liquid aqueous phases. Upon irradiation, carbonate species were detected on $\mathrm{Au} / \mathrm{TiO} 2$ films, whereas in addition, methyl radicals were also found on analogous $\mathrm{Au} / \mathrm{Pt} / \mathrm{TiO}_{2}$ [87]. The formation of methyl radicals from irradiated acetic acid samples had also been previously confirmed by EPR on $\mathrm{Pt} / \mathrm{TiO}_{2}$ in the liquid phase [68a, 85a, 88]. The mentioned intermediates (carbonates and methyl radicals) support a mechanistic pathway involving decarboxylation of acetic acid and accounting for the eventual formation of $\mathrm{CH}_{4}$ and smaller amounts of $\mathrm{C}_{2} \mathrm{H}_{6}$ by coupling of methyl radicals with adsorbed hydrogen atoms $\left(\mathrm{H}^{*}\right)$ or with themselves, respectively. Furthermore, reduction of protons would lead to the formation of $\mathrm{H}_{2}$ [88a, 89]. If a maximum production of $\mathrm{H}_{2}$ is sought, reforming mechanisms involving the complete oxidation of carbon-containing intermediates should be investigated. This is especially important for alkyl radicals (e.g. methyl) generated by decarboxylation, which tend to couple with other alkyl or hydrogen radicals, resulting in the formation of alkanes (e.g. methane) and thus, in a decrease of $\mathrm{H}_{2}$ selectivities.

\subsection{Postulation of different mechanistic routes}

In addition to investigations on surface species, some studies have focused on tracking reaction intermediates in solution. Combining the knowledge from both approaches, the elucidation of the most plausible photoreforming mechanisms has been attempted by several researchers. 
4.5.1. Alcohol photoreforming mechanisms. The significance of different alcohol reforming mechanisms on $\mathrm{M} / \mathrm{TiO}_{2}(\mathrm{M}=$ noble metal) has been investigated by independent research groups on the basis of the product analyses (by mass spectrometry and $\mathrm{H}_{2}$ quantification) for alcohols and other oxygenates having slightly different structures [90]. They observed that dehydrogenation is generally efficient provided $\alpha$-hydrogens are available, as generally observed in a number of other studies. In a further mechanistic step, the presence of terminal alkyl groups would lead to the production of the corresponding alkane (for example, methane from the methyl group in ethanol). In polyols without terminal alkyl groups no alkane-type products could be detected. Instead, $\mathrm{H}_{2}$ and $\mathrm{CO}_{2}$ were the main products in molecules with several hydroxyl groups [90b]. In order to account for the aforementioned product distribution, different mechanisms have been postulated for hydroxyl-containing molecules, as detailed in the following sections.

4.5.1.1. The CO mechanistic route. This mechanism involves the non-selective dissociation of the adsorbed alkoxides into $\mathrm{CO}$ and atomic hydrogen, both adsorbed on noble metal particles, and alkanes (referred to here as the CO route, see Figure 15). This hypothesis is based on temperature programmed desorption (TPD) experiments, and further oxidation of $\mathrm{CO}$ into $\mathrm{CO}_{2}$ [26, 57, 90a, 91] The latter step might follow a water gas shift stoichiometry (water as the oxidising agent), a process which takes place efficiently on $\mathrm{Au} / \mathrm{TiO}_{2}$ under simulated solar light [92], or on $\mathrm{Pd} / \mathrm{TiO}_{2}$ irradiated by a Xe lamp [93]. Complementarily to the CO route mechanism for primary alcohols (Figure 15), Bowker and co-authors also suggested that dehydrogenation of secondary counterparts may proceed by beta-hydride elimination and formation of a carbonyl group and then, subsequent decarbonylation of the ketone would result in $\mathrm{CO}$ and two alkyl radicals [90a], via a reaction which involves two $\mathrm{C}-\mathrm{C}$ bond scissions. It should be reminded that this mechanism, happening via intermediate $\mathrm{CO}$, is based on high temperature data (TPD), and thus, on substantially different experimental conditions than those used in near-ambient temperature photocatalysis. In support of the route suggested by Bowker and co-workers, small amounts of $\mathrm{CO}$ have been quantified by photoreforming of methanol [94], glycerol [94b] or glucose [94b, 95] on $\mathrm{M} / \mathrm{TiO}_{2}(\mathrm{M}=\mathrm{Ni}, \mathrm{Cu}, \mathrm{Pt}$, $\mathrm{Rh}, \mathrm{Ru}, \mathrm{Au}$ or Ir) photocatalysts.

4.5.1.2. The carbonyl mechanistic route. A different mechanism was postulated by $\mathrm{Fu}$ et al. for a range of similar substrates on $\mathrm{Pt} / \mathrm{TiO}_{2}$ photocatalysts (referred to here as the carbonyl route, see Figure 16) to account for the formation of carbonyl moieties [96]. According to this hypothesis, carbonyl-containing primary products of alcohol dehydration undergo oxidative C-C cleavage giving rise to an alkyl radical and a carboxylic acid [62a, 96]. Direct evidence for the formation of 1-hydroxyalkyl-type radicals, derived from primary alcohol oxidation by abstraction of $\alpha$-hydrogen 
atoms, was proven by direct observation using EPR spectroscopy for methanol, ethanol or 2propanol on $\mathrm{M} / \mathrm{TiO}_{2}(\mathrm{M}=\mathrm{Pt}, \mathrm{Pd}, \mathrm{Rh}, \mathrm{Ir}, \mathrm{Ru}$ or $\mathrm{Os})$ suspensions [85a]. Another proof to the hydrogen abstraction route is the coupling of the derived radicals into dimers whereby new $\mathrm{C}-\mathrm{C}$ bonds are formed [75, 97]. On most occasions, dehydrogenation is the dominant mechanistic pathway after the initial hydrogen abstraction, as evidenced by the large number of studies reporting the formation of carbonyl functionalities from the corresponding alcohols.

Moreover, this mechanism contemplates the oxidation of alkyl radicals formed by C-C scission into smaller alcohols, which can in turn adsorb onto titania and undergo a further reforming cycle, instead of undergoing reductive coupling into alkanes. This route would account for the favoured formation of $\mathrm{H}_{2}$ from polyols such as glycerol, and to the disfavoured formation of alkanes such as methane [96]. In other words, it can explain the full reforming of the starting oxygenate into $\mathrm{H}_{2}$ and $\mathrm{CO}_{2}$ (according to equation 1), as found in several investigations by final product analysis and by molar balance calculations [9, 24, 36, 38a, 98]. A considerable number of studies have corroborated the primary dehydrogenation of alcohols into the corresponding ketones [59, 69-71, 80a, 99] or aldehydes [59, 64, 69-70, 100], thus adding support to this carbonyl route.

4.5.2. Carboxylic acid photoreforming mechanisms. Both mechanisms suggested above (stepwise dehydrogenation and non-selective dissociation of alcohols, represented by Figure 15 and Figure 16, respectively) are consistent with the formation of $\mathrm{H}_{2}, \mathrm{CO}_{2}$ and alkanes from several oxygenated substrates. However, their postulation was only based on qualitative gas phase sample analyses, whilst (intermediate) by-products such as ketones, aldehydes or acids should be expected to reside mostly in the liquid phase, and adsorbed or transient species such as alkyl radicals might be found on the surface of the photocatalyst. Considering carboxylic acids as the substrates have proven as the basis of revealing studies to elucidate general photoreforming mechanisms.

Detection of methyl radicals from acetic acid by spectroscopic techniques [68a, 87-88] is consistent with the route proposed by $\mathrm{Fu}$ et al. (Figure 16) entailing the oxidative $\mathrm{C}-\mathrm{C}$ cleavage of ketones and the decarboxylation of carboxylic acids into alkyl radicals and $\mathrm{CO}_{2}$. Also consistent with this mechanism, the photocatalytic carboxylic acid reforming produced, among diverse reaction products, alcohols of lower number of carbon atoms. This was rationalised by a formidably complex reaction mechanism which contemplates the decarboxylation of the acids into $\mathrm{CO}_{2}$ and alkyl radicals, and subsequent coupling of the latter with $[\mathrm{OH}]^{*}$ to generate the corresponding lower alcohol [37]. Such processes were favoured at high $\mathrm{pH}$ values, presumably promoted by the hydroxyl radicals formed by oxidation of the ubiquitous $[\mathrm{OH}]^{-}$present in basic media. A complete reaction network, consistent with the carbonyl route presented above, was postulated (Figure 17). 
4.5.3. Proposal of a consolidated photoreforming mechanism. In order to consolidate the mechanisms, a general route is presented in Figure 18, aiming at fitting most experimental results published in the literature. The proposed route has been devised in a "helical" cyclic way, so as to account for the reforming of any complex poly-oxygenated molecule, including polyols and saccharides, via a sequence whereby hydroxyl groups transform into carbonyl, which in turn oxidise to carboxylate residues. As side reactions on the reductive side, alkyl radicals may couple with adsorbed hydrogen atoms or with other alkyl radicals. This accounts for the formation of alkanes.

If one starts with an alcohol, the first suggested step is the dehydrogenation to form a 1hydroxyalkyl-type radical, which would undergo a second dehydrogenation to form the corresponding carbonyl compound. Dissociation of the latter would derive in a carboxylic acid and an alkyl radical. The exact way in which this step may proceed is still unclear. An oxidative C-C cleavage leading to a transient acyl cation, which would rapidly evolve to the acid in the presence of water, is suggested. The acid may decarboxylate into $\mathrm{CO}_{2}$ and a free alkyl radical, a process that can take place oxidatively. Alkyl radicals could be oxidised by, for example, $[\mathrm{OH}]^{\bullet}$ species. This would result in new, shorter-chain alcohols, which could become involved in a new reforming cycle. This is the basis of the suggested route (Figure 18) and is consistent with the experimentally observed complete reforming of several substrates, or with the partial reforming into lighter oxygenates, as is the case for the formation of acetaldehyde from lactic acid on illuminated $\mathrm{Pt} / \mathrm{TiO}_{2}$ [84]. A similar approach was used to rationalise the photoreforming of acetic acid on $\mathrm{Pt} / \mathrm{TiO}_{2}$ [61].

Regarding the formation of $\mathrm{H}_{2}, \mathrm{Li}$ and co-workers proposed in their mechanism that the protons primarily derived from methanol oxidation steps on titania sites should migrate to Pt particles, where they would be reduced [65]. The presence of water accelerates these processes (and thus $\mathrm{H}_{2}$ generation), in principle by providing a continuous medium for proton conduction on the adsorption spheres of the photocatalyst [77].

In the proposed "helical" mechanism, oxidative decarboxylation of formic acid into $\mathrm{CO}_{2}$ and hydrogen atoms would be the latter step. This process may proceed via different pathways depending on the conditions, but unfortunately, it has been seldom investigated in detail. In a particular report, it was suggested that the oxidative scission of the $\mathrm{H}-\mathrm{C}$ (or D-C) bond promoted by visible light on CdS powders was the rate-limiting step, based on kinetic isotope effects [101]. In brief, the proposed route aims at consolidating the previously suggested mechanisms and being consistent with the experimental data on the spectroscopic identification of intermediates.

\subsection{Deactivation events}


Regarding deactivation phenomena, the persistence of adsorbed carbonates on photocatalyst surfaces has been suggested as a detrimental factor for the continuous production of $\mathrm{H}_{2}$ by photoreforming, although this limitation can be overcome by conducting the reaction under a flow of an inert gas. For example, $\mathrm{H}_{2}$ production by glucose reforming on $\mathrm{Bi}_{0.5} \mathrm{Y}_{0.5} \mathrm{O}_{4}$ under visible light was observed to stop after $2 \mathrm{~h}$, whereas the reaction rates could be restored by simply flushing with $\mathrm{N}_{2}$ [102]. Selli and co-workers quantified this effect, concluding that a more than two-fold increase in $\mathrm{H}_{2}$ production rates could be achieved by bubbling $\mathrm{N}_{2}$ on an irradiated $\mathrm{TiO}_{2}$ suspension in aqueous methanol [103]. The extremely satisfactory results reported by Kondarides and co-workers on the complete sunlight-driven reforming of several biomass-derived oxygenates, including methanol [9, 38a], ethanol [9, 38a, 98], acetic acid [38a], glycerol [9, 24] and saccharides [9], were achieved by flowing Ar in order to continuously remove the gaseous products. Interestingly, conversion into $\mathrm{CO}_{2}$ proceeded essentially to completion on $\mathrm{Pt} / \mathrm{TiO}_{2}$ photocatalysts in those investigations, and thus, maximum $\mathrm{H}_{2}$ production was achieved according to equation 1 . In addition, flushing the $\mathrm{H}_{2}$ produced during the reaction would minimise the extent of back reactions, as for example the hydrogenation of carbonyl compounds [104]. Spectroscopic studies aiming at elucidating the mechanism of those transformations are scarce, and therefore, much needed in order to understand the underlying catalysis and to drive further progress in the field.

Based on the aforementioned experimental evidence, adsorbed intermediates or products (most notably carboxylate and carbonate species) on the photocatalyst surface may inhibit complete photoreforming. Notwithstanding this, these processes appear to be reversible, and desorption by simply keeping an inert gas flow through the reaction medium has been suggested to readily regenerate surface active sites for further reaction. Given the mild conditions involved, side reactions are minimised and photoreforming may proceed to completion with minimal deactivation.

\section{Photocatalysts for $\mathrm{H}_{2}$ production from oxygenated substrates}

Most of the photocatalysts used for the evolution of $\mathrm{H}_{2}$ from aqueous systems are solid materials composed of a light-absorbing semiconductor, and one or more metallic co-catalysts. The role of the semiconductor is to enable the generation of charge carriers (electrons and holes) upon light absorption, and their transport to the surfaces of the material, where redox processes take place. In this context, the match of the band gap of the semiconductor with the energy of incident photons is of utmost importance, that is, only wavelengths shorter than the edge of absorption of the material as defined by its band gap will cause promotion of an electron from its valence to its conduction band. Furthermore, electron transfer from the conduction band to an acceptor and from a donor to 
the valence band - hole annihilation — will be possible only if the energy positions are higher and lower, respectively, than those of the corresponding redox pairs. This, as illustrated in Figure 19 for the water splitting reaction relative to the band positions of a range of different semiconductors, allows the prediction and rationalisation of photocatalytic processes. Co-catalysts are generally incorporated on the surface of the semiconductor to provide active sites for the evolution of hydrogen gas by reduction of protons or related species, thus improving efficiencies. On the side of oxidation half reactions, the surface of the semiconductor itself has been proven to catalyse the transfer of holes to oxygenated substrates - or, in other words, the transfer of electrons from the oxygenated substrate to the semiconductor with concomitant annihilation of the photo-generated holes. The latter fact is reminiscent of the use of semiconductor photocatalysts for the aerobic oxidation of organic substances [66a].

Among semiconducting materials, $\mathrm{TiO}_{2}$ is the most suitable, due to its chemical and photochemical stabilities, and low cost. Many other semiconductors, including simple oxides, mixed oxides, oxynitrides, nitrides or sulfides have been also investigated. Furthermore, nanocomposites based on more than one kind of semiconductor have also received notable attention. These different photocatalysts will be described in detail in the following sections. Recently, more sophisticated catalytic systems (e.g. hybrid homogeneous-heterogeneous, biomimetic) have also been studied, and they are mentioned in a further section. With regard to co-catalysts, a dedicated section, focusing on the methods of incorporation onto the main material, their morphology and their chemical features, is also included. In this section, an outline-mainly focused on synthesis and performance-of photocatalytic materials for $\mathrm{H}_{2}$ production from oxygenated substrates is provided.

\subsection{Photocatalysts based on $\mathrm{TiO}_{2}$}

Titanium dioxide is largely the preferred semiconductor material for the design of heterogeneous photocatalysts and photo-anodes. The abundant literature on $\mathrm{TiO}_{2}$ photocatalysts for $\mathrm{H}_{2}$ production $[7 \mathrm{a}, 23,105]$ has been extensively reviewed elsewhere. Such a widespread use of $\mathrm{TiO}_{2}$ initially boosted after the discovery of the light-promoted electrochemical water dissociation in a system consisting on a rutile photo-anode and a platinum black cathode, i.e. the Fujishima-Honda effect, at the beginning of the 1970s [106]. Several years later, Bard and co-workers observed that aliphatic carboxylic acids underwent fast decomposition on illuminated aqueous $\mathrm{Pt} / \mathrm{TiO}_{2}$ suspensions $[89 \mathrm{a}$, 107]. During the following years, the efficiency of such simple experimental set-up was found general for the light-induced production of $\mathrm{H}_{2}$ from aqueous systems containing a wide variety of oxygenated substrates such as alcohols, carboxylic acids, aldehydes, saccharides, biopolymers, and 
even raw biomass feedstocks [36-37, 43, 100b, 104, 108]. Thereinafter, several of those oxygenated organic substances have been widely used for the evaluation of the $\mathrm{H}_{2}$-evolving activity of novel $\mathrm{TiO}_{2}$-based photocatalysts. This wave of research efforts has furnished the field of photocatalysis with a plethora of different $\mathrm{TiO}_{2}$ materials of notable performance. Part of the success of these systems stems from the high photoactivity of $\mathrm{TiO}_{2}$ for the oxidation half-reactions [6a, 66a].

The many titanium dioxide materials studied for the photocatalytic production of $\mathrm{H}_{2}$ exhibit rather dissimilar activities, as recently demonstrated for a range of $\mathrm{TiO}_{2}$ commercial samples [109], and these differences in performances are caused by a number of diverse factors. Among these factors, some of the most important are related to the features of the $\mathrm{TiO}_{2}$ component itself, including structure, crystallinity, phase composition, particle size, surface area, morphology or chemical modification (doping, defects). In general terms, and as for other semiconductors, high crystallinity and large surface area are desirable for enhancing performance, due to the reduced tendency to electron-hole recombination, and to the availability of surface active sites.

5.1.1. Synthesis of $\mathrm{TiO}_{2}$ materials with different phase structures. Titanium dioxide occurs mainly as four different crystalline polymorphs: anatase, rutile, brookite and $\mathrm{TiO}_{2}(\mathrm{~B})$ [7a]. In addition to its crystalline forms, amorphous $\mathrm{TiO}_{2}$ may be also obtained by hydrolysis of titanium alkoxides [110]. A wide variety of synthetic methodologies for the preparation of titania materials containing different polymorphs or morphologies is available in the literature [111].

Rutile can be generally obtained by annealing any of the other polymorphs (generally anatase) at high temperatures [89a, 111b, 112]. These processes usually entail particle growth and consequently, surface area reduction. On the other hand, crystallinity is in parallel enhanced at increasing temperatures. These phenomena were illustrated by Kudo and Miseki (Figure 20). In fact, it has been demonstrated that the thermodynamic stability of the different phases is dependent on surface area: whilst anatase is the phase of lowest enthalpy for particle sizes below $c a .40 \mathrm{~nm}$, brookite is more stable at intermediate sizes and rutile becomes thermodynamically favoured above $200 \mathrm{~nm}$ [111b]. Regarding photocatalytic activities, direct comparisons between the different structures cannot be formulated in absolute terms, since it proves impossible to prepare analogues of identical textures, surfaces and morphology. Furthermore, the extent at which distinct facets are exposed in crystallites of particular phases may also have influence adsorption phenomena, and consequently, reaction rates. In spite of these fundamental difficulties, it is widely accepted that anatase is the most photoactive $\mathrm{TiO}_{2}$ structure, followed by rutile and $\mathrm{TiO}_{2}(\mathrm{~B})$ [108d, 113], The higher activity of anatase as compared to rutile, by two orders of magnitude, has been proven for the production of $\mathrm{H}_{2}$ from ethanol under UV light [114], whereas amorphous titania solids exhibit 
little activity, presumably due to the ubiquity of defects promoting electron-hole recombination [48]. A comparative study by Waterhouse and co-workers examined the photocatalytic $\mathrm{H}_{2}$ production activity of different $\mathrm{Au} / \mathrm{TiO}_{2}$ materials from several alcohols in water under UVA light; their data led to the following rank: anatase/rutile > anatase > brookite > rutile [115]. In other studies, superior performance of brookite phases as compared to anatase for alcohol photoreforming under UV-vis irradiation has been suggested [116]. Some studies have concluded that rutile is more photoactive than anatase under UV light [112]. Under UV-free irradiation, platinised rutile is more active than anatase (albeit the reported rutile samples contained minor amounts of anatase which could favour charge separation due to the phase junction effect, as discussed in the following section) [117]. This was ascribed to the more favourable visible light absorption of rutile, according to its narrower band gap (3.0 eV), as compared to those of anatase $(3.2 \mathrm{eV})$ or brookite $(3.1-3.3 \mathrm{eV})$ [7d, 118]. One of the reasons claimed to be behind the lower activity of rutile lies in its direct band gap characteristics, as compared to the indirect band gaps for anatase and brookite [115].

5.1.1.1. Anatase. The most common synthetic route towards anatase is the annealing of amorphous $\mathrm{TiO}_{2}[48,110,119]$. Similarly, sol-gel procedures can be followed to generate titanium dioxide precursors for the synthesis of anatase [61, 96, 115, 120], occasionally, together with minor amounts of brookite [38b, 96, 120c] and/or rutile [96, 120b, 120c]. This transition occurs at moderate temperatures $\left(>300^{\circ} \mathrm{C}\right)$ without significant sintering [48]. Anatase can be also obtained by wet methods in aqueous media under a wide variety of conditions [38b, 96, 115, 121]. Particular examples of this include the formation of anatase nanotubes prepared by hydrothermal methods and subsequently loaded preferentially at their interior with gold using vacuum-assisted impregnation [122], or the hydrothermal treatment of titania precursors in the presence of ethanol at $160{ }^{\circ} \mathrm{C}$ for $16 \mathrm{~h}$ [115]. The growth of anatase crystallites usually results in preferential exposure of the most thermodynamically stable $\{101\}$ facets, although this pattern can be modified in high concentrations of fluoride under hydrothermal conditions, which favour the formation of bipyramids truncated by $\{001\}$ planes [123].

5.1.1.2. Brookite. Mixed materials containing variable amounts of brookite, anatase and rutile have been prepared by several methods, including hydrothermal treatment of sodium titanates [124] or titania [116b], or sol-gel synthesis followed by annealing [120c, 125]. Isolation of brookite from rutile by peptisation of the former into colloidal sols has been demonstrated [116b, 125]. Methods for producing pure brookite are scarce [116b]. Among these, significant success was reported by a hydrothermal route using a water-stable titanium complex and high concentrations of urea as a source of $[\mathrm{OH}]^{-}$, which lead to the production of brookite nanorods, whereas materials obtained 
with lower amounts of this additive favoured the formation of anatase/brookite mixtures, pure anatase nanoparticles [121b], and/or rutile [126]. Directed synthesis of brookite was achieved by a similar procedure from a tetranuclear titanium complex resembling the lattice building units of the final structure in basic media, whereas lower $\mathrm{pH}$ values lead to rutile or rutile/anatase nanomaterials [127]. In acidic media, slight modification of experimental conditions may direct towards the preferential growth of a particular polymorph from amorphous $\mathrm{TiO}_{2}$. For example, anatase nanoparticles can be obtained at $200{ }^{\circ} \mathrm{C}$ in acetic acid $(1.5 \mathrm{M})$ solutions, whereas a stronger acid such as $\mathrm{HCl}$ favours, depending on the temperature, the production of rutile nanorods $\left(200{ }^{\circ} \mathrm{C}\right)$ or brookite nanoparticles $\left(175^{\circ} \mathrm{C}\right)$ [118]. By starting from $\mathrm{TiCl}_{3}$, pure brookite nanoparticles can be obtained by air oxidation under controlled heating, with rutile contamination becoming significant otherwise [116a].

5.1.1.3. $\mathrm{TiO}_{2}(\mathrm{~B})$. The growth of $\mathrm{TiO}_{2}(\mathrm{~B})$ phases is favoured by mild calcination $\left(<500{ }^{\circ} \mathrm{C}\right)$ of hydrogen titanates $\left(\mathrm{H}_{2} \mathrm{Ti}_{x} \mathrm{O}_{x+1}\right)$, which can be in turn prepared from a range of titania sources under strongly basic hydrothermal conditions, generally in the presence of high concentrations of $\mathrm{NaOH}$ (leading to the formation of sodium titanates, $\mathrm{Na}_{2} \mathrm{Ti}_{x} \mathrm{O}_{x+1}$ ), and subsequent ionic exchange with Brønsted acids $[113,128]$. In these cases, both the hydrogen titanate and the final $\mathrm{TiO}_{2}(\mathrm{~B})$ materials show one-dimensional morphologies such as nanofibers, nanowires or nanorods (diameter: 10$40 \mathrm{~nm}$; length: $\left.10^{2}-10^{3} \mathrm{~nm}\right)$. After annealing at higher temperatures, the one-dimensional morphologies can be maintained, despite the preferential formation of the anatase phase [129]. Similar procedures have resulted in the direct transformation of hydrogen titanate into anatase nanotubes, which in turn tend to collapse as nanorods or monodimensional nanoparticles at increasing temperatures [130]. In addition to the aforementioned hydrothermal/annealing procedures, solvothermal methods can be also designed for the synthesis of $\mathrm{TiO}_{2}(\mathrm{~B})$ nanosheets with exposed $\{010\}$ facets [131].

5.1.1.4. Rutile. In addition to the well-known calcination procedures, rutile can be also obtained by lower temperature methods. For example, Harada and Ueda reported the synthesis of rutile nanoparticles (sizes below $12 \mathrm{~nm}$ ) in acidic aqueous media $(\mathrm{HCl}, 0.5 \mathrm{~N})$ at $80{ }^{\circ} \mathrm{C}$ [132]. Harsher acidic hydrothermal conditions $\left(\mathrm{HCl}, 4 \mathrm{~N}\right.$ at $\left.175^{\circ} \mathrm{C}\right)$ also result in selective rutile formation, albeit larger particles (57 $\mathrm{nm}$ average diameters) were observed [115]. Hydrothermal syntheses can be also directed towards rutile formation from a titanium lactate precursor [126]. Room temperature sol-gel has also been reported for the synthesis of nanoparticulate rutile [133].

5.1.2. Synthesis of $\mathrm{TiO}_{2}$ materials with different morphologies. Tailoring the morphology of $\mathrm{TiO}_{2}$ is an extremely active field of research. A series of dedicated reviews on the synthesis of 
titania nanoparticles [111a], nanotube arrays [134], nanowires/nanorods/nanobelts [135], nanosheets [136], three-dimensional suprastructures [137], or crystals with preferentially exposed facets [138] have recently seen the light.

5.1.2.1. Mesoporous $\mathrm{TiO}_{2}$. The synthesis of hexagonally packed mesoporous titania by a surfactanttemplated sol-gel method was first reported by Antonelli and Ying in 1995 [139]. These authors concluded that a strict control of reaction conditions, including $\mathrm{pH}$, surfactant type or additive (acetylacetone) concentration, was needed for successful mesoporosity formation. However, these materials are not hydrothermally stable. More straightforward preparation methodologies have led to materials exhibiting limited and disordered (inter-particle) mesoporosity [140], and relatively high surface areas $\left(50-100 \mathrm{~m}^{2} \mathrm{~g}^{-1}\right)$ of the resulting materials have proved in some cases to be beneficial for photocatalytic hydrogen production as compared to reference $\mathrm{TiO}_{2}$ materials [140a]. In this regard, the generation of $\mathrm{Pt} / \mathrm{TiO}_{2}$ aerogels by treatment of sol-gel precursors under supercritical $\mathrm{CO}_{2}$ has been reported as a successful method to maintain high specific surface areas (120-160 $\mathrm{m}^{2} \mathrm{~g}^{-1}$ ), even after annealing at $500{ }^{\circ} \mathrm{C}$; the resulting materials showed enhanced photocatalytic $\mathrm{H}_{2}$ production activities from aqueous ethanol under UV-visible light [141].

5.1.2.2. One-dimensional $\mathrm{TiO}_{2}$ materials. In addition to the growth of $\mathrm{TiO}_{2}$ nanowires or nanofibers from titanates and other synthetic methodologies, one-dimensional titanium dioxide can be obtained by a range of other methods. It is worth noting that electrospinning techniques have been applied to the synthesis of $\mathrm{TiO}_{2}$ nanofibers formed by bundles of nanofibrils (diameters: 15-75 nm). These one-dimensional nanomaterials adopted anatase or mixed anatase/rutile structures after calcination, and proved relatively efficient for the photoreforming of aqueous methanol [142].

5.1.2.3. $\mathrm{TiO}_{2}$ nanotube arrays. The formation of $\mathrm{TiO}_{2}$ nanotube arrays has been reported by electrochemical (potentiostatic) anodisation of titanium metal foils [143]. This procedure is becoming increasingly popular for the preparation of photocatalytic films or electrodes, due to their self-standing planar configuration which may enable effective and uniform illumination, and consequently, enhanced light harvesting, the large proportion of their exposed areas, and the possibility for vectorial charge transfer between interfaces [134]. Materials based on these nanotubes have been applied to the production of $\mathrm{H}_{2}$ by photoreforming in some occasions. Highly ordered, hexagonally packed $\mathrm{TiO}_{2}$ nanotube stumps (exhibiting high diameter-to-height ratio) formed in acidic electrolytes were amorphous after the anodisation treatment, although annealing resulted in crystallisation into anatase and rutile phases while keeping the original morphology [143a]. Preferential deposition of metals (gold) onto the top part of the nanotubes was achieved by sputtering methods, and the resulting materials were successfully used for the photoreforming of 
aqueous ethanol [143a]. Loading of metals can be also performed during the anodisation process, as reported for $\mathrm{Cu}_{2} \mathrm{O}$, which can be deposited onto $\mathrm{TiO}_{2}$ nanotube arrays by adding copper salts to the anodisation electrolyte [144]. On the other hand, incorporation of iron within the framework of titania in similar materials has been reported [145].

5.1.3. Phase junction engineering in $\mathrm{TiO}_{2}$ photocatalysts. Phase junctions are commonly understood as intimate physical contacts between two different polymorphs of the same material. In the case of semiconductors such as $\mathrm{TiO}_{2}$, these contacts may enable charge carrier mobility from one of the polymorphs to the other. In nanoparticulate photocatalytic systems, there is general consensus about the positive effects of charge transport across crystallite boundaries on reducing electron-hole recombination.

5.1.3.1. Anatase/rutile nanocomposites. Phase junctions in $\mathrm{TiO}_{2}$ materials may be generated by the controlled transformation of one of the polymorphs into another, for example by thermal annealing, or, in the case of the well-known reference AEROXIDE ${ }^{\circledR} \mathrm{TiO}_{2} \mathrm{P} 25$ (hereinafter referred to as P25) material [146], by single-step flame pyrolysis, a method leading to a composite of anatase and rutile nanoparticles and minor amounts of amorphous titania [77, 100c, 103, 147]. Other direct methods of preparing materials exhibiting anatase-rutile phase junctions include suspension plasma spraying [148].

According to the pioneering studies by Bard and co-workers, the photoactivity of anatase materials improved upon a self-doping treatment under hydrogen atmosphere at $650{ }^{\circ} \mathrm{C}$, which caused the transformation of $c a .10 \%$ of the initial phase into rutile [89a, 107]. However, complete formation of rutile at $1100{ }^{\circ} \mathrm{C}$ rendered significantly less active photocatalysts. Several reasons (conductivity, existence of surface states, position of Fermi level) were postulated for the increased photoactivity of such so-called self-doped $\mathrm{TiO}_{2}$. The most widely accepted hypothesis for the enhanced photocatalytic activity of nanocomposite anatase/rutile materials is based on charge separation across the junction between crystallites of either phase.

A number of examples in the literature have further confirmed that the presence of both anatase and rutile nanocrystals in intimate contact is related to the high photoreforming efficiency of materials based on $\mathrm{TiO}_{2}$ [108d, 133, 149]. Most likely for this reason, the commercially available P25 is acclaimed by many as one of the most photocatalytically active titanium dioxide materials known and a desirable benchmark [42]. Ohno et al. studied the morphology of P25 by transmission electron microscopy (TEM) and concluded that segregated agglomerates of either anatase or rutile nanoparticles (diameters $\approx 25$ and $85 \mathrm{~nm}$, respectively) existed in the pristine powder, whereas they tended to separate and closely blend with each other by dispersion in liquid media by sonication 
[149d]. After selectively isolating rutile or anatase from P25, Jovic et al. observed that photocatalysts prepared from either crystalline phase performed less efficiently than the original material, confirming the synergistic effect of the mixed phases [150]. This has also been confirmed for the $\mathrm{H}_{2}$ production from methanol on $\mathrm{TiO}_{2}$ nanomaterials composed of anatase and rutile at varying compositional ranges prepared by flame pyrolysis, whereby phase junctions were formed at an intimate inter-particle level [147]. Although some authors question whether an actual synergy takes place [42], there is wide consensus about efficient charge separation across anatase-rutile phase junctions being responsible for the notable photocatalytic activity of P25 and other similar $\mathrm{TiO}_{2}$ nanomaterials [149a]. The main point of discrepancy among researchers in this field is in which direction (anatase-to-rutile or vice versa) electrons are preferentially transferred. A number of studies claim that more photo-generated electrons flow from anatase to rutile, according to the presumably lower energy of the conduction band for the latter [147, 149a, 149b]. However, a recent study combining materials simulation techniques and X-ray photoemission experiments support the hypothesis of photo-generated charge separation and increased lifetime by electron flow from rutile to anatase through inter-phase junctions (Figure 21) [151]. Other factors, often resulting in opposing effects, come into play for samples treated at high temperature. Along these lines, it has been demonstrated that upon heat-induced partial transformation of anatase to rutile, activity is increased when normalised per surface unit, albeit decreased on a mass basis due to sintering and reduced external area [152].

5.1.3.1. Other phase-junction $\mathrm{TiO}_{2}$ materials. In addition to the extensively studied anatase/rutile nanocomposites, titanium dioxide materials having intimately contacted $\mathrm{TiO}_{2}(\mathrm{~B})$ nanotubes and anatase nanoparticles have also been proven to exhibit high photocatalytic activity for hydrogen production from ethanol [113b]. Other works showed evidence along the same lines about the benefits of anatase formation on $\mathrm{TiO}_{2}(\mathrm{~B})$ nanowires by controlled annealing, in these cases for aqueous methanol as the substrate for photocatalytic $\mathrm{H}_{2}$ production [113a, 128d]. In these works, it was argued that the presence of phase junctions between both crystalline $\mathrm{TiO}_{2}$ forms might be responsible for efficient charge separation and, in consequence, high photocatalytic activity, even outperforming P25. One of these reports thoroughly investigated the charge transfer mechanisms across the junction by EPR spectroscopy, and concluded that electrons flow from anatase to $\mathrm{TiO}_{2}$ (B) [128d]. Finally, anatase/brookite materials prepared by hydrothermal treatment of P25 displayed superior ethanol photoreforming activity (when normalised relative to surface area) than a reference titania material synthesised by a sol-gel method, a fact which was ascribed to a larger amount of active sites in the nanocomposites with anatase-brookite junctions [124]. Hydrothermal 
transformation of a titanium lactate precursor in the presence of urea under may also lead to anatase/brookite materials exhibiting high ethanol or glycerol photoreforming activity [126].

5.1.4. Heterojunction photocatalysts based on $\mathbf{T i O}_{2}$. Due to the limited visible light absorption capacity of $\mathrm{TiO}_{2}$, but keeping in mind its stability and favourable kinetics for charge transfer processes onto adsorbed species, numerous research projects have been devoted to combine, at the nanometric level, this semiconductor with others which may enhance activity under wavelengths longer than the absorption edge of titanium dioxide $(c a .400 \mathrm{~nm})$. The role of the second semiconductor may be viewed in these composite materials as that of a photosensitiser. Accounts on this topic can be found in dedicated reviews [153]. Herein, aspects on the preparation of nanocomposites consisting of $\mathrm{TiO}_{2}$ and at least another semiconductor, and on their performance for photocatalytic hydrogen production from biomass-derived feedstocks, are concisely described.

As a semiconductor exhibiting strong visible light absorption, cadmium sulfide has been frequently employed in photocatalytic systems. Coupling $\mathrm{CdS}$ with $\mathrm{TiO}_{2}$, provided that electrons and/or holes can be transferred across heterojunctions between them, is in principle an interesting strategy to achieve activity under visible light. In this context, Lianos and co-workers developed films consisting on separated cathodic and anodic areas on conductive fluorine-doped tin oxide layers; on the anodic part, $\mathrm{CdS}$ was deposited on a $\mathrm{TiO}_{2}$ layer, and it was observed that the dependence of photoactivity (hydrogen production form aqueous ethanol) of the resulting system with incident wavelength approximately matched the absorption spectrum of the sulfide material, thus suggesting that light absorption on CdS and subsequent charge separation might activate the reaction [154]. However, $\mathrm{Pt} / \mathrm{CdS} / \mathrm{TiO}_{2}$ analogue materials in suspension did not perform efficiently for ethanol as the substrate [154b]. Enhancement of visible light activity was also demonstrated, in the particular case of $\mathrm{H}_{2}$ production from aqueous lactic acid, on $\mathrm{Pt} / \mathrm{TiO}_{2}$ sensitised with $\mathrm{CdS}$ by successive impregnation with $\mathrm{Cd}(\mathrm{II})$ and sulfide solutions [155]. Mighri, Do and co-workers prepared $\mathrm{TiO}_{2}$ nanorods with deposited $\mathrm{CdS}$ as the photocatalytic support for nickel nanoparticles, and the resulting materials used for ethanol photoreforming [156]. A related procedure was also performed for the CdS sensitisation of titanate nanodisks [157].

A recent strategy to favour the separation and transport of the charges generated in $\mathrm{TiO}_{2}$ upon irradiation is the use of mediator materials which contact both the semiconductor and the active sites for charge transfer reactions, as opposed to the more conventional direct deposition of the cocatalyst. Although probably not accurately classified as heterojunctions, the close interaction between semiconductor and such mediators has been claimed to enable efficient charge transport. Among these electron mediators, highly conducting carbon materials such as graphene [158], 
carbon nanotubes [159] or nanocones [160] are the most prominent examples. The high dispersion ability of carbon nanocones has enabled the preparation of titania-based photocatalysts whereby intimate contact between the carbon and $\mathrm{TiO}_{2}$ phases results in high ethanol photoreforming activity and long durability [160]. It should be noted, though, that decomposition by actual reforming of graphitic carbon or hydrocarbons is possible under appropriate irradiation conditions, as proved elsewhere [161].

5.1.5. Doped $\mathrm{TiO}_{2}$ for enhanced visible light absorption. In classic semiconductor theory, doping means introduction of structural or electronic modifications to the material, such that additional electronic states are thereby created between the valence and conduction bands [162]. In the context of photocatalysis, the promotion of electrons to higher states can be thus supplied by photons of lower energy (i.e., longer wavelength) for doped semiconductors than for the pure pristine materials. For this reason, it is not surprising that a number of research teams have delved into the preparation of doped titania materials which can be activated by visible light. Three different strategies have been implemented for conferring visible light activity to $\mathrm{TiO}_{2}$ : $(i)$ introduction of metals, (ii) introduction of non-metals, and (iii) partial reduction. In all cases, modification of $\mathrm{TiO}_{2}$ is preferentially done to the bulk of its structure (so as to ensure homogeneous distribution of the newly created electronic states) and at relatively low (in some instances called impurity) levels. In spite of the fact that doping $\mathrm{TiO}_{2}$ usually results in a coloured material with a red-shifted absorption edge, not always this guarantees the effective and homogeneous electronic modification of the material, since in some cases absorption can be exclusively caused by isolated (parasitic) sites, not contributing to the bulk electronic bands, as is the case for certain $\mathrm{d}-\mathrm{d}$ transitions [163]. The many reports on doped $\mathrm{TiO}_{2}$ have focused on a wide range of applications for the final material, as extensively reviewed elsewhere [7a, 7e, 153b, 163-164]. Herein, mainly those dealing with photocatalytic reforming for enhanced production of $\mathrm{H}_{2}$ under visible light are summarised.

5.1.5.1. Metal-doped $\mathrm{TiO}_{2}$. The introduction of metal dopants into $\mathrm{TiO}_{2}$ is generally achieved during the primary growth of the structure by providing the appropriate metal cations to the synthetic media, although post-synthesis methods have also been reported [153b]. In principle, metal atoms in a 4+ oxidation state can replace Ti(IV) atoms without altering the electroneutrality of the material, whereas introduction of lower oxidation state metals should be accompanied with the formation of oxygen vacancies, and conversely, metal atoms in $\geq 5+$ oxidation states are expected to require additional oxygen atoms in order to maintain bulk charge balance. Further to the charge constraints, ionic radii are an important factor dictating the compatibility of the dopant metal with the matrix 
structure, and often, miscibility is limited up to a maximum content, above which, phase segregation can occur. It has been frequently postulated that despite enhancing light absorption ranges, single metal doping and the related formation of oxygen defects may facilitate charge recombination, and thus, result in decreased photocatalytic activities [5d].

A few reports have been devoted to the enhancement of photoreforming efficiencies by metal doping of $\mathrm{TiO}_{2}$. For example, Sun et al. prepared $\mathrm{TiO}_{2}$ materials containing $\mathrm{Fe}$ and/or Ni by solvothermal methods and subsequent annealing [165]. The same authors added Ag to similar synthetic procedures [166]. They found that, whilst $\mathrm{Fe}$ and $\mathrm{Ni}$ were incorporated into the bulk structures (as suggested by shifts in Raman bands) [165-166], Ag was segregated in the form of metallic nanoparticles during the high temperature treatment, and both modifications lead to enhanced $\mathrm{H}_{2}$ production from aqueous ethanol under light from a Xe lamp [166]. Samokhvalov and

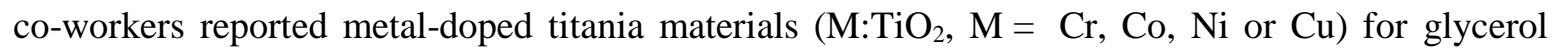
photoreforming under Hg lamp light, and observed that the activity was dependent on the metal in the following order: $\mathrm{Cr}<\mathrm{Co}<\mathrm{Ni}<\mathrm{Cu}$ [167]. This increasing trend in activity was ascribed to decreasing electron-hole recombination, as observed to reduced photoluminescence [167]; similar reasoning was put forward in the aforementioned works [165-166]. The group of Saif, Mozia, et al. investigated on the incorporation of low amounts $(\leq 0.07 \%$ mol relative to Ti) of lanthanides (Eu or $\mathrm{Sm})$, which were in part believed to form segregated oxides. They observed the increased activity of the doped materials, relative to P25 as a reference, for the decomposition of aqueous acetic acid under UV-rich light, with preferential formation of $\mathrm{CH}_{4}$ and $\mathrm{CO}_{2}$ [168].

5.1.5.2. Non-metal-doped $\mathrm{TiO}_{2}$. Modification of titanium dioxide with low concentrations of nonmetals is considered to render materials with enhanced visible light absorption capabilities due to the formation of electronic states between the valence and conduction bands of the parent structure, and to the corresponding reduction of the band gap, as stated in a seminal report by Asahi and coworkers [169]. Based on theoretical calculations [169-170], it was anticipated that nitrogen doping would exert a more pronounced improvement of the activity under visible light due to a better overlapping of nitrogen-induced electronic levels with the top of the valence band, as compared to other elements such as fluorine, carbon, sulfur or phosphorus (Figure 22), yet its direct influence on the efficiency of proton reduction to $\mathrm{H}_{2}$ is questionable, since the conduction band position is not changed [170]. Nitrogen doping has become widely applied for the preparation of efficient titania materials able to harvest visible light [164c]. The fact that nitrogen is the most appropriate nonmetal dopant was experimentally confirmed by the more efficient photodegradation of gaseous acetaldehyde on materials doped by nitrogen sputtering techniques as compared to pristine $\mathrm{TiO}_{2}$ 
samples [169]. Annealing under $\mathrm{NH}_{3}$ (at temperatures ranging from 550 to $600{ }^{\circ} \mathrm{C}$ ) has also been reported as a method for producing $\mathrm{N}$-doped titania, and the resulting materials proved to be active for the decomposition of 2-propanol under visible light [171]. A similar N-doping method was performed on nanofiber materials, giving rise to anatase containing nitrogen atoms in either substitutional or interstitial positions, as suggested by shifts in XRD peaks, X-ray photoelectron spectroscopy (XPS) data or TEM [129]. Harsher conditions (annealing at $750{ }^{\circ} \mathrm{C}$ ) have been reported to induce substitution of oxygen with nitrogen and, eventually, partial transformation of $\mathrm{TiO}_{2}$ into TiN; an increase in $\mathrm{H}_{2}$ production photoactivity from aqueous ethanol was observed for these materials with increasing nitridation times [172].

In addition to nitrogen, other non-metals have been studied as $\mathrm{TiO}_{2}$ dopants. Carbon-doped anatase powders prepared by oxidative annealing of TiC proved to be active for the decomposition of gaseous 2-propanol under visible light $(400-530 \mathrm{~nm})$ [173]. The production of boron-doped anatase or rutile films prepared by chemical vapour deposition have proven (after platinum deposition onto them) to be more active for the UV-promoted ethanol photoreforming than non-doped analogues [174]. This was ascribed to boron in substitutional oxygen positions favouring the exposure of highly active $\{200\}$ facets. According to a reported sol-gel method, phosphorus can be introduced in the structure of $\mathrm{TiO}_{2}$ as a cationic dopant, and the resulting material was proven to exhibit extended visible light absorption ranges and glycerol photoreforming activity [175].

As opposed to boron, nitrogen, phosphorus or carbon as heteroatoms, sulfur is isoelectronic to oxygen and thus may replace $\mathrm{O}$ sites in $\mathrm{TiO}_{2}$ without altering the bulk electroneutrality of the material. One possible method for producing such sulfide-doped $\mathrm{TiO}_{2}$ exhibiting enhanced visible light absorption is pulsed laser desorption; after further Ni deposition, these films showed superior photoreforming activity than the undoped analogues [176]. As opposed to sulfide doping, the incorporation of sulfur as a cationic dopant $\left(\mathrm{S}^{4+}\right.$ and $\mathrm{S}^{6+}$ according to XPS data) to the titania structure by annealing in the presence of thiourea has been reported, although no details on possible nitrogen impurities were provided [177]. Such S-doped materials were claimed to be more active under visible light as compared to $\mathrm{N}$-doped (similarly prepared using urea instead of thiourea) or non-doped counterparts, albeit the reported $\mathrm{H}_{2}$ production rates were low $\left(<1 \mu \mathrm{mol} \mathrm{g}_{\mathrm{cat}}{ }^{-1} \mathrm{~h}^{-1}\right)$.

Theoretical calculations have revealed that non-metals (C, N or B) in substitutional positions tend to introduce states containing unpaired electrons above the valence band, and may favour the formation of defects, whereas the same atoms in interstitioal positions favour the donation of electrons and the formation of O-heteroatom species [170]. In general, the conduction band is barely affected by doping with non-metals, and hence, influence on $\mathrm{H}_{2}$ production should not be 
regarded as a direct consequence, but rather as result of altered oxidation processes. Furthermore, attention should be also paid to possible structural changes upon doping.

5.1.5.3. Self-doped $\mathrm{TiO}_{2}$. Several methods have been reported for self-doping of titanium dioxide, that is, for partially reducing the material with the formation of oxygen vacancies and $\mathrm{Ti}(\mathrm{III})$ centres. This can be achieved by $(i)$ thermal treatment under a reductive atmosphere [100f, 178], (ii) in the bulk of the material by combustion/pyrolysis methods [179], or (iii) by partial oxidation of titanium metal [180]. Strongly reductive conditions $\left(\mathrm{H}_{2}, 20\right.$ bar, $\left.200{ }^{\circ} \mathrm{C}, 5 \mathrm{~d}\right)$ give rise to the known "black $\mathrm{TiO}_{2}$ ", whereby $\mathrm{Ti}(\mathrm{III})$ and defect sites are selectively formed near the surface of anatase crystals in a disordered manner [178a]. After platinum deposition, the black $\mathrm{TiO}_{2}$ photocatalyst showed significant $\mathrm{H}_{2}$ production activity $\left(10 \mathrm{mmol} \mathrm{g}_{\mathrm{cat}}{ }^{-1} \mathrm{~h}^{-1}\right)$ by methanol photoreforming under simulated solar light. The enhanced visible light absorption observed for black $\mathrm{TiO}_{2}$ materials has inspired an extensive research activity in recent years [178b]. The generation of both $\mathrm{Ti}(\mathrm{III})$ and oxygen defects on gold- and/or platinum-loaded titania materials have been postulated, based on cyclic voltammetry data, under less harsh conditions (flowing $\mathrm{H}_{2}, 25^{\circ} \mathrm{C}, 1 \mathrm{~h}$ ), and the resulting materials proven to be active for the photoreforming of oxygenated substrates (ethanol or glycerol) under visible light [100f, 100g]. A contrasting report claims limited performance at room temperature of platinised black $\mathrm{TiO}_{2}$ for methanol photoreforming at wavelengths above $420 \mathrm{~nm}$; this was ascribed to kinetic limitations which could be overcome by increasing reaction temperatures [181].

\subsection{Photocatalysts based on oxides other than $\mathrm{TiO}_{2}$}

In their review on photocatalytic $\mathrm{H}_{2}$ production, Chen and co-workers stressed the appropriateness of oxide semiconductors based on metals in $\mathrm{d}^{0}$ or $\mathrm{d}^{10}$ configurations, among which $\mathrm{TiO}_{2}$ is the most frequently studied [7e]. As discussed above, the conduction band edge of $\mathrm{TiO}_{2}$ is marginally higher in energy than the $\mathrm{H}^{+} / \mathrm{H}_{2}$ redox potential, and band bending in solution may favour to a certain additional extent the $\mathrm{H}_{2}$ formation reaction. However, the reducing power of photogenerated electrons in the conduction bands of several other transition metal oxides is often insufficient for the $\mathrm{H}_{2}$ generation reaction to take place (e.g. $\mathrm{WO}_{3}, \mathrm{~V}_{2} \mathrm{O}_{5}, \mathrm{FeO}, \mathrm{CoO}, \mathrm{CuO}$ or $\mathrm{ZnO}$, see diagram in Figure 19) [182], or else, their band gaps too wide (e.g. $\mathrm{ZrO}_{2}$, Figure 19) [41]. Considering mixed

oxides, a significant deal of attention has been directed to titanium-based materials, chiefly titanates, which have conduction band edges above the reduction potential of the hydrogen ion [5d, 41]. Related photocatalysts such as vanadates, niobates or tantalates have also been studied. Prior art related to the use of these kinds of oxides for photoreforming reactions is summarised in this section. 
5.2.1. Simple oxides other than $\mathrm{TiO}_{2}$. Among oxides of metals in $\mathrm{d}^{0}$ configurations, tungsten(VI) oxide is an interesting as a semiconductor photocatalyst due to its favourable band gap for (partial) visible light absorption [183], although its conduction band edge is low for $\mathrm{H}_{2}$ production. In fact, Domen et al. described the lack of stability of $\mathrm{WO}_{3}$ due to photoreduction while attempting the dehydrogenation of 2-propanol [184]. However, $\mathrm{WO}_{3}$ has been proven to catalyse the lightactivated decomposition of oxalic acid into $\mathrm{CO}_{2}$ and $\mathrm{H}_{2}$ in the presence of $\mathrm{Cu}$ (II) [185]. Another substrate, more directly available from biomass processing, glycerol, can be fully photoreformed under visible light on $\mathrm{WO}_{3}$ modified with $\mathrm{Au}$ and Pt nanoparticles [186]. In this report, photo-action spectra clearly demonstrated that band gap excitation of both $\mathrm{WO}_{3}(<450 \mathrm{~nm})$ and $\mathrm{Au}(450-$ $600 \mathrm{~nm}$ ) were essential for the photocatalytic activity of the material. Sclafani et al. tested several insulators and semiconductors under strong UV-visible light for acetic acid decomposition and observed activity for most of them, including $\mathrm{ZnO}, \mathrm{TiO}_{2}$ and $\mathrm{WO}_{3}$, but not for $\mathrm{MoO}_{3}, \mathrm{MnO}_{2}, \mathrm{Cr}_{2} \mathrm{O}_{3}$ or $\mathrm{Fe}_{2} \mathrm{O}_{3}$ [44a]. For $\mathrm{SnO}_{2}$, a wide band gap (3.6 V) semiconductor, self-doping with $\mathrm{Sn}$ (II) [187], or co-doping with $\mathrm{Sb}$ and $\mathrm{Ce}$ [188], has been reported as a method to induce visible light activity.

The photoactivity of bare $\mathrm{ZnO}$ for $\mathrm{H}_{2}$ production from aqueous formaldehyde or methanol has also been reported recently [189]. Composites of $\mathrm{ZnO}$ nanorods grown on graphene have also proven active for the photoreforming of aqueous glycerol under Xe lamp light [190]. The performance of $\mathrm{ZnO}$ nanorod arrays under similar conditions could be enhanced by one to two orders of magnitude after formation of a $\mathrm{ZnS}$ shell during a hydrothermal treatment in the presence of thiosulfate anions [191]. Laser irradiation has been studied as a method to produce syngas from various alcohols using $\mathrm{NiO}$ as the photocatalyst [192]. The use of $\mathrm{Cu}_{2} \mathrm{O}$, which absorbs a significant portion of visible light due to its narrow band gap $(2.2 \mathrm{eV})$ [41], as a photocatalytic material has attracted some attention. Its activity for $\mathrm{H}_{2}$ production from aqueous formic acid or alcohols under UV-free light was demonstrated to be similar to that of $\mathrm{CdS}$, with the added advantage of suppressing $\mathrm{CO}$ formation [193].

In contrast to the aforementioned reports, which deal with photocatalysts in suspension, Fornasiero and co-workers have developed chemical vapour deposition methodologies for the preparation of nanostructured metallic oxide layers of a variety of materials, including $\mathrm{Cu}_{2} \mathrm{O}$ [194], $\mathrm{CuO}$ [194195], $\mathrm{ZnO}$ [195], $\mathrm{Fe}_{2} \mathrm{O}_{3}$ [196] or $\mathrm{Co}_{3} \mathrm{O}_{4}$ [197] as photocatalysts for $\mathrm{H}_{2}$ generation. These systems, despite being less known regarding mechanistic aspects, show great promise due to their high activities on a weight basis and the fact that no noble metals are needed [7c]. The authors argued that the unexpected activity of some oxides $\left(\right.$ e.g. $\left.\mathrm{Fe}_{2} \mathrm{O}_{3}\right)$ is due to a cathodic shift of their conduction band from having an insufficient energy for proton (or water) reduction, to a favourable reducing 
power [196b]. A similar method was applied Zhang et al. to produce $\mathrm{VO}_{2}$ films exhibiting photoactivity for ethanol photoreforming [198]. As a particularly interesting fact, the authors of this report claimed the growth of a new structure of the metal with significantly different band gap $(2.7 \mathrm{eV})$ to that of the previously known $\mathrm{VO}_{2}$ analogue $(0.7 \mathrm{eV})$.

5.2.2. Mixed oxides. 5.2.2.1. Titanates. Mixed oxides, and in particular those having perovskitelike structures, have been long considered for photocatalysis [105, 199]. Among these, titanates are the most frequently employed class of materials, given their favourable band edge positions (see Figure 19) [41]. They can be prepared by a variety of wet or solid-state procedures and from different precursors [199], including anatase as the titanium source [200]. Photocatalysts based on $\mathrm{SrTiO}_{3}$ as the light-harvesting component have been studied for the production of $\mathrm{H}_{2}$ from various oxygenated substrates [200-201], although the activity is restricted to UV radiation due to the relatively wide band gap of this semiconductor (3.40 V) [41]. Furthermore, Domen and co-workers demonstrated the efficiency of photocatalytic water splitting [202] and 2-propanol dehydrogenation [184] processes on $\mathrm{NiO} / \mathrm{SrTiO}_{3}$. In a comparative study, Zieliñska et al. established the order of activity of titanates $\left(\mathrm{MTiO}_{3}\right.$, where $\mathrm{M}$ is an alkaline earth metal) for the production of $\mathrm{H}_{2}$ from aqueous 2-propoanol under UV-rich light as follows: $\mathrm{Sr}>\mathrm{Ca}>\mathrm{Ba}$ [201d]. In general, titanate materials perform more modestly than most of the well-known titania-based photocatalysts (see Section 7 below). Mao and co-workers claimed higher $\mathrm{H}_{2}$ production rates from aqueous ethanol under UV-rich light, and using deposited platinum nanoparticles as co-catalyst, by introducing zirconium to calcium titanates (thus forming solid solutions, $\mathrm{CaTi}_{1-x} \mathrm{Zr}_{x} \mathrm{O}_{3}, x=0-0.15$ ) as compared to pure $\mathrm{CaTiO}_{3}$ [203]. The same authors also reported similar activities for photocatalysts based on $\mathrm{BaTi}_{4} \mathrm{O}_{9}[204]$.

5.2.2.2. Mixed oxides not containing titanium. Regarding vanadates, materials based on $\mathrm{BiVO}_{4}$ are the most interesting due to their visible light absorption ability. The photoreforming of glucose on yttrium-doped solids $\left(\mathrm{Bi}_{x} \mathrm{Y}_{1-x} \mathrm{VO}_{4}\right)$ [102] or alcohols on silver-modified $\mathrm{BiVO}_{4}$ has been reported [205]. Tantalates are also a class of materials occasionally proposed as semiconductors for photocatalysts, as in the case reported by Leung and co-workers dealing with active lanthanumdoped $\mathrm{MTaO}_{3}(\mathrm{M}=$ alkaline metal) materials for the photoreforming of glucose [206]. In their extensive investigations, Domen and co-workers reported the preparation of several niobates of layered structures, and their use for the photocatalytic production of $\mathrm{H}_{2}$. They observed activity from aqueous alcohols under $\mathrm{UV}$-visible light for $\mathrm{K}_{4} \mathrm{Nb}_{6} \mathrm{O}_{17}$ [207] or $\mathrm{Ca}_{2} \mathrm{Nb}_{3} \mathrm{O}_{10}$ [208]. Finally, it should be also pointed out that oxinitride semiconductors may also be regarded as active materials under visible light. Domen's group reported on the photoreforming of ethanol under irradiation of 
wavelengths above $420 \mathrm{~nm}$ using a photocatalyst based on platinum and ruthenium supported on $\mathrm{Y}_{2} \mathrm{Ta}_{2} \mathrm{O}_{5} \mathrm{~N}_{2}$ [209]. Tungstates, which have hitherto received limited attention as photocatalysts, are promising materials, as for example reported for glycerol photoreforming using $\mathrm{Bi}_{2} \mathrm{WO}_{6}$ without the need for additional co-catalysts [210]. Other classes of perovskite-like oxides including iron have been investigated as semiconductors responsive to visible light, as in the case of $\mathrm{Cu} / \mathrm{LaFeO}_{3}$ materials prepared by solid state synthesis and subsequent reduction, exhibiting activity for the photoreforming of formaldehyde [211].

\subsection{Photocatalysts based on sulfides and other chalcogenides}

The application of metal chalcogenides as light-absorbing components in quantum dot photovoltaic cells is a well-established topic of research. This is mainly due to their (generally) narrow band gaps, which cause visible light response [162]. Furthermore, their electronic states are higher in energy than those of oxides, and thus, the photo-excited electrons in their conduction bands are more strongly reducing (see Figure 19). Thus, it is not surprising that many researchers have dedicated significant endeavour to designing sunlight-active photocatalysts based on chalcogenides, (especially sulfides) and metals in $\mathrm{d}^{10}$ configurations for $\mathrm{H}_{2}$ production [212].

5.3.1. Cadmium sulfide. The most frequently employed among these solids is CdS, since it absorbs a significant part of the violet-blue-green irradiation $(<520 \mathrm{~nm})$. Both cubic and hexagonal phases of cadmium sulfide and related solid solutions can be prepared by appropriate syntheses [64]. The conduction band of CdS lies at more negative energies than that of proton reduction, and therefore, $\mathrm{H}_{2}$ generation is favourable, provided a suitable co-catalyst is deposited [41]. Direct electron transfer from photo-excited cadmium sulfide to platinum nanoparticles deposited on another, wide band gap, semiconductor (i.e. $\mathrm{TiO}_{2}, \mathrm{ZnO}, \mathrm{SnO}_{2}$ and $\mathrm{WO}_{3}$ ) led to identical activities in water/methanol mixtures under visible light, thus suggesting similar mechanisms. From this data, the possibility of electron transfer from CdS via oxide particles as mediators was discarded [182].

Despite its response to visible light, the appropriateness of $\mathrm{CdS}$ is jeopardised by photocorrosion events leading to oxidation of sulfide anions [212-213]. This can be circumvented in the presence of sacrificial electron donors, among which biomass derivatives are suitable, although aqueous sulfides perform better. The pioneering research of Sakata and Kawai demonstrated the suitability of CdS with supported platinum co-catalyst for the generation of $\mathrm{H}_{2}$ from aqueous alcohols or saccharides under UV-visible light [214]. Among a wide range of semiconductors tested, CdS was second in activity after $\mathrm{TiO}_{2}$, although it was the best performer when UV light was filtered off. They also extended the substrate scope to organic acids, mainly lactic [84, 215], and other multifunctional molecules [216]. Direct proof of the higher photoreforming activities of CdS as 
compared to $\mathrm{TiO}_{2}$ has been also recently observed for lactic acid as a substrate [217]. Domen et al. also proved the activity of bare CdS for the dehydrogenation of 2-propanol in water under UVvisible light [184]. Also during the early nineteen eighties, Tsubomura and co-workers reported efficient photoreforming of several oxygenates, including formic acid, methanol or formaldehyde on Pt/CdS [218]. Later, Jin and co-workers demonstrated that surface hydroxylation, which can be promoted by annealing under air, was essential for alcohol dehydrogenation under visible light [213a].

5.3.1.1. Enhancing the stability of CdS. Stability of CdS under irradiation is a common matter of concern. In this regard, cadmium photoreduction and, to some extent, leaching, were observed from CdS/zeolite composites during ethanol photoreforming under visible light [219]. In recent years, the most classic $\mathrm{Pt} / \mathrm{CdS}$ system has been studied for photocatalytic $\mathrm{H}_{2}$ production from formic acid [220], lactic acid [221], ascorbic acid [222] or glycerol [223] under visible light. In one of these

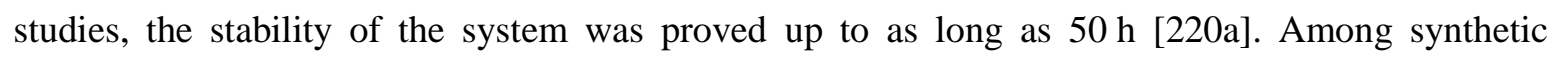
methods aiming at increasing the stability towards leaching, formation of a surface layer on $\mathrm{ZnS}$ proved beneficial over successive cycles of formic acid photoreforming [224]. Notwithstanding this, corrosion and agglomeration of materials based on $\mathrm{ZnS}$ has been reported [191a]. Another approach is the encapsulation of CdS nanoparticles in mesoporous silicate or aluminosilicate materials [220c, 225], although a certain degree of activity loss has been observed for reused photocatalysts [225b]. Metal organic frameworks (MOFs) have also been proposed as porous supports enhancing the resistance towards $\mathrm{Cd}^{2+}$ leaching [226]. The use of MOFs as electron mediator materials between CdS and Pt has also been reported [222]. Microwave-assisted synthetic methods provide unique opportunities for the generation of metal sulfide heterostructures with particular morphologies, as for example, those composed of $\mathrm{CdS}, \mathrm{MoS}_{2}-\mathrm{CdS}$ or NiS-CdS microspheres embedding Te microtubes, which were proven to promote the photoreforming of lactic acid under visible light [227].

5.3.1.2. CdS-based solid solutions. Solid solutions of the $\mathrm{Cd}_{x} \mathrm{Zn}_{1-x} \mathrm{~S}$ general formula have been studied as tuneable band gap semiconductors for photocatalytic $\mathrm{H}_{2}$ generation. Equimolar $\mathrm{Cd} / \mathrm{Zn}$ was found as the optimum composition for glucose as the substrate and $\mathrm{Pt}$ as the co-catalyst [63]. Based on XPS data, surface hydroxylation was suggested for these sulfide solids in basic aqueous media [228]. Different $\mathrm{Cd}_{0.5} \mathrm{Zn}_{0.5} \mathrm{~S}$ structures were synthesised by either hydrothermal methods (hexagonal) or precipitation (cubic); the latter exhibited higher glycerol photoreforming activities using $\mathrm{Pt}$ as the co-catalyst, although higher surface areas might have influenced $\mathrm{H}_{2}$ productivities [64]. In a similar research, a sonochemical synthetic method was applied for the precipitation of 
zinc and cadmium sulfides in the presence of thiosulfate anions; the best performer, which contained significant amounts of $\gamma-\mathrm{Zn}(\mathrm{OH})_{2}$, corresponded to a 0.6:0.4 Cd/Zn atomic ratio [229]. Another report dealing with photocatalytic production of $\mathrm{H}_{2}$ from aqueous ethanol also concluded on $\mathrm{Cd}_{0.6} \mathrm{Zn}_{0.4} \mathrm{~S}-\mathrm{Zn}(\mathrm{OH})_{2}$ composites, having photodeposited platinum nanoparticles as co-catalysts, as the optimum system [230]. In addition to all these systems based on CdS as the main semiconductor, heterojunction systems whereby $\mathrm{CdS}$ absorbs visible light and the photo-generated charges migrate to titania have been described above (Section 5.1.4).

5.3.2. Sulfides other than CdS. 5.3.2.1. Zinc sulfide. Among simple sulfides of metals other than cadmium, zinc sulfide is the most frequently studied. Unmodified $\mathrm{ZnS}$ is only active under UV light $\left(E_{\mathrm{g}}=3.6 \mathrm{eV}\right)$ [41]. Production of $\mathrm{H}_{2}$ from aqueous alcohols (mainly methanol and ethanol) under UV-visible light using bare $\mathrm{ZnS}$ as the photocatalyst material has been reported [97b, 231]. It is worth mentioning that dimeric products, such as ethylene glycol from methanol, were the major oxidation products found in the liquid phases after irradiation [97b, 231a]. Composites based on $\mathrm{ZnS}$ and $\mathrm{Bi}_{2} \mathrm{~S}_{3}$ nanoparticles deposited on $\mathrm{ZnO}$ nanorods (in turn grown on graphene) have been designed as active systems for glycerol photoreforming under light of wide spectrum, owing to the complementary absorption profiles of the different semiconductors [232].

5.3.2.2. Mixed sulfides. Ternary chalcogenides including zinc and other metals may enable charge separation under visible light. For example, $\mathrm{ZnIn}_{2} \mathrm{~S}_{4}$ was found to promote $\mathrm{H}_{2}$ production from aqueous glucose at wavelengths longer than $420 \mathrm{~nm}$ using $\mathrm{ZnS}$ as a coating material and $\mathrm{Pt}$ as the co-catalyst for hydrogen evolution [58]. In another report, $\mathrm{CuInS}_{2}$ (deposited on $\mathrm{TiO}_{2}$ by solvothermal methods) was chosen as the component for absorption of visible light for the ethanol photoreforming reaction [233]. The possibilities to design materials with engineered band gap for photocatalytic production of $\mathrm{H}_{2}$ under visible light can be extended to quaternary chalcogenides, as in the case of $\operatorname{AgInZn}{ }_{7} S_{9}\left(E_{\mathrm{g}}=3.6 \mathrm{eV}\right)$ [234].

5.3.3. Other chalcogenides. In addition to the most frequently employed sulfides, other metal chalcogenides are interesting semiconductor materials with narrow band gaps. The most prominent examples of these are CdSe and CdTe. According to early studies, their activities were limited as compared to $\mathrm{TiO}_{2}$ [214]. Band gap engineering is possible by designing appropriate solid solutions of the $\mathrm{CdS}_{x} \mathrm{Se}_{1-x}$ general formula, as reported by Kawai and co-workers [235]. They found that increasing the selenium-to-sulfur ratio, $\mathrm{H}_{2}$ production from aqueous ethanol under UV-visible light decreased until negligible values for pure CdSe, a fact ascribed to the rise in the valence band energy of the material, which thus becomes less oxidising. In contrast, formic acid, with a less positive redox potential, could be photoreformed regardless of the solid solution composition. 
According to a recent method, surface modification of CdSe nanocrystals with appropriate pendant moieties with attached carboxylate functionalities renders the material water-soluble, and upon addition of nickel [236] or cobalt [237] species to the resulting colloidal solution, such systems exhibit extremely high turnover numbers for $\mathrm{H}_{2}$ production from aqueous ascorbic acid under $520 \mathrm{~nm}$ light irradiation. This approach has also been applied to CdS@CdSe core/shell nanoparticles [238] or CdTe quantum dot systems comprising homogeneous cobalt co-catalysts [239], and in all cases, activities and quantum yields were significantly elevated using small amounts of photocatalytic material.

\subsection{Photocatalysts based on novel semiconductors}

In contrast to the widely investigated chalcogenides, other classical semiconductor classes, such as phosphides, nitrides, carbides, polymers or elemental substances have been seldom studied [240], most likely due to their relative instability in aqueous media [241]. Early work by Sakata and Kawai demonstrated that, based on $\mathrm{H}_{2}$ production under UV-visible light using deposited $\mathrm{Pt}$ as the cocatalyst, activity follows the order: $\mathrm{TiO}_{2}>\mathrm{CdS}>\mathrm{WSe}_{2}>\mathrm{MoS}_{2}>\mathrm{CdSe}>\mathrm{SiC}>\mathrm{CdTe}>\mathrm{Fe}_{3} \mathrm{O}_{4}$ $>\mathrm{Si}>\mathrm{GaAs}>\mathrm{GaP}>\mathrm{Fe}_{2} \mathrm{O}_{3}>\mathrm{InP}>\mathrm{WO}_{3}$ [214]. Rather modest photoactivities and deactivation events have discouraged the further development of semiconductors by simple combination of nonmetal and metal elements. Corrosion of n-type Si during ethanol photoreforming was reported, albeit surface modification with polymeric substances and noble metals ( $\mathrm{Pt}, \mathrm{Ag}$ ) led to certain improvements in performance [100e]. It has not been until very recently that completely new approaches in synthetic methodologies have paved the way towards novel semiconducting materials with appropriate robustness for realistic applications in photocatalysis. The demonstration of nanocarbon materials, polymers, metal-organic frameworks or covalent-organic frameworks as effective light-absorbing components is currently beginning to blossom.

5.4.1. Carbon nanomaterials. In the case of nanocarbon materials, the burgeoning field of graphene and carbon nanotubes has positively inspired photocatalysis researchers [158b, 242], mostly by considering these intrinsically conductive materials (owing to their two-dimensional conjugated $\pi$-system) as electron mediators which may facilitate the mobility of photo-generated charges from the light-absorbing material to appropriate surface active sites. Moreover, the design of semiconducting graphene has been made possible by doping with heteroatoms such as nitrogen [243] or phosphorus [244], thus resulting in genuine metal-free photocatalysts for $\mathrm{H}_{2}$ production from methanol/water mixtures. Under similar conditions, graphene oxide has been proposed as a photo-active semiconducting material, although structural damage and agglomeration was observed after irradiation [245]. Graphitic carbon nitride $\left(g-\mathrm{C}_{3} \mathrm{~N}_{4}\right)$ is a novel semiconductor with undoubted 
possibilities in metal-free catalysis. Its band gap $(2.7 \mathrm{eV})$ allows activation under light of wavelengths below $c a .460 \mathrm{~nm}$. Given these features, application of $g-\mathrm{C}_{3} \mathrm{~N}_{4}$ in photocatalytic fuel generation is a field of research experiencing rapid evolution [246]. This has been proved for the production of $\mathrm{H}_{2}$ under visible irradiation from either water/alcohol mixtures [247] or aqueous ascorbic acid [248]. The role of $g-\mathrm{C}_{3} \mathrm{~N}_{4}$ as a light-absorbing semiconducting material able to activate $\mathrm{H}_{2}$ generation has been demonstrated by comparing its activity with an isolating silica support (SBA-15), which resulted to be inactive under the same conditions, i.e. $\mathrm{MoS}_{2}$ as the cocatalyst and lactic acid as the photoreforming substrate [249]. Carbon nanoparticles, obtained by combustion/pyrolysis of vegetable oils, have also been claimed as active photocatalyst materials when used in combination with photodeposited $\mathrm{Pt}$ for the ethanol photoreforming reaction [250].

An important issue to stress regarding carbon photocatalysts is their stability under prolonged irradiation. In fact, several researchers have noticed progressive deactivation in such cases. For example, $\mathrm{H}_{2}$ production rates were observed to decline during methanol photoreforming using nitrogen-doped graphene, a phenomenon which was ascribed to damage to the material, as evidenced by the observation of dense aggregated particles by TEM [243]. Yet in another report, carbon nitride materials containing pendant amino groups were prone to photo-degradation, as suggested by elemental analyses after irradiation [251]. These findings, together with the known susceptibility of many diverse carbon substances - including coal or hydrocarbons - for being themselves consumed by photoreforming events under appropriate conditions [161], should be kept under careful consideration whenever carbon-containing materials are included in photocatalytic materials, and stability studies combined with thorough characterisation of used catalysts should be performed where possible.

\subsubsection{Metal-organic frameworks (MOFs) and covalent-organic frameworks (COFs).} Semiconducting properties of metal-organic frameworks have been recently demonstrated, as discussed by Corma, García and co-workers [252]. One particular example was experimentally proved in the case of zirconium-benzenedicarboxylate materials, which induced transient charge separation (living for $>300 \mu \mathrm{s}$, based on laser flash photolysis and time-resolved UV-visible emission) upon light absorption, generation of $\mathrm{H}_{2}$ by methanol photoreforming and to exhibit noticeable water stability [253].

An even less mature, albeit promising, class of materials is that of the entirely organic, highly porous and crystalline covalent-organic frameworks. Semiconducting properties have been demonstrated for these novel substances. In a very recent example, $\mathrm{H}_{2}$ generation photoactivity has been demonstrated for a two-dimensional covalent organic framework (composed of aromatic 
triazine and hydrazide units) under visible light [254]. The structure of these materials may, in principle, be modified in multiple ways, and thus, their properties might be tuned accordingly. Therefore, a fruitful field of research can be envisaged for them in photocatalysis.

5.4.3. Polymeric semiconductors. Depending on their structures, linear chain polymers can exhibit semiconducting properties. Nevertheless, their use in photocatalytic materials has raised limited research activity. In a particular report, poly(3-hexylthiophene) has been chosen as lightharvesting component in composites incorporating $g-\mathrm{C}_{3} \mathrm{~N}_{4}$ as electron mediator and $\mathrm{Pt}$ as cocatalyst for the production of $\mathrm{H}_{2}$ from aqueous ascorbic acid under visible light [255]. Owing to its narrow band gap ( $c a .2 \mathrm{eV}$ ), the participation of poly(3-hexylthiophene) in this polymer/polymer heterojunction system enabled outstanding activities (> $300 \mathrm{mmol} \mathrm{g}_{\mathrm{cat}}{ }^{-1} \mathrm{~h}^{-1}$ ). Despite a significant deactivation amounting to $30 \%$ decreased $\mathrm{H}_{2}$ production rates after several days of operation, these results encourage further investigations on such inexpensive carbon-based photocatalysts.

\subsection{Co-catalyst engineering for $\mathrm{H}_{2}$ production}

The efficiency of photoreforming processes, as discussed in the dedicated section (4) about reaction mechanisms, can be greatly enhanced by the incorporation of a $\mathrm{H}_{2}$ evolution co-catalyst on the light-absorbing semiconductor [256]. Surface-deposited metallic or metallic oxide nanoparticles are the preferred co-catalysts in most cases, although recently, sulfide or phosphide counterparts are gaining increasing attention. For decades, noble metals have been the basis of best performers in photocatalytic $\mathrm{H}_{2}$ production, although due to their elevated cost, many researchers are turning their focus towards earth-abundant alternatives [28, 257]. Other investigations have dealt with nanocarbon materials [257a], metal complexes [7a, 256, 257b], metalloenzymes [258] or metalloenzyme mimics [256-257, 259] as the active species for hydrogen evolution. In the following sections, the most relevant aspects of co-catalyst engineering for the photocatalytic production of $\mathrm{H}_{2}$ from biomass-derived oxygenates are summarised.

5.5.1. Metal or metal oxide co-catalysts. As demonstrated by time-resolved transient monitoring (see Section 4.1 above) [45b], metallic (nano)particles deposited on the surface of the semiconductor often serve as traps or collectors for photo-generated electrons and catalyse reduction of protons (or proton-containing molecules) to $\mathrm{H}_{2}$, depending on the work function of the metal and on the potential of the redox processes involved [7d, 260]. The characteristics of the Schottky junction between metal and semiconductor are of paramount importance for this mode of charge transfer. Appropriate synthetic metal deposition methods include impregnation, precipitation or photodeposition. Impregnation involves direct contact of the semiconductor powder with a solution of a salt of the desired metal (or metals) and subsequent evaporation to dryness. The 
impregnated salt is generally further transformed into metal or metal oxide nanoparticles by annealing. Depending on the metal, the occurrence of metal or metal oxide phases can be tuned by annealing atmosphere and temperature. For example, noble metals are readily reduced to their elemental form, whereas most non-noble metals require the presence of hydrogen. Loading metals by precipitation entails the formation of metallic (nano)particles in solution in the presence of suspended semiconductor powders. This can be achieved by (among different routes) pH-induced formation of oxides or chemical reduction, leading to metal oxide or metallic particles, respectively. Finally, photodeposition is a convenient method since it can be performed in situ at the beginning of the photocatalytic experiments [213b, 261]. It is based on the reduction of metallic cations by photo-generated electrons on semiconductor surfaces, thus leading to the growth of closely contacted metallic deposits. A comparative study by Haruta and co-workers stated photodeposition was the most suited metal loading method for the performance of $\mathrm{M} / \mathrm{TiO}_{2}(\mathrm{M}=\mathrm{Au}$ or $\mathrm{Pt})$ in $\mathrm{H}_{2}$ production from aqueous ethanol under UV-vis light [262]. Changes in the oxidation state (generally, reduction) of the metal are frequently observed under irradiation. Many factors related to the co-catalyst, e.g. concentration, dispersion, morphology or oxidation state may influence the outcome of a photocatalytic reaction. The dependence of photoreforming activity on the amount of loaded metal co-catalyst is generally critical. Most often, an optimum concentration might be determined, below which, activity increases with increasing availability of surface metallic sites. Above this point, undermined photocatalysis has been attributed to a variety of factors, e.g. lightblocking, restriction to substrate oxidation on semiconductor surfaces, or electron-hole recombination events on excessive co-catalyst. Bowker and co-workers specifically studied this issue by developing a geometric model whereby light-induced hydrogen production rates from aqueous methanol on $\mathrm{Pd} / \mathrm{TiO}_{2}$ is directly dependent upon the available perimeter around the interface between semiconductor surface and co-catalyst particles [91a, 263]. According to this model, charge transfer reactions involving adsorbed substrate species would take place preferentially near the metal-support interface, which becomes more ubiquitous with increasing metal loading up to a maximum above which particles merge and the perimeter is reduced (Figure 23) [91a, 263].

5.5.1.1. Noble metal co-catalysts. Noble metals are the most widely investigated components of $\mathrm{H}_{2}$ evolution co-catalysts. This is most likely inherited from platinum electrodes as cathodes in electrochemical cells. Although surpassing the activity of Pt is challenging, systems relying on other noble and non-noble metals have also proved efficient for $\mathrm{H}_{2}$ evolution [28, 257]. Given the many experimental variables involved (e.g. type of substrate, illumination source and intensity, $\mathrm{pH}$ or temperature), and to varied features of the metal deposits, an absolute activity scale cannot be 
proposed. However, a number of reports include comparisons of different metal co-catalysts deposited on titania under similar conditions. Investigations by Bowker and co-workers have suggested that methanol photoreforming efficiencies decrease in the $\mathrm{Pd}>\mathrm{Pt}>\mathrm{Ir}>\mathrm{Au}>\mathrm{Ru} \approx \mathrm{Rh}$ $>$ Ni metallic order [264], whilst Silva et al. ranked Pt as the best performer [159a]. Studies on a range of alcohols as substrates, the activity has been ranked following the $\mathrm{Pd}>\mathrm{Pt} \geq \mathrm{Au}$ series, although when sorting the data relative to surface metal atoms, taking into account nanoparticle sizes, the order reverted to $\mathrm{Au}>\mathrm{Pd} \geq \mathrm{Pt}$ [265]. Similarly, $\mathrm{Fu}$ et al. found that $\mathrm{H}_{2}$ production decreased along the $\mathrm{Pd}>\mathrm{Pt}>\mathrm{Au} \approx \mathrm{Rh}>\mathrm{Ag} \approx \mathrm{Ru}$ series for reactions using glucose as the substrate [62a]. For carboxylic acids, $\mathrm{H}_{2}$ production diminished in the following order: $\mathrm{Pt}>\mathrm{Pd}>\mathrm{Rh} \approx \mathrm{Au}$ $>\mathrm{Cu} \approx \mathrm{Ag}$ [37]. In general, according to these and other published data, it appears that Pt is the most effective metallic element, followed by $\mathrm{Pd}$, for photoreforming catalysts. A typical TEM micrograph showing the dispersion of Pt nanoparticles on the titania support is reproduced in Figure 24 [266].

The case of gold is particular, since some early studies reported negligible activity [71], whereas appropriately prepared $\mathrm{Au}$ nanoparticles have proven to become highly active in photocatalysis [267], and in particular for hydrogen evolution by photoreforming [100a, 114, 133, 150, 196b, 262, 268]. Furthermore, gold nanoparticles may perform as sensitisers, due to the visible light absorption enabled by their surface plasmon resonance [186, 269]. Therefore, the uniqueness of $\mathrm{Au}$ nanoparticles resides in combining the suitability of its surface for $\mathrm{H}_{2}$ evolution and the possibility of initiating the photocatalytic process by generating charge carriers under visible light (500$650 \mathrm{~nm}$ ) [267, 269b]. Efficient reduction to metallic gold nanoparticles supported on semiconductor (e.g. $\mathrm{TiO}_{2}$ ) powders can be performed by simply annealing in air at moderate temperatures [115, 133, 270]. The preparation of $\mathrm{Au} / \mathrm{TiO}_{2}$ nanotube arrays by anodisation of titanium-gold alloy sheets and subsequent annealing in air has been reported [270]. The size of Au nanoparticles is an important factor for $\mathrm{H}_{2}$ production, since it affects both the absorption maximum associated to the surface plasmon resonance and the density of available active sites. Higher rates are generally observed for decreasing sizes [122, 269a], although the influence was not relevant in the 3-12 nm range for reactions involving ethanol as the substrate and anatase as the $\mathrm{TiO}_{2}$ phase [114].

5.5.1.2. Non-noble metal co-catalysts. Aiming at developing inexpensive metal co-catalysts, many researchers have rated their activities relative to noble metal benchmarks. Copper [28] and nickel [257b] are the most frequently studied Earth-abundant examples. This reflects their relatively high current production rates: Copper is the second metal in global annual production after iron, whereas both copper and nickel supplies appear to be reasonably scalable [271]. It is relevant to note that the 
suitability of different metals for photoreforming reactions parallels the general trends of dark thermal aqueous phase reforming: $\mathrm{Pt}$ and $\mathrm{Pd}$ are the most suited for $\mathrm{H}_{2}$ selectivity, whereas Ni promotes $\mathrm{C}-\mathrm{C}$ scission reactions and $\mathrm{Cu}$ performs best as water gas shift active metal [17c]. Copper and nickel have been reported to exhibit performances on a par with platinum [95]. It should be stressed that copper oxide deposits on $\mathrm{TiO}_{2}$ often undergo reduction under UV light [272]. Highly efficient titania materials can be prepared by $\mathrm{Cu}$ deposition-precipitation with appropriate loading levels and subsequent annealing in air; above a certain copper content $(<2 \% \mathrm{CuO}$ by weight $)$ whereby the metal deposits change from monolayer to nanoparticles, the system was claimed to be less efficient due to deeper energies of the conduction band of $\mathrm{CuO}$, which thus becomes inefficient for $\mathrm{H}_{2}$ reduction [273]. Spectroscopic techniques have been used to determine the oxidation state as $\mathrm{Cu}^{2+}$ in this and other studies dealing with similar photocatalysts [274]. Conversely, the use of deliberately reduced (by annealing under hydrogen prior to photoreforming) materials of this kind has been also reported and their performances found to be superior than those of oxidised (only annealed under air) analogues [275]. For reduced $\mathrm{Cu} / \mathrm{TiO}_{2}$, partial oxidation of copper was observed after irradiation [275], in contrast to the reduction of titania-supported copper oxide photocatalysts mentioned above. Overall, these indications may imply complex redox process for copper species in these systems, probably reaching steady-states at mixed oxidation states. Copper leaching has been observed in glycerol photoreforming experiments under UV light [276]. Similar issues were observed by Fornasiero and co-workers for photocatalysts prepared by impregnation of copper; to circumvent this, they designed a microemulsion-based synthetic method whereby $\mathrm{CuO}_{x}$ nanoparticles remained embedded within the titania phases and thus protected from acidic reaction intermediates [272a]. Further studies in this direction for titania materials loaded by photodeposited $\mathrm{Cu}$ demonstrated that copper leaching was minor under UV light, but noticeable under solar-like irradiation, although the decrease in activity was not critical, owing to a steady state whereby an equilibrium of $\mathrm{Cu}$ species is reached [277]. Kondarides and co-workers have developed an equilibrium deposition filtration methodology for copper loading leading to highly dispersed $\mathrm{CuO}$ nanoclusters strongly interacting with the $\mathrm{TiO}_{2}$ support, and applied these materials to glycerol photoreforming, observing fluctuations in $\mathrm{H}_{2}$ and $\mathrm{CO}_{2}$ evolution due to light-induced $\mathrm{Cu}$ redox processes $[272 b]$. In a different approach, Centi and co-workers described a gas-phase reactor for successfully photoreforming ethanol on $\mathrm{Cu} / \mathrm{TiO}_{2}$ films [120c].

Nickel has also been proven as an efficient component of co-catalysts for $\mathrm{H}_{2}$ evolution from aqueous ethanol under UV light, exhibiting higher activities than gold for low concentrations of the oxygenated substrate [278]. In a comparative report, ethanol photoreforming activity was observed to decrease in the $\mathrm{Cu}>\mathrm{Ni}>\mathrm{Ag}$ order for in situ photodeposited metals on mesoporous $\mathrm{TiO}_{2}$ [279]. 
A similar trend $(\mathrm{Cu}>\mathrm{Ni}>\mathrm{Co}$, presumably in the form of their divalent oxides) was found for the production of $\mathrm{H}_{2}$ by glycerol photoreforming; slower $\mathrm{CO}$ and $\mathrm{CH}_{4}$ generation, and thus higher $\mathrm{H}_{2}$ selectivity was achieved using the nickel co-catalyst [280]. According to a similar study entailing the use of $\mathrm{BaTi}_{4} \mathrm{O}_{9}$ as the semiconductor, the following $\mathrm{H}_{2}$ production rate order was determined: $\mathrm{Pt}$ $>\mathrm{Co}>\mathrm{Cu}>\mathrm{Ni} \approx \mathrm{Ru}[204]$. The sizes of nickel nanoparticles prepared by a solvothermal method affected the outcome of $\mathrm{H}_{2}$ production using $\mathrm{CdS}$ nanorods as the light absorber under visible light from aqueous lactic acid, with smaller particles accelerating the reaction; furthermore, Ni proved only slightly less efficient than Pt, but noticeably superior than Co or Fe [281].

Cobalt co-catalysts on titania have been less extensively investigated than nickel or copper counterparts, yet their study is worth being considered. Recently, a method based on the hydrolysis of titanium alkoxides in the presence of cobalt salts and subsequent calcination led to segregation of Co in the form of highly dispersed nanoclusters on the external surface of the nascent anatase phases; activity of the resulting material for methanol photoreforming was proven [282]. Deposition of isolated tin species has also proved beneficial to enhance the performance of $\mathrm{Pt} / \mathrm{TiO}_{2}$ photocatalysts [283].

5.5.1.3. Alloy nanoparticles as co-catalysts. Alloy nanoparticles with variable composition have also received attention as tuneable $\mathrm{H}_{2}$ evolution co-catalysts. Bimetallic AuPd nanoparticles, prepared by an impregnation-reduction treatment, on anatase, were proven to be more active than monometallic $\mathrm{Pd}$ or Au counterparts for the light-induced production of $\mathrm{H}_{2}$ from aqueous ethanol [100f, 100g]. Similar alloy materials prepared by impregnation-annealing (without reductive treatment) were studied by XPS, which revealed the presence of $\mathrm{Au}(0), \mathrm{Pd}(\mathrm{II})$ and $\mathrm{Pd}(0)$, whereas Ar sputtering was used to further reduce palladium to its metallic form [284]. Deposition methods using urea were also reported for the preparation of AuPd nanoparticles on $\mathrm{TiO}_{2}$ inverse opals with ethanol photoreforming activity [285]. Synergistic effects in the photocatalytic production of $\mathrm{H}_{2}$ from aqueous methanol by alloying gold and palladium has also been suggested, especially for colloid nanoparticles prepared by chemical reduction [286]. A step further in co-catalyst design was given by Hutchings and co-workers, who discovered the superior performance of $\mathrm{Pd}_{\text {shell }}-\mathrm{Au}_{\text {core }}$ nanoparticles immobilised on $\mathrm{TiO}_{2}$ exhibiting high photoreforming quantum efficiencies as compared to random alloy or $\mathrm{Au}_{\text {shell }}-\mathrm{Pd}_{\text {core }}$ counterparts, using a range of biomass-derived alcohols or saccharides (reaching $\Phi \approx 78 \%$ for glycerol, see micrographs in Figure 25) [287]. Llorca and coworkers systematically studied gold-copper alloys for ethanol photoreforming, and found an optimum composition at a 3:1 $\mathrm{Au} / \mathrm{Cu}$ atomic ratio [288]. Another thorough investigation on Ptcontaining alloy nanoparticles using either $\mathrm{TiO}_{2}$ or $\mathrm{CdS}$ as the semiconducting supports was 
performed by $\mathrm{Hu}$ and $\mathrm{Yu}$. They concluded that $\mathrm{H}_{2}$ production from aqueous lactic acid under visible light can be enhanced, especially for CdS, along the following series: $\mathrm{Pt}_{3} \mathrm{Co}_{2}<\mathrm{Pt}_{3} \mathrm{Au}<\mathrm{Pt}_{3} \mathrm{Ni}<\mathrm{Pt}$ $<\mathrm{Pt}_{3} \mathrm{Cu}$, presumably due to the high conductivity and electron accepting ability of the former [217].

5.5.2. Metal sulfide or other non-oxide co-catalysts. 5.5.2.1. Metal sulfide co-catalysts. The most frequently investigated non-oxide material is $\mathrm{MoS}_{2}$, which has a graphite-like layered structure and exhibits superior photo-stability than other metal sulfides, owing to its relatively covalent characteristics [289]. Its proven efficiency in electrochemical cells for $\mathrm{H}_{2}$ evolution [290] or photooxidation [289] has been demonstrated. Regarding their use in photoreforming reactions, activities of $\mathrm{MoS}_{2} / \mathrm{CdS}$ higher than those of analogous $\mathrm{Pt} / \mathrm{CdS}$ materials have been reported in several cases [226, 249, 291]. The outstanding activity of $\mathrm{MoS}_{2} / \mathrm{CdS}$ for $\mathrm{H}_{2}$ evolution under visible light was recently discovered by $\mathrm{Li}$ and co-workers; in part, this might be ascribed to the compatibility of both sulfides, which results in extremely intimate heterojunctions facilitating charge transfer between them (Figure 26) [291b, 292]. Optimised $\mathrm{MoS}_{2} / \mathrm{CdS}$ photocatalysts, prepared by impregnation of molybdenum on $\mathrm{CdS}$ and subsequent annealing in $\mathrm{H}_{2} \mathrm{~S}$ atmosphere, showed excellent performance using lactic acid as the substrate [292], even better than Pt [291b], and relatively lower $\mathrm{H}_{2}$ production rates for molecules only containing hydroxyl functionalities [292]. The close contact between $\mathrm{MoS}_{2}$ and $\mathrm{CdS}$ can be also generated by ball milling followed by annealing under $\mathrm{Ar}$ atmosphere; in this case, the resulting materials outperformed analogue materials prepared by impregnation or $\mathrm{Pt} / \mathrm{CdS}$, for the production of $\mathrm{H}_{2}$ from aqueous lactic acid under visible irradiation [291a]. Not only CdS, but also a metal-free semiconductor, that is, $g-\mathrm{C}_{3} \mathrm{~N}_{4}$ can be loaded with $\mathrm{MoS}_{2}$ to result in notably enhanced photoreforming activity, which followed the overall $\mathrm{MoS}_{2}>\mathrm{Pt}>\mathrm{WS}_{2}$ order [249]. Another report dealt with the optimisation of $\mathrm{WS}_{2} / \mathrm{CdS}$ photocatalyst, and suggested that the performance of this co-catalyst can also surpass that of Pt in these systems [293]. Deposition of nickel(II) sulfide on CdS by hydrothermal methods yielded visible light photocatalysts for $\mathrm{H}_{2}$ production from aqueous lactic acid [99]. Similarly, $\mathrm{NiS} / g-\mathrm{C}_{3} \mathrm{~N}_{4}$ photocatalysts can be prepared, although their activity and stability in the presence of acidic substrates such as lactic, oxalic or ascorbic acids was modest, partly due to photo-oxidation events leading to formation of elemental sulfur [251]. Another newly developed approach aiming at superior charge separation and stability is the design of composites containing graphene materials as electron mediators between the light-absorbing semiconductor and the $\mathrm{MoS}_{2}$ co-catalyst [294].

5.5.2.2. Metal phosphide co-catalysts. As a newly developed class of co-catalysts (not containing noble metals) for $\mathrm{H}_{2}$ generation in light-activated systems, transition metal phosphide nanoparticles are being considered. For example, metal phosphides can be deposited on CdS by solvothermal 
procedures. Following this method, $\mathrm{Ni}_{2} \mathrm{P}$ and $\mathrm{Co}_{2} \mathrm{P}$ have been loaded on $\mathrm{CdS}$ nanorods, and the resulting composites applied to the production of $\mathrm{H}_{2}$ from lactic [295] or mandelic [296] acids, respectively, under visible light. Thermal reduction by hypophosphite, leading to $\mathrm{CoP}, \mathrm{Ni}_{2} \mathrm{P}$ or $\mathrm{Cu}_{3} \mathrm{P}$ supported on hexagonal CdS, and further use in photoreforming of lactic acid, was reported by the same research group [297]. Molybdenum phosphide (MoP), also suggested as co-catalyst for the photocatalytic production of $\mathrm{H}_{2}$ from aqueous lactic acid, has been claimed to exhibit pseudometallic character, based on XPS and UV-vis absorption spectroscopies [298].

5.5.2.3. Other non-oxide co-catalysts. A related set of novel photocatalysts worth exploring are those including chalcogenides other than sulfides, as for example, metal selenides. In this context, a system based on $\mathrm{Co}_{0.85} \mathrm{Se}$, and including $\mathrm{CdS}$ as the light-harvesting semiconductor, graphene as a mediator, and poly(ethyleneimine) as an in situ reducing agent, has been synthesised by solvothermal methods, and its activity for lactic acid photoreforming (similar to that displayed by $\mathrm{Pt} / \mathrm{CdS}$ ) proved [299].

Another scarcely known group of co-catalysts, is that of the transition metal borides. Among these, nickel boride phases have been supported on CdS and the derived materials used in reaction conditions similar to those described above for the phosphides [300]. The results obtained for transition metal phosphides and borides are promising and widen the portfolio of inexpensive $\mathrm{H}_{2}$ evolution co-catalysts for photoreforming applications. However, and as for any new class of materials, thorough stability tests and mechanistic studies are yet required to assess their validity.

5.5.3. Nanocarbon-based co-catalysts. The discovery of nanostructured forms of carbon displaying unique morphologies and physical properties is currently a field of major research activity. Graphene materials (including graphene oxide, reduced graphene oxide or heteroatomdoped graphene), graphitic carbon nitride, carbon nanotubes or carbon nanocones belong to this class of novel materials. The possibility of these materials to catalyse water (or proton) reduction to $\mathrm{H}_{2}$ is a compelling prospect regarding metal-free photocatalysis [158b, 242]. However, and as discussed in section 5.4.1 above, stability and reusability of any carbon material is (albeit feasible $[160,246 \mathrm{~b}])$ always a matter of concern under long-term irradiation.

Photocatalysis using carbon materials as the genuine component providing $\mathrm{H}_{2}$ evolution sites has been recently investigated by several groups. García and co-workers have focused on the synthesis of graphenes by direct pyrolysis of polysaccharides at high temperatures under inert atmospheres followed by ultrasound-assisted exfoliation. By this procedure, they have produced N-doped [243] and P-doped [244] graphenes from chitosan or alginate in the presence of a phosphate salt, respectively, and proved them as valid metal-free photocatalysts - and also interestingly, in the 
absence of any additional semiconductors - for the production of $\mathrm{H}_{2}$ by methanol photoreforming, although at modest rates. Deactivation was observed after long irradiation for N-doped graphene, a fact which calls for the necessity of thorough stability examination of these systems and characterisation of the used photocatalysts. Graphite oxide has also been claimed as a photocatalyst for $\mathrm{H}_{2}$ generation from water/methanol mixture, although again, aggregation and decomposition of the material under UV-vis irradiation was observed [245]. Coupling graphene with classical semiconductors is a stimulating strategy to prove their suitability as co-catalysts for photocatalytic $\mathrm{H}_{2}$ production. In this context, preparation of $\mathrm{TiO}_{2}$ materials with deposited nanocarbon has been reported. A particular report by Fan et al. dealt with $\mathrm{RGO} / \mathrm{TiO}_{2}$ and $\mathrm{CNT} / \mathrm{TiO}{ }_{2}$ composites $(\mathrm{RGO}=$ reduced graphene oxide, $\mathrm{CNT}=$ carbon nanotube) and demonstrated the photoreforming of different alcohols on such composites; the former proved to be more efficient, whereas hydrothermal methods were more suited as compared to light-assisted or chemical reduction [158a]. Production of $\mathrm{H}_{2}$ from aqueous ethanol under $\mathrm{UV}$-vis light was detected for $\mathrm{RGO} / \mathrm{TiO}_{2}$ prepared by hydrothermal treatment of titanium(IV) alkoxides in the presence of graphite oxide [294d]. Other researchers have reported $\mathrm{H}_{2}$ evolution from aqueous glycerol using materials consisting of $\mathrm{ZnO}$ nanorods grown on RGO [190], $\mathrm{ZnO} / \mathrm{RGO}, \mathrm{ZnS} / \mathrm{ZnO} / \mathrm{RGO}$ or $\mathrm{ZnS}-\mathrm{Bi}_{2} \mathrm{~S}_{3} / \mathrm{ZnO} / \mathrm{RGO}$ composites [232], and from lactic acid/water on RGO/CdS under visible light [301]. Ionothermal methods have also been applied to the synthesis of $\mathrm{RGO} / \mathrm{TiO}_{2}$ composites for the photocatalytic production of $\mathrm{H}_{2}$ from water/ethanol mixtures under UV-vis light [302]. Despite the enormous outlook for structural modification of nanocarbon materials, photo-stability still appears as a serious issue. Regarding their use in photoreforming, post-irradiation characterisation, mechanistic investigations and careful analyses of turnover figures and mass balances are a must for future design.

5.5.4. Molecular metal complexes as co-catalysts and organic sensitisers. The transfer of photo-generated charges from semiconductors to molecular species displaying catalytic activity for water (or proton) reduction is a distinct possibility which may benefit from well-defined mechanisms and ready diffusion of the substrate in nearly homogeneous environments [256-257, 259]. Among these molecular co-catalysts, knowledge of metal complexes can be applied [303]. Metalloproteins, especially hydrogenases as those present in natural photosynthetic systems, are also appealing [259b]. For example, CdTe colloids coupled with nickel-iron hydrogenases have been employed for the light-induced production of $\mathrm{H}_{2}$ from aqueous ascorbate [304]. Other approaches, such as homogeneous systems comprising polyoxometalates and platinum co-catalysts for ethanol photoreforming have been described [305]. Similarly, sensitised rhodium or iridiumruthenium complexes perform as $\mathrm{H}_{2}$ evolution catalysts from ascorbate in aqueous solutions under visible light [306]. The well-known tris(2,2'-bipyridyl) ruthenium(II) sensitiser, coupled with 
platinum colloids, has been claimed as the active species catalysing ascorbate oxidation under irradiation from a Xe lamp [307]. Using the same substrate, remarkably high turnover numbers have been found for molecular nickel catalysts either in entirely homogeneous systems (using an organic dye as sensitiser) [308] or colloidal CdSe [236], both under monochromatic (520 nm) irradiation. Cobalt complexes can be also considered as co-catalysts when coupled with CdTe quantum dots as light-absorbing semiconductors (an example is depicted in Figure 27) [239]. A sophisticated supramolecular approach, consisting in the fabrication of nanoribbon hydrogels composed of an organic chromophore and nickel catalysts, has been recently reported for the photocatalytic production of $\mathrm{H}_{2}$ using ascorbic acid as the substrate [309].

The use of organic sensitisers is a well-known strategy to enhance visible light absorption in sunlight harvesting processes, albeit a commonly encountered difficulty in this context is their limited long-term stability under irradiation. In the field of photocatalysis for $\mathrm{H}_{2}$ generation, organic dyes can effectively promote visible light performance of $\mathrm{TiO}_{2}$ materials. A very recent report on this topic describes the judicious synthesis of durable phenothiazines incorporating robust thiophene linkers and alkyloxy pendant groups, their firm adsorption on $\mathrm{Pt} / \mathrm{TiO}_{2}$ solids, and the remarkable stability (per area unit after three $20 \mathrm{~h}$ cycles) of the resulting dye-sensitised photocatalysts during $\mathrm{H}_{2}$ production from ethanol/water mixtures under UV-vis light [310].

Metal complexes offer clear and precise control over their structures and allow for reliable mechanistic and kinetic investigations to be performed. The high turnover numbers reported for photocatalytic $\mathrm{H}_{2}$ production using such systems are encouraging and may assist the design of extremely active photoreforming systems. However, the scope of biomass-derived substrates can still be widened in this area.

\section{Effect of reaction conditions on photoreforming efficiency}

In the preceding sections, the diversity and complexity of the photoreforming processes resulting in the transformation of a wide range of biomass-derived oxygenated substrates into hydrogen and various oxidised species, has been underlined. Obviously, every substrate behaves differently according to its molecular structure, which governs its reactivity and its adsorption tendency on the active surfaces. This has been detailed regarding mechanistic aspects in Section 4, and specific reaction pathways will be further discussed below (Section 7) for each substrate. In the following sub-sections, the operational parameters (i.e. light intensity, $\mathrm{pH}$, temperature, substrate and catalyst concentration or presence of any additives) exerting more influence are outlined, and their importance illustrated with examples. 
The majority of the research reviewed herein has been performed using powdered photocatalysts dispersed in liquid media. In such experimental set-up, the photocatalyst particles can be viewed as small-scale open-circuit photoelectrochemical systems whereby the photo-generated charges separately migrate to either reduction or oxidation surface sites, without the influence of any external potential bias [107b, 213b, 260]. Photocatalyst suspension systems have become extremely popular due to their simplicity, although immobilised films of the material are inherently more practical due to more convenient separation and more uniform irradiation of the solid material. Photoelectrochemical cells [107b, 213b, 311], consisting of (photo)electrodes separated by a conducting wire, together with membrane photoreactors [312], entail more intricate experimental arrangements, although they bear the advantage of allowing separate gas evolution.

\subsection{Effect of light sources and irradiance}

6.1.1. Light sources. Sunlight is a renewable and essentially inextinguishable source of energy. Therefore, use, transformation and/or storage of solar energy are commonly sought-after goals as alternatives to other renewable or non-renewable contenders. In this regard, researchers worldwide are thinking of photocatalysis as a riveting technological approach to generate (solar) fuels by harvesting irradiation from the Sun. Photoreforming of biomass-derived oxygenates has been proved to proceed readily under natural sunlight with partial solar energy storage [128a, 164a, 272c, 313]. In one of these reports, it was proven that heating caused by sunlight is beneficial regarding $\mathrm{H}_{2}$ production rates (up to $105 \mathrm{mmol} \mathrm{g}_{\mathrm{cat}}{ }^{-1} \mathrm{~h}^{-1}$ ) from aqueous glycerol suspensions of an inexpensive photocatalyst, i.e. $\mathrm{Cu} / \mathrm{TiO}_{2}$, with concomitant temperature increases $\left(93{ }^{\circ} \mathrm{C}\right)[313 \mathrm{~d}]$.

Irradiation from the Sun contains a small proportion of UV light $(<400 \mathrm{~nm}, 3-5 \%$ on an energy basis, albeit the percentage of photons of wavelengths is even lower), whereas the rest belongs mainly to the visible and infrared parts of the spectrum [42]. Infrared radiation consists of low energy photons unlikely to induce electronic transitions in semiconductors, although it may result in vibrational transitions which are usually quenched with heat release. Titanium dioxide, the most frequently employed semiconductor for photocatalytic materials, absorbs light at wavelengths below $c a .400 \mathrm{~nm}\left(E_{\mathrm{g}} \geq 3.2 \mathrm{eV}\right)$ [23]. For this reason, intense research efforts have been usually dedicated to extending the activity of photocatalysts into the visible range (see Sections 5.1.5 and 5.3.1). Frequently, solar simulators are used to test the photocatalytic performance of newly designed materials; this technology is likewise popular for the production of $\mathrm{H}_{2}$ by photoreforming [9, 24, 100a, 100f, 100g, 120c, 124, 178a, 195-196, 243, 269a, 277, 314]. Simulated solar light is generally produced using Xe arc lamps as the light source and filters to adjust the spectrum to the standard AM 1.5 average solar radiation at ground level in temperate zones, at an approximate 
irradiance of $1 \mathrm{~kW} \mathrm{~m}^{-2}$, reported by the American Society for Testing and Materials [42]. Xenon lamps emit over almost continuous wavelengths roughly parallel to the solar spectrum; for that reason, they are often employed as a convenient irradiation source for photocatalytic experiments (for a comparison of the emission spectra of a Xe lamp and a solar simulator, see Figure 28). Often, borosilicate glass labware filtering wavelengths below $c a .320 \mathrm{~nm}$ is used in combination with Xe lamps, whereas cut-off filters (blocking light below 400-430 nm) are generally employed for UVfree irradiations. Another common type of light sources with emission spectra composed of several bands in the UV-visible range is that of $\mathrm{Hg}$ (or $\mathrm{Hg}-\mathrm{Xe}$ ) arc lamps. Due to their strong emission at near-UV frequencies, these lamps have been widely used for titania-based photocatalysis.

6.1.2. Photo-action spectra. The choice of the light source should be in accord with the absorption profile of the semiconductor. In order to ascertain whether a reaction is initiated by absorption of light of a particular material, the most appropriate method is to record the corresponding photo-action spectra, that is, the activity as a function of the incident wavelength. This can be accomplished by determining the quantum efficiency using monochromatic light at various wavelengths. For example, the activity of $\mathrm{TiO}_{2}$ materials only under illumination of photons having energies equal or greater than its band gap $(c a .3 .2 \mathrm{eV})$ has been unambiguously proven for photoreforming reactions [59, 314b]. Analogously, materials designed to initiate charge separation and transfer under visible light can be validated by recording their photo-action spectra at the appropriate wavelengths. This is the case for CdS [154a] or Au nanoparticles [186, 269b], absorbing below $520 \mathrm{~nm}$ or across a broad range centred at 550-560 nm, respectively.

6.1.3. Effect of light intensity. Irradiance—radiant power per surface area—of incident light exerts obvious influence on photocatalytic reactions. Since charge separation in a semiconductor is caused by incident photons, increasing irradiance at an appropriate wavelength generally results in higher reaction rates [243, 315]. However, at high irradiances, higher fractions of the incident radiation are expected to be wasted due to physical phenomena such as scattering or reflection. At lower irradiances and in ideal systems where a major proportion of photons are absorbed by the photocatalyst, linear dependences of reaction rates $v s$. intensity should be observed in the extreme case where either all photons lead to charge transfer, whereas square-root dependences are expected in scenarios where electron-hole recombination dominates; between both situations, intermediate behaviours might be observed [70]. As an example of a reaction undermined by electron-hole recombination, the formation rate of radicals from acetic acid linearly increased with the squareroot of UV-visible light irradiance [68a]. An exception to these trends is the unusual exponential 
relationship for laser irradiations in methanol photoreforming, ascribed to the possibility of biphotonic processes [243].

According to the aforementioned reasoning, quantum efficiencies tend to increase towards limiting zero irradiance, whereby all photons would impinge the photocatalyst surface. Lianos and coworkers proved this for $\mathrm{Pt} / \mathrm{TiO}_{2}$ films - having maximum exposed area and thus minimising light scattering - and also showed that increasing power density of the incident radiation reaches a plateau above an optimum value [316]. Theoretically, the performance of any photoreforming system should be enhanced by increasing irradiance below such threshold. A set of experiments in this line demonstrated that this is the case for relatively low light intensities (up to $50 \mathrm{~mW} \mathrm{~cm}{ }^{-1}$ ) at a range of different temperatures for glycerol photoreforming using $\mathrm{Cu} / \mathrm{TiO}_{2}$ as the photocatalyst [313d].

\subsection{Effect of temperature}

As for any other chemical reaction, photoreforming rates increase with temperature according to the activation energy of the limiting step, as defined by the Arrhenius equation. In a process such as that studied herein, triggered by light absorption, the elementary reaction steps entail charge transfer between the irradiated solid and adsorbed species at the surface active sites. As deduced by timeresolved spectroscopic studies, transient photo-generated charges rapidly evolve (at times spans shorter than $1 \mathrm{~ms}$ after photon absorption), either be it annihilation by recombination, or transfer to appropriate redox pairs $[45 \mathrm{~b}, 51-53,55]$. These processes are notably faster than desorption and diffusion events required to renew the layer of adsorbed products with fresh substrates, and which are expected to be accelerated with temperature according to Arrhenius relationships. Therefore, provided the irradiation flux is intense enough to maintain a significant population of charge carriers in the semiconductor, photoreforming efficiencies experience a temperature dependence associated with desorption of products and diffusion/adsorption of substrates. This was elegantly illustrated and quantified by Hussein and Rudham in their study on dehydrogenation of alcohols on $\mathrm{Pt} / \mathrm{TiO}_{2}$ at varying temperatures and light intensities: Whilst at high irradiances reaction rates increased with temperature according to the Arrhenius equation $(\log (r) \propto 1 / T)$ with activation energies $\left(E_{\mathrm{a}}\right)$ around $20 \mathrm{~kJ} \mathrm{~mol}^{-1}$, at lower intensities activities reached a maximum above a definite temperature, thus displaying a nil activation energy range (Figure 29) [70]. Based on similar results, other authors argued that oxidation of adsorbed substrates or $\mathrm{H}_{2}$ desorption are the rate-limiting steps at lower and higher temperatures, respectively [104]. However, recent kinetic models are consistent with a mechanism where substrate surface coverages, directly related to adsorption- 
desorption phenomena, are the main factors affecting reaction rates in accord with Langmuir-type models (see Section 4.2) [77].

In their work on complete photoreforming of several biomass-derived substrates on powdered $\mathrm{Pt} / \mathrm{TiO}_{2}$ in water, Kondarides and co-workers clearly demonstrated that efficiencies can be enhanced by gently heating the suspensions $[9,24,38 \mathrm{a}, 317]$. For example, $\mathrm{H}_{2}$ production rates could be incremented by almost $50 \%$ when going from 40 to $60{ }^{\circ} \mathrm{C}$, while a less noticeable effect was observed at $80{ }^{\circ} \mathrm{C}[24,317]$. The authors stressed that light-driven reaction steps are not expected to be affected by such a slight temperature increase; rather, adsorption/desorption dynamics, diffusion, "dark" oxidation steps involving photo-generated charges or stabilisation of transient intermediates, all having relatively small activation energies, are more likely to be accelerated by gentle heating [24]. Temperature ranges between 30 and $70{ }^{\circ} \mathrm{C}$ were explored for the photoreforming of ethanol on $\mathrm{Pt} / \mathrm{TiO}_{2}$ confirming this effect $\left(E_{\mathrm{a}}=30.8 \mathrm{~kJ} \mathrm{~mol}^{-1}\right)$ [120a]. Even more pronounced boosts in $\mathrm{H}_{2}$ production rates by increasing from 25 to $90{ }^{\circ} \mathrm{C}$ (7- and 40 -fold when using pristine or copperloaded $\mathrm{TiO}_{2}$ nanotubes, respectively) have been recently reported under UV light (365 nm) using aqueous glycerol as reaction media [313d]. Photocatalytic decomposition of acetic acid on $\mathrm{Pt} / \mathrm{TiO}_{2}$ materials was also proven to be favoured by slight temperature increases [89a]. The production of $\mathrm{H}_{2}$ and radicals (which subsequently coupled together) from ethanol and methanol in aqueous $\mathrm{ZnS}$ suspension irradiated with UV-visible light also increased upon heating from 30 to $50{ }^{\circ} \mathrm{C}$ [231a]. As explored for the aqueous glycerol photoreforming using $\mathrm{ZnS} / \mathrm{ZnO}$ nanorods, $\mathrm{H}_{2}$ production rates almost doubled by increasing the temperature from 40 to $80{ }^{\circ} \mathrm{C}$ [191b], or as described in a further study, also from 5 to $25^{\circ} \mathrm{C}$ [191a].

Gas-phase approaches allow wider temperature ranges to be considered. According to a recent report, photoreforming reactions under simulated solar light using a vapour phase methanol/water feed and a $\mathrm{Pt} / \mathrm{TiO}_{2}$ photocatalyst carried out at increasing temperatures $\left(150-300{ }^{\circ} \mathrm{C}\right)$ resulted in drastic increases in $\mathrm{H}_{2}$ production rates [181]. Dark experiments demonstrated that both photocatalytic and thermocatalytic processes took place simultaneously, albeit the former proved significantly more efficient. The authors claimed that the photocatalytic process itself can be thermally accelerated by enhancing oxidation kinetics.

Based on the enhancement of photoreforming upon increasing temperature, dual harvesting of solar irradiation by both genuine light activation of the semiconductor and local heating is a phenomenon exerting obvious benefits on $\mathrm{H}_{2}$ generation. Suitably designed reactors and irradiation configuration, including solar concentrators [318], are likely to be of paramount importance in optimising the efficiency of the photocatalytic processes under consideration. 


\subsection{Effect of $\mathbf{p H}$}

The acidity or basicity of aqueous media may affect the outcome of a photocatalytic process in various ways. This is due to the interplay of several physicochemical phenomena influenced by $\mathrm{pH}$, including band energy positions of the semiconductor, the surface charge and electrokinetic potential (which can be measured as zeta potential) of the photocatalyst surface, speciation of the substrate, adsorption or chemisorption processes, redox potentials of both substrate molecules and protons, stabilisation of either reaction intermediates or products, or particle aggregation [108b, 262, 268c]. Given the complexity of $\mathrm{pH}$ influence and the many photocatalyst/substrate combinations possible, trends or models are unlikely to be of general utility. Herein, an account is presented on the dependence of photoreforming efficiency upon $\mathrm{pH}$, outlining the most plausible reasoning where appropriate.

The bulk structure of a semiconductor is not expected to change significantly upon $\mathrm{pH}$ variations, although surface phenomena and charge transfer events are prone to become affected by the nature of the interface. In this context, band energy positions may change with $\mathrm{pH}$. According to electrochemical measurements, a cathodic shift in the conduction band (and consequently, also in the valence band) potential of $\mathrm{CdS}$ materials at increasing $\mathrm{pH}$ has been suggested and claimed to be the cause of higher ethanol photoreforming rates; the slope of the shift appeared to be lower than the Nernstian dependence of the water oxidation potential (see Figure 30), a fact which would lead to favourable $[\mathrm{OH}]^{\cdot}$ formation and thus, unleash further reaction with the substrate [319]. On the other hand, the valence band position of $\mathrm{TiO}_{2}$ is known to follow standard Nernstian behaviour, parallel to that of water oxidation and reduction, i.e. the changes are equal and the thermodynamics of charge transfer between semiconductor and solution should not be significantly affected [5d, 81$]$. Tsubomura and co-workers determined the flat-band potential of $\mathrm{CdS}$ at different $\mathrm{pH}$ values and found that it remained constant and thus more cathodic under acidic regimes, a fact which would result in favoured proton reduction [218]. However, they observed different $\mathrm{H}_{2}$ production trends on illuminated $\mathrm{Pt} / \mathrm{CdS}$ photocatalysts depending on the substrate: formic acid was more rapidly reformed at acidic $\mathrm{pH}$ - in line with the above hypothesis — and negligible activity was observed at $\mathrm{pH}>13$, probably due to impaired adsorption of formate on the negatively charged surfaces; surprisingly, alcohol photoreforming efficiencies were almost zero in neutral or acidic solutions despite the more cathodic character of CdS [218].

Narrower $\mathrm{pH}$ range $(<2)$ was determined to be suitable for the photocatalytic production of $\mathrm{H}_{2}$ from formic acid on $\mathrm{Cu} / \mathrm{TiO}_{2}$, although the cause might be related to co-catalyst changes [320]. Decrease in $\mathrm{H}_{2}$ production by photoreforming of oxalic acid upon increasing basicity, ascribed as in the case 
above to inhibited adsorption onto negatively charged semiconductor surfaces, has been reported by other researchers [60]. Similar behaviour was reported for feeds rich in acidic substrates using $\mathrm{Pt} / \mathrm{TiO}_{2}$ [321] as the photocatalyst, such as phenolic species in olive mill wastewater [321], or for lactic acid on Ni/CdS [281]. Acetic acid and longer chain analogues may undergo reactions under a wider and more complex scope of pathways. For example, acetic acid decomposition on $\mathrm{Pt} / \mathrm{TiO}_{2}$ is faster under acidic conditions [37, 61, 89], in agreement with the less favourable repulsion with the titania surfaces, yet the activity was significant at $\mathrm{pH}$ values as high as 13 , with higher selectivities for photoreforming (lower $\mathrm{CH}_{4}$ formation via photo-Kolbe process) probably due to more efficient formation of $[\mathrm{OH}]^{*}$ in an environment rich in $[\mathrm{OH}]^{-}$under intense UV-visible irradiation (Figure $31)[37,216]$. The more limited availability (or reducibility) of protons at high $\mathrm{pH}$ has been also suggested as a possible cause to the drop of photocatalytic $\mathrm{H}_{2}$ production activity $[102,219,239 \mathrm{~b}$, $322]$.

Several other investigations have revealed enhancement in $\mathrm{H}_{2}$ production on titania photocatalysts for substrates not containing acidic functionalities (and thus not prone to deprotonation) by increasing $\mathrm{pH}[9,24,36,58,108 \mathrm{~b}]$. Although the reasons have been seldom ascertained, consensus about the oxidation of ubiquitous hydroxyl anion to the corresponding radical is claimed in several reports $[37,108 \mathrm{~b}, 323]$. Other researchers point to possible tendency of irradiated $\mathrm{TiO}_{2}$ to corrode in acidic media [324], or better adsorption of substrates on the photocatalyst under basic conditions $[58,64,325]$. A particular example of this is a system based on $\mathrm{Cd}_{0.5} \mathrm{Zn}_{0.5} \mathrm{~S}$ as the semiconductor and glycerol as the biomass derivative [64]. Contradiction to these results was reported by Silva and co-workers for a similar system, although using $\mathrm{Pt} / \mathrm{CdS}$ as the co-catalyst and an aqueous medium of high ionic strength [223b]. The $\mathrm{pH}$ of the solution is susceptible to experience changes during irradiation, as for the photoreforming of some biomass-derived substrates such as alcohols, which produce carboxylic acids and ultimately $\mathrm{CO}_{2}$, and thus, lead to $\mathrm{pH}$ decreases [262]. It should be noted that any $\mathrm{CO}_{2}$ formed under basic $\mathrm{pH}$ tends to remain in solution $[36,108 \mathrm{~b}]$.

Neutral conditions have been proven advantageous in several studies on the photoreforming of biomass-derived substrates, as for example, for the use of cellulose or lignocellulosic feedstocks [313b]. Occasionally, the point of zero charge for $\mathrm{TiO}_{2}(\mathrm{pH} \approx 6)$ has been claimed as the optimum situation for enhanced adsorption, and thus, increased $\mathrm{H}_{2}$ evolution rates. Behaviours of this kind have been reported for alcohols as the substrates when using titania photocatalysts [94a, 140b, 262, 268c, 280, 326], whereas the opposite pattern has been reported for CdS analogues [213a]. Probably, adsorption events are favoured on the likewise neutral titania surface. In the case of ethanol photoreforming on $\mathrm{Cu} / \mathrm{TiO}_{2}$ materials, the optimum was observed to be at $\mathrm{pH}=10$, in part 
due to the susceptibility of copper to leach out of the catalyst under more acidic media [327]. A similar hypothesis was postulated for nickel on $\mathrm{CdS}$ at $\mathrm{pH}<3$ [281].

As mentioned above, the impact of $\mathrm{pH}$ variations on the photocatalytic $\mathrm{H}_{2}$ production efficiency, and to a more general extent on the complete reforming, might operate by the sum of many different phenomena, and postulating general trends is a troublesome task. Dramatic effects are evident for almost all the systems outlined in this section, and thus, it is clear that $\mathrm{pH}$ is a decisive factor to take into account. Criteria can be proposed regarding some of the physicochemical events affected by $\mathrm{pH}$, mostly speciation and related adsorption, or potential of both substrate redox pairs and semiconductor valence/conduction bands. Regarding the former phenomena, it appears that carboxylic acids tend to be less readily photoreformed under strongly basic conditions, due to the unfavourable adsorption of anionic carboxylate forms. On the other hand, $\mathrm{H}_{2}$ production from nonacidic substrates such as alcohols is generally more efficient at neutral or slightly basic $\mathrm{pH}$. Another important factor to ponder is the stability of any particular photocatalytic material under irradiation at deliberately modified $\mathrm{pH}$ and/or ionic strength of the solution.

\subsection{Effect of substrate concentration}

Transformation of oxygenated substances by photoreforming generally requires the participation of water as the oxidation reagent for stoichiometric production of $\mathrm{H}_{2}$. Therefore, the water/substrate ratio is a factor of vital importance for the selectivity of the process. Individual steps in overall reforming may proceed without the involvement of water, as is the case for alcohol dehydrogenation or formic acid decomposition. Notwithstanding this, the presence of water at the photocatalyst surface may always influence reaction kinetics by acting as an oxidising species forming of intermediate hydroxyl radicals (see Section 4.3.2) or as a suitable medium for proton transport [77, 328]. In the following paragraphs, the effect of substrate concentration, or in other words, its ratio relative to water, on $\mathrm{H}_{2}$ production rates and selectivities is summarised.

Irradiation of $\mathrm{M} / \mathrm{TiO}_{2}(\mathrm{M}=$ noble metal $)$ suspensions in pure water under $\mathrm{UV}$-visible light results in low $\mathrm{H}_{2}$ production rates, whereas addition of little amounts of oxygenated substrates, such as hydroxylated molecules, causes dramatic increases in its evolution, as expected owing to their propensity towards oxidation [98, 154a, 268c]. In the absence of water, the production of $\mathrm{H}_{2}$ is limited to some extent, yet significant and selective, as in the case of sunlight-driven ethanol photoreforming [100a]. Yasuda and co-workers have demonstrated that any oxygenated substrate can be completely photoreformed at (decreasing) limiting zero concentration [39]. In line with this, Taboada et al. studied the vapour-phase reaction of ethanol/water mixtures at ratios between 0 and 100 on $\mathrm{Au} / \mathrm{TiO}_{2}$ under UV light; they observed that photoreforming to $\mathrm{H}_{2}$ and $\mathrm{CO}_{2}$ was favoured at 
low ethanol concentrations, whereas dehydrogenation producing $\mathrm{H}_{2}$ and acetaldehyde proceeded preferentially at ethanol-rich regimes [329]. Comparable but more complex tendencies were found in the liquid phase using Pt as the co-catalyst [330]. In contrast, activities have been reported to decrease drastically for methanol-rich vapours as feedstocks [77, 328]. Similar trends were outlined for aqueous glycerol photoreforming on titania catalysts modified with surface copper oxides [272b]. An optimum concentration of substrate might be found for the majority of photoreforming systems, as for example regarding the production of $\mathrm{H}_{2}$ from aqueous ethanol under UV light on $\mathrm{Pt} / \mathrm{TiO}_{2}$ ( $c$ a. $80 \%$ by volume, Figure 32) [98, 330], on $\mathrm{M} / \mathrm{TiO}_{2}(\mathrm{M}=\mathrm{Cu}$ [327] or $\mathrm{Ni}$ [278]), methanol on $\mathrm{Pt} / \mathrm{TiO}_{2}$ [330], $\mathrm{CuO} / \mathrm{TiO}_{2}$ [272d], $\mathrm{Au} / \mathrm{TiO}_{2}$ nanotube arrays [270] or $\mathrm{MOF}$ photocatalysts [253], or glycerol on $\mathrm{M} / \mathrm{TiO}_{2}(\mathrm{M}=$ noble metal) [331]. In the case of glycerol and using $\mathrm{Pt} / \mathrm{TiO}_{2}$ as the photocatalyst, drastic decreases were observed above $50 \%$ by volume, probably due to the strong adsorption of the polyhydroxylated substrate [330, 332]. The pattern for Pt/CdS under visible light irradiation is comparable, except for ethanol rich regimes, which proved detrimental for activity [213a].

In aqueous solutions of low to medium substrate concentrations, the photoreforming efficiencies usually follow a Langmuir-Hinshelwood kinetic model, as discussed in Section 4.2.1 [58, 62a]. At this point, temperature increases would exert a further accelerating effect (see Section 6.2) [201a]. Reported examples of the Langmuir-Hinshelwood behaviour include acetic acid decomposition on Fe-modified $\mathrm{TiO}_{2}$ [333], production of $\mathrm{H}_{2}$ from methanol [43, 140b], glycerol [24, 334], cellulose [313b], sucrose [62b] or olive mill wastewater [321] on $\mathrm{Pt} / \mathrm{TiO}_{2}$, from methanol [272d] or glycerol [272b, 272c] on $\mathrm{CuO} / \mathrm{TiO}_{2}$, from methanol on $\mathrm{RGO} / \mathrm{TiO}_{2}$ [158a], and from glycerol on $\mathrm{Pt} / \mathrm{Cd}_{0.5} \mathrm{Zn}_{0.5} \mathrm{~S}$ [64].

The effect of water/substrate ratios is obvious from the many reports dealing with this, either in liquid or vapour reaction media. Selli and co-workers studied this for vapour-phase methanol photoreforming; water played simultaneous roles by performing as a hydroxyl radical source via its oxidation by holes, and as a proton-conduction medium ensuring transport to reduction sites (see Section 4.3.2) [77, 328]. In aqueous media, water supply to photocatalyst surfaces is guaranteed, whereas in the gas phase, diffusion limitations are minimised [43, 100c, 103, 268b]. Substrate availability is also a requirement for efficient photoreforming, whilst too high concentrations prove deleterious for activities and selectivities to complete reforming (and thus, to $\mathrm{H}_{2}$ ). Thus, optimum systems are those where water/substrate ratios fall within intermediate regimes.

\subsection{Effect of catalyst concentration}


As for any photocatalytic process, reaction rates of aqueous phase photoreforming are directly dependent upon the available amount of light-harvesting material, and likewise, on the amount of incident photons. Quantum yields are expected to reach maximum values at irradiances and catalyst concentrations such that the largest proportion of photons are efficiently absorbed, i.e. such that light scattering, transmission and reflection are minimised. Pichat and co-workers proved that this was the case by measuring production rates of $\mathrm{H}_{2}$ and light intensity for variable masses of $\mathrm{Pt} / \mathrm{TiO}_{2}$ photocatalyst in the course of methanol photoreforming experiments; after a linear dependence region, maximum efficiencies corresponded to total photon absorption [43]. Compatible results have been independently reported, in occasions concluding that the optimum catalyst concentration lies around $2 \mathrm{~g} \mathrm{~L}^{-1}$ [108d, 140b, 313b, 325]. In the case of $\mathrm{ZnS} / \mathrm{ZnO}$ nanorods arrays for glycerol photoreforming, however, the optimum was $1 \mathrm{~g} \mathrm{~L}^{-1}$ [191a]. Other investigations, considering mass transfer effects in the desirable turbulent regimes for actual solar photoreactors, have suggested lower catalyst concentrations in the order of $10^{-1} \mathrm{~g} \mathrm{~L}^{-1}$ for more efficient operation [335].

Obviously, the most appropriate catalyst concentration for suspensions depends on the lightabsorbing features and aggregation behaviour of the powder. An important point to note in this context relates to the form of expressing the photocatalytic activity. When reported on a per mass basis (e.g. moles of $\mathrm{H}_{2}$ per gram of catalyst per hour), the derived data may depend on the catalyst amount, especially for concentrations above the optimum. Therefore, using limited amounts of photocatalyst usually proves advantageous in this regard [336], although comparing data weighted by mass might be misleading and does not guarantee that a maximum $\mathrm{H}_{2}$ production rate is attained in absolute terms. In optimising photoreforming reactions, assessment based also on total amount of evolved $\mathrm{H}_{2}$ is desirable. These examinations are especially relevant for novel photocatalytic systems, for which little information is available [243].

\section{Biomass-derived substrates for hydrogen production by photoreforming}

Hydrogen fuel can be obtained by light-activated reforming of biomass-derived substrates at high efficiencies and using the Sun as the only energy source. This process outperforms thermocatalytic analogues in terms of selectivity — whilst attaining similar rates — due to the considerably lower (ambient or near-ambient) temperatures of operation at which degradation is limited [25]. As compared to the more desirable overall water splitting, photoreforming of oxygenated substrates has been proven at significantly higher rates and longer-term stability. Part of the incident sunlight energy can be stored in the final $\mathrm{H}_{2}$ fuel. Given these arguments, the production of $\mathrm{H}_{2}$ from biomass 
appears as a worthwhile technology for both valorisation of biomass streams in biorefineries and harvesting of solar energy.

Critical assessments are strict requirements to dictate whether specific biomass-derived substrates are suitable for $\mathrm{H}_{2}$ production. In this regard, the energy efficiency of the overall sum of processes, starting from raw biomass and concluding with hydrogen, is a parameter of paramount importance. A significant body of published work on the development of photocatalysts for $\mathrm{H}_{2}$ production has been performed using pure oxygenated substances. From the perspective of efficient biomass-tofuels technologies, research devoted to the use of the rather complex raw feedstocks, such as primary biorefinery products (wood, sawdust, rice husks, etc.), is of vital relevance. Agricultural, industrial or communal waste streams are interesting candidates given the possibility for both treatment/disposal and valorisation via $\mathrm{H}_{2}$ production. In this section, the use of pure substances with potential to be produced from biomass, including alcohols, polyols, aldehydes, ketones, carboxylic acids or saccharides is reviewed and critically evaluated. Photoreforming $\mathrm{H}_{2}$ production rate data have been collected from the literature, transformed to consistent units ( $\mu$ mol $\mathrm{g}_{\mathrm{cat}}{ }^{-1} \mathrm{~h}^{-1}$ ), and summarised in consolidated tables (Table 2-Table 8) for convenient comparative purposes, especially with regard to the different classes of photocatalytic materials employed. It should be noted that reported activity data have been produced under extremely varied reaction conditions (i.e. light intensity, catalyst and substrate concentrations, temperature, $\mathrm{pH}$ ). Yet, the summary of $\mathrm{H}_{2}$ yields presented herein provides a clear and informative report and allows comparisons to be made between different systems.

\subsection{Photoreforming of alcohols}

Although alcohols are not major components of raw biomass, a range of hydroxylated or polyhydroxylated substances can be found in processed streams. The main example is glycerol, which is a multi-ton by-product of the biodiesel (fatty acid methyl esters) industry [10, 337]. Several simple alcohols, being ethanol the most common, can be obtained by fermentation of saccharides [1a, 1d]. Complex pyrolysis biofuels may also contain variable amounts of methanol, ethanol and other alcohols [19-20]. In addition to photoreforming processes, selective photocatalytic oxidations of alcohols either in the presence or absence of oxidising agents can enrich the toolbox of green organic synthetic protocols [31, 338].

7.1.1. Methanol. Currently, methanol production largely relies on the catalytic transformation of synthesis gas, which is in turn obtained from fossil hydrocarbons by steam reforming [339]. Although biomass is also a viable source of synthesis gas by steam reforming or gasification [1a, 
339], the related processes are energy intensive. The practical and economical utility of the biomass $\rightarrow$ syngas $\rightarrow$ methanol $\rightarrow$ hydrogen route appears questionable. Methanol can be also present in the complex mixtures of oxygenated substances produced by direct pyrolysis of raw lignocelluloses, although in low amounts (see Table 1). However, the development of technologies to obtain hydrogen from methanol at low energy expenditure might be worthwhile when considering this alcohol as a hydrogen carrier for feeding fuel cells [340].

The use of water/methanol mixtures for the light-induced production of $\mathrm{H}_{2}$ has been extensively applied in the field, given the availability of the substrate and the high efficiency of the process on a wide variety of photocatalysts. A number of literature examples on the design of photocatalytic materials for $\mathrm{H}_{2}$ production rely on methanol as a substrate. In many cases, these reports have focused on $\mathrm{H}_{2}$ production rates, thus providing valuable information on the suitability of cocatalysts for proton (or water) reduction. In this regard, comparative studies have allowed elucidating the performance on different metal nanoparticles and drawing activity rankings, as that for noble metals $\left(\mathrm{Pt}>\mathrm{Rh}>\mathrm{Pd}>\mathrm{Ru} \gg \mathrm{Ag}\right.$ ) in a particular example using $\mathrm{TiO}_{2}$ as the lightharvesting semiconductor [108d]. In the absence of metal co-catalysts, reforming products have also been detected, although at substantially lower rates and with concomitant reduction of titanium [47a]. Worth noting is the exceptionally high $\mathrm{H}_{2}$ production rates from methanol photoreforming

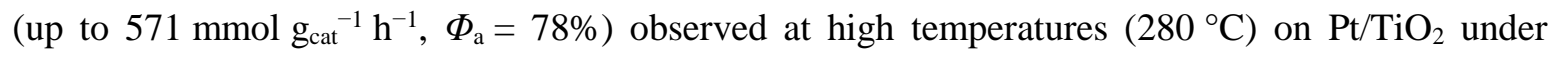
simulated solar light [181]. The fate of methanol, that is, the formation of products by oxidation half-reactions, their production rates and selectivities, has been scarcely investigated in research works aiming mostly at intensifying $\mathrm{H}_{2}$ production. A comprehensive summary of the numerous experimental results on photocatalytic $\mathrm{H}_{2}$ production from aqueous methanol is out of the scope of this review. Rather, selected data covering different classes of photocatalysts and, especially, quantification of oxidation products, are listed in Table 2.

As expected from the photoreforming pathway (see Figure 18 for the generic route), $\mathrm{H}_{2}$ is produced on all occasions by the reduction of protons. In neat methanol, these protons obviously stem from the hydrogen contained in the substrate, whereas in aqueous media, exchange between water and the alcohol takes place to some extent and the $\mathrm{H}_{2}$ produced may (unsurprisingly) originate on both substances. Isotopic labelling experiments have confirmed these hypotheses. A patent fact in this regard is that $\mathrm{D}_{2}$ is mainly produced when using a diluted solution of $\mathrm{CH}_{3} \mathrm{OH}$ in $\mathrm{D}_{2} \mathrm{O}$, although a minor but noticeable amount of HD is also detected due to protonic exchange [38b, 77]. In principle, this phenomenon ought to be common to any substance containing hydroxyl groups. The maximum amounts of hydrogen gas generated do not generally exceed those predicted by the 
stoichiometry of the reforming reaction $[9,38]$. By using $\mathrm{Au} / \mathrm{TiO}_{2}$ photocatalysts and small amounts of methanol, overall water splitting has been claimed [133]. Later, in-depth investigations on similar long-term irradiations of $\mathrm{Pt} / \mathrm{TiO}_{2}$ suspensions in aqueous methanol resulted in production of $\mathrm{H}_{2}$ and $\mathrm{CO}_{2}$ as the sole gaseous products [38b]. In general terms, the main reaction pathway for reactions of methanol/water mixtures under UV-visible light on titania photocatalysts appears to be that represented by the reforming sequence (Figure 18) whereby one mole of methanol gives rise to three moles of $\mathrm{H}_{2}$ and one mole of $\mathrm{CO}_{2}$ (equation 5).

Thorough analyses of methanol photoreforming products have been undertaken on several instances. In addition to $\mathrm{H}_{2}$ and $\mathrm{CO}_{2}$, the main substances formed are formaldehyde and formic acid. Both products tend to remain in the aqueous phase for reactions performed on liquid suspensions [38b], and may undergo further reforming (oxidation) toward eventual $\mathrm{CO}_{2}$ evolution. In the first of these investigations, performed on different $\mathrm{M} / \mathrm{TiO}_{2}(\mathrm{M}=$ noble metal $)$ and under UVrich light the authors detected considerable amounts of $\mathrm{CO}$ and traces of $\mathrm{CH}_{4}$ among the products [328]. In a subsequent work carried out using a light source emitting less UV and more visible light (iron halide-mercury lamp), only traces of CO were detected [77]. It is likely that the origin of CO be photolytic reactions on formaldehyde (dehydrogenation) or formic acid (dehydration). Li and coworkers observed that small amounts of inorganic anions $\left(\left[\mathrm{SO}_{4}\right]^{2-}\right.$ or $\left.\left[\mathrm{H}_{2} \mathrm{PO}_{4}\right]^{-}\right)$inhibited $\mathrm{CO}$ formation from methanol under UV-visible light (Xe lamp) by adsorption on Pt defect sites, which were thus thought to be responsible for formic acid dehydration.

A significant degree of consensus among different literature sources is apparent about the reaction sequence. As illustrated in Figure 33, methanol is first dehydrogenated to formaldehyde, which can be in turn oxidised to formic acid by action of photo-generated holes or hydroxyl radicals, whereas final decarboxylation results in evolution of $\mathrm{H}_{2}$ and $\mathrm{CO}_{2}$, and thus, in complete mineralisation [77]. Accumulation of formaldehyde at short reaction times, a fact which supports dehydrogenation as the first step, has been reported [76]. The transformation of formaldehyde into formic acid has been also postulated as consisting of two steps, namely hydration to methanediol and subsequent lightinduced oxidative dehydrogenation [76]. Further reforming of formaldehyde (and of formic acid) might proceed at comparable or faster rates than the initial dehydrogenation. In such a case, only $\mathrm{H}_{2}$ and $\mathrm{CO}_{2}$ would be detected as products, as reported by Kondarides and co-workers for $\mathrm{Pt} / \mathrm{TiO}_{2}$ photocatalysts operating under UV-vis light and flowing argon [38a]. Another report by the same group described the formation of methanediol, formaldehyde and formic acid, yet as adsorbed species which did not undergo release into the liquid phase [38c]. Bowker and co-workers anticipated such behaviour and stated that formaldehyde and methanol reacted at similar rates on 
$\mathrm{Pd} / \mathrm{TiO}_{2}$, whilst formic acid decomposed even in the absence of light; the authors claimed that build-up of reaction intermediates is unlikely to happen [57]. Instead, a mechanism implying adsorbed CO (see Figure 15) as the main transient intermediate was postulated [91a, 263]. It is reasonable to presume that the distribution of products at steady state for a given photocatalyst depends upon the experimental irradiation set-up. From a mechanistic perspective, the study of methanol photoreforming is a valuable endeavour, not only regarding the transformation of the substrate itself, but also taking into account several reaction pathways starting from higher oxygenates (e.g. ethanol, acetic acid or lactic acid) may entail transient methanol formation.

7.1.2. Ethanol. Biomass is today a major source of (bio-)ethanol through fermentation of saccharides, which can in turn be obtained from lignocellulosic feedstocks via either enzymatic or acidic hydrolysis [1a, 1c, 1d, 341]. The use of bio-ethanol as a fuel itself or as a fuel additive for internal combustion engines is a mature technology. As in the case of methanol, ethanol also occurs in bio-oils generated by direct pyrolysis of raw lignocelluloses, although at low concentrations (see Table 1) [19-20]. Regarding future developments on the implementation of fuel cells for transportation and power generation, ethanol has been also considered as a viable $\mathrm{H}_{2}$ carrier. The transformation can be achieved by thermocatalytic steam reforming [34a, 34b, 35], albeit high temperatures $\left(300-750^{\circ} \mathrm{C}\right)$ and excess steam are generally required to achieve low $\mathrm{CO}$ selectivities and limited carbon deposition on the catalysts. As rationalised in Section 2.3, photocatalytic reforming of ethanol may provide a compelling alternative to this [340], given that it can be promoted by renewable sunlight and proceed at temperatures close to ambient with low extent of degradation events and immeasurable $\mathrm{CO}$ formation.

Ethanol has been chosen as a valid substrate for the photocatalytic generation of $\mathrm{H}_{2}$ using a large number of light-responsive materials. Data from the abundant prior art dealing with photocatalytic $\mathrm{H}_{2}$ generation using ethanol are listed in Table 3. A diverse portfolio of photocatalysts has been deployed by the many research teams devoted to materials design for such a purpose. Hydrogen production rates fall within a wide range. A general inspection of the data in Table 3 suggests that activities increase across different classes of semiconductors in the following order: sulfides < mixed oxides $<$ titanium dioxide. For the latter, $\mathrm{H}_{2}$ production rates may readily surpass $10 \mathrm{mmol} \mathrm{gat}^{-1} \mathrm{~h}^{-1}$. This approximate series is valid for systems based on suspensions of photocatalyst powders. On a different approach, higher productivities have been achieved using nanostructured oxide layers grown by chemical vapour deposition, reaching values in the order of $10^{2} \mathrm{mmol} \mathrm{g}_{\text {cat }}{ }^{-1} \mathrm{~h}^{-1}$ [196a, 197]. 
On the oxidative side, the identity of ethanol photoreforming products as reported across different data sources in the literature (see Table 3 ) is as expected from the generic reaction pathway depicted in Figure 18. In a first step, dehydrogenation promptly proceeds in a two-electron transfer process eventually leading to the formation of acetaldehyde. Quantification at early stages during irradiations of $\mathrm{Au} / \mathrm{TiO}_{2}$ suspensions in liquid ethanol has shown that $\mathrm{H}_{2}$ and acetaldehyde can be produced in essentially equimolar amounts, according to the expected stoichiometry, along with minor amounts of $\mathrm{CO}_{2}$ and other gaseous products [100a]. Whilst gaseous $\mathrm{H}_{2}$ is evolved, acetaldehyde tends to remain in the liquid and partly react with ethanol to yield 1-ethoxyethanol (that is, the corresponding hemiacetal form), as observed by ${ }^{1} \mathrm{H}$ NMR. Other reports have noted the formation of the diethoxy acetal [100g, 277]. Regarding stoichiometry, formation of nearly equimolar amounts of $\mathrm{H}_{2}$ and acetaldehyde has also been reported under similar conditions using $\mathrm{Pt} / \mathrm{TiO}_{2}$ [43], or from ethanol vapour in continuous flow reactors on $\mathrm{Au}_{3} \mathrm{Cu} / \mathrm{TiO}_{2}$ under $\mathrm{UV}$ light [288]. In this latter report and another companion publication [329], it was proven that the presence of water influenced the reactivity pathway towards decreased acetaldehyde and increased $\mathrm{CO}_{2}$ formation (Figure 34). In other words, the first dehydrogenation step toward acetaldehyde is favoured in pure ethanol, whereas the subsequent photoreforming steps proceed more extensively by action of water. Consistent with these findings and argumentations, it is not rare to encounter reports describing intermediate situations whereby $\mathrm{H}_{2}$ is the major product - as the main reduction product - and a range of oxidised species are co-produced [120c, 262, 268b]. Traces of acetic acid have been detected on several occasions, most likely due to acetaldehyde oxidation [38a, 100a, 262]. The formation of acetaldehyde and acetic acid by photocatalytic ethanol dehydrogenationoxidation processes has been recently coupled to condensation reactions in a cascade fashion leading to the selective formation of benzimidazoles of pharmaceutical interest, thus demonstrating that these processes may be applicable to green organic syntheses [338].

Complete photoreforming of ethanol, and thus, maximum production of $\mathrm{H}_{2}$, is a distinct possibility provided the appropriate conditions are applied. This has been proved for $\mathrm{Pt} / \mathrm{TiO}_{2}$ photocatalysts when operating under a flow of $\mathrm{Ar}$ as an inert carrier gas being bubbled through an aqueous ethanol solution under simulated sunlight. ${ }^{33,54}$ Time profiles of $\mathrm{H}_{2}$ and $\mathrm{CO}_{2}$ production, shown in Figure 35, reveal that the former is released at rates peaking after short irradiation times, whereas evolution of the latter is delayed. The authors explained this phenomenon by possible retention of $\mathrm{CO}_{2}$ by either adsorption on the photocatalyst surface or by dissolution in the liquid phase. A further possible interpretation can be deduced by considering the generic photoreforming reaction sequence (Figure 18): If one assumes that dehydrogenation into acetaldehyde is the first step, only $\mathrm{H}_{2}$ would be released into the gaseous phase during the initial stages of the process, whilst subsequent reactions 
entailing the photoreforming of acetaldehyde by photo-generated holes (or hydroxyl radicals) would cause $\mathrm{CO}_{2}$ to evolve at latter stages. As seen in Figure 35, fluctuations in $\mathrm{H}_{2}$ and $\mathrm{CO}_{2}$ production are observed throughout irradiation, a fact presumably caused by the rise and decline of different intermediate species having diverse propensity for participating in charge transfer reactions. Finally, after $\mathrm{H}_{2}$ evolution rates dropped to zero, significant (yet decreasing) $\mathrm{CO}_{2}$ generation was still observed. A possible reason behind this fact might be related to the adsorption of carbonate species on the surfaces of titania, albeit slow oxidation of intermediate carbon species cannot be ruled out. Regarding the photoreforming mechanism, the complete, stoichiometric transformation of ethanol into $\mathrm{H}_{2}$ and $\mathrm{CO}_{2}$ demonstrates the efficiency of the light-induced reforming process on $\mathrm{TiO}_{2}$ materials, including critical steps such as $\mathrm{C}-\mathrm{C}$ cleavage and the subsequent oxidation of the resulting methyl radicals, in competition with coupling reactions leading to alkanes (chiefly methane and ethane).

The initial charge transfer reaction between ethanol and a photo-generated hole results in oxidation of the former into a radical and annihilation of the latter. As described in Section 4.4.2, the ethanolderived radical is likely to occur as the 1-hydroxyethyl species. Coupling of these radicals to yield 2,3-butanediol under UV-vis irradiation has been described [97a, 231a]. In one of these studies, the authors claimed that low surface area titania in $\mathrm{Pt} / \mathrm{TiO}_{2}$ favoured the coupling reaction, which proceeded more effectively than further oxidation, enabling isolation of 2,3-butanediol (selectivities up to $96.6 \%$, see Figure 36). In contrast, high surfaces areas were related to selective conversion into acetaldehyde, acetic acid and $\mathrm{CO}_{2}$, presumably due to enhanced oxidation of retained intermediate radicals within inter-particle porosity. Chen et al. described an analogous process from methanol and ethanol in aqueous media on $\mathrm{ZnS}$ resulting in 1,2-propanediol formation [231a]. These reports illustrate the possibility of preferentially shifting the reaction pathways of alcohols towards radical coupling reactions leading to $\mathrm{C}-\mathrm{C}$ bond formation.

Decarbonylation of acetaldehyde has been suggested as a possible reaction leading to $\mathrm{CH}_{4}$ and $\mathrm{CO}$ $[114,285,342]$. The formation of small amounts of CO has been observed under UV-visible light (Hg lamp) on $\mathrm{Au} / \mathrm{TiO}_{2}$, whereas simulated sunlight resulted in suppressed evolution of such gas, most likely due to the lack of short wavelength frequency photons $(<300 \mathrm{~nm})$, whereas methane, ethane and ethylene were minor co-products under both light irradiation conditions [100a]. Control experiments in the absence of the photocatalyst resulted in limited yet measurable evolution of $\mathrm{H}_{2}$, $\mathrm{CH}_{4}, \mathrm{C}_{2} \mathrm{H}_{4}, \mathrm{C}_{2} \mathrm{H}_{6}, \mathrm{CO}$ and $\mathrm{CO}_{2}$ under UV-visible light, whilst the photoreforming process was predominant in the presence of $\mathrm{Au} / \mathrm{TiO}_{2}$. A thorough study on the photoreforming of ethanol and other biomass-derived oxygenated substrates led to conclude that suppression of $\mathrm{CO}$ production was 
enabled by tin and ruthenium deposits on the titania support by enhancing oxidation processes [283]. It should be noted that irradiations in that work were performed using UV-rich light, a factor which may favour photolytic events. Fluorine-doped $\mathrm{Co}_{3} \mathrm{O}_{4}$ layers prepared by chemical vapour deposition were proven to be highly photoactive for the production of $\mathrm{H}_{2}$ from aqueous ethanol with concomitant and notable production of acetaldehyde, $\mathrm{CO}_{2}$ and methane; proportionally higher $\mathrm{CH}_{4}$ production rates were measured under simulated sunlight relative to UV [197]. Ethylene was produced at significant yields by using $\mathrm{CuO}_{x} / \mathrm{TiO}_{2}$ as the photocatalyst, a fact which was attributed to the presence of acidic sites on titania surfaces [277].

As exemplified in this section, light-activated transformation of ethanol on appropriate photocatalysts may follow different routes at varied degrees of selectivity. Dehydrogenation into acetaldehyde and $\mathrm{H}_{2}$, radical coupling towards $\mathrm{C}-\mathrm{C}$ bond formation, and partial or complete photoreforming have been described in the literature under different irradiation conditions and using different materials. Ultimately, it appears that photocatalytic technologies provide diverse reaction pathways and may enable production of $\mathrm{H}_{2}$ at high selectivities and/or yields.

7.1.3. Glycerol and other polyols. The rapid development of the biofuels industry involving multiton scale production of fatty acid methyl or ethyl esters has brought about a large surplus in the generation of glycerol as a side product with limited market use, and therefore, drastic price decreases [10]. It is predicted that production of glycerol may reach $1.310^{7} \mathrm{t} \mathrm{a}^{-1}$ in 2022 [337]. Other polyols can be readily obtained by the catalytic hydrogenation of saccharides: Sorbitol, mannitol or xylitol are derived from glucose, fructose or xylose, respectively, to name a few examples of such processes [10]. These polyhydroxylated molecules have been studied as versatile feedstocks for the production of fuels [1a-d, 17a] and chemicals [10]. One of the most relevant primary processes among glycerol valorisation schemes is the reforming to generate gaseous mixtures containing $\mathrm{H}_{2}$ [337, 343]. The production of synthesis gas is a compelling approach regarding subsequent transformation to liquid fuels by Fischer-Tropsch technologies, whereas complete aqueous phase reforming of polyols bears the potential of maximising $\mathrm{H}_{2}$ yields and minimising CO production [17a, 17c, 21].

Interest in the photocatalytic reforming of glycerol is experiencing a notable boost owing to the high selectivities and efficiencies attainable by such technology [337, 344]. In contrast to thermocatalytic aqueous phase reforming, which entails side pathways generating methane and other alkanes as by-products, essentially quantitative (stoichiometric, see equation 21) production of $\mathrm{H}_{2}$ has been proven by means of photoreforming [9, 24, 39a, 345]. From an economical and 
environmental perspective, the photocatalytic production of $\mathrm{H}_{2}$ from crude glycerol represents a valuable opportunity, whilst also a technological challenge due to the impurities present [276, 287].

$$
\mathrm{C}_{3} \mathrm{H}_{8} \mathrm{O}_{3}+3 \mathrm{H}_{2} \mathrm{O} \rightarrow 7 \mathrm{H}_{2}+3 \mathrm{CO}_{2} \quad\left(\triangle G^{0}=5.3 \mathrm{~kJ} \mathrm{~mol}^{-1}\right)
$$

On the quest of high $\mathrm{H}_{2}$ production rates by photocatalysis, a large number of researchers have turned their eyes to glycerol as an effective substrate, due to its availability and low price. Data on the photoreforming of glycerol, including the production of $\mathrm{H}_{2}, \mathrm{CO}_{2}$ and several intermediates and by-products are summarised in Table 4. Glycerol possesses three hydroxyl groups prone to dehydrogenation, and thus might enable the attainment of $\mathrm{H}_{2}$ yields surpassing those of other alcohols and polyols, as reported by Bowker and co-workers [90b, 346]. Reports comparing performance under similar conditions reveal that ethanol can produce $\mathrm{H}_{2}$ at faster rates than glycerol (as for example ca. 5.4 and $2.8 \mathrm{mmol} \mathrm{g}_{\mathrm{cat}}{ }^{-1} \mathrm{~h}^{-1}$, respectively, for irradiations of $\mathrm{Pt} / \mathrm{TiO}_{2}$ suspensions in 1.0-1.1 M aqueous solutions) [9]. These apparent inconsistencies may not be such if one considers the variability of different experimental set-ups, and in general, the ranges of maximum $\mathrm{H}_{2}$ production rates are around the same order as those for methanol and ethanol. It is worth reminding here that glycerol concentration may exert a significant influence on $\mathrm{H}_{2}$ production rates (see Section 6.4); several concordant reports point to drastic decreases in activity at increasing concentrations in glycerol-rich regimes [330, 332]. Regarding highly active systems, Shankar and co-workers have reported noticeable efficiencies (around or above $10^{2} \mathrm{mmol} \mathrm{gat}^{-1} \mathrm{~h}^{-1}$ ) for photocatalysts based on titania-deposited copper nanoparticles using fairly concentrated (5\% by volume) glycerol solutions in water, under either Hg lamp light [301] or natural sunlight [336]. Regarding the reduction half-reaction, glycerol was suggested as the primary source of protons for reduction on titania bearing nickel co-catalysts, as inferred by the large proportion of $\mathrm{H}_{2}$ produced from reactions in $\mathrm{D}_{2} \mathrm{O}$ [280].

Glycerol is a widely studied, paradigmatic example of complex oxygenates, and thus, lessons learned from mechanistic investigations on its photoreforming may be of utility for other counterparts. Theoretically, transformation of a substance of this class bearing multiple oxygenated functional groups by strict reforming into $\mathrm{H}_{2}$ and $\mathrm{CO}_{2}$ should proceed as sketched in Figure 18 [272c]. Lalitha et al. suggested a consistent reaction sequence for glycerol (Figure 37) [272c, 337]. Obviously, and as stressed in Section 4.5, a range of side reactions, such as radical coupling, dehydrations or decarbonylation (to generate $\mathrm{C}-\mathrm{H}$ or $\mathrm{C}-\mathrm{C}$ bonds, double $\mathrm{C}=\mathrm{C}$ bonds and $\mathrm{CO}$, respectively) may take place during irradiation. Among the numerous reaction intermediates, some may remain adsorbed on the photocatalyst surface, some others may be too labile and thus 
consumed rapidly via further reforming events, and only the most stable would desorb and become detectable.

Kondarides and co-workers studied in-depth the mechanism of glycerol photoreforming on $\mathrm{Pt} / \mathrm{TiO}_{2}$ by a combination of chromatographic and spectroscopic techniques [345]. The only products detected in the gaseous phase were $\mathrm{H}_{2}$ and $\mathrm{CO}_{2}$, whereas acetaldehyde, ethanol, acetol and methanol (in decreasing production rate order, see Table 4) were found in the liquid phase at early stages (and then consumed), in addition to traces of glyceraldehyde, glycolaldehyde, acetone and acrolein. Furthermore, DRIFTS studies revealed the presence of adsorbed carboxylate species. They rationalised that dehydration and hydrogenation reactions might be responsible for the formation of acetol, in turn giving rise to other species bearing terminal methyl groups. Furthermore, comparison of the outcomes of aerobic and anaerobic irradiations revealed that the oxidation pathways are essentially common to both processes. Further work by the same group using $\mathrm{Cu} / \mathrm{TiO}_{2}$ photocatalysts described fluctuations in $\mathrm{H}_{2}$ and $\mathrm{CO}_{2}$ evolution related to cyclic changes in the oxidation state of copper, although the different photoreforming rates of the structurally varied intermediates might have also had an influence [272b]. Fornasiero and co-workers examined the speciation of liquid phase intermediates of the photoreforming of glycerol on $\mathrm{Cu} / \mathrm{TiO}_{2}$ under $\mathrm{UV}$ visible light; they detected dihydroxyacetone and glycolaldehyde and minor amounts of glyceraldehyde, ethylene glycol, acetol and dimethyl-1,4-dioxane [126, 277]. The multiple detected intermediates and the variability in their formation rates corroborate the complexity of the oxidation reaction sequence for glycerol, and by extension, for any polyhydroxylated substance. Intermediates tend to be formed and consumed rapidly, a fact which often prevents their identification; in particular, carboxylic derivatives occur tightly bound to the photocatalys surfaces, whereas aldehydes and polyols might desorb into the liquid phase to a certain extent.

The formation of $\mathrm{CO}$ is believed to originate in thermal dehydration reactions, as is the case for the conversion of formic acid [337]. Significant amounts of $\mathrm{CO}$ and $\mathrm{CH}_{4}$ were obtained on $\mathrm{NiO}_{x} / \mathrm{TiO}_{2}$, a fact which might be in part due to the strong UV-vis light source (500 W Hg lamp) used [280, 347]. The authors reported the absence of intermediates in the liquid phase, probably owing to their high reactivity, and thus, fast consumption. A less powerful Hg lamp resulted in the absence of evolved $\mathrm{CO}$ and small amounts of $\mathrm{CH}_{4}$ [277]. A highly active $\mathrm{Sn} / \mathrm{RuO}_{2} / \mathrm{TiO}_{2}$ photocatalyst also promoted the evolution of $\mathrm{CO}$ and lower amounts of $\mathrm{CH}_{4}$ under similar irradiation conditions [283].

Ethylene glycol, the simplest polyol, has interest in photoreforming regarding mechanisms and reaction pathways. Despite not being a major biomass product, pyrolysis bio-oils usually contain small amounts of ethylene glycol (Table 1) [19-20]. In addition, it may be produced as an 
intermediate derived from biomass-derived substrates such as ethanol or glycerol. The presence of two hydroxyl groups gives rise to notable activities towards photocatalytic $\mathrm{H}_{2}$ production (see Table 4). For example, UVA irradiation on $\mathrm{Au} / \mathrm{TiO}_{2}$ suspensions has been reported to yield $\mathrm{H}_{2}$ at rates up to $23 \mathrm{mmol} \mathrm{g}_{\mathrm{cat}}^{-1} \mathrm{~h}^{-1}$ ) [115]. Light of lower UV intensity may also induce significant evolution of $\mathrm{H}_{2}$ using $\mathrm{Pt} / \mathrm{TiO}_{2}$ as the photocatalyst [108d]. The formation of other photoreforming products of ethylene glycol has been scarcely studied. Oxidation half reactions would presumably generate glycolaldehyde as the primary dehydration product, and further transformation would follow the general trends described above for glycerol. By using a mechanical mixture of platinum powder and anatase, nearly equimolar amounts of $\mathrm{H}_{2}$ and glycolaldehyde were produced under UV-visible light, in addition to a smaller amount of acetaldehyde which might result from a dehydrationhydrogenation sequence [59].

Higher polyols derived from saccharides by hydrogenation are also interesting candidates for photocatalytic $\mathrm{H}_{2}$ production. Their validity as hydrogen sources by reforming is reinforced by the large number of adjacent hydroxyl moieties. On one side, possibilities for multiple dehydrogenation steps would assumedly lead to fast and selective evolution of $\mathrm{H}_{2}$ into the gas phase at early stages. Moreover, the structural disposition of the hydroxyls enables the oxidation processes involved in photoreforming to proceed smoothly [39a]. As a matter of fact, long term irradiations of polyols, such as erythritol and arabitol, tend to result in the essentially stoichiometric production of $\mathrm{H}_{2}$ and $\mathrm{CO}_{2}$, and thus, maximum yields of the former [39a]. Selected data for these experiments are listed in Table 4.

7.1.4. Comparison to other alcohols and polyols. Photoreforming efficiencies and selectivities of hydroxylated molecules are clearly dependent on their structure. Direct comparisons under analogous conditions have allowed trends and patterns to be outlined. It has been proposed that the reactivity of alcohols varies in the order secondary > primary > tertiary [59]. However, different researchers have concluded differently, stating primary alcohols as the most efficient for $\mathrm{H}_{2}$ evolution $[43,104]$. The number and proximity of hydroxyl groups in a given carbon backbone also determines the distribution of reaction products. In this context, UV-vis light irradiations in the presence of $\mathrm{Pt} / \mathrm{TiO}_{2}$ resulted in a predominantly reforming reaction pathway for glycerol, in contrast to 1,3-propanediol or 2-propanol, which evolved to yield radical coupling products such as methane and ethane [80a, 96]. Along the same lines, a systematic study on a number of hydroxylated substrates using $\mathrm{Pd} / \mathrm{TiO}_{2}$ photocatalysts revealed that alcohols bearing isolated $\mathrm{OH}$ groups, and conversely, higher proportions of aliphatic moieties, produced more alkanes. For example, glycerol yielded mainly $\mathrm{H}_{2}$ and $\mathrm{CO}_{2}$, whereas 1,2-propanediol and 1-propanol also produced $\mathrm{CH}_{4}$ and $\mathrm{C}_{2} \mathrm{H}_{6}$, 
respectively [90]. Shiragami et al. drew equivalent conclusions from their study on a range of different hydroxyl-containing molecules and suggested a step-wise oxidation mechanism consistent with that depicted in Figure 18 [39a]. They also suggested that structures with neighbouring hydroxyl groups, such as biomass-derived polyols and saccharides, are more efficient photoreforming substrates. Independent investigations by several researchers have agreed on the higher activity towards $\mathrm{H}_{2}$ production for polyols as compared to counterparts having less hydroxyl groups [90b, 115, 130]. However, on other occasions low molecular weight primary alcohols (especially methanol) produced $\mathrm{H}_{2}$ at higher rates [70, 108d].

\subsection{Photoreforming of aldehydes}

As compared to alcohols, substrates containing carbonyl groups, that is, aldehydes and ketones, participate in reforming events from a completely different starting point. Hydroxyl groups may rapidly evolve under appropriate photocatalytic conditions to produce $\mathrm{H}_{2}$, as stressed in the previous section. Conversely, carbonyl groups do not provide a direct transformation route to generate the protons required for reduction to $\mathrm{H}_{2}$. Instead, they must undergo a previous electron abstraction step and (at least) either a C-H or C-C bond cleavage in the case of aldehydes or ketones, respectively (see Figure 18). For that reason, their peak $\mathrm{H}_{2}$ production rates are generally lower than those of alcohols and polyols.

Regarding their availability as biomass-derived feedstocks, simple aldehydes and ketones are not primarily encountered - as opposed to more complex aldoses and ketoses which pertain to the class of saccharides - albeit they are contained at significant levels in pyrolysis bio-oils (Table 1) [1920], as many other oxygenates. In fact, they may make up 20-30\% of the total mass of bio-oils by weight; for example, glycolaldehyde, acetaldehyde, hydroxyacetone, glyoxal, formaldehyde or lactaldehyde are contained in high proportions (up to $13,8,7,5,3$ or $2 \%$ ). This latter fact makes them worthy of study for the photocatalytic $\mathrm{H}_{2}$ production regarding a possible valorisation approach for bio-oils. Nonetheless, carbonyl compounds (especially aldehydes) are labile and participate in oxidation, condensation or polymerisation reactions, rendering the bio-oil a fairly unstable medium even upon storage at room temperature [20]. Therefore, photoreforming of at least the water-miscible portion of these biorefinery streams before bulk degradation might be a viable solution regarding energy generation.

7.2.1. Formaldehyde. The simplest (and unique $\mathrm{C}_{1}$ ) aldehyde, i.e. formaldehyde, is a relatively abundant intermediate in biomass processes. Its free form is not a primary biomass derivative. Rather, it readily transforms into other substances by a plethora of reactions. Hydration in water and alcohol addition yield methanediol and hemiacetals/acetals, respectively, without modification of 
the carbon atom oxidation state. Pyrolysis bio-oils may contain formaldehyde in amounts around or above $3 \%$ by weight [19-20]. Furthermore, oxidation and reforming processes of biomass-derived oxygenated substances usually entail the formation of formaldehyde as an intermediate. Photocatalytic examples of these transformations are not exceptions [38b, 66d, 76-77, 116b, 328].

The conversion of formaldehyde by reforming involves the participation of one equivalent of water and the formation of $\mathrm{H}_{2}(2 \mathrm{eq})$ and $\mathrm{CO}_{2}(1 \mathrm{eq})$. The overall transformation can be deconstructed into two elementary steps: (1) partial reforming into $\mathrm{H}_{2}$ and $\mathrm{HCO}_{2} \mathrm{H}$, and (2) dehydrogenationdecarboxylation of $\mathrm{HCO}_{2} \mathrm{H}$ into $\mathrm{H}_{2}$ and $\mathrm{CO}_{2}$. Methanol reforming theoretically proceeds via a formaldehyde pathway (see Figure 33); condensation of both species may lead to the formation of hemiacetal and acetal adducts. Analogously, formaldehyde generation is possible for any higher oxygenate following the reaction cascade shown in Figure 18. Thus, the study of formaldehyde photoreforming has interest regarding fundamental mechanistic investigations, as well as per se if the photocatalytic production of $\mathrm{H}_{2}$ from pyrolysis oils is considered. The relatively scant literature on this topic is reviewed herein, and the most relevant data are listed in Table 5.

Shiragami et al. demonstrated that the stoichiometric production of $\mathrm{H}_{2}$ by formaldehyde photoreforming is achievable by using $\mathrm{Pt} / \mathrm{TiO}_{2}$ photocatalysts and a $\mathrm{UV}$-vis light source [39b]. The efficiency in $\mathrm{H}_{2}$ production is apparently lower by one or two orders of magnitude (values on the order of $1 \mathrm{mmol} \mathrm{g}_{\mathrm{cat}}{ }^{-1} \mathrm{~h}^{-1}$, see Table 5) than in the case of alcohols. However, outstanding performances have been reported for some systems based on simple oxides other than titania and in the absence of co-catalysts. For example, bare $\mathrm{Cu}_{2} \mathrm{O}$ microcrystals have served as efficient photocatalytic materials for the production of $\mathrm{H}_{2}\left(2.7 \mathrm{mmol} \mathrm{g}_{\mathrm{cat}}{ }^{-1} \mathrm{~h}^{-1}\right)$ from aqueous formaldehyde under UVA light [348]. Intermediate formate species were also detected in the liquid phase. Another simple oxide, $\mathrm{ZnO}$, promoted the evolution of $\mathrm{H}_{2}$ from aqueous formaldehyde (or methanol) at high rates (> $30 \mathrm{mmol} \mathrm{g}_{\mathrm{cat}}^{-1} \mathrm{~h}^{-1}$ ). These two examples indicate that formaldehyde (and by extension, probably higher aldehydes too) might be sensible to the nature of the oxide semiconductor, probably entailing different photoreforming (especially regarding oxidation processes) routes. Moreover, Matsumura et al. studied the photoreforming of methanol, formaldehyde and formic acid on $\mathrm{Pt} / \mathrm{CdS}$ materials. Interestingly, they only observed the formation of $\mathrm{H}_{2}$ and traces of methanol-claimed to be the result of disproportionation events-from formaldehyde, whereas formic acid also yielded $\mathrm{CO}_{2}$ and $\mathrm{CO}$ [218].

Mechanistic studies on formaldehyde photoreforming are scarce, and it would be thus worth to extend them aiming at a deeper understanding of the underlying phenomena behind the fate of more complex biomass-derived oxygenates at the latter steps of their photocatalytic transformation. 
7.2.2. Acetaldehyde. Although it is not strictly speaking a primary biomass derivative, acetaldehyde can be readily generated both in nature as a metabolite and artificially from renewable resources. Furthermore, pyrolysis bio-oils may contain significant amounts (approaching 10\% by weight) of its free or adduct variants [19-20]. From this perspective, research on the photocatalytic production of $\mathrm{H}_{2}$ from acetaldehyde is a stimulating — yet seldom explored—task.

The first step in ethanol photoreforming is generally dehydrogenation, whereby oxidation to acetaldehyde takes place. Its detection and quantification under such circumstances has been reported by a number of independent research teams and under varied experimental conditions [43, 59, 70, 100a, 100d, 120c, 197, 262, 268b, 277, 288, 349]. Therefore, the series of subsequent reactions are coincident (see Section 7.1.2) and starts with oxidation to acetic acid, which in turn tends to evolve by decarboxylation. The presence of the methyl group generates a diversity of possible transformations under irradiation on typical photocatalysts. Methyl radicals produced by decarboxylation would remain adsorbed to the surface of the solid and may undergo either further oxidation or coupling to other radicals. If the latter species is the hydrogen radical, methane would be formed. On the other hand, coupling to a second methyl would result in the generation of ethane. This will be discussed in more detail in the Section devoted to acetic acid (7.3.2) below. In the theoretical scenario of complete reforming, three equivalents of water would be consumed and five and two equivalents of $\mathrm{H}_{2}$ and $\mathrm{CO}_{2}$, respectively, would be produced (see equation 22).

$\mathrm{CH}_{3} \mathrm{CHO}(g)+3 \mathrm{H}_{2} \mathrm{O}(l) \rightarrow 5 \mathrm{H}_{2}(g)+2 \mathrm{CO}_{2}(g) \quad \triangle G^{0}=-55.9 \mathrm{~kJ} \mathrm{~mol}^{-1}$

Data for acetaldehyde photoreforming are shown in Table 5, where the fact that only a few reports are available is apparent. Patsoura et al. specifically studied the fate of acetaldehyde in aqueous $\mathrm{Pt} / \mathrm{TiO}_{2}$ suspensions under simulated solar light, and concluded that $\mathrm{H}_{2}$ production rates are substantially lower than for alcohols (including methanol and ethanol) and acids (formic and acetic) [38a]. As shown in Figure 38, the rate of production fluctuates over long term irradiation: Initially it increases and rapidly reaches a plateau to plummet later; nonetheless, a second stage of $\mathrm{H}_{2}$ evolution is observed at reaction times between 6 and $20 \mathrm{~h}$. This may indicate that different intermediates are likely to undergo photoreforming at different rates. However, the total amount of $\mathrm{H}_{2}$ produced was only a fraction $(\mathrm{ca} .1 / 4)$ of the total predicted by the stoichiometry of overall reforming, as opposed to acetic acid, which is essentially converted into the maximum amount of $\mathrm{H}_{2}$ [38a]. Mao and co-workers also compared the photoreforming efficiency of ethanol, acetaldehyde and acetic acid under UV-rich light [120a]. When using ethanol as the substrate, $\mathrm{H}_{2}$ production decelerated with time, a phenomenon which was ascribed to the lower activity of the acetaldehyde and acetic acid intermediates formed. In contrast to the aforementioned study, they observed higher 
rates for acetaldehyde than for the acid. This trend is in agreement with another report dealing with $\mathrm{Pd} / \mathrm{TiO}_{2}$ as the photocatalytic material [90b].

It appears that acetaldehyde and formaldehyde - and probably other aldehydes too-perform less efficiently than alcohols for photocatalytic $\mathrm{H}_{2}$ production. In addition to the absence of hydroxyl groups, a plausible reason behind this observation is the tight adsorption of distinct species (i.e. dioxymethylene moieties formed by condensation of the initial aldehyde on oxide supports, or carboxylate species derived from oxidation) on the photocatalyst surface. Further spectroscopic and mechanistic studies might provide answers to these uncertainties.

7.2.3. Other aldehydes and ketones. Carbonyl groups are ubiquitous in biomass components, although mostly in the form of cyclic aldoses and ketoses in saccharides. Photoreforming of those compounds will be discussed in the following section. Free aldehydes and ketones may be present in pyrolised lignocelluloses, and as such, some interest may lie in their role in the potential photocatalytic production of $\mathrm{H}_{2}$ from bio-oils. Glycolaldehyde is a particularly abundant chemical in pyrolysis oils (see Table 1) and may thus be considered as a model substrate for photoreforming [19-20].

Production of $\mathrm{H}_{2}$ on $\mathrm{Pt} / \mathrm{TiO}_{2}$ photocatalysts under UV-visible light from aqueous glyceraldehyde, which is likely to be a primary intermediate in the reforming of glycerol, has been studied [39b]. Essentially complete reforming was observed, a fact which reflects the tendency of the substrate to efficiently undergo transformation by photocatalysis yielding the theoretical maximum amount of $\mathrm{H}_{2}$. However, production rates were considerably lower (ca. $2.2 \mathrm{mmol} \mathrm{gat}^{-1} \mathrm{~h}^{-1}$, Table 5) than those of the parent glycerol (ca. $5.1 \mathrm{mmol} \mathrm{g}_{\mathrm{cat}}{ }^{-1} \mathrm{~h}^{-1}$, Table 4). Pichat et al. observed that propanal reacted at about two orders of magnitude slower rates that its parent alcohol (1-propanol) on $\mathrm{Pt} / \mathrm{TiO}_{2}$, thus confirming the more limited propensity for photoreforming of aldehydes as compared to alcohols [43].

\subsection{Photoreforming of carboxylic acids}

The carboxylic functionality is, in addition to hydroxyl and carbonyl, one of the oxygen-containing motifs featuring distinct adsorption and reactivity phenomena during photoreforming. Carboxylates tend to bind strongly to classical oxide semiconductor (e.g. $\mathrm{TiO}_{2}$ ) surfaces, provided the electrostatic attraction is favourable. In this line, high $\mathrm{pH}$ values disrupt such interaction and provoke detrimental effects on efficiency (see Section 6.3). The initial reaction in the photoreforming sequence is decarboxylation, and as a result, $\mathrm{CO}_{2}$ release is expected even at short irradiation times, presumably accompanied by proton reduction. 
Components of raw biomass, chiefly hemicellulose, contain simple carboxylic compounds forming part of complex biomacromolecules as esters. It is not surprising then that processing (e.g. hydrolysis, pyrolysis) of lignocelluloses leads to the release of carboxylic acids, being acetic acid the most abundant example, followed by formic. Fermentation of saccharides also renders broths rich in carboxylic compounds, among which lactic acid is the most prominent example [10]. Furthermore, carboxyl groups are expected to form, at least transiently, by the photoreforming of any oxygenated substrate. In this section, a summary of this area of research is given (see data in Table 6).

7.3.1. Formic acid. According to the photoreforming sequence depicted in Figure 18, formic acid is the latter intermediate before complete mineralisation of oxygenated organic backbones regardless of which substrate is taken into account. Therefore, it is a model compound in oxidation or reforming processes. In the latter case, the unique pathway it can take is its decomposition (decarboxylation/dehydrogenation) into $\mathrm{H}_{2}$ and $\mathrm{CO}_{2}$ (equation 23).

$$
\begin{array}{ll}
\mathrm{HCO}_{2} \mathrm{H}(l) \rightarrow \mathrm{H}_{2}(g)+\mathrm{CO}_{2}(g) & \triangle G^{0}=-33.0 \mathrm{~kJ} \mathrm{~mol}^{-1} \\
\mathrm{HCO}_{2} \mathrm{H}(l) \rightarrow \mathrm{H}_{2} \mathrm{O}(l)+\mathrm{CO}(g) & \triangle G^{0}=-12.9 \mathrm{~kJ} \mathrm{~mol}^{-1}
\end{array}
$$

Photocatalysts ranging from $\mathrm{TiO}_{2}$ or other oxides to $\mathrm{CdS}$ can enable the decomposition of formic acid into $\mathrm{H}_{2}$ and $\mathrm{CO}_{2}$ at comparable rates. The classical $\mathrm{Pt} / \mathrm{TiO}_{2}$ composite readily catalyses the reaction under UV-visible light either in gaseous [315, 328] or liquid phases [218], whereas photocatalysts based on copper oxides [193] or cadmium sulfide [224] also perform suitably under visible light. The action of simulated solar light on aqueous $\mathrm{Pt} / \mathrm{TiO}_{2}$ suspensions results in very sharp rise in $\mathrm{H}_{2}$ production and much more rapid decomposition than for acetic acid (see Figure 38) [38a]. Formic acid decomposition on $\mathrm{Pd} / \mathrm{TiO}_{2}$, even in the absence of light, has been proven [90b]. The highest $\mathrm{H}_{2}$ production rate ( $c$ a. $20 \mathrm{mmol} \mathrm{g}_{\mathrm{cat}}{ }^{-1} \mathrm{~h}^{-1}$, Table 6) was reported by using a mixture of platinum black and CdS; inclusion of selenium in the form of solid solution proved detrimental for the reaction [235].

One of the possible side reactions involving formic acid is dehydration to generate $\mathrm{CO}$ and $\mathrm{H}_{2} \mathrm{O}$ (equation 24). Kakuta and Abe claimed that whereas $\mathrm{Pt} / \mathrm{Cu}_{2} \mathrm{O}$ or bare $\mathrm{Cu}_{2} \mathrm{O}$ effectively induced photoreforming, CdS led to the formation of significant amounts of CO under visible light [193]. Independent investigations confirmed this phenomenon for a photocatalytic system based on a mechanical mixture of platinum and cadmium sulfide [218]. Lower proportions of CO were formed using $\mathrm{Pt} / \mathrm{TiO}_{2}$ despite the fact that the irradiation used was rich in UV frequencies [218]. 
Formic acid is a peculiar oxygenated substrate regarding photocatalytic $\mathrm{H}_{2}$ production. In general, it readily reacts by releasing $\mathrm{H}_{2}$ and $\mathrm{CO}_{2}$, yet in the presence of chalcogenide materials or under UVrich light $\mathrm{CO}$ evolution has been observed. As a substrate, its importance is confined to the use of bio-oils as feedstocks. From a fundamental perspective, the role played by formic acid might be interesting regarding mechanisms or adsorption and deactivation events.

7.3.2. Acetic acid. The use of acetic acid for the photocatalytic production of $\mathrm{H}_{2}$ entails selectivity issues, given its tendency to decompose by the photo-Kolbe process into $\mathrm{CO}_{2}$ and $\mathrm{CH}_{4}$, instead of reacting via preferable reforming pathways. Similar difficulties have been encountered in the steam reforming approach (where it has been used as a model compound for bio-oils), which in addition entails the generation of $\mathrm{CO}$ as a by-product [18]. Acetic acid is commonly produced to some extent in most processed raw biomass streams, including pyrolysis oils and hydrolysates of lignocellulosic feedstocks.

In the early years of the development of photocatalysis, Bard and co-workers observed that aliphatic carboxylic (including acetic) acids underwent fast decarboxylation on illuminated aqueous $\mathrm{Pt} / \mathrm{TiO}{ }_{2}$ suspensions [89a, 107], causing the preferential production of $\mathrm{CO}_{2}$ and $\mathrm{CH}_{4}$. In this photo-Kolbe scenario, the formation of methyl radicals was proven by EPR spectroscopy [68a, 89b]. Further coupling with hydrogen radicals yields methane, whereas homo-coupling yields ethane (see Figure 17). This dichotomy between both mechanisms was also raised by Falconer and co-workers, based on the study of gas phase reactions on bare titania [350]. Particularly high selectivities towards ethane were reported by using a photoelectrochemical set-up, where methyl and hydrogen radicals are expected to be generated on different electrodes [351]. Sato and co-workers reported similar results regarding high ethane selectivities by gas phase reactions on $\mathrm{UV}$-irradiated $\mathrm{Pt} / \mathrm{TiO}{ }_{2}$ in cases where water vapour was deliberately added, presumably due to the favoured hydride abstraction by $[\mathrm{OH}]^{\bullet}$ species [108c, 315]. In none of these and similar reports did the authors consider photoreforming of acetic acid, albeit they noticed imbalances if only photo-Kolbe events were considered and attributed them to the oxidation of minor amounts of ethanol and acetaldehyde, presumably formed from the substrate [44a, 89b].

Abrahams et al. observed intermediate situations between photo-Kolbe and regimes where $\mathrm{H}_{2}$ production was enhanced, also using $\mathrm{Pt} / \mathrm{TiO}_{2}$ as photocatalyst. They claimed that materials containing mixed anatase and rutile (at high proportions of the former) were more active and more selective towards $\mathrm{H}_{2}$ [119]. A study on the photocatalytic oxidation of acetic acid revealed that abstraction of hydrides on the $\alpha$ carbon by photo-generated $[\mathrm{OH}]^{\cdot}$-instead of direct decarboxylation-took place on $\mathrm{TiO}_{2}$, as suggested by EPR spectroscopy data [352]. An 
unambiguous proof to this hypothesis was delivered by Sakata et al. in his thorough examination of the photocatalytic reactions of carboxylic acids and water on several photocatalysts under UVvisible (> $320 \mathrm{~nm}$ ) irradiation [37]. They demonstrated that pathways other than the photo-Kolbe route took place and were favoured at high $\mathrm{pH}$. In fact, formation of alkanes $\left(\mathrm{CH}_{4}\right.$ and $\left.\mathrm{C}_{2} \mathrm{H}_{6}\right)$ was practically suppressed in basic solutions, probably due to more favourable oxidation of water or hydroxide anions into hydroxyl radicals, which in turn would oxidise methyl radicals into methanol, and thus reduce their population and availability for radical coupling reactions (Figure 17). This was confirmed by the detection of traces of methanol [37], a key intermediate in the photoreforming series of reactions, as depicted by dashed lines in Figure 18. Other reports describing the formation of methanol or ethanol (among other products) include experiments under UVA irradiation on suspensions of titania-based photocatalysts [168, 333, 353]. Consistent with these findings, complete photoreforming of acetic acid has been reported in systems whereby continuous purging with an inert gas was applied [38a].

In brief, the fate of acetic acid in the presence of irradiated titania photocatalysts appears to be highly dependent on the experimental conditions. The tendency to generate methyl radicals unleashes a range of different possible reaction pathways. Coupling reactions involving methyl radicals yield alkanes, whereas oxidation by $[\mathrm{OH}]^{\bullet}$ species generates methanol, an intermediate which can undergo further reforming. The latter case is greatly promoted in basic aqueous media or under inert gas flow. Thus, photoreforming under appropriate conditions can be an excellent technology towards the efficient production of $\mathrm{H}_{2}$ from acetic acid (and other carboxylic counterparts).

7.3.3. Lactic acid. The production of lactic (2-hydroxypropanoic) acid from biomass is growing at a fast pace in current years [10]. The main process involves the fermentation of saccharides, especially glucose, although chemical routes starting from cellulose are being recently developed [354]. The importance of lactic acid as a platform for the production of a number of chemicals in food, cosmetics and pharmaceutical industries is indisputable. In energy schemes, lactic acid may become a viable source of fuels and fuel additives [1c].

Regarding its use in the photocatalytic production of $\mathrm{H}_{2}$, many researchers have chosen lactic acid as an electron donor for the development of novel active materials (especially in the case of sulfide co-catalysts, see Section 5.5.2). A summary of relevant data in this field is shown in Table 6. From a fundamental perspective, the presence of both hydroxyl and carboxyl residues makes comparative studies on their behaviour under photoreforming conditions pertinent. Two different elementary steps can happen: (i) dehydrogenation of the hydroxyl group to generate pyruvic acid, and (ii) 
decarboxylation leading to $\mathrm{C}_{2}$ derivatives. A representation of these pathways is schematically depicted in Figure 39. In either case, the corresponding oxidation half-reactions are expected to be accompanied by reduction of released protons, and hence, the rates of $\mathrm{H}_{2}$ production will be affected by the efficiency of any of the two routes.

A thorough investigation on the photocatalytic reactions of $\alpha$-hydroxyacids using $\mathrm{TiO}_{2}$ and $\mathrm{CdS}$ as the light-absorbing semiconductors after platinum photodeposition was performed by Harada et al. [84, 215-216]. They observed that the former material mainly favoured decarboxylation, leading to acetaldehyde, acetic acid and ethanol as the derived oxidation co-products (formed at 900, 126 and $39 \mu \mathrm{mol} \mathrm{g} \mathrm{gat}^{-1} \mathrm{~h}^{-1}$, respectively, in addition to $\mathrm{CO}_{2}$ at $1008 \mu \mathrm{mol} \mathrm{g}_{\mathrm{cat}}{ }^{-1} \mathrm{~h}^{-1}$ ), whereas formation of pyruvic acid by the competing route was minor $\left(17 \mu \mathrm{mol} \mathrm{g}_{\mathrm{cat}}{ }^{-1} \mathrm{~h}^{-1}\right)$. In contrast, cadmium sulfide exclusively promoted the dehydrogenation step, as suggested by the formation of pyruvic acid as the only liquid phase product, accompanied by minor formation of $\mathrm{CO}_{2}$. Apparent quantum yields for $\mathrm{H}_{2}$ production reached 71 and $38 \%$ for $\mathrm{Pt} / \mathrm{TiO}_{2}(360 \mathrm{~nm})$ and $\mathrm{CdS}(440 \mathrm{~nm})$, respectively [215]. Several hypotheses based on differing band energy positions or adsorption modes were proposed for this clear selectivity switch [84]. Independent surface studies have revealed that the pyruvic acid product exhibits little tendency to adsorb on $\mathrm{CdS}$, and thus, it would remain stable under irradiated suspensions of the sulfide solid [39b]. Other systems based on cadmium sulfide agree on the exclusive formation of pyruvic acid [99, 355]. All these conclusions are extremely valuable for discerning different photocatalytic behaviours of either oxide or non-oxide (chiefly sulfide) semiconductors. Should the aim be complete reforming, the former (especially $\mathrm{TiO}_{2}$ ) appear as more suitable.

As mentioned above, lactic acid has been selected as a model substrate for the development of highly active photocatalysts for $\mathrm{H}_{2}$ production (see data in Table 6). Despite the presumably lower efficiency of CdS materials for complete photoreforming, they have been proven as more efficient than $\mathrm{TiO}_{2}$ analogues under visible light irradiation. This was the case for a systematic study on alloy nanoparticles as co-catalysts, which displayed significant $\mathrm{H}_{2}$ evolution rates (up to $15.9 \mathrm{mmol} \mathrm{g}_{\text {cat }}{ }^{-1}$

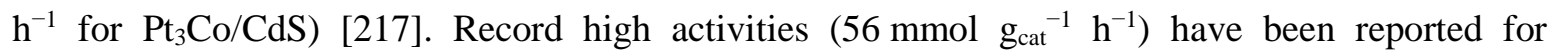
$\mathrm{Pt} / \mathrm{CdS} / \mathrm{RGO}$ where graphene acted as an electron mediator component [221]. Apparent quantum efficiencies are generally elevated for CdS when using lactic acid [84, 99, 215, 294b, 294c], a fact which has unsurprisingly motivated intense research in the design of sulfide photocatalysts [212], mostly promoted after pioneering research by Li and co-workers [291b]. A further point to note is the recent interest raised in other non-oxide materials, mainly metal phosphides, as co-catalysts for $\mathrm{H}_{2}$ production from aqueous lactic acid, enabling rates as high as $200 \mathrm{mmol} \mathrm{g}_{\mathrm{cat}}^{-1} \mathrm{~h}^{-1}$ [297-298]. 
Yet not purely a primary biomass product, recently boosted motivation in studying the photocatalytic production of $\mathrm{H}_{2}$ from lactic acid has resulted in a fruitful area of research concerning materials design. Since the presence of both carboxylic and hydroxyl functionalities is expected during the photoreforming of any oxygenated substrate, a simple molecule containing them-i.e. lactic acid - appears as a perfect model. Indeed, delving into the mechanistic aspects has revealed the distinct performances of the two main semiconductors in photocatalysis, that is, $\mathrm{TiO}_{2}$ and CdS. Whilst the latter shows more limited activity for photoreforming, high $\mathrm{H}_{2}$ production rates can be achieved by rational nanocomposite design.

7.3.4. Other acids. A wide range of compounds containing acidic functionalities might be produced in biorefineries. The above examples (formic, acetic and lactic) have provided valuable information on the mechanisms and efficiencies of photoreforming processes involving carboxylic acid substrates. Comparison to other acids has been undertaken in several investigations to reinforce the derived knowledge.

Discrimination between the photo-Kolbe and the photoreforming pathways (see 7.3.2), based on data for acetic acid, was aided by analogue experiments performed on higher aliphatic carboxylic acids. ${ }^{53}$ Despite the predominant formation of the corresponding alkanes (ethane, propane and butane for propanoic, butanoic and pentanoic acids, respectively, see Table 6) by decarboxylation, $\mathrm{H}_{2}$ formation was observed for the whole series and increased with increasing chain length, thus suggesting that other mechanisms might be at play. Even in the case of fatty acids, $\mathrm{H}_{2}$ evolution activity has been achieved by using the stearic (octadecanoic) counterpart as an example [108a].

Processing of biomass feedstocks may result in the oxidation of saccharides into acidic derivatives. Furthermore, these are also likely to be produced during photocatalytic reactions of the parent substances, and hence, study of their behaviour under such conditions is relevant. Gluconic and threonate salts have been used as substrates for photoreforming on $\mathrm{Pt} / \mathrm{TiO}_{2}$, and both resulted in production of $\mathrm{H}_{2}$ and $\mathrm{CO}_{2}$ [39b].

Oxalic acid is the most highly oxidised form of carbon, with the exception of $\mathrm{CO}_{2}$. Its decomposition should in principle lead to the formation of $\mathrm{H}_{2}$ and $\mathrm{CO}_{2}$ in a 1:2 molar ratio. Photocatalysis is as a viable technology for this process. Oxalic acid produced $\mathrm{H}_{2}$ at faster rates than formic, formaldehyde [81], or ethanol [356] on $\mathrm{Pt} / \mathrm{TiO}_{2}$ under UV-rich light. A dedicated investigation on the system revealed that the fate of oxalic acid is governed by the general features of photoreforming: It is negligible in the absence of metal co-catalyst and kinetics follow a Langmuir-type behaviour [60]. 
Finally, it is worth noting that ascorbic acid is gaining increasing attention as a sacrificial electron donor in photocatalytic $\mathrm{H}_{2}$ producing systems, most likely due to its propensity towards oxidation. Many researchers have used it for the evaluation of homogeneous [236-239, 257b, 304, 306-308], or novel heterogeneous photocatalysts [222, 248, 254-255, 309, 319]. It is accepted that ascorbate is more strongly reducing than the parent acidic form [322]. Despite its utility as an electron donor and its renewable sources, ascorbic acid is a low-production high-cost substrate, and thus, its suitability for mass production of $\mathrm{H}_{2}$ (or other fuels) is questionable.

\subsection{Photoreforming of saccharides}

Since they make up the major proportion of plant biomass as the building block monomers of structural biomacromolecules, saccharides are the focus of most primary transformations in biorefineries [10]. Production of $\mathrm{H}_{2}$ from these crucial platform molecules has been intensely investigated, especially by steam or aqueous phase reforming [1d, 14, 15b, 17c, 21]. In this section, the alternative photoreforming process is reviewed.

Relevant data on the photocatalytic production of $\mathrm{H}_{2}$ using saccharides as the substrates are summarised in Table 7. Given their structural complexity, detailed mechanistic studies regarding their photoreforming are cumbersome, and in general reported data is limited to the evaluation of the performance by quantification of $\mathrm{H}_{2}$. Some researchers have explored the possibility of complete transformation by including $\mathrm{CO}_{2}$ yields in their accounts. Data in Table 7 reveal that production rates tend to be somewhat lower than those obtained for simple oxygenates (i.e. alcohols, aldehydes and acids), most likely due to the presumably more hindered interaction of complex saccharides with photocatalyst surfaces. Nevertheless, relatively high $\left(10^{1-}\right.$ $\left.10^{2} \mathrm{mmol} \mathrm{g}_{\mathrm{cat}}{ }^{-1} \mathrm{~h}^{-1}\right) \mathrm{H}_{2}$ production rates are attainable.

7.4.1. Monosaccharides. From a practical perspective, free monomeric sugars are valuable model substrates for the examination of photoreforming. The multiple hydroxyl groups in their structures guarantee the possibility of $\mathrm{H}_{2}$ generation by dehydrogenation. Complete transformation is feasible [9] via the sequence of steps described in Figure 18. Particular cases for glucose (the most relevant example), other hexoses, and pentoses are outlined below and in Table 7.

Kawai and Sakata first demonstrated the photocatalytic production of $\mathrm{H}_{2}$ from glucose (among other saccharides) in aqueous media [108a]. More recently, the complete photoreforming of glucose and fructose has been proven by using $\mathrm{Pt} / \mathrm{TiO}_{2}$ under simulated solar light and continuous flow of an inert gas [9, 317]. St. John and co-workers had anticipated this in a seminal study on glucose [357]. Significant production of $\mathrm{H}_{2}$ and $\mathrm{CO}_{2}$ was achieved, albeit the quantities were somewhat below the 
theoretical maxima. Nonetheless, extrapolation to the limiting case of infinite dilution revealed that stoichiometric reforming is still possible [39b].

In their pioneering work, St. John et al. noted the potential for light-induced generation of $\mathrm{H}_{2}$ from waste streams generated by saccharide transformations, taking glucose as a model substrate. They stated that a range of hydroxycarboxylic acids derived from partial oxidation or other biomassprocessing treatments (e.g. paper, pulp or sugar) industries are noxious by-products. Moreover, they also claimed these species as "virtually impossible to separate from the aqueous phase, and have such low heat content that they are poor boiler fuels. Their photoelectrochemical (or photocatalytic) gasification to $\mathrm{H}_{2}$ and $\mathrm{CO}_{2}$ using photocatalysts has potential for cleaning up waste streams, while simultaneously providing a source of $\mathrm{H}_{2}$ without the generation of an explosive $\mathrm{H}_{2} / \mathrm{O}_{2}$ mixture" [357]. This reflects the potential of photocatalysis for simultaneous wastewater decontamination and $\mathrm{H}_{2}$ production.

In addition to glucose, other hexoses such as fructose, galactose or mannose have also served as substrates for the photocatalytic production of $\mathrm{H}_{2}$ from aqueous $\mathrm{Pt} / \mathrm{TiO}_{2}$ suspensions [9, 159a, 287, 317]. Activities were similar to those reported for glucose (see Table 7). Pentoses are another class of interesting biomass-derived substances - especially xylose, a hemicellulose monomer-which has received some attention. In particular, xylose [39b, 39c], arabinose [159a, 317] and ribose [317] have been employed; results are similar than those for the rest of studied monosaccharides (see Table 7).

7.4.2. Disaccharides. Disaccharides are valuable substances in food and pharmaceutical industries. A number of examples, such as sucrose or lactose, can be directly produced from crops, whereas others (e.g. cellobiose or maltose) can be produced by hydrolysis of polysaccharides.

Photocatalytic production of $\mathrm{H}_{2}$ from model disaccharides is slightly less efficient than from monomers (see data in Table 7), likely due to their high molecular weight. This unsurprising trend reveals that increasing structural complexity and size is a factor determining the rates of $\mathrm{H}_{2}$ release. Lactose (a glucose-galactose dimer) can be completely transformed by photoreforming on $\mathrm{Pt} / \mathrm{TiO}_{2}$ yielding maximum amounts of $\mathrm{H}_{2}[9,317]$. Production of $\mathrm{H}_{2}$ and $\mathrm{CO}_{2}$ as the only products when using sucrose (a glucose-fructose dimer) as the feedstock under similar conditions suggested that total mineralisation was taking place [36, 358]. Rates of hydrogen evolution as high as $3.1 \mathrm{mmol} \mathrm{g}_{\mathrm{cat}}^{-1} \mathrm{~h}^{-1}$ have been reported from aqueous sucrose under Xe lamp light using nitrogenand boron-doped, platinum-deposited titania materials [359]. Similar yields were achieved on nondoped analogues under UV-rich irradiation [62a]. Photocatalysts based on CdS have also served as relatively efficient materials for the photoreforming of sucrose [214]. By using maltose or 
cellobiose, two glucose dimers, $\mathrm{H}_{2}$ production rates around or above $3 \mathrm{mmol} \mathrm{g} \mathrm{cat}^{-1} \mathrm{~h}^{-1}$ have been reported [9, 317].

7.4.3. Polysaccharides. The principal proportion of plant biomass is composed of polysaccharides - cellulose and hemicellulose. Their use in raw form for the generation of energy and heat has been practiced since millennia. Isolation of cellulose has undoubted industrial and technological importance due to the widespread use of paper. Starch - another glucose polymer - is widely available, albeit its use as feedstock for fuel production is ethically reprehensible given its importance in nutrition. Deconstruction of raw lignocelluloses into monomeric or oligomeric derivatives by hydrolysis is currently opening up a promising strategy towards liquid biofuels. Transformation into $\mathrm{H}_{2}$ can be effected by thermochemical methods under harsh conditions. In this section, the chances of direct photoreforming of polysaccharides for the production of $\mathrm{H}_{2}$ are discussed. Related data are compiled in Table 7.

Both starch and cellulose react to some extent in suspensions containing $\mathrm{Pt} / \mathrm{TiO}_{2}$ photocatalysts irradiated with UV-vis or simulated solar light. Under identical conditions (Xe lamp as the light source), starch produced more hydrogen than cellulose (80 and $13 \mu \mathrm{mol} \mathrm{g} \mathrm{cat}^{-1} \mathrm{~h}^{-1}$, respectively), probably owing to the more intricate supramolecular structure of the latter [108a]. In their pioneering research, Kawai and Sakata demonstrated that, although at low quantum efficiencies, nearly stoichiometric and complete transformation (photoreforming) of both starch and cellulose can be made possible at long irradiation times from aqueous suspensions containing $\mathrm{RuO} / 2 / \mathrm{Pt} / \mathrm{TiO}_{2}$ [36]. They also showed that addition of $\mathrm{NaOH}$ accelerated the evolution of $\mathrm{H}_{2}$. In the particular case of starch, enhanced production rates $\left(4 \mathrm{mmol} \mathrm{g}_{\mathrm{cat}}^{-1} \mathrm{~h}^{-1}\right.$, more than a five-fold increase, UV-vis irradiation) were reported after microwave pretreatment of the substrate [62a]. Even without pretreatment and under simulated sunlight, the efficiency of similar systems was significant [9]. Partial cellulose hydrolysis under acidic conditions is also a viable method for boosting the efficiency of photocatalytic $\mathrm{H}_{2}$ generation (production rates increased from 0.6 to $0.9 \mu \mathrm{mol} \mathrm{gcat}^{-1} \mathrm{~h}^{-1}$ ) [317]. Speltini et al. studied the reaction of cellulose on $\mathrm{Pt} / \mathrm{TiO}_{2}$ under UVA light or simulated solar light [313b]. It is interesting to note that based on analyses of the liquid phases, they identified small amounts of glucose and 5-(hydroxymethylfurfural); they rationalised their formation and subsequent dehydration by hydrolysis on surface acidic sites on titania, respectively. Formation of coloured by-products was believed to be caused by further degradation. The authors claimed that these species might have had a sensitising effect enhancing the photocatalytic process [125]. A specially designed experimental set-up based on a cylindrical corrugated photo-reactor coated with anatase and using concentrated aqueous $\mathrm{ZnCl}_{2}$ media was 
reported to enable the direct transformation of cellulose to 5-(hydroxymethylfurfural) under UV light [360]. The solubilising power of the highly ionic solution facilitated the depolymerisation of cellulose, whereas the high exposed photocatalyst area ensured light harvesting.

Surprisingly, structurally recalcitrant, nearly raw, biomass-derived feedstocks such as cellulose can be directly converted to $\mathrm{H}_{2}$ and other co-products by simple photocatalytic processes and using solar-like irradiation. Photoreforming has been demonstrated on the archetypal benchmark $\mathrm{Pt} / \mathrm{TiO}_{2}$ material. Gentle processing of the substrate enhances production rates. This field is, despite the currently moderate efficiencies, technologically interesting and may be applied to more complex feedstocks or waste materials, as discussed below.

\subsection{Raw biomass feedstocks for photocatalytic $\mathbf{H}_{2}$ production}

Considering economic factors, ideal biomass-to-hydrogen strategies should involve the use of raw or gently processed feedstocks [4a]. Preferred conversion technologies are those whereby low-cost or waste materials are the source of $\mathrm{H}_{2}$. The most suitable processes known are performed under harsh temperature-pressure conditions [4c, 14-15] or require the manipulation of microorganisms (fermentation) [4c, 15b, 361]. Light-activated processes are of relatively lower efficiency than thermal methods, although they entail lower environmental impact [361]. Biomass photoreforming has been proven to perform in a relatively acceptable fashion for complex primary biomass products (see the preceding section). The use of raw feedstocks has been rather unexplored to date, as illustrated in the data listed in Table 8. The account given herein aims at attracting focus towards this technology.

Based on the discovery of cellulose photoreforming on $\mathrm{Pt} / \mathrm{TiO}_{2}$ [36], Kawai and Sakata applied this approach to the generation of $\mathrm{H}_{2}$ from a range of raw biomass products, including algae, rice plant, turf, olive oil, and even insects and animal excrements [108a]. Hydrogen evolution was observed from all these unconventional feedstocks for long irradiation times under light from a source (Xe lamp) emitting mostly in the visible frequency. Production rates averaged over $10 \mathrm{~h}$ of reaction were in the $10^{1}-10^{2} \mu \mathrm{mol} \mathrm{g}_{\mathrm{cat}}{ }^{-1} \mathrm{~h}^{-1}$ range (Table 8), that is, approximately two orders of magnitude lower than for pure oxygenated molecules, as expected given the high chemical complexity of raw biomass. They further extended the validity of the method to other complex feedstocks such as bee wax, sweet potato, grass, wood, seaweed, lignin or pitch [108b, 214]. Furthermore, the same authors demonstrated that photoreforming of fossil fuels (coal, oil sands, hydrocarbons) [161b-d, 214] or even synthetic chemicals and polymers [108a, 214] is also possible in aqueous suspensions containing $\mathrm{Pt} / \mathrm{TiO}_{2}$. 
The valorisation of agricultural waste by transformation into fuels is an attractive opportunity. In this line, photocatalytic production of $\mathrm{H}_{2}$ from residual crop by-products rich in lignocellulosic biomass has been taken into consideration by Speltini et al., who demonstrated hydrogen production from rice husks or alfalfa stems [313b]. They found that alfalfa stems produced significant amounts of $\mathrm{H}_{2}(24 \mu \mathrm{mol}$ at $4 \mathrm{~h}$ irradiation time) after minor pretreatment (drying, milling and sieving). On the contrary, identical pretreatment largely suppressed $\mathrm{H}_{2}$ evolution in the case of rice husks, a fact ascribed by the authors to exceeding light absorption or scattering by the resulting suspension. However, yields comparable to those for alfalfa were obtained for untreated rice husks under both UVA and natural sunlight (Table 8), rendering the process truly renewable in terms of feedstock and energy source. Humic substances, a rather undefined blend composed of decaying organic matter, have also served as the substrate for photocatalytic $\mathrm{H}_{2}$ production under simulated solar light [362].

\subsection{Photocatalytic decontamination of waste streams with concomitant $\mathrm{H}_{2}$ production}

One of the most appealing and industrially relevant applications of photocatalysis is the removal of air and water pollutants by taking advantage of the oxidising and anti-microbial action of semiconductor materials, especially $\mathrm{TiO}_{2}$ [66a]. Several researchers independently noted that, although these systems perform very efficiently in aerobic conditions, the same processes may also take place in the absence of oxygen. In aqueous media, water itself acts as an oxidising agent which is in turn reduced to hydrogen gas [9, 38a, 98, 108a, 363]. Therefore, this approach, in essence happening via photoreforming of the organic matter present as pollutants can be beneficial in multiple senses, since decontamination is carried out with concomitant production of a clean fuel, and relying solely on a renewable, virtually inextinguishable, source of energy as is sunlight.

Wastewater streams in the food industry are interesting options for valorisation since they are likely to contain significant amounts of poly-functionalised organic substances. Regarding photocatalytic $\mathrm{H}_{2}$ production, this concept has been proven for olive mill wastewater by Spetlini and co-workers [321]. They demonstrated relatively high $\mathrm{H}_{2}$ production rates on $\mathrm{Pt} / \mathrm{TiO}_{2}$ under UVA irradiation; unfortunately, simulated solar light resulted in low activities range (Table 8), which could however be improved by addition of an organic photosensitiser [321]. Not surprisingly, oil deposits on the catalyst surface led to fouling, and degreasing of the feed was required for sustained activity.

Malato and co-workers went a step further and proved the direct use of municipal wastewater in a real solar photoreactor at Plataforma Solar de Almería (Spain) [318]. The engineering and design included a tubular reactor ensuring a turbulent flow; this favoured light harvest from the suspended photocatalyst (optimum concentration around $0.01 \mathrm{~g} \mathrm{~L}^{-1}$ ) and prevented sedimentation. Parabolic 
concentrators were employed in order to focus sunlight irradiation and maximise it around the entire surface of the perimeter. Several other parameters, such as temperature, $\mathrm{pH}$ and characteristics of the reactor wall were examined. Regarding the latter topic, borosilicate glass was suggested as the optimum candidate given its reasonable transparency $(>285 \mathrm{~nm})$ and low cost. Other researchers have demonstrated the feasibility of human urine for the solar production of hydrogen in photoelectrochemical cells [364].

As a model waste generated in livestock farms, swine sludge has been considered for combined photocatalytic $\mathrm{H}_{2}$ production and remediation. This by-product is a challenging and complex matrix containing oxygenated and nitrogenated (among other) substances. Kida et al. performed a previous digestion in hot compressed water, after which simple oxygenates - such as methanol and formic acid-were identified and claimed to become the reactive substrates for $\mathrm{H}_{2}$ production on a $\mathrm{LaMnO}_{3} / \mathrm{CdS}$ photocatalyst under mainly visible light irradiation [365]. Speltini and co-workers opted for a simple centrifuge pretreatment to remove water-insoluble impurities, yielding a clear matrix having nearly neutral $\mathrm{pH}$ (7.9) and a relatively elevated organic content (chemical oxygen demand $=46.4 \mathrm{~g} \mathrm{~L}^{-1}$ ) [325]. Production of $\mathrm{H}_{2}$ was surprisingly higher under simulated sunlight than under UVA irradiation (Table 8), a phenomenon which was hypothesised as an effect of in situ sensitisation by coloured sludge components.

To summarise, several issues are worth being stressed about the direct photocatalytic production of $\mathrm{H}_{2}$ from raw biomass or wastewaters in aqueous suspensions. Although efficiencies are lower than for pure substrates, the strategy is successful for a diverse range of feedstocks and can be powered by direct sunlight. Therefore, this technology would be applicable not only to biorefineries, but also to municipal, industrial or farming wastewater treatment plants. Overall, the opportunity of valorising low-value agricultural by-products or even polluted streams with concomitant production of $\mathrm{H}_{2}$ as a clean fuel appears promising. 


\begin{tabular}{|c|c|c|c|c|c|c|c|c|c|c|c|c|}
\hline \multirow[b]{2}{*}{ photocatalyst } & \multirow[b]{2}{*}{ reaction medium } & \multirow[b]{2}{*}{ light source } & \multirow{2}{*}{$\begin{array}{l}P / \mathrm{W} \\
\left(\mathrm{I} / \mathrm{mW} \mathrm{cm}^{-2}\right)\end{array}$} & \multirow[b]{2}{*}{$T /{ }^{\circ} \mathrm{C}$} & \multicolumn{6}{|c|}{ production rates $/ \mu \mathrm{mol} \mathrm{g}_{\mathrm{cat}}{ }^{-1} \mathrm{~h}^{-1}$} & \multirow{2}{*}{$\begin{array}{l}- \\
\Phi_{a} / \%\end{array}$} & \multirow{2}{*}{$\begin{array}{l}- \\
\text { reference }\end{array}$} \\
\hline & & & & & $\mathrm{H}_{2}$ & $\mathrm{CO}_{2}$ & $\mathrm{HCHO}$ & $\mathrm{HCO}_{2} \mathrm{H}$ & $\mathrm{CH}_{4}$ & $\mathrm{CO}$ & & \\
\hline $\mathrm{TiO}_{2}$ (mesoporous) & $\mathrm{H}_{2} \mathrm{O} / \mathrm{MeOH}(l, 9: 1)$ & $\mathrm{Xe}(>320 \mathrm{~nm})$ & 300 & - & 85 & - & - & - & - & - & - & {$[47 \mathrm{a}]$} \\
\hline $\mathrm{Pt}(4 \%) / \mathrm{TiO}_{2}$ & $\mathrm{H}_{2} \mathrm{O} / \mathrm{MeOH}(l, 1: 1)$ & $\mathrm{Xe}(>320 \mathrm{~nm})$ & 500 & - & 3000 & - & - & - & - & - & - & {$[366]$} \\
\hline $\mathrm{Pt}(2 \%) / \mathrm{TiO}_{2}$ & $\mathrm{MeOH}(v)$ & $\mathrm{Xe}(350-400 \mathrm{~nm})$ & 500 & 25 & 6 & 0.03 & - & - & - & - & - & {$[80 \mathrm{f}]$} \\
\hline $\mathrm{Pt}(2 \%) / \mathrm{TiO}_{2}$ & $\mathrm{MeOH} / \mathrm{H}_{2} \mathrm{O}(v, 6.7: 1)$ & $\mathrm{Xe}(350-400 \mathrm{~nm})$ & 500 & 25 & 24 & 4.17 & - & - & - & - & - & {$[80 \mathrm{f}]$} \\
\hline $\mathrm{Pt}(4.85 \%) / /^{\mathrm{P} 25} \mathrm{TiO}_{2}$ & $\mathrm{MeOH}(l)$ & $\mathrm{Hg}$ & 125 & - & 4524 & - & - & - & - & - & - & {$[43]$} \\
\hline $\mathrm{Pt}(4.85 \%) / /^{\mathrm{P} 25} \mathrm{TiO}_{2}$ & $\mathrm{H}_{2} \mathrm{O} / \mathrm{MeOH}(l, 1: 1)$ & $\mathrm{Hg}$ & 125 & - & 3810 & - & - & - & - & - & - & [43] \\
\hline $\mathrm{Pt}(5 \%)+\mathrm{TiO}_{2}$ (rutile) & $\mathrm{H}_{2} \mathrm{O} / \mathrm{MeOH}(l, 1: 1)$ & $\mathrm{Hg}$ & 100 & - & 2022 & - & - & - & - & - & - & {$[132]$} \\
\hline $\mathrm{Pt}(5 \%)+\mathrm{TiO}_{2}($ rutile $)$ & $\mathrm{MeOH}(a q, 0.1 \mathrm{M})$ & $\mathrm{Hg}$ & 500 & - & 40 & - & 28 & - & - & - & - & {$[59]$} \\
\hline $\mathrm{Pt}(0.6 \%) / \mathrm{SiO}_{2}+{ }^{\mathrm{P} 25} \mathrm{TiO}_{2}$ & $\mathrm{MeOH}(a q, 0.5 \mathrm{M})$ & $\mathrm{Xe}(350-420 \mathrm{~nm})$ & 900 & - & 1072 & - & - & - & - & - & - & [367] \\
\hline $\mathrm{Pt}(0.5 \%) /^{\mathrm{P} 25} \mathrm{TiO}_{2}$ & $\mathrm{MeOH}(l)$ & $\mathrm{Hg}(>366 \mathrm{~nm})$ & 250 & 20 & - & - & 2267 & - & - & - & - & {$[70]$} \\
\hline $\mathrm{Pt}(0.1 \%) /{ }^{\mathrm{P} 25} \mathrm{TiO}_{2}$ & $\mathrm{H}_{2} \mathrm{O} / \mathrm{MeOH}(l, 9: 1)$ & $\mathrm{Hg}$ & 450 & - & 4000 & - & - & - & - & - & - & [208] \\
\hline $\mathrm{Pt}(0.5 \%) /{ }^{\mathrm{P} 25} \mathrm{TiO}_{2}$ & $\mathrm{MeOH}(a q, 0.85 \mathrm{mM})$ & solar simulator & 280 & 40 & 269 & 32 & - & - & - & - & - & [38a] \\
\hline $\mathrm{Pt}(0.5 \%) /{ }^{\mathrm{P} 25} \mathrm{TiO}_{2}$ & $\mathrm{MeOH}(a q, 1 \mathrm{M})$ & LED $(365 \mathrm{~nm})$ & (3) & - & 4005 & 248 & - & - & - & - & - & {$[38 \mathrm{c}]$} \\
\hline $\mathrm{Pt}(0.5 \%) /{ }^{\mathrm{P} 25} \mathrm{TiO}_{2}$ & $\mathrm{MeOH}(a q, 0.1 \mathrm{M})$ & LED (365 nm) & (3) & - & 2025 & - & - & - & - & - & - & {$[38 \mathrm{c}]$} \\
\hline $\mathrm{Pt}(1 \%) / \mathrm{TiO}_{2}$ (anatase) & $\mathrm{MeOH}(l)$ & $\mathrm{Hg}$ & 100 & 33 & 2356 & - & - & - & - & - & - & {$[368]$} \\
\hline $\mathrm{Pt}(0.3 \%) /{ }^{\mathrm{P} 25} \mathrm{TiO}_{2}$ & $\mathrm{H}_{2} \mathrm{O} / \mathrm{MeOH}(l, 100: 3)$ & $\mathrm{Hg}$ & 450 & 60 & 25556 & - & - & - & - & - & - & [369] \\
\hline $\mathrm{Pt}(0.1 \%) /{ }^{\mathrm{P} 25} \mathrm{TiO}_{2}$ & $\mathrm{H}_{2} \mathrm{O} / \mathrm{MeOH}(l, 4: 1)$ & $\mathrm{Xe}$ & 300 & - & 1492 & - & - & - & - & - & - & {$[94 b]$} \\
\hline $\mathrm{Pt}(0.1 \%) / \mathrm{TiO}_{2}(74 \%$ rutile $)$ & $\mathrm{H}_{2} \mathrm{O} / \mathrm{MeOH}(l, 4: 1)$ & $\mathrm{Xe}$ & 300 & - & 7094 & - & - & - & - & - & - & [94b] \\
\hline $\mathrm{Pd}(0.5 \%) / \mathrm{TiO}_{2}$ & $\mathrm{H}_{2} \mathrm{O} / \mathrm{MeOH}(l, 1000: 1)$ & $\mathrm{Xe}$ & 400 & - & 555 & - & - & - & - & - & - & {$[346]$} \\
\hline $\mathrm{Pt} /$ blackTiO $_{2}$ & $\mathrm{H}_{2} \mathrm{O} / \mathrm{MeOH}(l, 1: 1)$ & solar & - & - & 10000 & - & - & - & - & - & - & {$[178 \mathrm{a}]$} \\
\hline $\mathrm{Pt}(0.3 \%) /{ }^{\mathrm{P} 25} \mathrm{TiO}_{2}$ & $\mathrm{H}_{2} \mathrm{O} / \mathrm{MeOH}(l, 3: 2)$ & $\mathrm{Hg}(300-400 \mathrm{~nm})$ & - & - & 5767 & - & - & - & - & - & - & {$[370]$} \\
\hline $17 \% \mathrm{Pt}+33 \%$ graphite-silica $+{ }^{\mathrm{P}^{2} 5} \mathrm{TiO}_{2}$ & $\mathrm{H}_{2} \mathrm{O} / \mathrm{MeOH}(l, 3: 2)$ & $\mathrm{Hg}(300-400 \mathrm{~nm})$ & - & - & 4800 & - & - & - & - & - & - & {$[370]$} \\
\hline $\mathrm{Pt}(0.5 \%) / \mathrm{SiO}_{2}-\mathrm{TiO}_{2}$ & $\mathrm{H}_{2} \mathrm{O} / \mathrm{MeOH}(l, 20: 3.7)$ & $\mathrm{Xe}$ & 200 & - & 13 & - & - & - & - & - & - & {$[371]$} \\
\hline $\mathrm{Pt}(0.5 \%) / \mathrm{TiO}_{2}$ & $\mathrm{H}_{2} \mathrm{O} / \mathrm{MeOH}(l, 4: 3)$ & $\mathrm{Hg}$ & 300 & - & 8560 & - & - & - & - & - & - & {$[120 \mathrm{~b}]$} \\
\hline $\mathrm{Pt}(0.5 \%) / \mathrm{TiO}_{2}$ & $\mathrm{H}_{2} \mathrm{O} / \mathrm{MeOH}(l, 4: 3)$ & $\mathrm{Hg}$ & 300 & - & 8800 & - & - & - & - & - & - & {$[372]$} \\
\hline $\mathrm{Pt}(0.6 \%) / \mathrm{TiO}_{2}$ (mesoporous) & $\mathrm{H}_{2} \mathrm{O} / \mathrm{MeOH}(l, 10: 1)$ & $\mathrm{Hg}(>320 \mathrm{~nm})$ & 300 & - & 6925 & - & - & - & - & - & - & {$[140 b]$} \\
\hline $\mathrm{Pt}(0.5 \%) / \mathrm{TiO}_{2}$ (porous) & $\mathrm{H}_{2} \mathrm{O} / \mathrm{MeOH}(l, 5: 1)$ & $\mathrm{Hg}$ & 500 & 30 & 13000 & - & - & - & - & - & - & [120a] \\
\hline $\mathrm{PtO}(1 \%) / \mathrm{TiO}_{2}$ (nanosheets) & $\mathrm{H}_{2} \mathrm{O} / \mathrm{MeOH}(l, 7: 3)$ & $\mathrm{Xe}$ & 300 & - & 4400 & - & - & - & - & - & - & {$[8 \mathrm{a}]$} \\
\hline $\mathrm{Pt}(0.5 \%) / \mathrm{TiO}_{2}$ (nanowires) & $\mathrm{H}_{2} \mathrm{O} / \mathrm{MeOH}(l, 9: 1)$ & $\mathrm{Hg}$ & 450 & r.t. & 162 & - & - & - & - & - & - & [113a] \\
\hline $\mathrm{Pt}(0.5 \%) / \mathrm{TiO}_{2}$ (anatase/brookite, $\left.72: 28\right)$ & $\mathrm{MeOH}(a q, 4.93 \mathrm{M})$ & $\mathrm{Xe}$ & 1000 & - & 4213 & - & - & - & - & - & - & {$[121 b]$} \\
\hline $\mathrm{Pt}(0.5 \%) / \mathrm{TiO}_{2}$ (brookite) & $\mathrm{MeOH}(a q, 4.93 \mathrm{M})$ & $\mathrm{Xe}$ & 1000 & - & 3973 & - & - & - & - & - & - & {$[121 b]$} \\
\hline $\mathrm{Pt}(0.1 \%) / \mathrm{TiO}_{2-\mathrm{x}}$ & $\mathrm{H}_{2} \mathrm{O} / \mathrm{MeOH}(l, 3: 1)$ & $\mathrm{Xe}(>400 \mathrm{~nm})$ & 300 & - & 50 & - & - & - & - & - & - & [179] \\
\hline $\mathrm{Pt}(0.4 \%) / \mathrm{TiO}_{2}$ (anatase/rutile 39:62) & $\mathrm{H}_{2} \mathrm{O} / \mathrm{MeOH}(l, 9: 1)$ & $\mathrm{Xe}(>320 \mathrm{~nm})$ & 300 & - & 4250 & - & - & - & - & - & - & {$[147]$} \\
\hline $\mathrm{Pt}(1 \%)+\mathrm{TiO}_{2}$ (nanofibers) & $\mathrm{H}_{2} \mathrm{O} / \mathrm{MeOH}(l, 9: 1)$ & $\mathrm{Xe}$ & 300 & - & 10860 & - & - & - & - & - & - & {$[128 \mathrm{~d}]$} \\
\hline $\mathrm{Pt} / \mathrm{TiO}_{2}$ (anatase) & $\mathrm{H}_{2} \mathrm{O} / \mathrm{MeOH}(l, 1: 1)$ & $\mathrm{Xe}$ & 300 & 25 & 16500 & - & - & - & - & - & - & [326] \\
\hline $\mathrm{Pt}(1 \%))^{225} \mathrm{TiO}_{2} / \mathrm{SiO}_{2}$ & $\mathrm{H}_{2} \mathrm{O} / \mathrm{MeOH}(v, 7: 3)$ & solar simulator & $(100)$ & 280 & 571000 & - & - & - & - & - & 78 & {$[181]$} \\
\hline$\left.\mathrm{Pt}(1 \%)\right|^{\mathrm{P25}} \mathrm{TiO}_{2} / \mathrm{SiO}_{2}$ & $\mathrm{H}_{2} \mathrm{O} / \mathrm{MeOH}(v, 7: 3)$ & solar simulator $(>420 \mathrm{~nm})$ & $(100)$ & 280 & 497000 & - & - & - & - & - & 65.7 & {$[181]$} \\
\hline $\mathrm{Pt}(0.3 \%) / \mathrm{Gd}(0.5 \%): \mathrm{TiO}_{2}$ & $\mathrm{H}_{2} \mathrm{O} / \mathrm{MeOH}(l, 50: 1)$ & $\mathrm{Hg}$ & 300 & - & 13200 & - & - & - & - & - & - & {$[373]$} \\
\hline $\mathrm{Pt}(0.3 \%) / \mathrm{Eu}(0.5 \%): \mathrm{TiO}_{2}$ & $\mathrm{H}_{2} \mathrm{O} / \mathrm{MeOH}(l, 50: 1)$ & $\mathrm{Hg}$ & 300 & - & 10800 & - & - & - & - & - & - & [373] \\
\hline $\mathrm{RuO}_{2} / \mathrm{Pt} / \mathrm{TiO}_{2}$ & $\mathrm{H}_{2} \mathrm{O} / \mathrm{MeOH}(l, 1: 1)$ & $\mathrm{Xe}$ & 500 & - & 1733 & - & - & - & - & - & - & {$[100 \mathrm{~b}]$} \\
\hline
\end{tabular}


$\mathrm{RuO}_{2} / \mathrm{Pt} / \mathrm{TiO}_{2}$

$\mathrm{Pt}(0.05 \%) /{ }^{\mathrm{P2} 2} \mathrm{TiO}_{2}$

$\mathrm{Pt}(0.5 \%) / \mathrm{TiO}_{2}$

$\mathrm{Pt}(2 \%) / \mathrm{TiO}_{2}$

$\mathrm{Pt}(2 \%) / \mathrm{TiO}_{2}$

$\mathrm{Pt}(2 \%) / \mathrm{TiO}_{2}$

$\mathrm{Rh}(2 \%) / \mathrm{TiO}_{2}$

$\mathrm{Pd}(2 \%) / \mathrm{TiO}_{2}$

$\mathrm{RuO}_{2}(2 \%) / \mathrm{TiO}_{2}$

$\mathrm{Ag}(2 \%) / \mathrm{TiO}_{2}$

$\mathrm{Pt}(1 \%) /\left(\mathrm{CNT}+\mathrm{TiO}_{2}\right)$

$\mathrm{Pd}(1 \%) /\left(\mathrm{CNT}+\mathrm{TiO}_{2}\right)$

$\mathrm{Pt}(0.5 \%) / \mathrm{TiO}_{2} / \mathrm{CNT}(10 \%)$ (nanofibers)

$\mathrm{Pt}(0.5 \%) / \mathrm{TiO}_{2}$

$\mathrm{Au}(1 \%) /{ }^{\mathrm{P} 25} \mathrm{TiO}_{2}$

$\mathrm{Pt}(0.3 \%) / \mathrm{TiO}_{2}$

$\mathrm{Au}(2 \%) / \mathrm{TiO}_{2}$

$\mathrm{Pd}(1 \%) / \mathrm{TiO}_{2}$

$\mathrm{Au}(1 \%) / \mathrm{TiO}_{2}$

$\mathrm{Pd}(0.5 \%) / \mathrm{TiO}_{2}$

$\mathrm{Pd}(0.5 \%) / \mathrm{TiO}_{2}$

$\mathrm{Au}(2 \%) / \mathrm{TiO}_{2}$

$\mathrm{Au}(0.43 \%) / /^{\mathrm{P25}} \mathrm{TiO}_{2}$

$\mathrm{Pd}(5 \%){ }^{\mathrm{P25}} \mathrm{TiO}_{2}$

$\mathrm{Pd}_{\text {shell }} \mathrm{Au}_{\text {core }}(1 \%) / /^{\mathrm{P} 25} \mathrm{TiO}_{2}$

$\mathrm{Pd}(0.5 \%))^{225} \mathrm{TiO}_{2}$

$\mathrm{Au}(0.5 \%) /{ }^{\mathrm{P} 25} \mathrm{TiO}_{2}$

$\mathrm{Au}(1 \%) / \mathrm{TiO}_{2}$

$\mathrm{Au}(1 \%) / /^{\mathrm{P25}} \mathrm{TiO}_{2}$

$\mathrm{Au}(1 \%) /{ }^{\mathrm{FP}} \mathrm{TiO}_{2}$

$\mathrm{Pt}(1 \%) /{ }^{\mathrm{P} 25} \mathrm{TiO}_{2}$

$\mathrm{Au}(1 \%) /{ }^{\mathrm{P} 25} \mathrm{TiO}_{2}$

$\mathrm{Ag}(1 \%) /{ }^{\mathrm{P} 25} \mathrm{TiO}_{2}$

${ }^{\mathrm{P} 25} \mathrm{TiO}_{2}$

$\mathrm{Au}(1 \%) / \mathrm{TiO}_{2}$ (anatase)

$\mathrm{Au}(1.5 \%) /{ }^{\mathrm{P} 25} \mathrm{TiO}_{2}$

$\mathrm{Au}(1.5 \%) / \mathrm{TiO}_{2}$ (anatase)

$\mathrm{Au}(1.5 \%) / \mathrm{TiO}_{2}$ (brookite)

$\mathrm{Au}(1.5 \%) / \mathrm{TiO}_{2}$ (rutile)

$\mathrm{Au}(\mathrm{Au} / \mathrm{Ti}=0.017) @ \mathrm{TiO}_{2}$ (nanotubes)

$\mathrm{Au}(\mathrm{Au} / \mathrm{Ti}=0.015) / \mathrm{TiO}_{2}$ (nanotubes)

$\mathrm{Ag}(0.5 \%) / \mathrm{TiO}_{2}$ (rutile)

$\mathrm{Ag}(0.5 \%) / \mathrm{TiO}_{2}$ (anatase)

\begin{tabular}{|c|c|}
\hline $\mathrm{H}_{2} \mathrm{O} / \mathrm{MeOH}(l, 1: 1)$ & $\mathrm{Hg}$ \\
\hline $\operatorname{MeOH}(a q, 5 \mathrm{M})$ & $\mathrm{Xe}$ \\
\hline $\mathrm{H}_{2} \mathrm{O} / \mathrm{MeOH}(l, 1: 1)$ & $\mathrm{Xe}$ \\
\hline $\mathrm{MeOH}(l)$ & $\mathrm{Xe}$ \\
\hline $\mathrm{H}_{2} \mathrm{O} / \mathrm{MeOH}(l, 9: 1)$ & $\mathrm{Xe}$ \\
\hline $\mathrm{MeOH}(l)$ & $\mathrm{Xe}$ \\
\hline $\mathrm{MeOH}(l)$ & $\mathrm{Xe}$ \\
\hline $\mathrm{MeOH}(l)$ & $\mathrm{Xe}$ \\
\hline $\mathrm{MeOH}(l)$ & $\mathrm{Xe}$ \\
\hline $\mathrm{MeOH}(l)$ & $\mathrm{Xe}$ \\
\hline $\mathrm{MeOH}(a q, 2.5 \mathrm{M})$ & $\mathrm{Hg}$ \\
\hline $\mathrm{MeOH}(a q, 2.5 \mathrm{M})$ & $\mathrm{Hg}$ \\
\hline $\mathrm{H}_{2} \mathrm{O} / \mathrm{MeOH}(l, 1: 1)$ & $\mathrm{Hg}$ \\
\hline $\mathrm{H}_{2} \mathrm{O} / \mathrm{MeOH}(v, 60: 40)$ & Fe-halogen-Hg \\
\hline $\mathrm{H}_{2} \mathrm{O} / \mathrm{MeOH}(v, 69: 31)$ & Fe-halogen-Hg \\
\hline $\mathrm{H}_{2} \mathrm{O} / \mathrm{MeOH}(l, 3: 1)$ & solar simulator \\
\hline $\mathrm{H}_{2} \mathrm{O} / \mathrm{MeOH}(l, 100: 1)$ & solar simulator \\
\hline $\mathrm{H}_{2} \mathrm{O} / \mathrm{MeOH}(l, 1000: 1)$ & solar \\
\hline $\mathrm{H}_{2} \mathrm{O} / \mathrm{MeOH}(l, 1000: 1)$ & solar \\
\hline $\mathrm{MeOH}(a q, 18.6 \mathrm{M})$ & $\mathrm{Xe}$ \\
\hline $\mathrm{H}_{2} \mathrm{O} / \mathrm{MeOH}(l, 1000: 1)$ & $\mathrm{Xe}$ \\
\hline $\mathrm{H}_{2} \mathrm{O} / \mathrm{MeOH}(l, 1000: 1)$ & $\mathrm{Xe}$ \\
\hline $\operatorname{MeOH}(a q, 1 \mathrm{M})$ & $\mathrm{Xe}$ \\
\hline $\mathrm{H}_{2} \mathrm{O} / \mathrm{MeOH}(l, 1: 1)$ & - \\
\hline $\mathrm{H}_{2} \mathrm{O} / \mathrm{MeOH}(1,3: 1)$ & UV LED (365 nm) \\
\hline $\mathrm{H}_{2} \mathrm{O} / \mathrm{MeOH}(l, 1000: 1)$ & $\mathrm{Xe}$ \\
\hline $\mathrm{H}_{2} \mathrm{O} / \mathrm{MeOH}(l, 1000: 1)$ & $\mathrm{Xe}$ \\
\hline $\mathrm{H}_{2} \mathrm{O} / \mathrm{MeOH}(100: 6)$ & $\mathrm{Hg}$ \\
\hline $\mathrm{H}_{2} \mathrm{O} / \mathrm{MeOH}(100: 6)$ & $\mathrm{Hg}$ \\
\hline $\mathrm{H} 2 \mathrm{O} / \mathrm{MeOH}(v)$ & $\mathrm{Hg}$ \\
\hline $\mathrm{MeOH} / \mathrm{H}_{2} \mathrm{O}(v, 2: 3)$ & $\mathrm{Hg}$ \\
\hline $\mathrm{MeOH} / \mathrm{H}_{2} \mathrm{O}(v, 2: 3)$ & $\mathrm{Hg}$ \\
\hline $\mathrm{MeOH} / \mathrm{H}_{2} \mathrm{O}(v, 2: 3)$ & $\mathrm{Hg}$ \\
\hline $\mathrm{MeOH} / \mathrm{H}_{2} \mathrm{O}(v, 2: 3)$ & $\mathrm{Hg}$ \\
\hline $\mathrm{H}_{2} \mathrm{O} / \mathrm{MeOH}(l, 1: 1)$ & $\mathrm{Xe}$ \\
\hline $\mathrm{H}_{2} \mathrm{O} / \mathrm{MeOH}(l, 9: 1)$ & UV $(365 \mathrm{~nm})$ \\
\hline $\mathrm{H}_{2} \mathrm{O} / \mathrm{MeOH}(l, 9: 1)$ & UV $(365 \mathrm{~nm})$ \\
\hline $\mathrm{H}_{2} \mathrm{O} / \mathrm{MeOH}(l, 9: 1)$ & UV $(365 \mathrm{~nm})$ \\
\hline $\mathrm{H}_{2} \mathrm{O} / \mathrm{MeOH}(l, 9: 1)$ & UV $(365 \mathrm{~nm})$ \\
\hline $\mathrm{H}_{2} \mathrm{O} / \mathrm{MeOH}(l, 10: 1)$ & $\mathrm{Xe}(>400 \mathrm{~nm})$ \\
\hline $\mathrm{H}_{2} \mathrm{O} / \mathrm{MeOH}(l, 10: 1)$ & $\mathrm{Xe}(>400 \mathrm{~nm})$ \\
\hline $\mathrm{H}_{2} \mathrm{O} / \mathrm{MeOH}(l, 1: 1)$ & UVA $(352 \mathrm{~nm})$ \\
\hline $\mathrm{H}_{2} \mathrm{O} / \mathrm{MeOH}(l, 1: 1)$ & UVA $(352 \mathrm{~nm})$ \\
\hline
\end{tabular}

500
300
450
450
450
450
450
450
450
450
150
150
200
-
-
$(30)$
$(30)$
280
280
400
400
400
300
-
$(60)$
400
400
250
250
250
250
250
250
250
300
$(6.5)$
$(6.5)$
$(6.5)$
$(6.5)$
$6 \times 6$

700

30000

5900

4500

25016

23229

27696

19655

18762

12508

1251

1380

40600

17500

15000

1080
7200

100

1700

465

169

383
2261

20529

486

347

7890

5400

10200

$18600 \quad 2880$

$13300 \quad 1610$

$1170 \quad 32$

720

8400

13500

8500

6700

900

482

223

357

222 [94c]

[374]

[108d]

[108d]

[108d]

[108d]

[108d]

[108d]

[108d]

[159a]

[159a]

[159b]

[77]

[77]

[133]

[133]

[264]

[264]

[57]

[90a]

[91b]

[94a]

[375]

[287]

[286]

[286]

[103]

[100c]

[100c]

[328]

[328]

[328]

[328]

[326]

- [115]

- [115]

- [115]

[115]

[122]

[122]

[112] 
$\mathrm{Au}(0.9 \%) /{ }^{\mathrm{P} 25} \mathrm{TiO}_{2}$

$\mathrm{Sn}(0.24 \%) / \mathrm{RuO}_{2}(0.68 \%) /^{\mathrm{P} 25} \mathrm{TiO}_{2}$

$\mathrm{Sn}(0.24 \%))^{\mathrm{P} 25} \mathrm{TiO}_{2}$

$\mathrm{Pt}(3 \%) / \mathrm{Sn}(1 \%) / \mathrm{TiO}_{2}$

$\mathrm{Pt}(3 \%) / \mathrm{Sn}(1 \%) / \mathrm{TiO}_{2}$

$\mathrm{Cu}(3 \% \mathrm{~mol} v$ s. $\mathrm{Ti}) / \mathrm{S}: \mathrm{TiO}_{2}$

$\mathrm{Cu}(3 \%$ mol vs. $\mathrm{Ti}) / \mathrm{TiO}_{2}$

$\mathrm{NiO}(1.5 \%) / \mathrm{TiO}_{2}$

$\mathrm{NiO}(0.5 \%) / \mathrm{TiO}^{2}$

$\mathrm{NiO}(0.5 \%) / \mathrm{TiO}_{2}$

$\mathrm{CuO}(1 \%)+{ }^{\mathrm{P} 25} \mathrm{TiO}_{2}$

$\mathrm{CuO}(0.5 \%)+\mathrm{Al}_{2} \mathrm{O}_{3}(0.3 \%)+{ }^{\mathrm{P} 25} \mathrm{TiO}_{2}$

$\mathrm{CuO}(9.1 \%) /{ }^{\mathrm{P} 25} \mathrm{TiO}_{2}$

$\mathrm{Fe}(20 \%) / \mathrm{TiO}_{2}$

$\mathrm{RGO} / \mathrm{TiO}_{2}$

$\mathrm{RGO}(16.7 \%) /{ }^{\mathrm{P} 25} \mathrm{TiO}_{2}$

graphite-silica $(50 \%)+{ }^{\mathrm{P} 25} \mathrm{TiO}_{2}$

$\mathrm{TiO}_{2-x}$

$\mathrm{ZnO}$

$\mathrm{Pt}(0.5 \%) / \mathrm{Cu}_{2} \mathrm{O}$

$\mathrm{Cu}_{2} \mathrm{O}$

$\mathrm{Pt}(0.5 \%) / \mathrm{SrTiO}_{3}$

$\mathrm{SrTiO}_{3}$

$\mathrm{Rh}-\mathrm{Cr} / \mathrm{N}: \mathrm{ZnGa}_{2} \mathrm{O}_{4}$

$\mathrm{K}_{4} \mathrm{Nb}_{6} \mathrm{O}_{7}$

$\mathrm{H}_{2.04} \mathrm{~K}_{1.96} \mathrm{Nb}_{6} \mathrm{O}_{17}$

$\mathrm{NiO}(0.1 \%) / \mathrm{K}_{4} \mathrm{Nb}_{6} \mathrm{O}_{17}$

$\mathrm{Pt}(0.1 \%) / \mathrm{SiO}_{2}$ (pillars)- $\mathrm{Ca}_{2} \mathrm{Nb}_{3} \mathrm{O}_{10}$

$\mathrm{Pt}(0.5 \%) / \mathrm{CdS}$

$\mathrm{Pt}(3,8 \%) / \mathrm{CdS}$

$\mathrm{Pt}(5 \%) / \mathrm{CdS}$

$\mathrm{Pt}(5 \%) / \mathrm{CdS}$

$\mathrm{MoS}_{2}(0.2 \%) / \mathrm{CdS}$

$\mathrm{Pt}(0.2 \%) / \mathrm{CdS}$

CdSe-CdS (nanorods)-P

$\mathrm{Ru}(5 \%) / \mathrm{ZnS} / \mathrm{CdS}(\mathrm{Cd} / \mathrm{Zn}=4)$

$\mathrm{ZnS}$

$\mathrm{CuO}(\text { layer })^{[a]}$

$\mathrm{Cu}_{2} \mathrm{O}$ (layer) ${ }^{[a]}$

$\mathrm{Pt}+\mathrm{UiO}-66\left(\mathrm{NH}_{2}\right)^{[b]}$

UiO-66 $\left(\mathrm{NH}_{2}\right)^{[b]}$

$\mathrm{P}$ :graphene

N(5.4\%):graphene

$\begin{array}{ll}\mathrm{H}_{2} \mathrm{O} / \mathrm{MeOH}(l, 3: 1) & \text { solar simulator } \\ \mathrm{H}_{2} \mathrm{O} / \mathrm{MeOH}(l, 33: 1) & \mathrm{Hg} \\ \mathrm{H}_{2} \mathrm{O} / \mathrm{MeOH}(l, 33: 1) & \mathrm{Hg} \\ \mathrm{H}_{2} \mathrm{O} / \mathrm{MeOH}(l, 33: 1) & \mathrm{Hg} \\ \mathrm{H}_{2} \mathrm{O} / \mathrm{MeOH}(l, 33: 1) & \mathrm{Hg} \\ \mathrm{H}_{2} \mathrm{O} / \mathrm{MeOH}(l, 9: 1) & \mathrm{Hg} \\ \mathrm{H}_{2} \mathrm{O} / \mathrm{MeOH}(l, 9: 1) & \mathrm{Hg} \\ \mathrm{H}_{2} \mathrm{O} / \mathrm{MeOH}(l, 30: 1) & \mathrm{Hg} \\ \mathrm{MeOH}(a q, 0.03 \mathrm{M}) & \mathrm{UV}(300-400 \mathrm{~nm}) \\ \mathrm{MeOH}(a q, 0.03 \mathrm{M}) & \mathrm{UV}(300-400 \mathrm{~nm}) \\ \mathrm{H}_{2} \mathrm{O} / \mathrm{MeOH}(l, 9: 1) & \text { black light } \\ \mathrm{H}_{2} \mathrm{O} / \mathrm{MeOH}(l, 9: 1) & \text { black light } \\ \mathrm{H}_{2} \mathrm{O} / \mathrm{MeOH}(l, 9: 1) & \mathrm{Hg} \\ \mathrm{MeOH}(a q, 1 \mathrm{M}) & \mathrm{Hg} \\ \mathrm{H}_{2} \mathrm{O} / \mathrm{MeOH}(l, 3: 1) & \mathrm{Xe}-\mathrm{Hg} \\ \mathrm{H}_{2} \mathrm{O} / \mathrm{MeOH}(l, 4: 1) & \mathrm{Xe} \\ \mathrm{H}_{2} \mathrm{O} / \mathrm{MeOH}(l, 3: 2) & \mathrm{UV} \\ \mathrm{H}_{2} \mathrm{O} / \mathrm{MeOH}(l, 4: 1) & \mathrm{Xe}(>420 \mathrm{~nm}) \\ \mathrm{H}_{2} \mathrm{O} / \mathrm{MeOH}(l, 9: 1) & \mathrm{Xe} \\ \mathrm{MeOH}(a q, 2.5 \mathrm{M}) & \mathrm{halogen}(>420 \mathrm{~nm}) \\ \mathrm{MeOH}(a q, 2.5 \mathrm{M}) & \text { halogen }(>420 \mathrm{~nm}) \\ \mathrm{H}_{2} \mathrm{O} / \mathrm{MeOH}(1: 1) & \mathrm{Hg} \\ \mathrm{MeOH}(a q, 1 \mathrm{M}) & \mathrm{Hg} \\ \mathrm{H}_{2} \mathrm{O} / \mathrm{MeOH}(l, 9: 1) & \mathrm{Hg} \\ \mathrm{H}_{2} \mathrm{O} / \mathrm{MeOH}(l, 4: 1) & \mathrm{Xe} \\ \mathrm{H}_{2} \mathrm{O} / \mathrm{MeOH}(l, 4: 1) & \mathrm{Xe} \\ \mathrm{H}_{2} \mathrm{O} / \mathrm{MeOH}(l, 30: 1) & \mathrm{Hg} \\ \mathrm{H}_{2} \mathrm{O} / \mathrm{MeOH}(l, 9: 1) & \mathrm{Hg} \\ \mathrm{MeOH}(a q, 1 \mathrm{M}) & \mathrm{Xe}(>450 \mathrm{~nm}) \\ \mathrm{MeOH}(a q, 12 \mathrm{M}) & \mathrm{Hg}(>400 \mathrm{~nm}) \\ \mathrm{H}_{2} \mathrm{O} / \mathrm{MeOH}(l, 100: 0.17) & \mathrm{Xe} \\ \mathrm{H}_{2} \mathrm{O} / \mathrm{MeOH}(l, 100: 0.17) & \mathrm{Xe}(>430 \mathrm{~nm}) \\ \mathrm{H}_{2} \mathrm{O} / \mathrm{MeOH}(l, 9: 1) & \mathrm{Xe}(>420 \mathrm{~nm}) \\ \mathrm{H}_{2} \mathrm{O} / \mathrm{MeOH}(l, 9: 1) & \mathrm{Xe}(>420 \mathrm{~nm}) \\ \mathrm{H}_{2} \mathrm{O} / \mathrm{MeOH}(l, 50: 1) & \mathrm{Xe} \\ \mathrm{H}_{2} \mathrm{O} / \mathrm{MeOH}(l, 9: 1) & \mathrm{Xe}(>420 \mathrm{~nm}) \\ \mathrm{H}_{2} \mathrm{O} / \mathrm{MeOH}(l, 7: 1) & \mathrm{Hg}(>290 \mathrm{~nm}) \\ \mathrm{H}_{2} \mathrm{O} / \mathrm{MeOH}(l, 1: 1) & \text { vis } \\ \mathrm{H}_{2} \mathrm{O} / \mathrm{MeOH}(l, 1: 1) & \text { vis } \\ \mathrm{H}_{2} \mathrm{O} \mathrm{MeOH}(l, 3: 1) & \mathrm{Xe}-\mathrm{Hg} \\ & \end{array}$

25 [269a]

$\begin{array}{llll}70 & 500 & 2.8 & {[283]}\end{array}$

$22-[283]$

- [376]

[376]

[120d]

[120d]

[377]

[76]

[76]

[378]

[378]

[272d]

[379]

[302]

[158a]

[180]

[189]

[193]

[193]

[201a]

[201d]

[381]

[207a]

[207b]

[207c]

[208]

[213a]

[218]

[214]

[214]

[292]

[292]

[382]

[224]

[231b]

[194]

[194]

[253]

$3.5 \quad$ [253]

[244]

[243] 
$\mathrm{Pt}(0.5 \%) / g-\mathrm{C}_{3} \mathrm{~N}_{4}$

$\mathrm{H}_{2} \mathrm{O} / \mathrm{MeOH}(l, 9: 1)$

$\mathrm{Xe}(>420 \mathrm{~nm})$

300

30

[247]

${ }^{[a]}$ Formed by chemical vapour deposition. ${ }^{[b]} \mathrm{UiO}-66\left(\mathrm{NH}_{2}\right)$ : poly- $\mathrm{Zr}$-(2-amino)benzenedicarboxylate metal organic framework.

Table 3. Ethanol Photoreforming Data

\begin{tabular}{|c|c|c|c|c|c|c|c|c|c|c|c|c|c|}
\hline \multirow[b]{2}{*}{ photocatalyst } & \multirow[b]{2}{*}{ reaction medium } & \multirow[b]{2}{*}{ light source } & \multirow{2}{*}{$\begin{array}{l}P / \mathrm{W} \\
\left(I / \mathrm{mW} \mathrm{cm}^{-2}\right)\end{array}$} & \multirow[b]{2}{*}{$T /{ }^{\circ} \mathrm{C}$} & \multicolumn{7}{|c|}{ production rates $/ \mu \mathrm{mol} \mathrm{g}_{\mathrm{cat}}{ }^{-1} \mathrm{~h}^{-1}$} & \multirow[b]{2}{*}{$\Phi_{\mathrm{a}} / \%$} & \multirow[b]{2}{*}{ reference } \\
\hline & & & & & $\mathrm{H}_{2}$ & $\mathrm{CO}_{2}$ & $\mathrm{CH}_{3} \mathrm{CHO}$ & $\mathrm{CH}_{4}$ & $\mathrm{CO}$ & $\mathrm{C}_{2} \mathrm{H}_{4}$ & $\mathrm{C}_{2} \mathrm{H}_{6}$ & & \\
\hline${ }^{\mathrm{P} 25} \mathrm{TiO}_{2}$ & $\mathrm{EtOH}(l)$ & - & 10 & - & - & - & 467 & 1.3 & - & - & - & - & {$[100 \mathrm{~d}]$} \\
\hline $\mathrm{Ti}_{2} \mathrm{O}_{3}$ & $\operatorname{EtOH}(l)$ & - & 10 & - & 0.04 & 0.10 & 10 & - & - & - & - & - & {$[100 \mathrm{~d}]$} \\
\hline $\mathrm{TiO}$ & $\operatorname{EtOH}(l)$ & - & 10 & - & 0.17 & - & $65^{[a]}$ & 0.9 & - & - & - & - & {$[100 \mathrm{~d}]$} \\
\hline $\mathrm{Bi}(0.5 \% \mathrm{~mol}): \mathrm{TiO}_{2}$ & $\mathrm{H}_{2} \mathrm{O} / \mathrm{EtOH}(l, 1: 1)$ & UVB (312 nm) & $6 \times 8$ & - & 800 & - & - & - & - & - & - & - & {$[383]$} \\
\hline $\mathrm{Pt}(4.85 \%) /{ }^{\mathrm{P} 25} \mathrm{TiO}_{2}$ & $\mathrm{EtOH}(l)$ & $\mathrm{Hg}$ & 125 & - & 2022 & 18 & $1846^{[a]}$ & - & - & - & - & - & [43] \\
\hline $\mathrm{Pt}(4 \%) / \mathrm{TiO}_{2}$ & $\operatorname{EtOH}(a q, 1.7 \%$ w/v $)$ & $\mathrm{Xe}$ & 500 & - & 1693 & - & - & - & - & - & - & - & {$[108 \mathrm{~b}]$} \\
\hline $\mathrm{Pt}(0.5 \%) / \mathrm{TiO}_{2}$ & $\mathrm{EtOH}(a q, 1 \mathrm{M})$ & solar & 450 & 40 & 5475 & - & - & - & - & - & - & - & [9] \\
\hline $\mathrm{Pt}(0.5 \%) / \mathrm{TiO}_{2}$ & $\mathrm{EtOH}(a q, 0.86 \mathrm{mM})$ & solar & 280 & 80 & 690 & 60 & - & - & - & - & - & - & [38a] \\
\hline $\mathrm{Pt}(0.5 \%) / \mathrm{TiO}_{2}$ & $\mathrm{EtOH}(a q, 8.91 \mathrm{mM})$ & solar & 280 & 40 & 4950 & - & - & - & - & - & - & - & [38a] \\
\hline $\mathrm{Pt}(0.5 \%) / \mathrm{TiO}_{2}$ & $\operatorname{EtOH}(a q, 0.28 \mathrm{mM})$ & $\mathrm{Hg}$ & 450 & 40 & 22500 & - & - & - & - & - & - & - & [38a] \\
\hline $\mathrm{Pt}(0.4 \%) / \mathrm{TiO}_{2}$ (anatase) & $\operatorname{EtOH}(a q, 0.05 \mathrm{M})$ & $\mathrm{Xe}$ & 150 & - & 4750 & - & - & - & - & - & - & - & {$[384]$} \\
\hline $\mathrm{Pt}(0.5 \%) / \mathrm{TiO}_{2}$ & $\operatorname{EtOH}(l)$ & $\mathrm{Hg}(366 \mathrm{~nm})$ & 250 & 20 & - & - & 2267 & - & - & - & - & - & {$[70]$} \\
\hline $\mathrm{Pt}(0.5 \%) / \mathrm{TiO}_{2}$ & $\mathrm{H}_{2} \mathrm{O} / \mathrm{EtOH}(l, 4: 3)$ & $\mathrm{Hg}$ & 300 & - & 8600 & - & - & - & - & - & - & - & [372] \\
\hline $\mathrm{Pt}(5 \%) / \mathrm{TiO}_{2}$ (anatase) & $\operatorname{EtOH}(l)$ & $\mathrm{Hg}$ & 400 & r.t. & 168 & - & 225 & - & - & - & - & - & [349a] \\
\hline $\mathrm{Pt}(5 \%) / \mathrm{TiO}_{2}$ (anatase) & $\operatorname{EtOH}(a q, 0.1 \mathrm{M})$ & $\mathrm{Hg}$ & 500 & - & 40 & - & 34 & - & - & - & - & - & [59] \\
\hline $\mathrm{Pt}(1 \%$, atom $) / \mathrm{TiO}_{2}$ (anatase) & $\mathrm{H}_{2} \mathrm{O} / \mathrm{EtOH}(l, 20: 1)$ & $\mathrm{UV}(>365 \mathrm{~nm})$ & (2) & - & 3630 & - & - & - & - & - & - & - & {$[152]$} \\
\hline $\mathrm{Pt}(0.2 \%) / \mathrm{TiO}_{2}$ (anatase/brookite) & $\mathrm{EtOH}(l, 96 \%)$ & solar simulator & 150 & 25 & 930 & - & - & - & - & - & - & - & {$[124]$} \\
\hline $\mathrm{Pt}(4 \%) / \mathrm{TiO}_{2}$ & $\mathrm{H}_{2} \mathrm{O} / \mathrm{EtOH}(l, 1: 1)$ & $\mathrm{Xe}(>320 \mathrm{~nm})$ & 500 & - & 2667 & - & - & - & - & - & - & - & {$[366]$} \\
\hline $\mathrm{Pt}(2 \%) / \mathrm{TiO}_{2}$ & $\operatorname{EtOH}(l)$ & $\mathrm{Xe}$ & 450 & - & 16081 & - & - & - & - & - & - & - & {$[108 \mathrm{~d}]$} \\
\hline $\mathrm{Pt}(0.3 \%))^{\mathrm{P25}} \mathrm{TiO}_{2}$ & $\operatorname{EtOH}(a q, 1 \mathrm{mM})$ & $\mathrm{Hg}(>320 \mathrm{~nm})$ & 250 & - & 800 & - & - & - & - & - & - & - & {$[356]$} \\
\hline $\mathrm{Pt}(0.5 \%) / \mathrm{TiO}_{2}$ & $\mathrm{H}_{2} \mathrm{O} / \mathrm{EtOH}(l, 57: 43 \%)$ & $\mathrm{Hg}$ & 300 & - & 7667 & - & - & - & - & - & - & - & {$[385]$} \\
\hline $\mathrm{Pt}(2.1 \%) / \mathrm{TiO}_{2}$ & $\operatorname{EtOH}(a q, 7.34 \mathrm{M})$ & UV $300-400 \mathrm{~nm}$ & $12 \times 15$ & - & 11074 & 15 & $11^{[b]}$ & 79 & - & - & 123 & - & [330] \\
\hline $\mathrm{Pt}(0.6 \%) / \mathrm{TiO}_{2}$ (mesoporous) & $\mathrm{H}_{2} \mathrm{O} / \mathrm{EtOH}(l, 10: 1)$ & $\mathrm{Hg}(>320 \mathrm{~nm})$ & 300 & - & 5615 & - & - & - & - & - & - & - & {$[140 \mathrm{~b}]$} \\
\hline $\mathrm{Pt}(0.5 \%) / \mathrm{TiO}_{2}$ (porous) & $\mathrm{H}_{2} \mathrm{O} / \mathrm{EtOH}(l, 5: 1)$ & $\mathrm{Hg}$ & 500 & 30 & 8075 & - & - & - & - & - & - & - & [120a] \\
\hline $\mathrm{Pt}(1 \%) / \mathrm{TiO}_{2}$ (aerogel) & $\mathrm{H}_{2} \mathrm{O} / \mathrm{EtOH}(l, 99: 1)$ & UV & 400 & - & 7200 & - & - & - & - & - & - & - & {$[141]$} \\
\hline $\mathrm{Pt}(2 \%) / \mathrm{F}: \mathrm{TiO}_{2}$ (nanosheets) & $\mathrm{H}_{2} \mathrm{O} / \mathrm{EtOH}(l, 3: 1)$ & $\mathrm{Xe}$ & (20) & - & 16675 & - & - & - & - & - & - & - & [121a] \\
\hline $\mathrm{Pt}(0.5 \%) / \mathrm{TiO}_{2}$ (nanotubes) & $\mathrm{H}_{2} \mathrm{O} / \mathrm{EtOH}(l, 9: 1)$ & Xe (solar simulator) & 350 & - & 47800 & - & - & - & - & - & - & - & [143b] \\
\hline $\mathrm{Pt}(1 \%) / \mathrm{TiO}_{2}$ (nanofibers) & $\operatorname{EtOH}(l)$ & UVA (352 nm) & $2 \times 15$ & r.t. & 23850 & - & - & - & - & - & - & - & {$[128 \mathrm{c}]$} \\
\hline $\mathrm{Pt}(1 \%))^{/ 25} \mathrm{TiO}_{2}$ & $\operatorname{EtOH}(l)$ & UVA $(352 \mathrm{~nm})$ & $2 \times 15$ & r.t. & 22050 & - & - & - & - & - & - & - & {$[128 \mathrm{c}]$} \\
\hline $\mathrm{Pt}(1 \%) / \mathrm{TiO}_{2}$ (nanotubes) & $\operatorname{EtOH}(l)$ & $\mathrm{Hg}(315-400 \mathrm{~nm})$ & $2 \times 15$ & r.t. & 34750 & - & - & - & - & - & - & - & [113b] \\
\hline $\mathrm{Pt}(1 \%))^{\mid 225} \mathrm{TiO}_{2}$ & $\operatorname{EtOH}(l)$ & $\mathrm{Hg}(315-400 \mathrm{~nm})$ & $2 \times 15$ & r.t. & 27850 & - & - & - & - & - & - & - & {$[113 b]$} \\
\hline $\mathrm{Pt}(1 \%) /{ }^{\mathrm{P} 25} \mathrm{TiO}_{2}$ & $\operatorname{EtOH}(l)$ & UVA (352 nm) & $2 \times 15$ & r.t. & 22000 & - & - & - & - & - & - & - & {$[128 \mathrm{~b}]$} \\
\hline $\mathrm{Pt}(1 \%) / \mathrm{TiO}_{2}$ (nanofibers) & $\mathrm{EtOH}(l)$ & UVA (352 nm) & $2 \times 15$ & r.t. & 23800 & - & - & - & - & - & - & - & {$[128 b]$} \\
\hline $\mathrm{TiO}_{2}$ (nanofibers) & $\mathrm{H}_{2} \mathrm{O} / \mathrm{EtOH}(l, 3: 1 \mathrm{~mol})$ & UVB $(312 \mathrm{~nm})$ & $6 \times 3.15$ & - & 300 & - & - & - & - & - & - & - & {$[129]$} \\
\hline $\mathrm{Pt}(1 \%) / \mathrm{TiO}_{2}$ (nanofibers) & $\mathrm{H}_{2} \mathrm{O} / \mathrm{EtOH}(l, 3: 1 \mathrm{~mol})$ & UVB $(312 \mathrm{~nm})$ & $6 \times 3.15$ & - & 22500 & - & - & - & - & - & - & - & [129] \\
\hline $\mathrm{Pd}(1 \%) / \mathrm{TiO}_{2}$ (nanofibers) & $\mathrm{H}_{2} \mathrm{O} / \mathrm{EtOH}(l, 3: 1 \mathrm{~mol})$ & UVB (312 nm) & $6 \times 3.15$ & - & 15300 & - & - & - & - & - & - & - & [129] \\
\hline
\end{tabular}


$\mathrm{Pt} / \mathrm{TiO}_{2}$ (layer)

$\mathrm{Pt} / \mathrm{TiO}_{2}$ (layer)

$\mathrm{Pt} / \mathrm{TiO}_{2}$ (layer)

$\mathrm{Pt}(0.5 \%) /(\mathrm{S}-\mathrm{Eu}): \mathrm{TiO}_{2}$

$\mathrm{Pt}(0.5 \%) / \mathrm{S}: \mathrm{TiO}_{2}$

$\mathrm{Pt}(0.5 \%) /{ }^{\mathrm{P} 25} \mathrm{TiO}_{2}$

$\mathrm{Pt}(0.3 \%) / \mathrm{Gd}: \mathrm{TiO}_{2}$

$\mathrm{Pt}(0.5 \%) / \mathrm{S}: \mathrm{TiO}_{2}$

$\mathrm{Pt}(0.5 \%) / \mathrm{N}: \mathrm{TiO}_{2}$

$\mathrm{Pt}(0.5 \%) / \mathrm{TiO}_{2}$

$\mathrm{Pd}(0.5 \%) / \mathrm{TiO}_{2}$

$\mathrm{Pd}(2 \%))^{\mathrm{P25}} \mathrm{TiO}_{2}$

$\mathrm{Pt}(0.5 \%) /{ }^{\mathrm{P} 25} \mathrm{TiO}_{2}$

$\mathrm{Au}(1 \%) / \mathrm{TiO}_{2}$

$\mathrm{Pt}(1 \%) / \mathrm{TiO}_{2}$

$\mathrm{Au}(2 \%) /{ }^{\mathrm{P} 25} \mathrm{TiO}_{2}$

$\mathrm{Pt}(1 \%) /{ }^{\mathrm{P} 25} \mathrm{TiO}_{2}$

${ }^{\mathrm{P} 25} \mathrm{TiO}_{2}$

$\mathrm{TiO}_{2}$ (anatase from $\mathrm{P} 25$ )

$\mathrm{TiO}_{2}$ (rutile from $\mathrm{P} 25$ )

$\mathrm{Au}(1 \%) /{ }^{\mathrm{P} 25} \mathrm{TiO}_{2}$

$\mathrm{Au}(3 \%) / \mathrm{TiO}_{2}$ (anatase from $\mathrm{P} 25$ )

$\mathrm{Au}(3 \%) / \mathrm{TiO}_{2}$ (rutile from $\mathrm{P} 25$ )

$\mathrm{Au}(1 \%) / \mathrm{TiO}_{2}$

$\mathrm{Au}(1 \%) / \mathrm{TiO}_{2}$

$\mathrm{Au}(0.2 \%$ atom $) /{ }^{225} \mathrm{TiO}_{2}$

${ }^{\mathrm{P} 25} \mathrm{TiO}_{2}$

$\mathrm{Au}(1.5 \%) /{ }^{\mathrm{P} 25} \mathrm{TiO}_{2}$

$\mathrm{Au}(1.5 \%) / \mathrm{TiO}_{2}$ (anatase)

$\mathrm{Au}(1.5 \%) / \mathrm{TiO}_{2}$ (brookite)

$\mathrm{Au}(1.5 \%) / \mathrm{TiO}_{2}$ (rutile)

$\mathrm{Au}(0.5 \%) / \mathrm{H}_{2} \mathrm{Ti}_{3} \mathrm{O}_{7}$ (nanotubes)

$\mathrm{Au}(0.5 \%) / \mathrm{TiO}_{2}$ (rutile)

$\mathrm{Au}(0.5 \%) / \mathrm{TiO}_{2}$ (anatase nanotubes)

$\mathrm{TiO}_{2}$ (anatase nanotubes)

$\mathrm{Au}(1.5 \%){ }^{\mathrm{P25}} \mathrm{TiO}_{2}$

$\mathrm{Au}(1 \%) /{ }^{\mathrm{P} 25} \mathrm{TiO}_{2}$

$\mathrm{Pd}(1 \%) /{ }^{\mathrm{P25}} \mathrm{TiO}_{2}$

$\mathrm{Au}_{3} \mathrm{Pd}(1 \%) /{ }^{\mathrm{P} 25} \mathrm{TiO}_{2}$

$\mathrm{Pd}_{3} \mathrm{Au}(1 \%) /{ }^{\mathrm{P} 25} \mathrm{TiO}_{2}$

$\mathrm{Au}_{\text {shell }} \mathrm{Pd}_{\text {core }}(1 \%) /{ }^{\mathrm{P} 25} \mathrm{TiO}_{2}$

$\mathrm{Pd}_{\text {shell }} \mathrm{Au}_{\text {core }}(1 \%) /{ }^{\mathrm{P} 25} \mathrm{TiO}_{2}$

$\mathrm{Au}(2 \%) / \mathrm{TiO}_{2}$ (macroporous)
$\mathrm{H}_{2} \mathrm{O} / \mathrm{EtOH}(l, 9: 1)$

$\mathrm{H}_{2} \mathrm{O} / \mathrm{EtOH}(l, 1: 1)$

$\mathrm{H}_{2} \mathrm{O} / \mathrm{EtOH}(l, 1: 1)$

$\mathrm{H}_{2} \mathrm{O} / \mathrm{EtOH}(l, 99: 1)$

$\mathrm{H}_{2} \mathrm{O} / \mathrm{EtOH}(l, 99: 1)$

$\mathrm{H}_{2} \mathrm{O} / \mathrm{EtOH}(l, 99: 1)$

$\mathrm{H}_{2} \mathrm{O} / \mathrm{EtOH}(l, 50: 1)$

$\mathrm{H}_{2} \mathrm{O} / \mathrm{EtOH}(l)$

$\mathrm{H}_{2} \mathrm{O} / \mathrm{EtOH}(l)$

$\mathrm{H}_{2} \mathrm{O} / \mathrm{EtOH}(l)$

$\mathrm{H}_{2} \mathrm{O} / \mathrm{EtOH}(l, 1000: 1)$

$\mathrm{H}_{2} \mathrm{O} / \mathrm{EtOH}(l, 1: 4)$

$\mathrm{H}_{2} \mathrm{O} / \mathrm{EtOH}(l, 1: 4)$

$\mathrm{EtOH}(a q, 5 \mathrm{M})$

$\mathrm{EtOH}(a q, 5 \mathrm{M})$

$\mathrm{H}_{2} \mathrm{O} / \mathrm{EtOH}(l, 1: 4)$

$\mathrm{H}_{2} \mathrm{O} / \mathrm{EtOH}(l, 1: 4)$

$\mathrm{H}_{2} \mathrm{O} / \mathrm{EtOH}(l, 1: 4)$

$\mathrm{H}_{2} \mathrm{O} / \mathrm{EtOH}(l, 1: 4)$

$\mathrm{H}_{2} \mathrm{O} / \mathrm{EtOH}(l, 1: 4)$

$\mathrm{H}_{2} \mathrm{O} / \mathrm{EtOH}(l, 1: 4)$

$\mathrm{H}_{2} \mathrm{O} / \mathrm{EtOH}(l, 1: 4)$

$\mathrm{H}_{2} \mathrm{O} / \mathrm{EtOH}(l, 1: 4)$

$\mathrm{EtOH}(l)$

$\mathrm{EtOH}(l)$

$\mathrm{H}_{2} \mathrm{O} / \mathrm{EtOH}(l, 19: 1)$

$\mathrm{H}_{2} \mathrm{O} / \mathrm{EtOH}(l, 9: 1)$

$\mathrm{H}_{2} \mathrm{O} / \mathrm{EtOH}(l, 9: 1)$

$\mathrm{H}_{2} \mathrm{O} / \mathrm{EtOH}(l, 9: 1)$

$\mathrm{H}_{2} \mathrm{O} / \mathrm{EtOH}(l, 9: 1)$

$\mathrm{H}_{2} \mathrm{O} / \mathrm{EtOH}(l, 9: 1)$

$\mathrm{H}_{2} \mathrm{O} / \mathrm{EtOH}(l, 9: 1)$

$\mathrm{H}_{2} \mathrm{O} / \mathrm{EtOH}(l, 9: 1)$

$\mathrm{H}_{2} \mathrm{O} / \mathrm{EtOH}(l, 1: 4)$

$\mathrm{H}_{2} \mathrm{O} / \mathrm{EtOH}(l, 9: 1)$

$\mathrm{H}_{2} \mathrm{O} / \mathrm{EtOH}(l, 9: 1)$

$\mathrm{H}_{2} \mathrm{O} / \mathrm{EtOH}(l, 3: 1)$

$\mathrm{H}_{2} \mathrm{O} / \mathrm{EtOH}(l, 3: 1)$

$\mathrm{H}_{2} \mathrm{O} / \mathrm{EtOH}(l, 3: 1)$

$\mathrm{H}_{2} \mathrm{O} / \mathrm{EtOH}(l, 3: 1)$

$\mathrm{H}_{2} \mathrm{O} / \mathrm{EtOH}(l, 3: 1)$

$\mathrm{H}_{2} \mathrm{O} / \mathrm{EtOH}(l, 3: 1)$

$\mathrm{H}_{2} \mathrm{O} / \mathrm{EtOH}(l, 199: 1)$ black light (300-400 nm)

black light

black light

UV

UV

$\mathrm{Hg}$

$\mathrm{Xe}$

$\mathrm{Xe}$

$\mathrm{Xe}$

$\mathrm{Xe}$

UV (365 nm)

UV $(365 \mathrm{~nm})$

$\mathrm{Hg}(276-342 \mathrm{~nm})$

$\mathrm{Hg}(276-342 \mathrm{~nm})$

UV (365 nm)

UV $(365 \mathrm{~nm})$

UV $(365 \mathrm{~nm})$

UV $(365 \mathrm{~nm})$

UV $(365 \mathrm{~nm})$

UV $(365 \mathrm{~nm})$

UV $(365 \mathrm{~nm})$

UV $(365 \mathrm{~nm})$

$\mathrm{Hg}$

solar simulator

$\mathrm{Hg}>360 \mathrm{~nm}$

UV $(365 \mathrm{~nm})$

UV $(365 \mathrm{~nm})$

UV $(365 \mathrm{~nm})$

UV (365 nm)

UV $(365 \mathrm{~nm})$

UV (365 nm)

UV (365 nm)

UV (365 nm)

UV (365 nm)

UV $(365 \mathrm{~nm})$

UV LED (365 nm)

UV LED (365 nm)

UV LED $(365 \mathrm{~nm})$

UV LED (365 nm)

UV LED $(365 \mathrm{~nm})$

UV LED (365 nm)

sunlight
(0.80)

$\times 4$

$(80)$

(80)

(80)

300

(340)

(340)

(340)

400

$(6.5)$

(6.5)

(30)

(30)

(6.5)

(6.5)

(6.5)

(6.5)

(6.5)

(6.5)

(6.5)

125

(100)

(4-5)

(6.5)

(6.5)

(6.5)

(6.5)

(6.5)

(6.5)

$(6.5)$

(60)

(60)

(60)

(60)

(60)

(1)

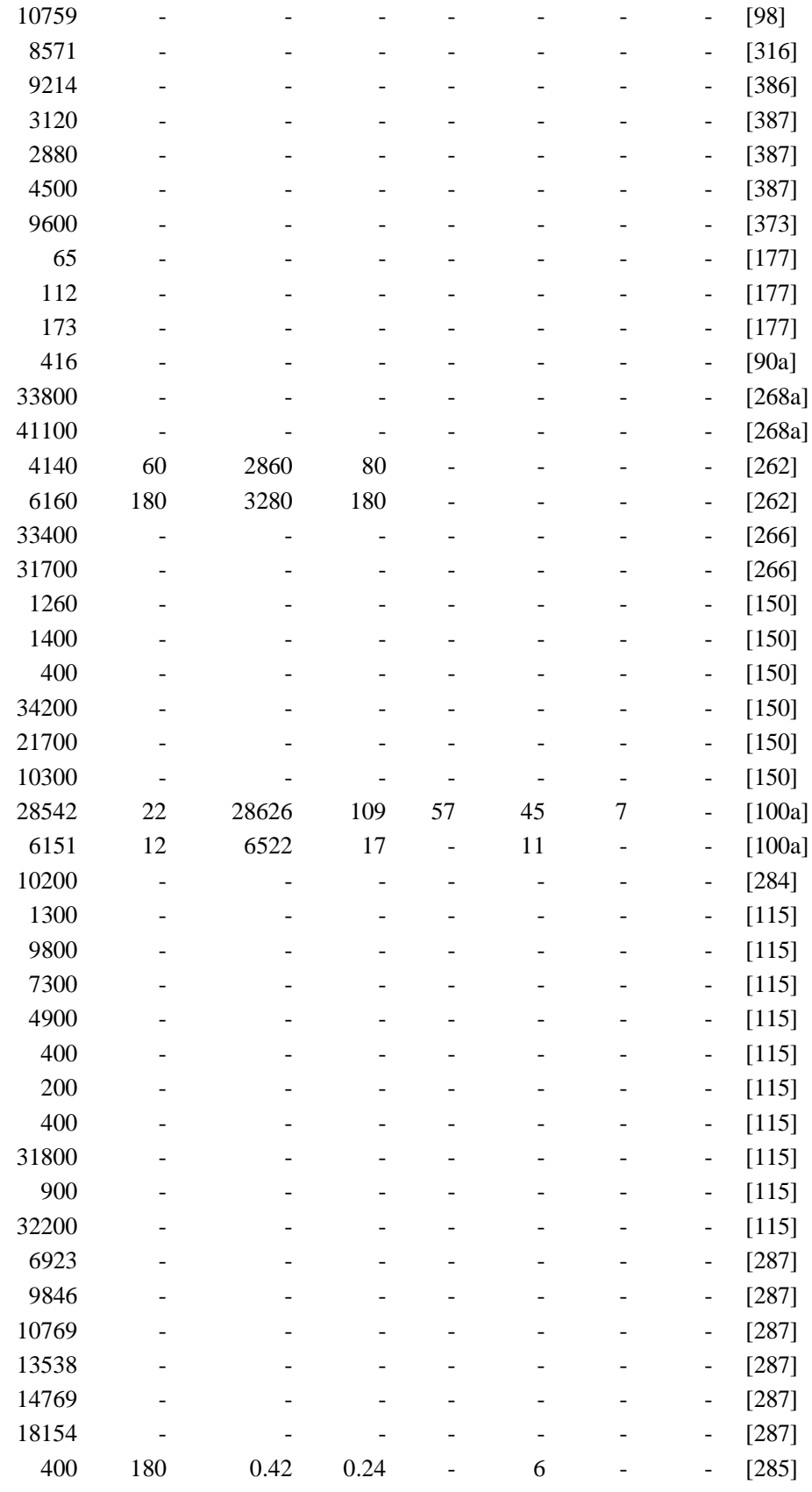


$\mathrm{Au}(0.5 \%)-\mathrm{Pd}(0.5 \%) / \mathrm{TiO}_{2}$ (macroporous)

$\mathrm{Au}(0.5 \%)-\mathrm{Pd}(0.5 \%) / \mathrm{TiO}_{2}$

$\mathrm{H}_{2} \mathrm{O} / \mathrm{EtOH}(l, 199: 1)$

sunlight

$\mathrm{Au}(0.5 \%)-\mathrm{Pd}(0.5 \%) / \mathrm{TiO}_{2}$

$\mathrm{H}_{2} \mathrm{O} / \mathrm{EtOH}(l, 1: 1)$

$\mathrm{Hg}$

$\mathrm{Au}(4 \%) / \mathrm{TiO}_{2}$

$\mathrm{Au}(2 \%) / \mathrm{TiO}_{2}$

$\mathrm{Au}(4.0 \%) / \mathrm{TiO}_{2}$ (anatase)

$\mathrm{AuPd} / \mathrm{TiO}_{2}$

$\mathrm{AuPd} / \mathrm{TiO}_{2}$

$\mathrm{Au}(1.5 \%) /{ }^{\mathrm{P} 25} \mathrm{TiO}_{2}$

$\mathrm{TiO}_{2}$ (rutile)

$\mathrm{RuO}_{2}(9 \%) / \mathrm{TiO}_{2}$ (rutile)

$\mathrm{Pd}(9 \%) / \mathrm{TiO}_{2}$ (rutile)

$\mathrm{Pt}(9 \%) / \mathrm{TiO}_{2}$ (rutile)

$\mathrm{Rh}(1 \%) / \mathrm{TiO}_{2}$

$\mathrm{Pd}(1 \%) / \mathrm{TiO}_{2}$

$\mathrm{Pt}(1 \%) / \mathrm{TiO}_{2}$

$\mathrm{Ag}(2 \%) / /^{225} \mathrm{TiO}_{2}$

$\mathrm{Au}(1 \%) / \mathrm{TiO}_{2} /$ cordierite(monolith)

$\mathrm{Au}(1 \%) / \mathrm{TiO}_{2}$

$\mathrm{Au}(1 \%) / \mathrm{TiO}_{2} /$ cordierite(monolith)

$\mathrm{Au}_{3} \mathrm{Cu}(1 \%) / \mathrm{TiO}_{2} /$ cordierite

$\mathrm{Au}_{3} \mathrm{Cu}(1 \%) / \mathrm{TiO}_{2} /$ cordierite

${ }^{\mathrm{P} 25} \mathrm{TiO}_{2}$ (layer)

$\mathrm{Au}(0.5 \%))^{\mathrm{P} 25} \mathrm{TiO}_{2}$ (layer)

$\mathrm{Cu}(0.5 \%){ }^{\mathrm{P} 25} \mathrm{TiO}_{2}$ (layer)

$\mathrm{Cu}(1 \%) /{ }^{\mathrm{P} 25} \mathrm{TiO}_{2}$

$\mathrm{Pt}(1.5 \%) /{ }^{\mathrm{P} 25} \mathrm{TiO}_{2}$

$\mathrm{CuO}(1.25 \%) /{ }^{\mathrm{P} 25} \mathrm{TiO}_{2}$

$\mathrm{CuO}_{x}(2.5 \%) @ \mathrm{TiO}_{2}$

$\mathrm{CuO}_{x}(1 \%) / \mathrm{TiO}_{2}$

$\mathrm{CuO}_{x}(1 \%) / \mathrm{TiO}_{2}$

$\mathrm{Cu}(1.0 \%) / \mathrm{TiO}_{2}$ (layer)

$\mathrm{Cu}(1.0 \%) / \mathrm{TiO}_{2}$

$\mathrm{Cu}(1.0 \%) / \mathrm{TiO}_{2}$

$\mathrm{CuO}(\mathrm{Ti} / \mathrm{Cu}=9) / \mathrm{C}($ fiber, $1 \%) /{ }^{225} \mathrm{TiO}_{2}$

$\mathrm{H}_{2} \mathrm{O} / \mathrm{EtOH}(l, 1: 1)$

Xe (solar)

$\mathrm{EtOH}(l)$

UV $(350 \mathrm{~nm})$

$\mathrm{EtOH}(l)$

$\mathrm{H}_{2} \mathrm{O} / \mathrm{EtOH}(l, 1: 1)$

$\mathrm{H}_{2} \mathrm{O} / \mathrm{EtOH}(a q, 5 \mathrm{M}) \quad \mathrm{Xe} 250-380 \mathrm{~nm}$

$\mathrm{H}_{2} \mathrm{O} / \mathrm{EtOH}(a q, 5 \mathrm{M}) \quad \mathrm{Xe} 385-740 \mathrm{~nm}$

black light

$\mathrm{H}_{2} \mathrm{O} / \mathrm{EtOH}(1,1: 1) \quad \mathrm{Xe}$

$\mathrm{H}_{2} \mathrm{O} / \mathrm{EtOH}(1,1: 1) \quad \mathrm{Xe}$

$\mathrm{H}_{2} \mathrm{O} / \mathrm{EtOH}(1,1: 1) \quad \mathrm{Xe}$

$\mathrm{H}_{2} \mathrm{O} / \mathrm{EtOH}(1,1: 1) \quad \mathrm{Xe}$

$\mathrm{EtOH}(l) \quad \mathrm{Hg}$

$\mathrm{EtOH}(l)$

$\mathrm{EtOH}(l) \quad \mathrm{Hg}$

$\mathrm{H}_{2} \mathrm{O} / \mathrm{EtOH}(l, 20: 1) \quad \mathrm{UV}(>365 \mathrm{~nm})$

$\mathrm{H}_{2} \mathrm{O} / \mathrm{EtOH}(l, 1: 1) \quad$ fiber optics LED $(365 \mathrm{~nm})$

$\mathrm{H}_{2} \mathrm{O} / \mathrm{EtOH}(l, 1: 1) \quad$ fiber optics LED (365 nm)

$\mathrm{H}_{2} \mathrm{O} / \mathrm{EtOH}(l, 1: 1) \quad$ fiber optics LED $(365 \mathrm{~nm})$

fiber optics LED (365 nm)

fiber optics LED (365 nm)

$\mathrm{H}_{2} \mathrm{O} / \mathrm{EtOH}(v, 99: 1)$

$\mathrm{H}_{2} \mathrm{O} / \mathrm{EtOH}(v, 19: 1.5)$

$\mathrm{H}_{2} \mathrm{O} / \mathrm{EtOH}(v, 19: 1.5) \quad \mathrm{Xe}$

$\mathrm{H}_{2} \mathrm{O} / \mathrm{EtOH}(v, 19: 1.5) \quad \mathrm{X}$

$\mathrm{H}_{2} \mathrm{O} / \mathrm{EtOH}(l, 3: 17) \quad \mathrm{Hg}$

$\mathrm{H}_{2} \mathrm{O} / \mathrm{EtOH}(l, 1: 4) \quad \mathrm{UV}(365 \mathrm{~nm})$

$\mathrm{H}_{2} \mathrm{O} / \mathrm{EtOH}(l, 1: 4) \quad \mathrm{UV}(365 \mathrm{~nm})$

$\mathrm{H}_{2} \mathrm{O} / \mathrm{EtOH}(l, 1: 1) \quad \mathrm{Hg}$

$\mathrm{H}_{2} \mathrm{O} / \mathrm{EtOH}(l, 1: 1) \quad \mathrm{Hg}$

$\mathrm{H}_{2} \mathrm{O} / \mathrm{EtOH}(l, 1: 1) \quad$ solar simulator

$\mathrm{H}_{2} \mathrm{O} / \mathrm{EtOH}(v, 19: 1.5) \quad$ solar simulator

$\mathrm{H}_{2} \mathrm{O} / \mathrm{EtOH}(l, 1: 1) \quad \mathrm{Hg}$

$\mathrm{H}_{2} \mathrm{O} / \mathrm{EtOH}(l, 1: 1) \quad \mathrm{Hg}$

$\mathrm{H}_{2} \mathrm{O} / \mathrm{EtOH}(l, 9: 1) \quad \mathrm{Xe}$ (> 320 nm filter)

$\mathrm{H}_{2} \mathrm{O} / \mathrm{EtOH}(l, 1: 4) \quad \mathrm{UV}$

$\mathrm{H}_{2} \mathrm{O} / \mathrm{EtOH}(l, 1: 19) \quad \mathrm{UV}$

$\mathrm{H}_{2} \mathrm{O} / \mathrm{EtOH}(l, 1: 4) \quad$ UV

$\mathrm{H}_{2} \mathrm{O} / \mathrm{EtOH}(l, 1: 1) \quad \mathrm{Xe}$

$\mathrm{H}_{2} \mathrm{O} / \mathrm{EtOH}(l, 1: 1) \quad \mathrm{Xe}$

$\mathrm{H}_{2} \mathrm{O} / \mathrm{EtOH}(l, 1: 1) \quad \mathrm{Xe}$

$\mathrm{H}_{2} \mathrm{O} / \mathrm{EtOH}(l, 1: 1) \quad \mathrm{Xe}$

$\mathrm{H}_{2} \mathrm{O} / \mathrm{EtOH}(l, 1: 1) \quad \mathrm{Xe}$

\begin{tabular}{|c|c|c|c|c|c|c|c|c|c|c|}
\hline (1) & - & 600 & - & - & - & - & - & - & - & [285] \\
\hline 125 & 20 & 7500 & - & - & - & - & - & - & - & [100f] \\
\hline 150 & 25 & 1600 & - & - & - & - & - & - & - & [100f $]$ \\
\hline $6 \times 15$ & - & 6930 & - & - & - & - & - & - & - & [114] \\
\hline $6 \times 15$ & - & 5357 & - & - & - & - & - & - & - & [342] \\
\hline (10) & - & 1.7 & - & - & - & - & - & - & - & [388] \\
\hline (12.7) & - & 2670 & - & - & - & - & - & - & - & [389] \\
\hline (151) & - & 2570 & - & - & - & - & - & - & - & [389] \\
\hline (7) & - & 6000 & - & - & - & - & - & - & - & [22] \\
\hline 500 & - & 8 & - & - & - & - & - & - & - & [390] \\
\hline 500 & - & 263 & - & - & - & - & - & - & - & [390] \\
\hline 500 & - & 767 & - & - & - & - & - & - & - & [390] \\
\hline 500 & - & 1880 & - & - & - & - & - & - & - & [390] \\
\hline 100 & 33 & 480 & - & - & - & - & - & - & - & [368] \\
\hline 100 & 77 & 3532 & - & - & - & - & - & - & - & {$[368]$} \\
\hline 100 & 71 & 3480 & - & - & - & - & - & - & - & [368] \\
\hline (6.5) & - & 3740 & - & - & - & - & - & - & - & [391] \\
\hline$(0.2)$ & 25 & 948 & 54 & - & - & - & - & - & - & [329] \\
\hline$(0.2)$ & 25 & 186 & - & - & - & - & - & - & - & [329] \\
\hline$(0.2)$ & 25 & 912 & - & - & - & - & - & - & 38.0 & [392] \\
\hline (2.6) & 25 & 4150 & - & 3800 & - & - & - & - & - & [288] \\
\hline (2.6) & 25 & 2250 & 300 & 1350 & - & - & - & - & - & [288] \\
\hline (100) & - & 532 & 21 & 484 & 24 & 33 & - & - & - & [268b] \\
\hline (100) & - & 2125 & 78 & 1812 & 84 & 118 & - & - & - & [268b] \\
\hline (100) & - & 1862 & 55 & 1625 & 70 & 98 & - & - & - & [268b] \\
\hline 200 & r.t. & 9830 & - & - & - & - & - & - & - & [327] \\
\hline (6.5) & - & 29500 & - & - & - & - & - & - & - & [273] \\
\hline (6.5) & - & 20300 & - & - & - & - & - & - & - & [273] \\
\hline 125 & 20 & 1400 & - & - & - & - & - & - & - & [272a] \\
\hline 125 & - & 1700 & - & 250 & - & - & 80 & - & - & [277] \\
\hline 150 & - & 800 & - & - & - & - & - & - & - & [277] \\
\hline 300 & - & 1950 & - & 1000 & 67 & 83 & - & - & - & [120c] \\
\hline 125 & - & 833 & 20 & 240 & 20 & - & - & 80 & - & [120c] \\
\hline 125 & - & 592 & 20 & 160 & 15 & - & 15 & 50 & - & {$[120 \mathrm{c}]$} \\
\hline 300 & - & 2000 & - & - & - & - & - & - & - & [393] \\
\hline (6.5) & - & 1200 & - & - & - & - & - & - & - & [278] \\
\hline (6.5) & - & 24000 & - & - & - & - & - & - & - & [278] \\
\hline (6.5) & - & 32400 & - & - & - & - & - & - & - & [278] \\
\hline 500 & - & 1600 & - & - & - & - & - & - & - & [394] \\
\hline 500 & - & 830 & - & - & - & - & - & - & - & [394] \\
\hline 500 & - & 610 & - & - & - & - & - & - & - & [394] \\
\hline 500 & - & 290 & - & - & - & - & - & - & - & [394] \\
\hline 500 & - & 37 & - & - & - & - & - & - & - & [394] \\
\hline
\end{tabular}


$\mathrm{Ni} / \mathrm{TiO}_{2}$ (mesoporous)

$\mathrm{Cu} / \mathrm{TiO}_{2}$ (mesoporous)

$\mathrm{Ag} / \mathrm{TiO}_{2}$ (mesoporous)

$\mathrm{Fe}(20 \%) / \mathrm{TiO}_{2}$

$\mathrm{Fe}(5.0 \%)-\mathrm{Ni}(4.0 \%)-\mathrm{TiO}_{2}$

$\mathrm{Ag}-\mathrm{Fe}-\mathrm{Ni} / \mathrm{TiO}_{2}$

$\mathrm{Co}(0.4 \%) /{ }^{\mathrm{P} 25} \mathrm{TiO}_{2}$

${ }^{\mathrm{P} 25} \mathrm{TiO}_{2}$

$\mathrm{Sn}(0.24 \%) / \mathrm{RuO}_{2}(0.68 \%) /{ }^{\mathrm{P} 25} \mathrm{TiO}_{2}$

$\mathrm{Sn}(0.24 \%) / /^{\mathrm{P25}} \mathrm{TiO}_{2}$

$\mathrm{TiO}_{2}$ (anatase)

$\mathrm{Sn}(1 \%) / \mathrm{TiO}_{2}$ (anatase)

$\mathrm{Pt}(3 \%) / \mathrm{TiO}_{2}$ (anatase)

$\mathrm{Pt}(3 \%) / \mathrm{Sn}(1 \%) / \mathrm{TiO}_{2}$ (anatase)

$\mathrm{H}_{3} \mathrm{PMo}_{12} \mathrm{O}_{40} / \mathrm{TiO}_{2}(8 \%) / \mathrm{Co}-Y^{[c]}$

$\mathrm{H}_{3} \mathrm{PW}_{12} \mathrm{O}_{40} / \mathrm{TiO}_{2}(10 \%) / \mathrm{Co}-\mathrm{Na}-Y^{[c]}$

$\mathrm{TiO}_{2} /\left[\mathrm{MoS}_{2}(95 \%) / \mathrm{RGO}(5 \%)\right](0.5 \%)$

$\mathrm{TiO}_{2} /[\mathrm{RGO}(10 \%)](0.5 \%)$

$\mathrm{CuInS}_{2}(2.5 \%){ }^{\mid 25} \mathrm{TiO}_{2}$

$\mathrm{RGO}(16.7 \%) /{ }^{\mathrm{P} 25} \mathrm{TiO}_{2}$

$\mathrm{Pt}(0.5 \%) / \mathrm{Cu}_{2} \mathrm{O}$

$\mathrm{Cu}_{2} \mathrm{O}$

$\mathrm{Sn}$ (II): $\mathrm{SnO}_{2}$

$\mathrm{N}: \mathrm{TiO}_{2} / \mathrm{N}: \mathrm{In}_{2} \mathrm{O}_{3}$

$\varepsilon-\mathrm{Fe}_{2} \mathrm{O}_{3}$ (layer)

$\mathrm{Ag} / \varepsilon-\mathrm{Fe}_{2} \mathrm{O}_{3}$ (layer)

$\mathrm{Au} / \varepsilon-\mathrm{Fe}_{2} \mathrm{O}_{3}$ (layer)

$\alpha-\mathrm{Fe}_{2} \mathrm{O}_{3}$ (layer)

$\beta$ - $\mathrm{Fe}_{2} \mathrm{O}_{3}$ (layer)

$\varepsilon-\mathrm{Fe}_{2} \mathrm{O}_{3}$ (layer)

$\mathrm{F}: \mathrm{Co}_{3} \mathrm{O}_{4}$ (layer)

$\mathrm{F}: \mathrm{Co}_{3} \mathrm{O}_{4}$ (layer)

$\mathrm{CuO} / \mathrm{ZnO}$ (layer)

$\mathrm{VO}_{2}$ (layer)

$\mathrm{SrTiO}_{3}$

$\mathrm{Pt}(1 \%) / \mathrm{CaTiO}_{3}$

$\mathrm{Pt}(1 \%) / \mathrm{CaTi}_{0.93} \mathrm{Zr}_{0.07} \mathrm{O}_{3}$

$\mathrm{Pt}(0.5 \%) / \mathrm{SrTiO}_{3}$

$\mathrm{Rh}(0.5 \%) / \mathrm{SrTiO}_{3}$

${ }^{\mathrm{IM}} \mathrm{Pt}(0.5 \%) / \mathrm{SrTiO}_{3}$

$\mathrm{Sr}_{2 / 3} \mathrm{Zn}_{1 / 3} \mathrm{TiO}_{3}$

$\mathrm{Ba}_{5 / 6} \mathrm{Zn}_{1 / 6} \mathrm{TiO}_{3}$

$\mathrm{Pt}(0.1 \%) / \mathrm{SiO}_{2}$ (pillars)- $\mathrm{Ca}_{2} \mathrm{Nb}_{3} \mathrm{O}_{10}$
$\mathrm{H}_{2} \mathrm{O} / \mathrm{EtOH}(l, 1: 19)$

$\mathrm{H}_{2} \mathrm{O} / \mathrm{EtOH}(l, 1: 19)$

$\mathrm{H}_{2} \mathrm{O} / \mathrm{EtOH}(l, 1: 19)$

$\mathrm{EtOH}(a q, 1 \mathrm{M})$

$\mathrm{H}_{2} \mathrm{O} / \mathrm{EtOH}(l, 9: 1)$

$\mathrm{H}_{2} \mathrm{O} / \mathrm{EtOH}(l, 9: 1)$

$\mathrm{H}_{2} \mathrm{O} / \mathrm{EtOH}(l, 4: 1)$

$\mathrm{H}_{2} \mathrm{O} / \mathrm{EtOH}(l, 33: 1)$

$\mathrm{H}_{2} \mathrm{O} / \mathrm{EtOH}(l, 33: 1)$

$\mathrm{H}_{2} \mathrm{O} / \mathrm{EtOH}(l, 33: 1)$

$\mathrm{H}_{2} \mathrm{O} / \mathrm{EtOH}(l, 33: 1)$

$\mathrm{H}_{2} \mathrm{O} / \mathrm{EtOH}(l, 33: 1)$

$\mathrm{H}_{2} \mathrm{O} / \mathrm{EtOH}(l, 33: 1)$

$\mathrm{H}_{2} \mathrm{O} / \mathrm{EtOH}(l, 33: 1)$

$\mathrm{H}_{2} \mathrm{O} / \mathrm{EtOH}(l, 4.8 \%)$

$\mathrm{H}_{2} \mathrm{O} / \mathrm{EtOH}(l, 1: 20)$

$\mathrm{H}_{2} \mathrm{O} / \mathrm{EtOH}(l, 3: 1)$

$\mathrm{H}_{2} \mathrm{O} / \mathrm{EtOH}(l, 3: 1)$

$\mathrm{H}_{2} \mathrm{O} / \mathrm{EtOH}(l, 4: 1)$

$\mathrm{H}_{2} \mathrm{O} / \mathrm{EtOH}(l, 4: 1)$

$\operatorname{EtOH}(a q, 2.5 \mathrm{M})^{[e]}$

$\mathrm{EtOH}(a q, 2.5 \mathrm{M})^{[e]}$

$\mathrm{H}_{2} \mathrm{O} / \mathrm{EtOH}(l, 3.75 \%)$

$\mathrm{H}_{2} \mathrm{O} / \mathrm{EtOH}(l, 4: 1)$

$\mathrm{EtOH}(a q, 1.0 \mathrm{M})$

$\operatorname{EtOH}(a q, 1.0 \mathrm{M})$

$\mathrm{EtOH}(a q, 1.0 \mathrm{M})$

$\mathrm{EtOH}(a q, 8.5 \mathrm{M})$

$\mathrm{EtOH}(a q, 8.5 \mathrm{M})$

$\mathrm{EtOH}(a q, 8.5 \mathrm{M})$

$\mathrm{H}_{2} \mathrm{O} / \mathrm{EtOH}(l, 1: 1)$

$\mathrm{H}_{2} \mathrm{O} / \mathrm{EtOH}(l, 1: 1)$

$\mathrm{H}_{2} \mathrm{O} / \mathrm{EtOH}(l, 1: 1)$

$\mathrm{H}_{2} \mathrm{O} / \mathrm{EtOH}(l, 83: 17)$

$\mathrm{H}_{2} \mathrm{O} / \mathrm{EtOH}(l, 1: 1)$

$\mathrm{H}_{2} \mathrm{O} / \mathrm{EtOH}(l, 4: 1)$

$\mathrm{H}_{2} \mathrm{O} / \mathrm{EtOH}(l, 4: 1)$

$\mathrm{H}_{2} \mathrm{O} / \mathrm{EtOH}(l, 1: 1)$

$\mathrm{EtOH}(l)$

$\mathrm{EtOH}(l)$

$\mathrm{H}_{2} \mathrm{O} / \mathrm{EtOH}(l, 97: 3)$

$\mathrm{H}_{2} \mathrm{O} / \mathrm{EtOH}(l, 97: 3)$

$\mathrm{H}_{2} \mathrm{O} / \mathrm{EtOH}(l, 9: 1)$

\section{$\mathrm{Hg}$}

$\mathrm{Hg}$

Xe

$\mathrm{Xe}$

$\mathrm{Hg}$

$\mathrm{Hg}$

$\mathrm{Hg}$

$\mathrm{Hg}$

$\mathrm{Hg}$

$\mathrm{Hg}$

W

$\mathrm{Hg}$

$\mathrm{Xe}$

$\mathrm{Xe}$

Xe

halogen $(>420 \mathrm{~nm})$

halogen $(>420 \mathrm{~nm})$

$\mathrm{Xe}(>365 \mathrm{~nm})$

$\mathrm{Hg}$

solar simulator

solar simulator

lar simulator

$\mathrm{Xe}$ (> $300 \mathrm{~nm}$ )

$\mathrm{Xe}(>300 \mathrm{~nm})$

$\mathrm{Xe}(>300 \mathrm{~nm})$

$\mathrm{Hg}$

solar simulator

solar

$\mathrm{Hg}$

$\mathrm{Hg}$

$\mathrm{Hg}$

UV

$\mathrm{Xe}-\mathrm{Hg}$

$\mathrm{Xe}-\mathrm{Hg}$

$\mathrm{Hg}$
150

(11.7)

500

200

125

125

25

200

00

350

350

300

(280)

(280)

300

450

150

150

150

150

150

150

150

500

500

500

500

$16 \times 176$

450

$\begin{array}{rrrrrrrrl}470 & - & - & - & - & - & - & 38 & {[279]} \\ 1627 & - & - & - & - & - & - & 50 & {[279]} \\ 427 & - & - & - & - & - & - & - & {[279]} \\ 2 & 5 & - & 3 & - & - & 0.1 & - & {[379]} \\ 361 & - & - & - & - & - & - & - & {[165]} \\ 794 & - & - & - & - & - & - & - & {[166]} \\ 805 & - & - & - & - & - & - & - & {[395]} \\ 104 & - & - & 23 & 13 & - & - & - & {[283]} \\ 13556 & - & - & 1140 & 800 & - & - & 2.6 & {[283]} \\ 461 & - & - & - & 39 & - & - & - & {[283]} \\ 160 & - & - & - & - & - & - & - & {[376]} \\ 540 & - & - & - & - & - & - & - & {[376]} \\ 18400 & - & - & - & - & - & - & - & {[376]} \\ 30200 & - & - & - & - & - & - & - & {[376]} \\ 227 & - & - & - & - & - & - & - & {[396]} \\ 251 & - & - & - & - & - & - & - & {[397]} \\ 2066 & - & - & - & - & - & - & 9.7 \\ 371 & - & - & - & - & - & - & - & {[217]} \\ 273 & - & - & - & - & - & - & - & {[233]} \\ 630 & - & - & - & - & - & - & - & {[158 \mathrm{a}]} \\ 164 & - & - & - & - & - & - & - & {[193]} \\ 64 & - & - & - & - & - & - & - & {[193]} \\ 11 & - & - & - & - & - & - & - & {[187]} \\ 1277 & - & - & - & - & - & - & - & {[172]} \\ 7541 & - & - & - & - & - & - & - & {[196 \mathrm{~b}]} \\ 9050 & - & - & - & - & - & - & - & {[196 \mathrm{~b}]} \\ 16968 & - & - & - & - & - & - & - & {[196 \mathrm{~b}]} \\ 10000 & - & - & - & - & - & - & - & {[196 \mathrm{a}]} \\ 100000 & - & - & - & - & - & - & - & {[196 \mathrm{a}]} \\ 60000 & - & - & - & - & - & - & - & {[196 \mathrm{a}]} \\ 213000 & 4096 & 7373 & 2458 & - & - & - & - & {[197]} \\ 6500 & 4149 & 7883 & 2351 & - & - & - & - & {[197]} \\ 8508 & - & - & - & - & - & - & - & {[195]} \\ 28571 & - & - & - & - & - & - & - & {[198]} \\ 95 & - & - & - & - & - & - & - & {[200]} \\ 3800 & - & - & - & - & - & - & - & {[203]} \\ 9600 & - & - & - & - & - & - & 13.3 & {[203]} \\ 215 & - & - & - & - & - & - & - & {[201 \mathrm{a}]} \\ 42 & - & - & 18 & - & - & - & - & {[201 \mathrm{c}]} \\ 240 & - & - & 30 & - & - & - & - & {[201 \mathrm{c}]} \\ 732 & - & - & - & - & - & - & - & {[201 \mathrm{~b}]} \\ 575 & - & - & - & - & - & - & - & {[201 \mathrm{~b}]} \\ 5500 & - & - & - & - & - & - & - & {[208]}\end{array}$


$\mathrm{Pt}(1 \%) / \mathrm{BaTi}_{4} \mathrm{O}_{9}$

$\mathrm{CoO}_{x}(1 \%) / \mathrm{BaTi}_{4} \mathrm{O}_{9}$

$\mathrm{CuO}_{x}(1 \%) / \mathrm{BaTi}_{4} \mathrm{O}$

$\mathrm{NiO}_{x}(1 \%) / \mathrm{BaTi}_{4} \mathrm{O}$

$\mathrm{Pt}(0.15 \%) / \mathrm{Y}_{2} \mathrm{Ta}_{2} \mathrm{O}_{5} \mathrm{~N}_{2}$

$\mathrm{Ru}(0.35 \%) / \mathrm{Y}_{2} \mathrm{Ta}_{2} \mathrm{O}_{5} \mathrm{~N}_{2}$

$\mathrm{Pt}(0.15 \%)-\mathrm{Ru}(0.35 \%) / \mathrm{Y}_{2} \mathrm{Ta}_{2} \mathrm{O}_{5} \mathrm{~N}_{2}$

$\mathrm{Pt}+\mathrm{H}_{3} \mathrm{PW}_{12} \mathrm{O}_{40}$

$\mathrm{Pt}(5 \%) / \mathrm{CdS}$

$\mathrm{Pt}(5 \%) / \mathrm{SiC}$

$\mathrm{Pt}(5 \%) / \mathrm{SiC}$

$\mathrm{Pt}(5 \%) / \mathrm{GaP}$

$\mathrm{Pt}(5 \%) / \mathrm{S}$

$\mathrm{Pt}(5 \%) / \mathrm{CdSe}$

$\mathrm{Pt}(5 \%) / \mathrm{MoSe}_{2}$

$\mathrm{Pt}(5 \%) / \mathrm{MoS}_{2}$

$\mathrm{Pt}(5 \%) / \mathrm{Fe}_{3} \mathrm{O}_{4}$

$\mathrm{Pt}(5 \%) / \mathrm{Fe}_{2} \mathrm{O}_{3}$

$\mathrm{Pt}(5 \%) / \mathrm{WO}_{3}$

$\mathrm{Pt}(5 \%) / \mathrm{WSe}_{2}$

$\mathrm{Pt}(5 \%) / \mathrm{GaAs}$

$\mathrm{Pt}(5 \%) / \mathrm{InP}$

$\mathrm{ZnS}$

$\mathrm{ZnS}$

$\mathrm{CdS} / Y^{[c]}$

$\mathrm{Pt}(0.5 \%) / \mathrm{CdS}$

$\mathrm{Pt}(1 \%) / \mathrm{Cd}_{0.6} \mathrm{Zn}_{0.4} \mathrm{~S} / \mathrm{Zn}(\mathrm{OH})_{2}(63 \%)$

$\operatorname{Pt}(15 \%)+\mathrm{CdS}$

$\mathrm{Pt}(15 \%) / \mathrm{CdS}_{0.75} \mathrm{Se}_{0.25}$

$\mathrm{MoS}_{2}(0.2 \%) / \mathrm{CdS}$

$\mathrm{Pt}(0.2 \%) / \mathrm{CdS}$

$\mathrm{CdS}(\mathrm{Si} / \mathrm{Cd}=50) / \mathrm{MCM}-48$

$\mathrm{CdS}(\mathrm{Si} / \mathrm{Cd}=40) / \mathrm{Ti}-\mathrm{MCM}-48$

$\mathrm{NiO}(3 \%) / \mathrm{CdS} / \mathrm{TiO}_{2}$ (nanorods)

$\mathrm{Ru}(5 \%) / \mathrm{ZnS}(\mathrm{Cd} / \mathrm{Zn}=4) / \mathrm{CdS}$

$\mathrm{Pt}(5 \%)+\mathrm{TiSe}_{2}$

$\operatorname{Pt}(0.1 \%) / \operatorname{Ag}(1.7 \%)-\operatorname{Ppy}(0.5 \%) / n-\mathrm{Si}^{[g]}$

$\mathrm{n}-\mathrm{Si}$

$\mathrm{Pt}(0.5 \%) / g-\mathrm{C}_{3} \mathrm{~N}_{4}$
$\mathrm{H}_{2} \mathrm{O} / \mathrm{EtOH}(l, 5: 1)$

$\mathrm{H}_{2} \mathrm{O} / \mathrm{EtOH}(l, 5: 1)$

$\mathrm{H}_{2} \mathrm{O} / \mathrm{EtOH}(l, 5: 1)$

$\mathrm{H}_{2} \mathrm{O} / \mathrm{EtOH}(l, 5: 1)$

$\mathrm{H}_{2} \mathrm{O} / \mathrm{EtOH}(l, 4: 1)$

$\mathrm{H}_{2} \mathrm{O} / \mathrm{EtOH}(l, 4: 1)$

$\mathrm{H}_{2} \mathrm{O} / \mathrm{EtOH}(l, 4: 1)$

$\mathrm{EtOH}(a q, 3.4 \mathrm{M})$

$\operatorname{EtOH}(a q, 0.17 \% \mathrm{w} / \mathrm{v})$

$\mathrm{H}_{2} \mathrm{O} / \mathrm{EtOH}(l, 1: 1)$

$\mathrm{H}_{2} \mathrm{O} / \mathrm{EtOH}(l, 1: 1)$

$\mathrm{H}_{2} \mathrm{O} / \mathrm{EtOH}(l, 1: 1)$

$\mathrm{H}_{2} \mathrm{O} / \mathrm{EtOH}(l, 1: 1)$

$\mathrm{H}_{2} \mathrm{O} / \mathrm{EtOH}(l, 1: 1)$

$\mathrm{H}_{2} \mathrm{O} / \mathrm{EtOH}(l, 1: 1)$

$\mathrm{H}_{2} \mathrm{O} / \mathrm{EtOH}(l, 1: 1)$

$\mathrm{H}_{2} \mathrm{O} / \mathrm{EtOH}(l, 1: 1)$

$\mathrm{H}_{2} \mathrm{O} / \mathrm{EtOH}(l, 1: 1)$

$\mathrm{H}_{2} \mathrm{O} / \mathrm{EtOH}(l, 1: 1)$

$\mathrm{H}_{2} \mathrm{O} / \mathrm{EtOH}(l, 1: 1)$

$\mathrm{H}_{2} \mathrm{O} / \mathrm{EtOH}(l, 1: 1)$

$\mathrm{H}_{2} \mathrm{O} / \mathrm{EtOH}(l, 5: 1)$

$\mathrm{H}_{2} \mathrm{O} / \mathrm{EtOH}(l, 7: 1)$

$\mathrm{H}_{2} \mathrm{O} / \mathrm{EtOH}(l, 1: 1)$

$\mathrm{H}_{2} \mathrm{O} / \mathrm{EtOH}(l, 9: 1)^{[f]}$

$\mathrm{H}_{2} \mathrm{O} / \mathrm{EtOH}(l, 1: 1)$

$\mathrm{H}_{2} \mathrm{O} / \mathrm{EtOH}(l, 1: 1)$

$\mathrm{H}_{2} \mathrm{O} / \mathrm{EtOH}(l, 9: 1)$

$\mathrm{H}_{2} \mathrm{O} / \mathrm{EtOH}(l, 9: 1)$

$\mathrm{H}_{2} \mathrm{O} / \mathrm{EtOH}(l, 1: 1)$

$\mathrm{H}_{2} \mathrm{O} / \mathrm{EtOH}(l, 1: 1)$

$\mathrm{H}_{2} \mathrm{O} / \mathrm{EtOH}(l, 4: 1)$

$\mathrm{H}_{2} \mathrm{O} / \mathrm{EtOH}(l, 9: 1)$

$\mathrm{EtOH}(l)$

$\mathrm{H}_{2} \mathrm{O} / \mathrm{EtOH}(l, 1: 1)$

$\mathrm{H}_{2} \mathrm{O} / \mathrm{EtOH}(l, 1: 1)$

$\mathrm{H}_{2} \mathrm{O} / \mathrm{EtOH}(l, 9: 1)$

$\mathrm{Hg}$

$\mathrm{Hg}$

$\mathrm{Hg}$

$\mathrm{Hg}$

$\mathrm{Xe}(>420 \mathrm{~nm})$

$\mathrm{Xe}(>420 \mathrm{~nm})$

$\mathrm{Xe}(>420 \mathrm{~nm})$

$\mathrm{Hg}$ (> $300 \mathrm{~nm})$

$\mathrm{Xe}$

$\mathrm{Xe}(>430 \mathrm{~nm})$

$\mathrm{Xe}$

$\mathrm{Xe}$

Xe

$\mathrm{Xe}$

Xe

$\mathrm{Xe}$

$\mathrm{Xe}$

Xe

$\mathrm{Xe}$

Xe

$\mathrm{Xe}$

$\mathrm{Xe}$

$\mathrm{Hg}(>290 \mathrm{~nm})$

$\mathrm{Hg}-\mathrm{Xe}$

$\mathrm{Xe}(>450 \mathrm{~nm})$

LED $(450 \mathrm{~nm})$

$\mathrm{Xe}(>320 \mathrm{~nm})$

$\mathrm{Xe}(>320 \mathrm{~nm})$

$\mathrm{Xe}(>420 \mathrm{~nm})$

$\mathrm{Xe}(>420 \mathrm{~nm})$

$\mathrm{Xe}(>400 \mathrm{~nm})$

$\mathrm{Xe}(>400 \mathrm{~nm})$

$\mathrm{Xe}(>420 \mathrm{~nm})$

$\mathrm{Xe}(>420 \mathrm{~nm})$

$\mathrm{Hg}(>320 \mathrm{~nm})$

W-halogen

W-halogen

$\mathrm{Xe}$ (> $420 \mathrm{~nm}$ )
$\mathrm{H}_{2} \mathrm{O} / \mathrm{EtOH}(l, 1: 1)$

$\mathrm{EtOH}(a q, 1 \mathrm{M}, \mathrm{pH} 3)$

500

500

500

500

300

500
500

500

500

500

500

500

500

500

500

500

500

500

150

125

500

750

(40)

500

500

300

300

300

300

300

(70)

400

300

300

300

${ }^{[f]} \mathrm{NaOH}(0.1 \mathrm{M}) .{ }^{[g]}$ Ppy: polypyrrole.
8550

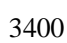

1400

500

170

833

2767

40

22
14

14
23
57

57

127

120
31

31
10

3
160

16

2500

33

173

2256

1

230

150
45

2730

34

2800

10
25

25 \begin{tabular}{ll}
11.7 & {$[204]$} \\
- & {$[204]$} \\
- & {$[204]$} \\
- & {$[204]$} \\
- & {$[209]$} \\
- & {$[209]$} \\
- & {$[209]$} \\
- & {$[398]$} \\
- & {$[214]$} \\
- & {$[214]$} \\
- & {$[214]$} \\
- & {$[214]$} \\
- & {$[214]$} \\
- & {$[214]$} \\
- & {$[214]$} \\
- & {$[214]$} \\
- & {$[214]$} \\
- & {$[214]$} \\
- & {$[214]$} \\
- & {$[214]$} \\
- & {$[214]$} \\
- & {$[214]$} \\
- & {$[399]$} \\
- & {$[231 b]$} \\
- & {$[219]$} \\
- & {$[213 a]$} \\
- & {$[230]$} \\
- & {$[235]$} \\
- & {$[235]$} \\
- & {$[292]$} \\
- & {$[292]$} \\
16.6 & {$[225 b]$} \\
36.3 & {$[225 a]$} \\
- & {$[156]$} \\
- & {$[224]$} \\
- & {$[349 b]$} \\
- & {$[100 \mathrm{e}]$} \\
- & {$[100 \mathrm{e}]$} \\
- & {$[247]$} \\
\hline &
\end{tabular}

$5)$. 
Table 4. Selected Photoreforming Data for Polyhydroxylated Substrates (Polyols)

\begin{tabular}{|c|c|c|c|c|c|c|c|c|c|c|c|c|}
\hline \multirow[b]{2}{*}{ photocatalyst } & \multirow[b]{2}{*}{ reaction medium } & \multirow[b]{2}{*}{ light source } & \multirow[b]{2}{*}{$\left(I / \mathrm{mW} \mathrm{cm}^{-2}\right)$} & \multirow[b]{2}{*}{$T /{ }^{\circ} \mathrm{C}$} & & & & & & & \multirow[b]{2}{*}{$\Phi_{\mathrm{a}} / \%$} & \multirow[b]{2}{*}{ reference } \\
\hline & & & & & $\mathrm{H}_{2}$ & $\mathrm{CO}_{2}$ & $\mathrm{CH}_{3} \mathrm{CHO}$ & $\mathrm{CH}_{4}$ & $\mathrm{CO}$ & others & & \\
\hline $\mathrm{Pt}(0.5 \%) /{ }^{\mathrm{P} 25} \mathrm{TiO}_{2}$ & glycerol $(a q, 0.368 \mathrm{mM})$ & solar simulator & 450 & 40 & 330 & 113 & - & - & - & - & - & [9] \\
\hline $\mathrm{Pt}(0.5 \%) /{ }^{\mathrm{P} 25} \mathrm{TiO}_{2}$ & $\operatorname{glycerol}(a q, 1.1 \mathrm{M})$ & solar simulator & 450 & 40 & 2813 & - & - & - & - & - & - & [9] \\
\hline $\mathrm{Pt}(0.5 \%) /{ }^{\mathrm{P} 25} \mathrm{TiO}_{2}$ & $\operatorname{glycerol}(a q, 17.7 \mathrm{mM})$ & solar simulator & 450 & 40 & 1151 & 300 & - & - & - & - & - & [24] \\
\hline $\mathrm{Pt}(0.5 \%) /{ }^{\mathrm{P} 25} \mathrm{TiO}_{2}$ & $\operatorname{glycerol}(a q, 20 \mathrm{mM})$ & solar simulator & 450 & - & 1950 & 525 & 205 & - & - & {$[a]$} & - & [345] \\
\hline $\mathrm{Pt}(2 \%) / \mathrm{TiO}_{2}$ & $\operatorname{glycerol}(a q, 0.61 \mathrm{~g} / \mathrm{L})$ & $\mathrm{Hg}$ & 100 & 20 & 5087 & 1387 & - & - & - & - & - & {$[39 \mathrm{~b}]$} \\
\hline $\mathrm{Pt}(0.1 \%) / \mathrm{TiO}_{2}(74 \%$ rutile $)$ & $\mathrm{H}_{2} \mathrm{O} /$ glycerol $(l, 4: 1)$ & $\mathrm{Xe}$ & 300 & - & 7784 & - & - & - & - & - & - & [94b] \\
\hline $\mathrm{Pt}(1 \%) / \mathrm{TiO}_{2}$ & $\operatorname{glycerol}(a q, 2.7 \mathrm{mM})$ & $\mathrm{Hg}$ & 125 & 30 & 6000 & 2500 & - & 18 & - & $4^{[b]}$ & - & [96] \\
\hline $\mathrm{Pt}(4 \%) / \mathrm{TiO}_{2}$ & $\mathrm{H}_{2} \mathrm{O} /$ glycerol $(1: 1)$ & $\mathrm{Xe}(>320 \mathrm{~nm})$ & 500 & - & 2500 & - & - & - & - & - & - & {$[366]$} \\
\hline $\mathrm{Pt}(0.5 \%) /{ }^{\mathrm{P} 25} \mathrm{TiO}_{2}+\mathrm{H}_{3} \mathrm{PW}_{12} \mathrm{O}_{40}(0.62 \mathrm{mM})$ & $\mathrm{H}_{2} \mathrm{O} / \operatorname{glycerol}(l, 9: 1, \mathrm{pH} 1.0)$ & halogen $(>420 \mathrm{~nm})$ & 300 & - & 143 & - & - & - & - & - & - & [400] \\
\hline $\mathrm{Pt}(0.5 \%))^{\mathrm{P} 25} \mathrm{TiO} 2+\mathrm{H}_{10} \mathrm{P}_{2} \mathrm{~W}_{17} \mathrm{O}_{61}(66 \mu \mathrm{M})$ & $\mathrm{H}_{2} \mathrm{O} /$ glycerol $(l, 9: 1)$ & halogen $(>420 \mathrm{~nm})$ & 300 & - & 131 & - & - & - & - & - & - & [401] \\
\hline $\mathrm{Pt}(0.5 \%){ }^{\mathrm{P} 25} \mathrm{TiO}_{2}+\mathrm{K}_{5} \mathrm{BW}_{11} \mathrm{O}_{39} \mathrm{H}_{4}(1 \mathrm{mM})$ & $\mathrm{H}_{2} \mathrm{O} /$ glycerol $(l, 9: 1)$ & halogen $(>420 \mathrm{~nm})$ & 300 & - & 130 & - & - & - & - & - & - & [402] \\
\hline $\mathrm{Pt}(0.3 \%) /{ }^{\mathrm{P} 25} \mathrm{TiO}_{2}$ & $\operatorname{glycerol}(a q, 0.65 \mathrm{mM}, \mathrm{pH} 12)$ & UV (366 nm) & $2 \times 15$ & - & 317 & - & - & - & - & - & 1.1 & [325] \\
\hline $\mathrm{Pt}(2 \%) /{ }^{\mathrm{P} 25} \mathrm{TiO}_{2}$ & $\operatorname{glycerol}(a q, 8.3 \mathrm{mM})$ & $\mathrm{Hg}$ & 100 & 20 & 4285 & 1836 & - & - & - & - & - & [39a] \\
\hline $\mathrm{Pt}(1 \%) / /^{\mathrm{P} 25} \mathrm{TiO}_{2}$ & $\mathrm{H}_{2} \mathrm{O} / \operatorname{glycerol}(l, 30: 1)$ & $\mathrm{Xe}(>320 \mathrm{~nm})$ & 300 & 10 & 4280 & - & - & - & - & - & - & [334] \\
\hline $\mathrm{Pt}(2.1 \%) / \mathrm{TiO}_{2}$ & $\operatorname{glycerol}(a q, 7.34 \mathrm{M})$ & UV $(300-400 \mathrm{~nm})$ & $12 \times 15$ & - & 6335 & 72 & 6 & - & - & - & - & [330] \\
\hline $\mathrm{CuO}(0.4 \%) /{ }^{\mathrm{P} 25} \mathrm{TiO}_{2}$ & $\operatorname{glycerol}(a q, 1 \mathrm{M})$ & $\mathrm{Xe}$ & 250 & - & 863 & 90 & - & - & - & - & - & {$[272 b]$} \\
\hline $\mathrm{Pt}(0.5 \%) / \mathrm{TiO}_{2}$ (porous) & $\mathrm{H}_{2} \mathrm{O} /$ glycerol $(l, 4: 1)$ & $\mathrm{Hg}$ & 500 & 30 & 6000 & - & - & - & - & - & - & [120a] \\
\hline $\mathrm{Pt}(2 \%) / \mathrm{F}: \mathrm{TiO}_{2}$ (nanosheets) & $\operatorname{glycerol}(a q, 0.1 \mathrm{M})$ & $\mathrm{Xe}$ & (20) & - & 8455 & - & - & - & - & - & - & {$[121 \mathrm{a}]$} \\
\hline $\mathrm{Pt} /(\mathrm{B}, \mathrm{N}): \mathrm{TiO}_{2}$ & $\mathrm{H}_{2} \mathrm{O} /$ glycerol $(l, 19: 1)$ & $\mathrm{Xe}$ & 300 & - & 8133 & - & - & - & - & - & - & [359] \\
\hline $\mathrm{Pt} / \mathrm{N}: \mathrm{TiO}_{2}$ & $\mathrm{H}_{2} \mathrm{O} / \operatorname{glycerol}(l, 19: 1)$ & $\mathrm{Xe}$ & 300 & - & 5133 & - & - & - & - & - & - & [359] \\
\hline $\mathrm{Pt} / \mathrm{B}: \mathrm{TiO}_{2}$ & $\mathrm{H}_{2} \mathrm{O} / \operatorname{glycerol}(l, 19: 1)$ & $\mathrm{Xe}$ & 300 & - & 4333 & - & - & - & - & - & - & [359] \\
\hline $\mathrm{P}(5 \%): \mathrm{TiO}_{2}$ & $\mathrm{H}_{2} \mathrm{O} / \operatorname{glycerol}(l, 19: 1)$ & $\mathrm{Hg}$ & 500 & 60 & 1400 & - & - & - & - & - & - & {$[175]$} \\
\hline $\mathrm{Pt}(0.3 \%) / \mathrm{Gd}(0.5 \%): \mathrm{TiO}_{2}$ & $\mathrm{H}_{2} \mathrm{O} / \operatorname{glycerol}(l, 50: 1)$ & $\mathrm{Hg}$ & 300 & - & 9900 & - & - & - & - & - & - & [373] \\
\hline $\mathrm{Pd}(0.5 \%) /{ }^{\mathrm{P} 25} \mathrm{TiO}_{2}$ & $\mathrm{H}_{2} \mathrm{O} /$ glycerol $(1,999: 1)$ & $\mathrm{Xe}$ & 400 & - & 895 & - & - & - & - & - & - & [90a] \\
\hline $\mathrm{Pd}(0.5 \%) / /^{\mathrm{P} 25} \mathrm{TiO}_{2}$ & $\mathrm{H}_{2} \mathrm{O} /$ glycerol $(1,999: 1)$ & $\mathrm{Xe}$ & 400 & - & 1075 & - & - & - & - & - & - & [346] \\
\hline $\mathrm{Au}(2 \%) /{ }^{\mathrm{P} 25} \mathrm{TiO}_{2}$ & $\mathrm{H}_{2} \mathrm{O} /$ glycerol $(1,999: 1)$ & $\mathrm{Xe}$ & 400 & - & 590 & - & - & - & - & - & - & [346] \\
\hline${ }^{\mathrm{P} 25} \mathrm{TiO}_{2}$ & $\mathrm{H}_{2} \mathrm{O} /$ glycerol $(l, 9: 1)$ & UV $(365 \mathrm{~nm})$ & $(6.5)$ & - & 1900 & - & - & - & - & - & - & [115] \\
\hline $\mathrm{Au}(0.5 \%) / /^{\mathrm{P} 25} \mathrm{TiO}_{2}$ & $\mathrm{H}_{2} \mathrm{O} /$ glycerol $(l, 9: 1)$ & UV $(365 \mathrm{~nm})$ & (6.5) & - & 27900 & - & - & - & - & - & - & [115] \\
\hline $\mathrm{Au}(0.5 \%) / \mathrm{TiO}_{2}$ (anatase) & $\mathrm{H}_{2} \mathrm{O} /$ glycerol $(l, 9: 1)$ & UV $(365 \mathrm{~nm})$ & (6.5) & - & 15000 & - & - & - & - & - & - & [115] \\
\hline $\mathrm{Au}(0.5 \%) / \mathrm{TiO}_{2}$ (brookite) & $\mathrm{H}_{2} \mathrm{O} /$ glycerol $(l, 9: 1)$ & UV $(365 \mathrm{~nm})$ & $(6.5)$ & - & 13800 & - & - & - & - & - & - & [115] \\
\hline $\mathrm{Au}(0.5 \%) / \mathrm{TiO}_{2}($ rutile $)$ & $\mathrm{H}_{2} \mathrm{O} /$ glycerol $(l, 9: 1)$ & UV $(365 \mathrm{~nm})$ & $(6.5)$ & - & 3200 & - & - & - & - & - & - & [115] \\
\hline $\mathrm{Au}(0.5 \%) / \mathrm{TiO}_{2}$ (anatase nanotubes) & $\mathrm{H}_{2} \mathrm{O} / \operatorname{glycerol}(l, 9: 1)$ & UV $(365 \mathrm{~nm})$ & $(6.5)$ & - & 29200 & - & - & - & - & - & - & [115] \\
\hline $\mathrm{Au}(1 \%) / \mathrm{TiO}_{2}($ anatase $)$ & $\mathrm{H}_{2} \mathrm{O} /$ glycerol $(l, 1: 1)$ & $\mathrm{Xe}$ & 300 & 25 & 4900 & - & - & - & - & - & - & [326] \\
\hline $\mathrm{Pd}(1 \%) /{ }^{\mathrm{P} 25} \mathrm{TiO}_{2}$ & $\mathrm{H}_{2} \mathrm{O} /$ glycerol $(l, 3: 1)$ & UV LED (365 nm) & (60) & - & 9846 & - & - & - & - & - & - & [287] \\
\hline $\mathrm{Au}_{3} \mathrm{Pd}(1 \%) /{ }^{\mathrm{P} 25} \mathrm{TiO}_{2}$ & $\mathrm{H}_{2} \mathrm{O} /$ glycerol $(l, 3: 1)$ & UV LED $(365 \mathrm{~nm})$ & (60) & - & 13231 & - & - & - & - & - & - & [287] \\
\hline $\mathrm{Pd}_{3} \mathrm{Au}(1 \%) / /^{\mathrm{P} 25} \mathrm{TiO}_{2}$ & $\mathrm{H}_{2} \mathrm{O} /$ glycerol $(l, 3: 1)$ & UV LED $(365 \mathrm{~nm})$ & (60) & - & 14769 & - & - & - & - & - & - & [287] \\
\hline $\left.\mathrm{Au}_{\text {shell }} \mathrm{Pd}_{\text {core }}(1 \%)\right)^{\mathrm{P25}} \mathrm{TiO}_{2}$ & $\mathrm{H}_{2} \mathrm{O} /$ glycerol $(l, 3: 1)$ & UV LED $(365 \mathrm{~nm})$ & (60) & - & 14923 & - & - & - & - & - & - & [287] \\
\hline $\left.\mathrm{Pd}_{\text {shell }} \mathrm{Au}_{\text {core }}(1 \%)\right)^{/ 25} \mathrm{TiO}_{2}$ & $\mathrm{H}_{2} \mathrm{O} /$ glycerol $(l, 3: 1)$ & UV LED (365 nm) & $(60)$ & - & 19631 & - & - & - & - & - & - & [287] \\
\hline$\left.\mathrm{Pd}_{\text {shell }} \mathrm{Au}_{\text {core }}(1 \%)\right|^{\mathrm{P25}} \mathrm{TiO}_{2}$ & $\mathrm{H}_{2} \mathrm{O} /$ crude glycerol $(l, 3: 1)$ & UV LED $(365 \mathrm{~nm})$ & $(60)$ & - & 10109 & - & - & - & - & - & - & [287] \\
\hline $\mathrm{Au}(1 \%) / \mathrm{TiO}_{2} /$ cordierite honeycomb & $\mathrm{H}_{2} \mathrm{O} /$ glycerol $(l, 1: 1 \mathrm{~mol})$ & UV fiber optics (365 nm) & $(0.2)$ & 25 & 768 & - & - & - & - & - & 32.0 & [392] \\
\hline
\end{tabular}


$\mathrm{Au}(1 \%) / \mathrm{TiO}_{2} /$ cordierite honeycomb $\mathrm{CuO}_{x}(2.5 \%) @ \mathrm{TiO}_{2}$

$\mathrm{CuO}_{x}(1 \%) / \mathrm{TiO}_{2}$

$\mathrm{CuO}_{x}(1 \%) / \mathrm{TiO}_{2}$

${ }^{\mathrm{P} 25} \mathrm{TiO}_{2}$

$\mathrm{CuO}(1.3 \%) /^{\mathrm{P} 25} \mathrm{TiO}_{2}$

$\mathrm{CuO}(1.25 \%) /{ }^{\mathrm{P2} 25} \mathrm{TiO}_{2}$

$\mathrm{Pt}(1.25 \%) /{ }^{\mathrm{P2} 5} \mathrm{TiO}_{2}$

$\mathrm{Cu}_{2} \mathrm{O}^{/ 25} \mathrm{TiO}_{2}$

$\mathrm{Cu}(10 \% \mathrm{~mol}) / \mathrm{TiO}_{2}$

$\mathrm{Cu}(1.5 \%) / \mathrm{TiO}_{2}$ (nanorods)

$\mathrm{TiO}_{2}$ (nanorods)

${ }^{\mathrm{P} 25} \mathrm{TiO}_{2}$

$\mathrm{CuO}(1.5 \%) / \mathrm{TiO}_{2}$ (nanotubes)

$\mathrm{NiO}(2 \%) / \mathrm{TiO}_{2}$ (anatase/rutile $=7: 3$ )

$\mathrm{CuO}(2 \%) / \mathrm{TiO}_{2}$ (anatase/rutile $=7: 3$ )

$\mathrm{CoO}(2 \%) / \mathrm{TiO}_{2}$ (anatase/rutile $\left.=7: 3\right)$

$\mathrm{N}: \mathrm{Cu}(2.5 \%$ vs. Ti $) /{ }^{225} \mathrm{TiO}_{2}$

$\mathrm{RGO}(3 \%) / \mathrm{TiO}_{2}$

$\mathrm{Cu}_{2} \mathrm{O}(1 \%) / \mathrm{TiO}_{2}$

$\mathrm{TiO}_{2}$

$\mathrm{NiO}(10 \%) / \mathrm{TiO}_{2}$

$\mathrm{NiO}$

$\mathrm{Sn}(0.24 \%) / \mathrm{RuO}_{2}(0.68 \%) /{ }^{\mathrm{P} 25} \mathrm{TiO}_{2}$

$\mathrm{RGO}(3 \%)-\mathrm{Cu}_{2} \mathrm{O}(1 \%) / \mathrm{TiO}_{2}$

$\mathrm{ZnO}$ (nanorods)/RGO(12\%)

$\mathrm{ZnO} / \mathrm{RGO}$

$\mathrm{ZnO} @ \mathrm{Bi}_{2} \mathrm{~S}_{3} / \mathrm{ZnS} / \mathrm{RGO}$

$\mathrm{Pt}(0.5 \%) / \mathrm{Au}(3 \%) / \mathrm{WO}_{3}$

$\mathrm{Bi}_{2} \mathrm{WO}_{6}$

$\beta-\mathrm{Fe}_{2} \mathrm{O}_{3} / \mathrm{Si}(100) \mathrm{CVD}$

$\varepsilon-\mathrm{Fe}_{2} \mathrm{O}_{3} / \mathrm{Si}(100) \mathrm{CVD}$

$\mathrm{ZnS} / \mathrm{ZnO}$ (nanotube arrays)

$\mathrm{ZnS}$

$\mathrm{ZnO}$ (nanotube arrays)

$\mathrm{ZnS}(\mathrm{ZnS} / \mathrm{ZnO}=1.5 \mathrm{~mol}) / \mathrm{ZnO}$ (nanorods)

$\mathrm{ZnS}(\mathrm{ZnS} / \mathrm{ZnO}=1.5 \mathrm{~mol}) / \mathrm{ZnO}$ (nanorods)

$\mathrm{MoS}_{2}(0.2 \%) / \mathrm{CdS}$

$\mathrm{Pt}(0.2 \%) / \mathrm{CdS}$

$\mathrm{Pt} / \mathrm{CdS}$

$\mathrm{Pt}(0.5 \%) / \mathrm{Cd}_{0.5} \mathrm{Zn}_{0.5} \mathrm{~S}$

$\mathrm{Pt} / \mathrm{Cd}_{0.6} \mathrm{Zn}_{0.4} \mathrm{~S}:\left(\gamma-\mathrm{Zn}(\mathrm{OH})_{2}\right)$

$\mathrm{Pt}(5 \%)+\mathrm{TiO}_{2}$ (anatase)
$\mathrm{H}_{2} \mathrm{O} /$ bio-glycerol $(l, 1: 1 \mathrm{~mol})$ $\operatorname{glycerol}(a q, 1 \mathrm{M})$

glycerol $(a q, 1 \mathrm{M})$

glycerol $(a q, 1 \mathrm{M})$

$\mathrm{H}_{2} \mathrm{O}$ /glycerol $(0.1 \mathrm{M})$

$\mathrm{H}_{2} \mathrm{O} /$ glycerol $(0.1 \mathrm{M})$

$\mathrm{H}_{2} \mathrm{O} /$ crude glycerol $(l, 1.04 \%)$

$\mathrm{H}_{2} \mathrm{O} /$ crude glycerol $(l, 5.2 \%)$

$\operatorname{glycerol}(a q, 0.1 \mathrm{M})$

$\mathrm{H}_{2} \mathrm{O} /$ glycerol $(l, 9: 1)$

$\mathrm{H}_{2} \mathrm{O} /$ glycerol $(l, 19: 1)$

$\mathrm{H}_{2} \mathrm{O} /$ glycerol $(l, 19: 1)$

$\mathrm{H}_{2} \mathrm{O} /$ glycerol $(l, 19: 1)$

$\mathrm{H}_{2} \mathrm{O} /$ glycerol $(l, 19: 1)$

$\mathrm{H}_{2} \mathrm{O} / \operatorname{glycerol}(l, 9: 1)$

$\mathrm{H}_{2} \mathrm{O} /$ glycerol $(l, 9: 1)$

$\mathrm{H}_{2} \mathrm{O} /$ glycerol $(l, 9: 1)$

$\mathrm{H}_{2} \mathrm{O} /$ glycerol $(l, 9: 1)$

$\mathrm{H}_{2} \mathrm{O} /$ glycerol $(l, 19: 1)$

$\mathrm{H}_{2} \mathrm{O} / \operatorname{glycerol}(l, 19: 1)$

$\mathrm{H}_{2} \mathrm{O} /$ glycerol $(l, 5: 1)$

$\mathrm{H}_{2} \mathrm{O} /$ glycerol $(l, 5: 1)$

$\mathrm{H}_{2} \mathrm{O} /$ glycerol $(l, 5: 1)$

$\mathrm{H}_{2} \mathrm{O} /$ glycerol $(l, 33: 1)$

$\mathrm{H}_{2} \mathrm{O} /$ glycerol $(l, 19: 1)$

$\mathrm{H}_{2} \mathrm{O} /$ glycerol $(l, 19: 1)$

$\mathrm{H}_{2} \mathrm{O} / \operatorname{glycerol}(l, 19: 1)$

$\mathrm{H}_{2} \mathrm{O} /$ glycerol $(l, 19: 1)$

glycerol $(a q, 2 \mathrm{mM})$

$\mathrm{H}_{2} \mathrm{O} /$ glycerol $(l, 1: 1)$

$\operatorname{glycerol}(l, 1.0 \mathrm{M})$

$\operatorname{glycerol}(l, 1.0 \mathrm{M})$

$\mathrm{H}_{2} \mathrm{O} /$ glycerol $(l, 9: 1)$

$\mathrm{H}_{2} \mathrm{O} /$ glycerol $(l, 9: 1)$

$\mathrm{H}_{2} \mathrm{O} / \operatorname{glycerol}(l, 9: 1)$

$\mathrm{H}_{2} \mathrm{O} /$ glycerol $(l, 93: 7)$

$\mathrm{H}_{2} \mathrm{O} / \operatorname{glycerol}(l, 93: 7)$

$\mathrm{H}_{2} \mathrm{O} / \operatorname{glycerol}(l, 9: 1)$

$\mathrm{H}_{2} \mathrm{O} /$ glycerol $(l, 9: 1)$

$\mathrm{H}_{2} \mathrm{O} / \operatorname{glycerol}(l, 9: 11)$

$\operatorname{glycerol}(a q, 1.368 \mathrm{M})^{[e]}$

$\operatorname{glycerol}(l, 1: 1)^{[f]}$

ethylene glycol(aq, $0.1 \mathrm{M})$
UV fiber optics (365 nm)

$\mathrm{Hg}$

solar simulator

UV LED (365 nm)

UV LED

UVA $(340 \mathrm{~nm})$

UVA $(340 \mathrm{~nm})$

$\mathrm{Xe}(>420 \mathrm{~nm})$

halogen

sunlight

sunlight

sunlight

sunlight

$\mathrm{Hg}$

$\mathrm{Hg}$

$\mathrm{Hg}$

$\mathrm{Hg}(>320 \mathrm{~nm})$

$\mathrm{Hg}$

$\mathrm{Hg}$

$\mathrm{Hg}$

$\mathrm{Hg}$

$\mathrm{Hg}$

$\mathrm{Hg}$

$\mathrm{Xe}$

$\mathrm{Xe}$

$\mathrm{Xe}(450-600 \mathrm{~nm})$

$\mathrm{Xe}($ solar, > $300 \mathrm{~nm})$

$\mathrm{Xe}$ (solar, > $300 \mathrm{~nm}$ )

$\mathrm{Xe}$

$\mathrm{Hg}$

$\mathrm{Xe}$ (solar simulator)

$\mathrm{Xe}(>420 \mathrm{~nm})$

$\mathrm{Xe}(>420 \mathrm{~nm})$

$\mathrm{Hg}-\mathrm{Xe}(>418 \mathrm{~nm})$

$\mathrm{Hg}(>420 \mathrm{~nm})$

$\mathrm{Hg}-\mathrm{Xe}(>418 \mathrm{~nm})$

$\mathrm{Hg}$

00
25

300

[272a]

- [277]

- [277]

- [274]

- [274]

- [276]

- [276]

- [403]

- [323]

- [128a]

- [128a]

- [128a]

- [336]

- [280]

- [280]

- [280]

- [167]

- [301]

- [301]

- [347]

- [347]

- [347]

5.8 [283]

- [301]

- [190]

- [232]

- [232]

- [186]

- [210]

- [196a]

- [196a]

- [191a]

- [191a]

- [191a]

- [191b]

- [191b]

- [292]

- [292]

- [223b]

- [64]

- [229]

$28^{[c]}-[59]$ 
$\mathrm{Pt}(4 \%) / \mathrm{TiO}_{2}$

$\mathrm{Pt}(2 \%) / \mathrm{TiO}_{2}$

$\mathrm{Pt}(2.1 \%) / \mathrm{TiO}_{2}$

$\mathrm{Pt}(0.6 \%) / \mathrm{TiO}_{2}$ (mesoporous)

$\mathrm{Pt}(0.3 \%) / \mathrm{Gd}: \mathrm{TiO}_{2}$

$\mathrm{Au}(2.26 \%) / \mathrm{TiO}_{2}$

$\mathrm{Au}(1.5 \%) /{ }^{\mathrm{P} 25} \mathrm{TiO}_{2}$

$\mathrm{Au}(1.5 \%) / \mathrm{TiO}_{2}$ (anatase)

$\mathrm{Au}(0.5 \%) / \mathrm{TiO}_{2}$ (anatase nanotubes)

$\mathrm{Au}(1.5 \%) /{ }^{\mathrm{P} 25} \mathrm{TiO}_{2}$

$\mathrm{Pt}(2 \%) /{ }^{\mathrm{P} 25} \mathrm{TiO}_{2}$

$\mathrm{Pt}(2 \%) / \mathrm{TiO}_{2}$

$\mathrm{Pt}(2 \%) / \mathrm{P}^{25} \mathrm{TiO}_{2}$

$\mathrm{H}_{2} \mathrm{O} /$ ethylene $\operatorname{glycol}(l, 1: 1)$

ethylene glycol $(l)$

$\mathrm{Xe}(>320 \mathrm{~nm})$

thylene $\operatorname{glycol}(a q, 7.34 \mathrm{M})$

$\mathrm{H}_{2} \mathrm{O}$ /ethylene $\operatorname{glycol}(l, 10: 1)$

$\mathrm{H}_{2} \mathrm{O} /$ ethylene glycol $(l, 50: 1)$

ethylene $\operatorname{glycol}(a q, 1 \mathrm{M})$

$\mathrm{H}_{2} \mathrm{O} /$ ethylene glycol $(l, 9: 1)$

$\mathrm{H}_{2} \mathrm{O}$ /ethylene glycol $(l, 9: 1)$

$\mathrm{H}_{2} \mathrm{O} /$ ethylene glycol $(l, 9: 1)$

$\mathrm{H}_{2} \mathrm{O}$ /ethylene glycol $(l, 9: 1)$

erythritol( $a q, 8.3 \mathrm{mM})$

$\operatorname{arabitol}(a q, 1.0 \mathrm{~g} / \mathrm{L})$

$\operatorname{arabitol}(a q, 8.3 \mathrm{mM})$

UV (300-400 nm)

$\mathrm{Hg}(>320 \mathrm{~nm})$

$\mathrm{Hg}$

UV $(365 \mathrm{~nm})$

UV (365 nm)

UV $(365 \mathrm{~nm})$

UV (365 nm)

$\mathrm{Hg}$

$\mathrm{Hg}$
$500-1933$

1933
8762

$\begin{array}{lll}(12 \times 15) & - & 7565 \\ 300 & - & 2255\end{array}$

$300-2255$

$500-40-14600$

20900

$\begin{array}{lll}(6.5) & - & 12000\end{array}$

(6.5) - $\quad 22700$

- $\quad 23100$

$100 \quad 20 \quad 4750 \quad 2106$

$\begin{array}{llll}100 & 20 & 2497 & 532\end{array}$

$\begin{array}{llll}100 & 20 & 5631 & 2571\end{array}$
- [366]

$[108 \mathrm{~d}]$

$0.6^{[d]} \quad-\quad[330]$

$-\quad-\quad[140 \mathrm{~b}]$

- $[373]$

- [268c]

$-\quad[115]$

- $\quad[115]$

$-[115]$

$-$

$-\quad[39 a]$

- $\quad[39 \mathrm{~b}]$

- $\quad[39 \mathrm{a}]$

${ }^{[a]}$ Acetol, methanol and ethanol formed at $28,13,40 \mu \mathrm{mol} \mathrm{gcat}^{-1} \mathrm{~h}^{-1}$, respectively. ${ }^{[b]}$ Ethane. ${ }^{[c]}$ Glycolaldehyde. ${ }^{[d]}$ Formic acid. ${ }^{[e]}$ Solution containing NaOH$(1 \mathrm{M}) .{ }^{[f]}$ Solution containing NaCl$(1.5 \mathrm{M})$.

Table 5. Selected Photoreforming Data for Aldehydes

\begin{tabular}{|c|c|c|c|c|c|c|c|c|}
\hline \multirow[b]{2}{*}{ photocatalyst } & \multirow[b]{2}{*}{ reaction medium } & \multirow[b]{2}{*}{ light source } & \multirow{2}{*}{$\begin{array}{l}P / \mathrm{W} \\
\left(I / \mathrm{mW} \mathrm{cm}^{-2}\right)\end{array}$} & \multirow[b]{2}{*}{$T /{ }^{\circ} \mathrm{C}$} & \multicolumn{2}{|c|}{ production rates $/ \mu \mathrm{mol} \mathrm{g} \mathrm{cat}^{-1} \mathrm{~h}^{-1}$} & \multirow[b]{2}{*}{$\Phi_{\mathrm{a}} / \%$} & \multirow[b]{2}{*}{ reference } \\
\hline & & & & & $\mathrm{H}_{2}$ & $\mathrm{CO}_{2}$ & & \\
\hline $\mathrm{Pt}(2 \%) / \mathrm{TiO}_{2}$ & formaldehyde $\left(a q, 0.20 \mathrm{~g} \mathrm{~L}^{-1}\right)$ & $\mathrm{Hg}$ & 100 & 20 & 939 & 384 & - & [39b] \\
\hline $\mathrm{Pd}(0.5 \%) / \mathrm{TiO}_{2}$ & formaldehyde(aq, 18.6 M) & $\mathrm{Xe}$ & 400 & - & 1700 & - & - & {$[57]$} \\
\hline $\mathrm{Pt}(0.5 \%))^{225} \mathrm{TiO}_{2}$ & formaldehyde $(a q, 0.01 \mathrm{M})$ & $\mathrm{Hg}$ & 250 & - & 550 & - & - & [81] \\
\hline $\mathrm{Au}(0.43 \%) /{ }^{p 25} \mathrm{TiO}_{2}$ & formaldehyde $(a q, 0.05 \mathrm{M})$ & $\mathrm{Xe}$ & 300 & - & 247 & - & - & [94a] \\
\hline $\mathrm{Cu}_{2} \mathrm{O}(110)$ & $\mathrm{H}_{2} \mathrm{O} /$ formaldehyde $(l, 4: 1)$ & $\mathrm{Xe}$ & (5) & r.t. & 2740 & - & - & [348] \\
\hline $\mathrm{ZnO}$ & $\mathrm{H}_{2} \mathrm{O} /$ formaldehyde $(l, 9: 1)$ & $\mathrm{Xe}$ & 300 & r.t. & 33750 & - & - & [189] \\
\hline $\mathrm{SrTiO}_{3}$ & formaldehyde(aq, $1 \mathrm{M})$ & $\mathrm{Hg}$ & 150 & - & 41 & - & - & {$[201 d]$} \\
\hline $\mathrm{Ni} / \mathrm{LaNiO}_{3-\mathrm{x}}-\mathrm{La}_{2} \mathrm{O}_{2} \mathrm{CO}_{3}$ & $\mathrm{H}_{2} \mathrm{O} /$ formaldehyde $(l, 8: 1)$ & $\mathrm{Xe}(>400 \mathrm{~nm})$ & 125 & 50 & 35 & - & - & [404] \\
\hline $\mathrm{Cu}(\mathrm{Fe} / \mathrm{Cu}=4) / \mathrm{LaFeO}_{3}$ & $\mathrm{H}_{2} \mathrm{O} /$ formaldehyde $(l, 7: 1)$ & $\mathrm{Xe}(>400 \mathrm{~nm})$ & 125 & - & 343 & - & - & {$[211]$} \\
\hline $\operatorname{Pt}(3.8 \%)+\mathrm{CdS}$ & formaldehyde( $a q, 5 \mathrm{M}, \mathrm{pH} 5)$ & $\mathrm{Hg}(>400 \mathrm{~nm})$ & 500 & - & 77 & {$[a]$} & - & [218] \\
\hline $\mathrm{Pt}(0.5 \%) / \mathrm{TiO}_{2}$ & acetaldehyde(aq, $0.89 \mathrm{mM})$ & solar simulator & 280 & 40 & 75 & - & - & {$[38 \mathrm{a}]$} \\
\hline $\mathrm{Pt}(0.5 \%) / \mathrm{TiO}_{2}$ (porous) & $\mathrm{H}_{2} \mathrm{O} /$ acetaldehyde $(l, 4: 1)$ & $\mathrm{Hg}$ & 500 & 30 & 3000 & - & - & [120a] \\
\hline $\mathrm{Pt}(2 \%) / \mathrm{TiO}_{2}$ & glyceraldehyde $\left(a q, 0.60 \mathrm{~g} \mathrm{~L}^{-1}\right)$ & $\mathrm{Hg}$ & 100 & 20 & 2241 & 790 & - & {$[39 b]$} \\
\hline $\mathrm{Pt}(4.85 \%) /{ }^{\mathrm{P} 25} \mathrm{TiO}_{2}$ & $\mathrm{H}_{2} \mathrm{O} / \operatorname{propanal}(l, 1: 1)$ & $\mathrm{Hg}$ & 125 & - & 19 & - & - & {$[43]$} \\
\hline
\end{tabular}

${ }^{[a]}$ Traces of methanol were observed. 
Table 6. Selected Photoreforming Data for Carboxylic Acids (or Carboxylates)

\begin{tabular}{|c|c|c|c|c|c|c|c|c|c|c|c|c|}
\hline \multirow[b]{2}{*}{ photocatalyst } & \multirow[b]{2}{*}{ reaction medium } & \multirow[b]{2}{*}{ light source } & \multirow{2}{*}{$\begin{array}{l}P / \mathrm{W} \\
\left(I / \mathrm{mW} \mathrm{cm}^{-2}\right)\end{array}$} & \multirow[b]{2}{*}{$T /{ }^{\circ} \mathrm{C}$} & \multicolumn{6}{|c|}{ production rates $/ \mu$ mol g $\mathrm{gat}^{-1} \mathrm{~h}^{-1}$} & \multirow[b]{2}{*}{$\Phi_{\mathrm{a}} / \%$} & \multirow[b]{2}{*}{ reference } \\
\hline & & & & & $\mathrm{H}_{2}$ & $\mathrm{CO}_{2}$ & $\mathrm{CH}_{4}$ & $\mathrm{CO}$ & $\mathrm{C}_{2} \mathrm{H}_{6}$ & others & & \\
\hline $\mathrm{Pt}(0.5 \%) / \mathrm{TiO}_{2}$ & formic $\operatorname{acid}(a q, 1.3 \mathrm{mM})$ & solar & 280 & 40 & 1275 & - & - & - & - & - & - & [38a] \\
\hline $\mathrm{Pt}(0.5 \%) /{ }^{\mathrm{P} 25} \mathrm{TiO}_{2}$ & formic $\operatorname{acid}(a q, 0.01 \mathrm{M})$ & $\mathrm{Hg}$ & 250 & - & 1150 & - & - & - & - & - & - & [81] \\
\hline $\mathrm{Pt}(2 \%) / \mathrm{TiO}_{2}$ (anatase) & formic $\operatorname{acid}(v, 6$ Torr $)$ & $\mathrm{Hg}$ & 500 & r.t. & 50.3 & 54.2 & - & - & - & - & - & {$[315]$} \\
\hline $\mathrm{Pt}(0.5 \%) / \mathrm{TiO}_{2}$ & $\mathrm{H}_{2} \mathrm{O} /$ formic $\operatorname{acid}(v, 10: 1)$ & $\mathrm{Hg}$ & 250 & 55 & 5400 & 4100 & - & 80 & - & - & - & {$[328]$} \\
\hline $\mathrm{Au}(0.43 \%) /{ }^{\mathrm{P} 25} \mathrm{TiO}_{2}$ & formic $\operatorname{acid}(a q, 0.05 \mathrm{M})$ & $\mathrm{Xe}$ & 300 & - & 452 & - & - & - & - & - & - & [94a] \\
\hline $\mathrm{Cu} / \mathrm{TiO}_{2}$ (anatase) & formic $\operatorname{acid}(a q, 1 \mathrm{M})^{[a]}$ & UV & 125 & 25 & 5000 & - & - & - & - & - & - & {$[320]$} \\
\hline (Ti,Cr):MCM-41 & $\mathrm{H}_{2} \mathrm{O} /$ formic $\operatorname{acid}(l, 3: 1)$ & $\mathrm{Xe}(>430 \mathrm{~nm})$ & 350 & - & 23.2 & - & - & - & - & - & - & [405] \\
\hline $\mathrm{SrTiO}_{3}$ & formic $\operatorname{acid}(a q, 1 \mathrm{M})$ & $\mathrm{Hg}$ & 150 & - & 280 & - & - & - & - & - & - & {$[201 d]$} \\
\hline $\mathrm{Pt}(0.5 \%) / \mathrm{Cu}_{2} \mathrm{O}$ & formic $\operatorname{acid}(a q, 2.5 \mathrm{M}, \mathrm{pH} 5)$ & halogen (> $420 \mathrm{~nm})$ & (280) & - & 155 & 158 & - & - & - & - & - & [193] \\
\hline $\mathrm{Cu}_{2} \mathrm{O}$ & formic $\operatorname{acid}(a q, 2.5 \mathrm{M}, \mathrm{pH} 5)$ & halogen (> $420 \mathrm{~nm})$ & (280) & - & 65 & 64 & - & - & - & - & - & [193] \\
\hline $\mathrm{CdS}$ & formic $\operatorname{acid}(a q, 2.5 \mathrm{M}, \mathrm{pH} 5)$ & halogen (> $420 \mathrm{~nm})$ & (280) & - & 80 & 78 & - & 19 & - & - & - & [193] \\
\hline $\mathrm{CdS}$ & $\mathrm{K}\left[\mathrm{HCO}_{2}\right](a q, 0.5 \mathrm{M})$ & $(>400 \mathrm{~nm})$ & - & - & 80 & - & - & - & - & - & - & {$[101]$} \\
\hline $\mathrm{Pt}(1.5 \%) / \mathrm{CdS}$ & $\mathrm{H}_{2} \mathrm{O} /$ formic $\operatorname{acid}(l, 18: 1)$ & $\mathrm{Hg}(>420 \mathrm{~nm})$ & 400 & - & 1128 & - & - & - & - & - & - & {$[220 \mathrm{~b}]$} \\
\hline $\mathrm{CdS}$ & $\mathrm{H}_{2} \mathrm{O} /$ formic $\operatorname{acid}(l, 18: 1)$ & $\mathrm{Hg}(>420 \mathrm{~nm})$ & 400 & - & 79 & - & - & - & - & - & - & {$[220 \mathrm{~b}]$} \\
\hline $\mathrm{Pt}(0.05 \%) / \mathrm{CdS}$ & $\mathrm{H}_{2} \mathrm{O} /$ formic $\operatorname{acid}(l, 18: 1)$ & $\mathrm{Hg}(>420 \mathrm{~nm})$ & 400 & - & 4460 & - & - & - & - & - & - & [220a] \\
\hline $\mathrm{CdS}$ & $\mathrm{H}_{2} \mathrm{O} /$ formic $\operatorname{acid}(l, 18: 1)$ & $\mathrm{Hg}(>420 \mathrm{~nm})$ & 400 & - & 219 & - & - & - & - & - & - & [220a] \\
\hline $\operatorname{Pt}(3,8 \%)+\mathrm{CdS}$ & formic $\operatorname{acid}(a q, 2 \mathrm{M}, \mathrm{pH} 5)$ & $\mathrm{Hg}(>400 \mathrm{~nm})$ & 500 & - & 385 & 385 & - & 77 & - & - & - & [218] \\
\hline $\operatorname{Pt}(15 \%)+\mathrm{CdS}$ & $\mathrm{H}_{2} \mathrm{O} /$ formic $\operatorname{acid}(l, 7: 1)$ & $\mathrm{Xe}(>320 \mathrm{~nm})$ & 500 & - & 20102 & - & - & - & - & - & - & {$[235]$} \\
\hline $\mathrm{Pt}(15 \%)+\mathrm{CdSe}$ & $\mathrm{H}_{2} \mathrm{O} /$ formic $\operatorname{acid}(l, 7: 1)$ & $\mathrm{Xe}(>320 \mathrm{~nm})$ & 500 & - & 12210 & - & - & - & - & - & - & {$[235]$} \\
\hline $\mathrm{Pt}(0.34 \%) / \mathrm{CdS}(2.5 \%) / \mathrm{Al}-\mathrm{HMS}^{[b]}$ & $\mathrm{H}_{2} \mathrm{O} /$ formic $\operatorname{acid}(l, 4: 1)$ & $\mathrm{Xe}(>420 \mathrm{~nm})$ & 350 & - & 1705 & - & - & - & - & - & - & {$[220 \mathrm{c}]$} \\
\hline $\mathrm{Ru}(0.99 \%) / \mathrm{CdS}(21 \%) / \mathrm{Al}-\mathrm{HMS}^{[b]}$ & $\mathrm{H}_{2} \mathrm{O} /$ formic $\operatorname{acid}(l, 4: 1)$ & $\mathrm{Xe}(>420 \mathrm{~nm})$ & 350 & - & 2753 & - & - & - & - & - & 16.6 & {$[225 \mathrm{c}]$} \\
\hline $\mathrm{Ru}(5 \%) / \mathrm{ZnS}(\mathrm{Cd} / \mathrm{Zn}=4) / \mathrm{CdS}$ & $\mathrm{H}_{2} \mathrm{O} /$ formic $\operatorname{acid}(l, 9: 1)$ & $\mathrm{Xe}(>420 \mathrm{~nm})$ & $(70)$ & - & 5800 & 4520 & - & - & - & - & - & {$[224]$} \\
\hline $\mathrm{ZnS}(\mathrm{Cd} / \mathrm{Zn}=4) / \mathrm{CdS}$ & $\mathrm{H}_{2} \mathrm{O} /$ formic $\operatorname{acid}(l, 9: 1)$ & $\mathrm{Xe}(>420 \mathrm{~nm})$ & $(70)$ & - & 1263 & - & - & - & - & - & - & [224] \\
\hline${ }^{\mathrm{P} 25} \mathrm{TiO}_{2}$ & $\operatorname{acetic} \operatorname{acid}(a q, 1 \mathrm{mM})$ & $\mathrm{Hg}$ & 100 & 25 & - & 7 & - & - & - & - & - & {$[352]$} \\
\hline $\mathrm{Pt}(1-5 \%) / \mathrm{TiO}_{2}$ (anatase) & $\operatorname{AcOH}(l)$ & $\mathrm{Xe}-\mathrm{Hg}$ & 1600 & 55 & - & 1600 & - & - & - & - & - & [89a] \\
\hline $\mathrm{Pt}(1-5 \%) / \mathrm{TiO}_{2}$ (anatase) & $\mathrm{H}_{2} \mathrm{O} /$ acetic $\operatorname{acid}(l, 1: 9)$ & $\mathrm{Xe}-\mathrm{Hg}$ & 1600 & 55 & - & 4060 & - & - & - & - & - & [89a] \\
\hline $\mathrm{Pt}(1-5 \%) / \mathrm{TiO}_{2}$ (anatase) & $\mathrm{H}_{2} \mathrm{O} /$ acetic $\operatorname{acid}(l, 1: 1)$ & $\mathrm{Xe}-\mathrm{Hg}$ & 1600 & 55 & - & 1563 & 1322 & - & 120 & - & - & [89a] \\
\hline $\mathrm{Pt}(3 \%) / \mathrm{TiO}_{2}$ (anatase) & $\mathrm{AcOH} / \mathrm{Na}[\mathrm{AcO}](a q, 4.0: 0.6 \mathrm{M})$ & $\mathrm{Hg}$ (concentrated) & 500 & - & 24 & 65 & 43 & - & 4 & - & - & {$[89 \mathrm{~b}]$} \\
\hline $\mathrm{Pt}(3 \%) / \mathrm{TiO}_{2}$ (rutile) & $\mathrm{AcOH} / \mathrm{Na}[\mathrm{AcO}](a q, 4.0: 0.6 \mathrm{M})$ & Hg (concentrated) & 500 & - & 9 & 11 & 10 & - & 1 & - & - & {$[89 b]$} \\
\hline $\mathrm{Pt}(2 \%) / \mathrm{TiO}_{2}$ (anatase) & $\operatorname{acetic} \operatorname{acid}(v, 11$ Torr $)$ & $\mathrm{Hg}$ & 500 & r.t. & 46 & 132 & 54 & - & 43 & - & - & [315] \\
\hline $\mathrm{Pt}(2 \%) / \mathrm{TiO}_{2}$ (anatase) & $\mathrm{H}_{2} \mathrm{O} /$ acetic $\operatorname{acid}(v, 24: 11$ Torr $)$ & $\mathrm{Hg}$ & 500 & r.t. & 180 & 453 & 104 & - & 180 & - & - & {$[315]$} \\
\hline $\mathrm{Pt}(2 \%) / \mathrm{TiO}_{2}$ (anatase) & $\operatorname{acetic} \operatorname{acid}(l)$ & $\mathrm{Hg}$ & 500 & r.t. & 46 & 290 & 163 & - & 12 & - & - & {$[315]$} \\
\hline $\mathrm{Pt}(0.2 \%))^{/ 25} \mathrm{TiO}_{2}$ & $\mathrm{H}_{2} \mathrm{O} /$ acetic $\operatorname{acid}(l, 9: 1)$ & - & $(1000)$ & - & 66 & - & 92 & - & - & - & - & [119] \\
\hline $\mathrm{Pt}(7 \%) / \mathrm{TiO}_{2}$ (rutile) & $\mathrm{H}_{2} \mathrm{O} / \operatorname{acetic} \operatorname{acid}(l, 6: 1, \mathrm{pH} 2.1)^{[c]}$ & $\begin{array}{l}\mathrm{Hg}(>320 \mathrm{~nm},>320 \\
\mathrm{nm})\end{array}$ & 500 & - & 77 & - & 397 & - & - & - & - & {$[37]$} \\
\hline $\mathrm{Pt}(7 \%) / \mathrm{TiO}_{2}$ (rutile) & $\mathrm{H}_{2} \mathrm{O} /$ acetic $\operatorname{acid}(l, 6: 1, \mathrm{pH} 8.8)^{[c]}$ & $\begin{array}{l}\mathrm{Hg}(>320 \mathrm{~nm},>320 \\
\mathrm{nm})\end{array}$ & 500 & - & 367 & - & 2 & - & - & - & - & {$[37]$} \\
\hline $\mathrm{Pt}(7 \%) / \mathrm{TiO}_{2}$ (anatase) & $\mathrm{Na}[\mathrm{AcO}](a q, 1.7 \% \mathrm{w} / \mathrm{v}, \mathrm{pH} 7.4)^{[c]}$ & $\begin{array}{l}\mathrm{Hg}(>320 \mathrm{~nm},>320 \\
\mathrm{nm})\end{array}$ & 500 & - & 165 & 27 & 0.24 & - & - & {$[d]$} & - & {$[37]$} \\
\hline $\mathrm{Pt}(0.5 \%))^{\mathrm{P25}} \mathrm{TiO}_{2}$ & $\operatorname{acetic} \operatorname{acid}(a q, 0.87 \mathrm{mM})$ & solar simulator & 280 & 40 & 278 & - & - & - & - & - & - & {$[38 \mathrm{a}]$} \\
\hline $\mathrm{Pt}(1 \%) / \mathrm{TiO}_{2}$ & $\operatorname{acetic} \operatorname{acid}(a q, 0.65 \% \mathrm{w} / \mathrm{v})$ & $\mathrm{Hg}$ & 300 & 40 & 28478 & - & - & - & - & - & - & [61] \\
\hline
\end{tabular}


$\mathrm{Pt}(0.6 \%) / \mathrm{TiO}_{2}$ (mesoporous)

$\mathrm{Pt}(0.5 \%) / \mathrm{TiO}_{2}$ (porous)

$\mathrm{TiO}_{2} /$ glass fabric

$\mathrm{Cu}(10 \%)+\mathrm{TiO}$

$\mathrm{Fe}(10 \%) / \mathrm{TiO}_{2}$

${ }^{\mathrm{P} 25} \mathrm{TiO}_{2}$

$\mathrm{Fe}(20 \%) / \mathrm{TiO}_{2}$

$\mathrm{TiO}$

$\mathrm{Eu}(0.02 \% \mathrm{~mol}): \mathrm{TiO}_{2}$

$\mathrm{Sm}(0.05 \% \mathrm{~mol}): \mathrm{TiO}_{2}$

$\mathrm{ZnO}_{2}$

$\mathrm{TiO}_{2}$

$\mathrm{WO}_{3}$

$\mathrm{MgO}$

$\mathrm{SiO}_{2}$

$\gamma-\mathrm{Al}_{2} \mathrm{O}_{3}$

$\mathrm{SiO}_{2}$

$\mathrm{SrTiO}_{3}$

$\mathrm{Pt}(7 \%) / \mathrm{TiO}_{2}$ (rutile)

$\mathrm{Pt}(7 \%) / \mathrm{TiO}_{2}$ (rutile)

$\mathrm{Pt}(7 \%) / \mathrm{TiO}_{2}$ (rutile)

$\mathrm{PtO}_{\mathrm{r}} / \mathrm{Fe}_{2} \mathrm{O}_{3} @ \mathrm{TiO}_{2}$

$\mathrm{Pt}(5 \%) / \mathrm{TaO}_{2.18} \mathrm{Cl}_{0.64}$

$\mathrm{Ce}(4 \% \mathrm{~mol}): \mathrm{Sb}(10 \%): \mathrm{SnO}_{2}$

$\mathrm{Pt}(5 \%) / \mathrm{TiO}_{2}$ (rutile)

$\mathrm{Pt}(5 \%) / \mathrm{CdS}$

$\mathrm{Pt}(1 \%) / \mathrm{CdS}$

$\mathrm{Pt}_{3} \mathrm{Co}(1 \%) / \mathrm{CdS}$

$\mathrm{Co}(1 \%) / \mathrm{CdS}$

$\mathrm{Pt}_{3} \mathrm{Au}(1 \%) / \mathrm{CdS}$

$\mathrm{Pt}_{3} \mathrm{Ni}(1 \%) / \mathrm{CdS}$

$\mathrm{Pt}_{3} \mathrm{Cu}(1 \%) / \mathrm{CdS}$

$\mathrm{Pt}(1 \%) /{ }^{\mathrm{P} 25} \mathrm{TiO}_{2}$

$\mathrm{Pt}_{3} \mathrm{Co}(1 \%) /{ }^{\mathrm{P} 25} \mathrm{TiO}_{2}$

$\mathrm{Pt}_{3} \mathrm{Au}(1 \%) /{ }^{\mathrm{P} 25} \mathrm{TiO}_{2}$

$\mathrm{Pt}_{3} \mathrm{Ni}(1 \%) / /^{\mathrm{P25}} \mathrm{TiO}_{2}$

$\mathrm{Pt}_{3} \mathrm{Cu}(1 \%) /{ }^{\mathrm{P} 25} \mathrm{TiO}_{2}$

$\mathrm{CdS}$

$\mathrm{MoS}_{2}(0.2 \%) / \mathrm{CdS}$

$\mathrm{Pt}(0.2 \%) / \mathrm{CdS}$
$\mathrm{H}_{2} \mathrm{O} / \operatorname{acetic} \operatorname{acid}(l, 10: 1)$

$\mathrm{H}_{2} \mathrm{O} /$ acetic $\operatorname{acid}(l, 4: 1)$

acetic $\operatorname{acid}(a q, 1 \mathrm{M})$

$\operatorname{acetic} \operatorname{acid}(a q, 1 \mathrm{M})$

$\operatorname{acetic} \operatorname{acid}(a q, 1 \mathrm{M})$

$\operatorname{acetic} \operatorname{acid}(a q, 1 \mathrm{M})$

$\operatorname{acetic} \operatorname{acid}(a q, 1 \mathrm{M})$

acetic $\operatorname{acid}(a q, 1 \mathrm{M})$

acetic $\operatorname{acid}(a q, 1 \mathrm{M})$

$\operatorname{acetic} \operatorname{acid}(a q, 1 \mathrm{M})$

$\operatorname{acetic} \operatorname{acid}(v, 665 \mathrm{~Pa})$

$\operatorname{acetic} \operatorname{acid}(v, 665 \mathrm{~Pa})$

acetic $\operatorname{acid}(v, 665 \mathrm{~Pa})$

$\operatorname{acetic} \operatorname{acid}(v, 665 \mathrm{~Pa})$

$\operatorname{acetic} \operatorname{acid}(v, 665 \mathrm{~Pa})$

$\operatorname{acetic} \operatorname{acid}(v, 665 \mathrm{~Pa})$

$\operatorname{acetic} \operatorname{acid}(v)$

acetic $\operatorname{acid}(a q, 1 \mathrm{M})$

$\mathrm{H}_{2} \mathrm{O} /$ propionic $\operatorname{acid}(l, 6: 1)$

$\mathrm{H}_{2} \mathrm{O} /$ butyric $\operatorname{acid}(l, 6: 1)$

$\mathrm{H}_{2} \mathrm{O} /$ valeric $\operatorname{acid}(l, 6: 1)$

$\mathrm{H}_{2} \mathrm{O} /$ lactic $\operatorname{acid}(l, 9: 1)$

$\mathrm{H}_{2} \mathrm{O} /$ lactic $\operatorname{acid}(l, 7: 3)$

$\mathrm{H}_{2} \mathrm{O} /$ lactic $\operatorname{acid}(l, 7: 1)$

$\mathrm{H}_{2} \mathrm{O} /$ lactic $\operatorname{acid}(l, 10: 1)$

$\mathrm{H}_{2} \mathrm{O} /$ lactic $\operatorname{acid}(l, 10: 1)$

$\mathrm{H}_{2} \mathrm{O} /$ lactic $\operatorname{acid}(l, 9: 1)$

$\mathrm{H}_{2} \mathrm{O} /$ lactic $\operatorname{acid}(l, 9: 1)$

$\mathrm{H}_{2} \mathrm{O} /$ lactic $\operatorname{acid}(l, 9: 1)$

$\mathrm{H}_{2} \mathrm{O} /$ lactic $\operatorname{acid}(l, 9: 1)$

$\mathrm{H}_{2} \mathrm{O} /$ lactic $\operatorname{acid}(l, 9: 1)$

$\mathrm{H}_{2} \mathrm{O} /$ lactic $\operatorname{acid}(l, 9: 1)$

$\mathrm{H}_{2} \mathrm{O} /$ lactic $\operatorname{acid}(l, 9: 1)$

$\mathrm{H}_{2} \mathrm{O} /$ lactic $\operatorname{acid}(l, 9: 1)$

$\mathrm{H}_{2} \mathrm{O} /$ lactic $\operatorname{acid}(l, 9: 1)$

$\mathrm{H}_{2} \mathrm{O} /$ lactic acid $(l, 9: 1)$

$\mathrm{H}_{2} \mathrm{O} /$ lactic $\operatorname{acid}(l, 9: 1)$

$\mathrm{H}_{2} \mathrm{O} /$ lactic $\operatorname{acid}(l, 9: 1)$

$\mathrm{H}_{2} \mathrm{O} /$ lactic $\operatorname{acid}(l, 9: 1)$

$\mathrm{H}_{2} \mathrm{O} /$ lactic $\operatorname{acid}(l, 9: 1)$

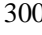

$\mathrm{Hg}$

$\mathrm{Hg}$

$\mathrm{Hg}$

$\mathrm{Hg}$

$\mathrm{Hg}-\mathrm{Xe}$

$\mathrm{Hg}-\mathrm{Xe}$

$\mathrm{Hg}-\mathrm{Xe}$

$\mathrm{Hg}-\mathrm{Xe}$

$\mathrm{Hg}-\mathrm{Xe}$

$\mathrm{Hg}-\mathrm{Xe}$

$\mathrm{Hg}-\mathrm{Xe}$

$\mathrm{Hg}$

$\mathrm{Hg}$ (> $320 \mathrm{~nm},>320$

$\mathrm{nm})$

$\mathrm{Hg}$ (> $320 \mathrm{~nm},>320$

$\mathrm{nm})$

$\mathrm{Hg}(>320 \mathrm{~nm},>320$

$\mathrm{nm})$

$\mathrm{Xe}(>420 \mathrm{~nm})$

$\mathrm{Xe}$

$\mathrm{Xe}(>320 \mathrm{~nm})$

$\mathrm{Xe}$

Xe (> $420 \mathrm{~nm})$

$\mathrm{Xe}(>420 \mathrm{~nm})$

$\mathrm{Xe}(>420 \mathrm{~nm})$

$\mathrm{Xe}(>420 \mathrm{~nm})$

$\mathrm{Xe}(>420 \mathrm{~nm})$

$\mathrm{Xe}(>420 \mathrm{~nm})$

$\mathrm{Xe}(>420 \mathrm{~nm})$

$\mathrm{Xe}(>420 \mathrm{~nm})$

$\mathrm{Xe}(>420 \mathrm{~nm})$

$\mathrm{Xe}(>420 \mathrm{~nm})$

$\mathrm{Xe}(>420 \mathrm{~nm})$

$\mathrm{Xe}(>420 \mathrm{~nm})$

$\mathrm{Xe}(>420 \mathrm{~nm})$

$\mathrm{Xe}(>420 \mathrm{~nm})$
1000

$\begin{array}{rrr}1 & 114 & 164 \\ 144 & 640 & 590 \\ 7 & 102 & 93 \\ 2 & 141 & 50 \\ 7 & 257 & 260 \\ 8 & 100 & 107 \\ 9 & 88 & 108 \\ 3 & 131 & 124 \\ - & 0.098 & 0.034 \\ - & 0.370 & 0.018 \\ - & 0.026 & 0.003 \\ - & 0.923 & 0.179 \\ - & 0.336 & 0.168 \\ - & 0.336 & 0.086 \\ - & 0.687 & 1.307\end{array}$

13

51

111

174

1100

1500

1008
1000

8170

15860

1070

14900

12810

3890

690

1040

890
820

150

5400

4400
- [140b]

[353c]

- [406]

- [353b]

- [353b]

- [333

- [353a]

- [168]

- $[168$

- $[44 a]$

- [44a]

- [44a]

- [44a]

- [44a]

- $\quad[44 a]$

- [44b]

- [201d]

- [37]

- [37]

[37]

- [407]

- [408]

- [188]

$[g] \quad 71^{[h]} \quad[84]$

$667^{[i]} \quad 38^{[j]} \quad[84]$

- [217]

- [217]

- [217]

- [217]

- [217]

- [217]

- [217]

- [217]

- [217]

- [217]

- [217]

- [291b]

- [291b]

- [291b] 
$\mathrm{H}_{2} \mathrm{O} /$ lactic $\operatorname{acid}(l, 9: 1)$

$\mathrm{Au}(0.2 \%) / \mathrm{CdS}$

$\mathrm{Xe}(>420 \mathrm{~nm})$

$\mathrm{WS}_{2}(1 \%) / \mathrm{CdS}$

$\mathrm{H}_{2} \mathrm{O} /$ lactic $\operatorname{acid}(l, 9: 1)$

$\mathrm{H}_{2} \mathrm{O} /$ lactic $\operatorname{acid}(l, 9: 1)$

$\mathrm{Pt}(1 \%) / \mathrm{CdS}$

$\mathrm{H}_{2} \mathrm{O} /$ lactic $\operatorname{acid}(l, 9: 1)$

$\mathrm{H}_{2} \mathrm{O} /$ lactic $\operatorname{acid}(l, 9: 1)$

$\mathrm{H}_{2} \mathrm{O} /$ lactic $\operatorname{acid}(l, 9: 1)$

$\mathrm{Au}(1 \%) / \mathrm{CdS}$

$\mathrm{NiS}(1.2 \% \mathrm{~mol}) / \mathrm{CdS}$

$\mathrm{H}_{2} \mathrm{O} /$ lactic $\operatorname{acid}(l, 9: 1)$

$\mathrm{H}_{2} \mathrm{O} /$ lactic $\operatorname{acid}(l, 7: 3)$

$\mathrm{H}_{2} \mathrm{O} /$ lactic $\operatorname{acid}(l, 7: 3)$

$\mathrm{H}_{2} \mathrm{O} /$ lactic $\operatorname{acid}(l, 7: 3)$

$\mathrm{Pt}(1 \% \mathrm{~mol}) / \mathrm{CdS}$

$\mathrm{H}_{2} \mathrm{O} /$ lactic $\operatorname{acid}(l, 7: 3)$

$\mathrm{H}_{2} \mathrm{O} /$ lactic $\operatorname{acid}(l, 9: 1)$

$\mathrm{H}_{2} \mathrm{O} /$ lactic $\operatorname{acid}(l, 9: 1)$

$\mathrm{H}_{2} \mathrm{O} /$ lactic $\operatorname{acid}(l, 9: 1)$

$\mathrm{H}_{2} \mathrm{O} /$ lactic $\operatorname{acid}(l, 9: 1)$

$\mathrm{H}_{2} \mathrm{O} /$ lactic $\operatorname{acid}(l, 9: 1)$

$\mathrm{H}_{2} \mathrm{O} /$ lactic $\operatorname{acid}(l, 9: 1)$

$\mathrm{H}_{2} \mathrm{O} /$ lactic $\operatorname{acid}(l, 9: 1)$

$\mathrm{H}_{2} \mathrm{O} /$ lactic $\operatorname{acid}(l, 9: 1)$

$\mathrm{H}_{2} \mathrm{O} /$ lactic $\operatorname{acid}(l, 9: 1)$

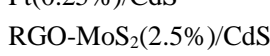

$\mathrm{H}_{2} \mathrm{O} /$ lactic $\operatorname{acid}(l, 4: 1)$

$\mathrm{CdS} /\left\{\mathrm{MoS}_{2} / \mathrm{RGO}(\mathrm{Mo} / \mathrm{C}=2)\right\}(2 \%)$

$\mathrm{MoS}_{2}(1.5 \%) / \mathrm{CdS} / \mathrm{RGO}(1.5 \%)$

$\mathrm{H}_{2} \mathrm{O} /$ lactic $\operatorname{acid}(l, 9: 1)$

$\mathrm{MoS}_{2}(0.2 \%) / g-\mathrm{C}_{3} \mathrm{~N}_{4}$ (mesoporous)

$\mathrm{Pt}(2 \%) / g-\mathrm{C}_{3} \mathrm{~N}_{4}$ (mesoporous)

$\mathrm{H}_{2} \mathrm{O} /$ lactic $\operatorname{acid}(l, 9: 1)$

$\mathrm{H}_{2} \mathrm{O} /$ lactic $\operatorname{acid}(l, 9: 1)$

$\mathrm{H}_{2} \mathrm{O} /$ lactic $\operatorname{acid}(l, 9: 1)$

$\mathrm{H}_{2} \mathrm{O} /$ lactic $\operatorname{acid}(l, 20: 3)$

$\mathrm{H}_{2} \mathrm{O} /$ lactic $\operatorname{acid}(l, 20: 3)$

$\mathrm{H}_{2} \mathrm{O} /$ lactic $\operatorname{acid}(l, 4: 1)$

$\mathrm{H}_{2} \mathrm{O} /$ lactic $\operatorname{acid}(l, 4: 1)$

$\mathrm{H}_{2} \mathrm{O} /$ lactic $\operatorname{acid}(l, 4: 1)$

$\mathrm{H}_{2} \mathrm{O} /$ lactic $\operatorname{acid}(l, 9: 1)$

$\mathrm{H}_{2} \mathrm{O} /$ lactic $\operatorname{acid}(l, 19: 1, \mathrm{pH} 3)^{[c]}$

$\mathrm{H}_{2} \mathrm{O} /$ lactic $\operatorname{acid}(l, 19: 1, \mathrm{pH} 3)^{[c]}$

$\mathrm{H}_{2} \mathrm{O} /$ lactic $\operatorname{acid}(l, 19: 1, \mathrm{pH} 3)^{[c]}$

$\mathrm{H}_{2} \mathrm{O} /$ lactic $\operatorname{acid}(l, 10: 1)$

$\mathrm{H}_{2} \mathrm{O} /$ lactic $\operatorname{acid}(l, 9: 1)$

$\mathrm{H}_{2} \mathrm{O} /$ lactic $\operatorname{acid}(l, 9: 1)$

$\mathrm{H}_{2} \mathrm{O} /$ lactic $\operatorname{acid}(l, 9: 1)$

oxalic $\operatorname{acid}(a q, 0.01 \mathrm{M})$

oxalic $\operatorname{acid}(a q, 4.9 \mathrm{mM})$

oxalic $\operatorname{acid}(a q, 1 \mathrm{mM})$

$\mathrm{Xe}(>420 \mathrm{~nm})$

$\mathrm{Xe}(>420 \mathrm{~nm})$

$\mathrm{Xe}(>420 \mathrm{~nm})$

$\mathrm{Xe}(>420 \mathrm{~nm})$

$\mathrm{Xe}(>420 \mathrm{~nm})$

$\mathrm{Xe}(>420 \mathrm{~nm})$

$\mathrm{Xe}(>420 \mathrm{~nm})$

$\mathrm{Xe}(>420 \mathrm{~nm})$

$\mathrm{Xe}(>420 \mathrm{~nm})$

$\mathrm{Xe}(>420 \mathrm{~nm})$

$\mathrm{Xe}(>420 \mathrm{~nm})$

$\mathrm{Xe}(>420 \mathrm{~nm})$

$\mathrm{X}$

$\mathrm{Xe}$

$\mathrm{Xe}(>420 \mathrm{~nm})$

$\mathrm{Xe}(>420 \mathrm{~nm})$

$\mathrm{Xe}(>420 \mathrm{~nm})$

$\mathrm{Xe}(>420 \mathrm{~nm})$

$\mathrm{Xe}(>420 \mathrm{~nm})$

$\mathrm{Xe}(>420 \mathrm{~nm})$

$\mathrm{Xe}(>420 \mathrm{~nm})$

$\mathrm{Xe}(>420 \mathrm{~nm})$

$\mathrm{Xe}(>420 \mathrm{~nm})$

$\operatorname{LED}(>420 \mathrm{~nm})$

$\operatorname{LED}(>420 \mathrm{~nm})$

Xe $(>420 \mathrm{~nm})$

$\mathrm{Xe}(>420 \mathrm{~nm})$

$\mathrm{Xe}(>420 \mathrm{~nm})$

$\mathrm{Xe}(>420 \mathrm{~nm})$

LED (> $420 \mathrm{~nm}$ )

LED (> $420 \mathrm{~nm})$

LED (> $420 \mathrm{~nm}$ )

visible (> $420 \mathrm{~nm}$ )

$\mathrm{Xe}(>420 \mathrm{~nm})$

$\mathrm{Xe}(>420 \mathrm{~nm})$

$\mathrm{Xe}(>420 \mathrm{~nm})$ 
$\mathrm{Pt}(2 \%) / \mathrm{TiO}_{2}$

$\mathrm{Pt}(5 \%) / \mathrm{TiO}_{2}$ (rutile)

$\mathrm{Pt}(5 \%) / \mathrm{CdS}$

$\mathrm{Pt}(5 \%) / \mathrm{TiO}_{2}$

$\mathrm{Pt}(5 \%) / \mathrm{WO}_{3}$

$\mathrm{Pt}(5 \%) / \mathrm{Fe}_{2} \mathrm{O}_{3}$

$\mathrm{Pt}(5 \%) / \mathrm{MoS}_{2}$

$\mathrm{Pt}(2 \%) / \mathrm{TiO}_{2}$

$\mathrm{Pt}(5 \%) / \mathrm{TiO}_{2}$

$\mathrm{Pt}(2 \%) / \mathrm{TiO}_{2}$

$\mathrm{H}_{2} \mathrm{O} /$ glycolic $(0.51 \mathrm{~g} / \mathrm{L})$
$\mathrm{H}_{2} \mathrm{O} /$ glycolic $(1: 10 \mathrm{v} / \mathrm{v})$
$\mathrm{H}_{2} \mathrm{O} /$ glycolic $(1: 10 \mathrm{v} / \mathrm{v})$
$\mathrm{H}_{2} \mathrm{O} /$ pyruvic $\operatorname{acid}(l, 1: 1)$
$\mathrm{H}_{2} \mathrm{O} /$ pyruvic $\operatorname{acid}(l, 1: 1)$
$\mathrm{H}_{2} \mathrm{O} /$ pyruvic $\operatorname{acid}(l, 1: 1)$
$\mathrm{H}_{2} \mathrm{O} /$ pyruvic $\operatorname{acid}(l, 1: 1)$
gluconic $\operatorname{acid}\left(a q, 1.3 \mathrm{~g} \mathrm{~L}^{-1}\right)$
stearic $\operatorname{acid}(a q, 1.7 \% \mathrm{w} / \mathrm{v})$

$\begin{array}{ll}\mathrm{Hg} & 100 \\ \mathrm{Xe} & 500 \\ \mathrm{Xe} & 500 \\ \mathrm{Xe} & 500 \\ \mathrm{Xe} & 500 \\ \mathrm{Xe} & 500 \\ \mathrm{Xe} & 500 \\ \mathrm{Hg} & 100 \\ \mathrm{Xe} & 500 \\ \mathrm{Hg} & 100\end{array}$

100
500
500
500
500
500
500
100
500
100

20
-
r.
r.t.
r.t.
r.
20
r.t.

r.t.

$\begin{array}{rr}284 & 109 \\ 105 & 76 \\ 392 & 3 \\ 110 & 813 \\ 0 & 207 \\ 0 & 197 \\ 0 & 383 \\ 675 & 296 \\ 29 & - \\ 1989 & 509\end{array}$

$$
\begin{aligned}
& \begin{array}{rrrrr}
- & - & - & - & {[39 \mathrm{~b}]} \\
- & - & {[n]} & - & {[84]}
\end{array} \\
& \text { - r.t. }
\end{aligned}
$$

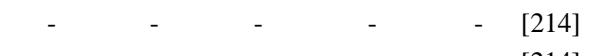

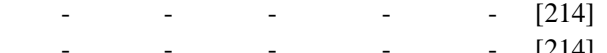

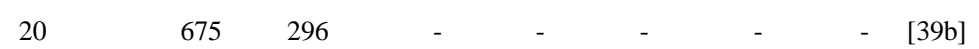

$$
\begin{aligned}
& \text { - }[108 \mathrm{a}] \\
& \text { - [39b] }
\end{aligned}
$$$$
\left.\begin{array}{llllllll}
\text { r.t. } & 110 & 813 & - & - & - & - & -
\end{array}\right]
$$$$
20
$$

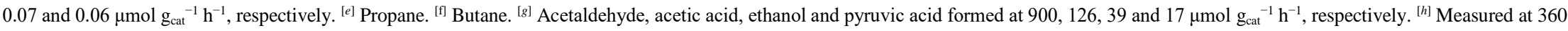

\begin{tabular}{|c|c|c|c|c|c|c|c|c|c|c|}
\hline \multirow[b]{2}{*}{ photocatalyst } & \multirow[b]{2}{*}{ reaction medium ${ }^{[a]}$} & \multirow[b]{2}{*}{ light source } & \multirow{2}{*}{$\begin{array}{l}P / \mathrm{W} \\
\left(I / \mathrm{mW} \mathrm{cm}^{-2}\right)\end{array}$} & \multirow[b]{2}{*}{$T /{ }^{\circ} \mathrm{C}$} & \multicolumn{4}{|c|}{ production rates $/ \mu \mathrm{mol} \mathrm{g}_{\mathrm{cat}}{ }^{-1} \mathrm{~h}^{-1}$} & \multirow[b]{2}{*}{$\Phi_{a} / \%$} & \multirow[b]{2}{*}{ reference } \\
\hline & & & & & $\mathrm{H}_{2}$ & $\mathrm{CO}_{2}$ & $\mathrm{CH}_{4}$ & $\mathrm{CO}$ & & \\
\hline $\mathrm{Pt}(5 \%) / \mathrm{TiO}_{2}$ & glucose $(a q, 1.7 \%)$ & $\mathrm{Xe}$ & 500 & r.t. & 377 & - & - & - & - & [108a] \\
\hline$\left.\mathrm{Pt}(0.5 \%)\right|^{\mathrm{P} 25} \mathrm{TiO}_{2}$ & glucose $(a q, 0.417 \mathrm{mM})$ & solar simulator & 300 & 40 & 848 & 308 & - & - & - & [317] \\
\hline $\mathrm{Pt}(0.5 \%) /{ }^{\mathrm{P} 25} \mathrm{TiO}_{2}$ & glucose $(a q, 1 \%)$ & solar simulator & 300 & 40 & 2400 & - & - & - & - & [317] \\
\hline$\left.\mathrm{Pt}(0.5 \%)\right|^{\mathrm{P} 25} \mathrm{TiO}_{2}$ & $\operatorname{glucose}(a q, 0.06 \mathrm{M})$ & - & - & - & 680 & - & - & - & - & [62b] \\
\hline $\mathrm{Pt}(0.3 \%) /{ }^{\mathrm{P25}} \mathrm{TiO}_{2}$ & $\operatorname{glucose}(a q, 0.46 \mathrm{mM}, \mathrm{pH} 10)$ & UVA (366 nm) & $15 \times 2$ & - & 307 & - & - & - & 1.1 & [325] \\
\hline $\mathrm{Pt}(0.5 \%) /{ }^{\mathrm{P} 25} \mathrm{TiO}_{2}$ & $\operatorname{glucose}(a q, 5.3 \mathrm{mM})$ & UVA $(366 \mathrm{~nm})$ & $15 \times 4$ & - & 357 & - & - & - & - & [321] \\
\hline $\mathrm{Pt}(2 \%) / \mathrm{TiO}_{2}$ & $\operatorname{glucose}\left(a q, 1.2 \mathrm{~g} \mathrm{~L}^{-1}\right)$ & $\mathrm{Hg}$ & 100 & 20 & 941 & 433 & - & - & - & [39b] \\
\hline $\mathrm{Pt}(0.1 \%) / \mathrm{TiO}_{2}(74 \%$ rutile $)$ & $\operatorname{glucose}(a q, 20 \%)$ & $\mathrm{Xe}$ & 300 & - & 749 & - & - & - & - & [94b] \\
\hline $\mathrm{Pt} / \mathrm{TiO}_{2}$ (anatase) & glucose $(a q, 33 \%)^{[b]}$ & $\mathrm{Hg}$ & $(7)^{[c]}$ & 60 & 453 & 47 & - & - & - & [357] \\
\hline $\mathrm{Pt} /(\mathrm{B}, \mathrm{N}): \mathrm{TiO}_{2}$ & glucose $(a q, 5 \%)$ & $\mathrm{Xe}$ & 300 & - & 2933 & - & - & - & - & [359] \\
\hline $\mathrm{Pt}(2 \%) / \mathrm{F}: \mathrm{TiO}_{2}$ (nanosheets) & $\operatorname{glucose}(a q, 0.5 \mathrm{M})$ & $\mathrm{Xe}$ & (20) & - & 6680 & - & - & - & - & [121a] \\
\hline $\mathrm{Pd}(0.5 \%) / \mathrm{TiO}_{2}$ & glucose $(a q, 0.5 \%)$ & $\mathrm{Xe}$ & 400 & - & 569 & - & - & - & - & [90a] \\
\hline $\mathrm{Pt}(1 \%) / \mathrm{TiO}_{2}$ & glucose $(a q, 0.05 \%)$ & $\mathrm{Hg}$ & 125 & 30 & 3556 & - & - & - & - & [410] \\
\hline $\mathrm{Pd}(1 \%) / \mathrm{TiO}_{2}$ & glucose $(a q, 0.05 \%)$ & $\mathrm{Hg}$ & 125 & 30 & 2630 & - & - & - & - & [410] \\
\hline $\mathrm{Au}(1 \%) / \mathrm{TiO}_{2}$ (anatase) & $\mathrm{H}_{2} \mathrm{O} / \operatorname{glucose}(l, 5: 2 \mathrm{w} / \mathrm{w})$ & $\mathrm{Xe}$ & 300 & 25 & 1600 & - & - & - & - & [326] \\
\hline $\mathrm{Pt}(1 \%) / \mathrm{TiO}_{2}$ & glucose $(a q, 0.025 \%)$ & $\mathrm{Hg}$ & 125 & 30 & 4100 & - & - & - & - & [62a] \\
\hline $\mathrm{Pd}(1 \%) / \mathrm{TiO}_{2}$ & glucose $(a q, 0.025 \%)$ & $\mathrm{Hg}$ & 125 & 30 & 4500 & - & - & - & - & [62a] \\
\hline $\mathrm{Rh}(1 \%) / \mathrm{TiO}_{2}$ & glucose $(a q, 0.025 \%)$ & $\mathrm{Hg}$ & 125 & 30 & 2100 & - & - & - & - & [62a] \\
\hline $\mathrm{Au}(1 \%) / \mathrm{TiO}_{2}$ & $\operatorname{glucose}(a q, 0.025 \%)$ & $\mathrm{Hg}$ & 125 & 30 & 1950 & - & - & - & - & [62a] \\
\hline $\mathrm{Ag}(1 \%) / \mathrm{TiO}_{2}$ & $\operatorname{glucose}(a q, 0.025 \%)$ & $\mathrm{Hg}$ & 125 & 30 & 400 & - & - & - & - & [62a] \\
\hline $\mathrm{Ru}(1 \%) / \mathrm{TiO}_{2}$ & glucose $(a q, 0.025 \%)$ & $\mathrm{Hg}$ & 125 & 30 & 400 & - & - & - & - & [62a] \\
\hline $\mathrm{TiO}_{2}$ & glucose $(a q, 0.025 \%)$ & $\mathrm{Hg}$ & 125 & 30 & 200 & - & - & - & - & [62a] \\
\hline $\mathrm{Rh}(0.3 \%) / /^{\mathrm{P} 25} \mathrm{TiO}_{2}$ & $\operatorname{glucose}(a q, 1.25 \mathrm{mM})$ & $\mathrm{Hg}$ & 300 & - & 1440 & - & - & 4 & - & [95] \\
\hline
\end{tabular}
metal organic framework. [ $n$ ] Formaldehyde, glyoxylic acid and methanol formed at 31, 24 and $<0.5 \mu \mathrm{mol} \mathrm{g}_{\mathrm{cat}}{ }^{-1} \mathrm{~h}^{-1}$, respectively. [o] Glyoxylic acid.

Table 7. Selected Photoreforming Data for Saccharides 
$\mathrm{Cu}(0.3 \%) /{ }^{\mathrm{P} 25} \mathrm{TiO}_{2}$

$\mathrm{Pt}(0.3 \%) /{ }^{\mathrm{P} 25} \mathrm{TiO}_{2}$

$\mathrm{Ni}(0.3 \%) / /^{\mathrm{P} 25} \mathrm{TiO}_{2}$

$\mathrm{Au}(0.3 \%) /{ }^{\mathrm{P} 25} \mathrm{TiO}_{2}$

$\mathrm{Ru}(0.3 \%) /{ }^{\mathrm{P} 25} \mathrm{TiO}_{2}$

$\operatorname{Ir}(0.3 \%) / /^{\mathrm{P} 25} \mathrm{TiO}_{2}$

${ }^{225} \mathrm{TiO}_{2}$

$\mathrm{Pd}_{\text {shell }} \mathrm{Au}_{\text {core }}(1 \%){ }^{\mathrm{P} 25} \mathrm{TiO}_{2}$

$\mathrm{Fe}(20 \%) / \mathrm{TiO}_{2}$

$\mathrm{Ni}(5 \%) / \mathrm{TiO}_{2}-\mathrm{SiO}_{2}$

$\mathrm{Sn}(0.24 \%) / \mathrm{RuO}_{2}(0.68 \%) /{ }^{225} \mathrm{TiO}_{2}$

$\mathrm{Pt}(1 \%) /\left(\mathrm{CNT}+\mathrm{TiO}_{2}\right)$

$\beta-\mathrm{Fe}_{2} \mathrm{O}_{3}$ (layer)

$\varepsilon-\mathrm{Fe}_{2} \mathrm{O}_{3}$ (layer)

$\mathrm{Pt}(0.5 \%) / \mathrm{SrTiO}_{3}$

$\mathrm{NiO}(0.2 \%) / \mathrm{La}\left(2 \%\right.$ vs. Ta): $\mathrm{NaTaO}_{3}$

$\mathrm{Pt}(0.2 \%) / \mathrm{La}\left(2 \%\right.$ vs. Ta) $: \mathrm{NaTaO}_{3}$

$\mathrm{Pt}(1 \%) / \mathrm{Bi}_{0.5} \mathrm{Y}_{0.5} \mathrm{VO}_{4}$

$\mathrm{Pt}(0.5 \%) / \mathrm{ZnS}(17 \%)-\mathrm{ZnIn}_{2} \mathrm{~S}_{4}$

$\mathrm{Pt}(0.5 \%) / \mathrm{Cd}_{0.5} \mathrm{Zn}_{0.5} \mathrm{~S}$

$\mathrm{Pt}(0.5 \%))^{\mathrm{P} 25} \mathrm{TiO}_{2}$

$\mathrm{Pt}(0.5 \%))^{/ 25} \mathrm{TiO}_{2}$

$\mathrm{Pt}(1 \%) /\left(\mathrm{CNT}+\mathrm{TiO}_{2}\right)$

$\mathrm{Pd}_{\text {shell }} \mathrm{Au}_{\text {core }}(1 \%) /{ }^{\mathrm{P} 25} \mathrm{TiO}$

$\mathrm{Pt}(0.5 \%) /{ }^{225} \mathrm{TiO}_{2}$

$\mathrm{Pt}(0.5 \%))^{225} \mathrm{TiO}_{2}$

$\mathrm{Pt}(2 \%) / \mathrm{TiO}_{2}$

$\mathrm{Pt}(2 \%) /{ }^{\mathrm{P} 25} \mathrm{TiO}_{2}$

$\mathrm{Pt}(0.5 \%) /{ }^{\mathrm{P} 25} \mathrm{TiO}_{2}$

$\mathrm{Pt}(1 \%) / \mathrm{TiO}_{2}$ (anatase)

$\mathrm{Pt}(0.5 \%) /{ }^{\mathrm{P2} 5} \mathrm{TiO}_{2}$

$\mathrm{Pt}(1 \%) / \mathrm{TiO}_{2}$

$\mathrm{Pt}(5 \%) / \mathrm{TiO}_{2}$

$\mathrm{Pt}(0.5 \%))^{\mathrm{P25}} \mathrm{TiO}_{2}$

$\mathrm{Pt}(0.3 \%))^{/ 25} \mathrm{TiO}_{2}$

$\mathrm{RuO}_{2} / \mathrm{Pt} / \mathrm{TiO}_{2}$

$\mathrm{RuO}_{2} / \mathrm{Pt} / \mathrm{TiO}_{2}$

$\mathrm{TiO}_{2}$

$\mathrm{Pd}(0.5 \%) / \mathrm{TiO}_{2}$

$\mathrm{V}(1 \%) / \mathrm{TiO}_{2}(5 \%) / \mathrm{SiO}_{2}$

$\mathrm{Au}(1 \%) / \mathrm{TiO}_{2}(5 \%) / \mathrm{SiO}_{2}$
$\mathrm{H}_{2} \mathrm{O} /$ glucose $(1.25 \mathrm{mM})$

$\mathrm{H}_{2} \mathrm{O}$ /glucose (1.25 mM)

$\mathrm{H}_{2} \mathrm{O} /$ glucose $(1.25 \mathrm{mM})$

$\mathrm{H}_{2} \mathrm{O} /$ glucose $(1.25 \mathrm{mM})$

$\mathrm{H}_{2} \mathrm{O}$ /glucose (1.25 mM)

$\mathrm{H}_{2} \mathrm{O} /$ glucose $(1.25 \mathrm{mM})$

$\mathrm{H}_{2} \mathrm{O} /$ glucose $(1.25 \mathrm{mM})$

glucose $(a q, 25 \%$ w/w)

glucose $(a q, 1 \mathrm{M})$

glucose $\left(a q, 1.25 \mathrm{~g} \mathrm{~L}^{-1}\right)$

glucose $(a q, 0.06 \%)$

glucose $(a q, 0.02 \mathrm{M})$

glucose $(a q, 0.055 \mathrm{M})$

glucose $(a q, 0.055 \mathrm{M})$

glucose $(a q, 1.5 \mathrm{M})$

glucose $(a q, 0.03 \%)$

glucose $(a q, 0.03 \%)$

glucose $(a q, 0.1 \mathrm{M})$

glucose $(a q, 0.1 \mathrm{M})^{[d]}$

glucose $(a q, 0.05 \mathrm{M})^{[e]}$

fructose $(a q, 0.204 \mathrm{mM})$

fructose $(a q, 1 \%)$

fructose $(a q, 0.02 \mathrm{M})$

fructose $(a q, 25 \% \mathrm{w} / \mathrm{w})$

galactose $(a q, 1 \mathrm{M})$

mannose $(a q, 1 \%)$

xylose $\left(a q, 1.0 \mathrm{~g} \mathrm{~L}^{-1}\right)$

xylose $(a q, 8.4 \mathrm{mM})^{[f]}$

arabinose $(a q, 1 \%)$

arabinose $(a q, 0.02 \mathrm{M})$

ribose $(a q, 1 \%)$

sucrose $(a q, 0.025 \%)$

sucrose $(a q, 1.7 \%)$

sucrose $(a q, 0.06 \mathrm{M})$

$\operatorname{sucrose}(a q, 0.22 \mathrm{mM})^{[g]}$

sucrose $\left(a q, 0.325 \mathrm{~g} \mathrm{~L}^{-1}\right)$

sucrose $\left(a q, 15 \mathrm{~g} \mathrm{~L}^{-1}\right)$

sucrose $\left(a q, 15 \mathrm{~g} \mathrm{~L}^{-1}\right)$

sucrose $(a q, 0.5 \%)$

sucrose $(a q, 10 \%)$

sucrose $(a q, 10 \%)$

\section{$\mathrm{Hg}$}

$\mathrm{Hg}$

$\mathrm{Hg}$

$\mathrm{Hg}$

UV LED (365 nm)

$\mathrm{Hg}$

$\mathrm{Hg}$

$\mathrm{Hg}$

$\mathrm{Hg}$

Xe (solar, > $300 \mathrm{~nm}$ )

Xe (solar, > $300 \mathrm{~nm}$ )

$\mathrm{Hg}$

$\mathrm{Hg}$

metal-halogen $(>420 \mathrm{~nm})$

$\mathrm{Hg}(>420 \mathrm{~nm})$

Xe (solar)

Xe (solar)

$\mathrm{Hg}$

UV LED (365 nm)

solar

Xe (solar)

$\mathrm{Hg}$

$\mathrm{Hg}$

Xe (solar)

$\mathrm{Hg}$

Xe (solar)

$\mathrm{Hg}$

$\mathrm{Xe}$

UVA $(366 \mathrm{~nm})$

$\mathrm{Hg}$

$\mathrm{Xe}$

$\mathrm{Xe}$

$\mathrm{Ne}$

$\mathrm{Ne}$

\begin{tabular}{|c|c|c|c|c|c|c|c|}
\hline 300 & - & 1080 & - & - & 32 & - & [95] \\
\hline 300 & - & 1000 & - & - & 8 & - & [95] \\
\hline 300 & - & 980 & - & - & 10 & - & [95] \\
\hline 300 & - & 600 & - & - & 3 & - & [95] \\
\hline 300 & - & 400 & - & - & 1 & - & [95] \\
\hline 300 & - & 300 & - & - & 2 & - & [95] \\
\hline 300 & - & 20 & - & - & 5 & - & [95] \\
\hline (60) & - & 8865 & - & - & - & - & [287] \\
\hline- & 25 & 2 & 4 & 1 & - & - & [379] \\
\hline 300 & r.t. & 1300 & - & - & - & - & [411] \\
\hline 125 & 20 & 2444 & - & 15 & 600 & 0.5 & [283] \\
\hline 150 & 25 & 99 & - & - & - & - & [159a] \\
\hline 150 & - & 2484 & - & - & - & - & [196a] \\
\hline 150 & - & 4259 & - & - & - & - & [196a] \\
\hline $16 \times 176$ & 15 & 210 & - & - & - & - & [201a] \\
\hline 125 & 30 & 14200 & - & - & - & - & [206] \\
\hline 125 & 30 & 8660 & - & - & - & - & [206] \\
\hline 350 & - & 51 & - & - & - & - & {$[102]$} \\
\hline 400 & $\mathrm{rt}$ & 147 & - & - & - & - & [58] \\
\hline 400 & $\mathrm{rt}$ & 301 & - & - & - & - & [63] \\
\hline 300 & 80 & 900 & - & - & - & - & [317] \\
\hline 300 & 40 & 1950 & - & - & - & - & [317] \\
\hline 150 & 25 & 51 & - & - & - & - & [159a] \\
\hline (60) & - & 6065 & - & - & - & - & [287] \\
\hline 450 & 40 & 4200 & - & - & - & - & [9] \\
\hline 300 & 40 & 1725 & - & - & - & - & [317] \\
\hline 100 & 20 & 2567 & 786 & - & - & - & [39b] \\
\hline 100 & - & 725 & 363 & - & - & - & {$[39 c]$} \\
\hline 300 & 40 & 2625 & - & - & - & - & [317] \\
\hline 150 & 25 & 105 & - & - & - & - & [159a] \\
\hline 300 & 40 & 2175 & - & - & - & - & [317] \\
\hline 125 & 30 & 3100 & - & - & - & - & [62a] \\
\hline 500 & r.t. & 307 & - & - & - & - & [108a] \\
\hline - & - & 450 & - & - & - & - & {$[62 \mathrm{~b}]$} \\
\hline $15 \times 2$ & - & 233 & - & - & - & 0.8 & [325] \\
\hline 500 & - & 833 & 417 & - & - & - & [36] \\
\hline 500 & - & 47 & 22 & - & - & - & [36] \\
\hline 500 & - & 0.3 & - & - & - & - & [36] \\
\hline 400 & - & 391 & - & - & - & - & [90a] \\
\hline 200 & - & 116 & 48 & - & - & - & [358] \\
\hline 200 & - & 113 & 52 & - & - & - & [358] \\
\hline
\end{tabular}




\begin{tabular}{|c|c|c|c|c|c|c|c|c|c|}
\hline $\mathrm{Mn}(1 \%) / \mathrm{TiO}_{2}(5 \%) / \mathrm{SiO}_{2}$ & $\operatorname{sucrose}(a q, 10 \%)$ & $\mathrm{Ne}$ & 200 & - & 112 & 47 & - & - & $-\quad[358]$ \\
\hline $\mathrm{N}(1 \%)-\mathrm{S}(1 \%): \mathrm{TiO}_{2}$ & $\operatorname{sucrose}(a q, 10 \%)$ & $\mathrm{Ne}$ & 200 & - & 114 & 55 & - & - & $-\quad[358]$ \\
\hline $\mathrm{TiO}_{2}(5 \%) / \mathrm{SiO}_{2}$ & $\operatorname{sucrose}(a q, 10 \%)$ & $\mathrm{Ne}$ & 200 & - & 27 & - & - & - & $-\quad[358]$ \\
\hline $\mathrm{Pt} /(\mathrm{B}, \mathrm{N}): \mathrm{TiO}_{2}$ & $\operatorname{sucrose}(a q, 5 \%)$ & $\mathrm{Xe}$ & 300 & - & 3133 & - & - & - & - $\quad[359]$ \\
\hline $\mathrm{Pt}(5 \%) / \mathrm{CdS}$ & $\operatorname{sucrose}(a q, 0.17 \%)^{[h]}$ & $\mathrm{Xe}$ & 500 & r.t. & 830 & - & - & - & $-\quad[214]$ \\
\hline $\mathrm{Pt}(0.5 \%) /{ }^{\mathrm{P} 25} \mathrm{TiO}_{2}$ & lactose $(a q, 1 \%)$ & $\mathrm{Xe}$ (solar) & 300 & 40 & 3000 & - & - & - & - [317] \\
\hline $\mathrm{Pt}(0.5 \%) / \mathrm{TiO}_{2}$ & lactose $(a q, 1 \%)$ & solar & 450 & 40 & 2925 & - & - & - & $-\quad[9]$ \\
\hline $\mathrm{Pt}(0.5 \%))^{/ 25} \mathrm{TiO}_{2}$ & maltose $(a q, 1 \%)$ & $\mathrm{Xe}$ (solar) & 300 & 40 & 2625 & - & - & - & $-\quad[317]$ \\
\hline $\mathrm{Pt}(0.5 \%))^{\mathrm{P25}} \mathrm{TiO}_{2}$ & cellobiose $(a q, 0.29 \mathrm{M})$ & $\mathrm{Xe}$ (solar) & 300 & 40 & 3375 & - & - & - & - $\quad[317]$ \\
\hline $\mathrm{Pt}(0.5 \%))^{\mathrm{P25}} \mathrm{TiO}_{2}$ & cellobiose $(a q, 1 \% \mathrm{w} / \mathrm{v})$ & solar & 450 & 40 & 2775 & - & - & - & $-\quad[9]$ \\
\hline $\mathrm{Pt}(1 \%) /\left(\mathrm{CNT}+\mathrm{TiO}_{2}\right)$ & cellobiose $(a q, 0.02 \mathrm{M})$ & $\mathrm{Hg}$ & 150 & 25 & 79 & - & - & - & $-\quad[159 a]$ \\
\hline $\mathrm{Pt}(1 \%) / \mathrm{TiO}_{2}$ & $\operatorname{starch}(a q, 0.025 \%)^{[i]}$ & $\mathrm{Hg}$ & 125 & 30 & 3750 & - & - & - & $-\quad[62 a]$ \\
\hline $\mathrm{Pt}(1 \%) / \mathrm{TiO}_{2}$ & $\operatorname{starch}(a q, 0.025 \%)$ & $\mathrm{Hg}$ & 125 & 30 & 700 & - & - & - & $-\quad[62 a]$ \\
\hline $\mathrm{Pt}(0.5 \%))^{/ 25} \mathrm{TiO}_{2}$ & $\operatorname{starch}(a q, 1 \%)$ & $\mathrm{Xe}$ (solar) & 450 & 40 & 975 & - & - & - & - $\quad[9]$ \\
\hline $\mathrm{Pt}(5 \%) / \mathrm{TiO}_{2}$ & $\operatorname{starch}(a q, 1,7 \%)$ & $\mathrm{Xe}$ & 500 & r.t. & 80 & - & - & - & $-\quad[108 a]$ \\
\hline $\mathrm{RuO}_{2} / \mathrm{Pt} / \mathrm{TiO}_{2}$ & $\operatorname{starch}\left(a q, 3 \mathrm{~g} \mathrm{~L}^{-1}\right)$ & $\mathrm{Xe}$ & 500 & - & 34 & 16 & - & - & $-\quad[36]$ \\
\hline $\mathrm{Pt}(0.5 \%))^{225} \mathrm{TiO}_{2}$ & cellulose $(a q, 1 \%)^{[j]}$ & $\mathrm{Xe}$ (solar) & 300 & 40 & 900 & - & - & - & - $\quad[317]$ \\
\hline $\mathrm{Pt}(0.5 \%))^{225} \mathrm{TiO}_{2}$ & cellulose $(a q, 1 \%)$ & $\mathrm{Xe}$ (solar) & 450 & 40 & 600 & - & - & - & - $\quad[9]$ \\
\hline $\mathrm{Pt}(0.32 \%) / /^{\mathrm{P} 25} \mathrm{TiO}_{2}$ & cellulose $(a q, 0.67 \%)$ & UV $366 \mathrm{~nm}$ & $15 \times 4$ & - & 225 & - & - & - & - $\quad[313 b]$ \\
\hline $\mathrm{Pt}(0.32 \%) / /^{\mathrm{P} 25} \mathrm{TiO}_{2}$ & cellulose $(a q, 0.67 \%)$ & natural sunlight & $(450)$ & - & 196 & - & - & - & $-\quad[313 b]$ \\
\hline $\mathrm{Pt}(5 \%) / \mathrm{TiO}_{2}$ & cellulose $(a q, 1.7 \%)$ & $\mathrm{Xe}$ & 500 & r.t. & 13 & - & - & - & $-\quad[108 a]$ \\
\hline $\mathrm{RuO}_{2} / \mathrm{Pt} / \mathrm{TiO}_{2}$ & cellulose $\left(a q, 3 \mathrm{~g} \mathrm{~L}^{-1}\right)$ & $\mathrm{Xe}$ & 500 & - & 12 & 7 & - & - & $-\quad[36]$ \\
\hline
\end{tabular}


Table 8. Selected Photocatalytic $\mathrm{H}_{2}$ Production Data from either Raw Biomass or Waste Streams

\begin{tabular}{|c|c|c|c|c|c|c|c|c|}
\hline \multirow[b]{2}{*}{ photocatalyst } & \multirow[b]{2}{*}{ reaction medium $^{[a]}$} & \multirow[b]{2}{*}{ light source } & \multirow{2}{*}{$\begin{array}{l}P / \mathrm{W} \\
\left(I / \mathrm{mW} \mathrm{cm}^{-2}\right)\end{array}$} & \multirow[b]{2}{*}{$T /{ }^{\circ} \mathrm{C}$} & \multicolumn{2}{|c|}{ production rates $/ \mu \mathrm{mol} \mathrm{g} \mathrm{cat}^{-1} \mathrm{~h}^{-1}$} & \multirow[b]{2}{*}{$\Phi_{\mathrm{a}} / \%$} & \multirow[b]{2}{*}{ reference } \\
\hline & & & & & $\mathrm{H}_{2}$ & others & & \\
\hline $\mathrm{Pt}(0.32 \%))^{\mathrm{P} 25} \mathrm{TiO}_{2}$ & $\mathrm{H}_{2} \mathrm{O} /$ rice husks $(l / s, 0.67 \%)$ & UVA (366 nm) & $15 \times 4$ & - & 100 & - & - & {$[313 b]$} \\
\hline $\mathrm{Pt}(0.32 \%))^{\mathrm{P} 25} \mathrm{TiO}_{2}$ & $\mathrm{H}_{2} \mathrm{O} /$ rice husks $(l / s, 0.67 \%)$ & natural sunlight & (45) & - & 95 & - & - & {$[313 b]$} \\
\hline $\mathrm{Pt}(0.32 \%) /^{\mathrm{P} 25} \mathrm{TiO}_{2}$ & $\mathrm{H}_{2} \mathrm{O} /$ alfalfa stems $(l / s, 0.67 \%)$ & UVA (366 nm) & $15 \times 4$ & - & 100 & - & - & {$[313 b]$} \\
\hline $\mathrm{Pt}(5 \%) / \mathrm{TiO}_{2}$ & $\mathrm{H}_{2} \mathrm{O} /$ chlorella $(l / s, 0.3 \%)$ & $\mathrm{Xe}$ & 500 & r.t. & 24 & - & - & [108a] \\
\hline $\mathrm{Pt}(5 \%) / \mathrm{TiO}_{2}$ & $\mathrm{H}_{2} \mathrm{O} /$ chlorella $(l / s, 0.3 \%)^{[b]}$ & $\mathrm{Xe}$ & 500 & r.t. & 90 & - & - & [108a] \\
\hline $\mathrm{Pt}(5 \%) / \mathrm{TiO}_{2}$ & $\mathrm{H}_{2} \mathrm{O} /$ laver $(l / s, 0.3 \%)^{[b]}$ & $\mathrm{Xe}$ & 500 & r.t. & 111 & - & - & [108a] \\
\hline $\mathrm{Pt}(5 \%) / \mathrm{TiO}_{2}$ & $\mathrm{H}_{2} \mathrm{O} /$ olive oil $(l / l, 1.7 \%)$ & $\mathrm{Xe}$ & 500 & r.t. & 11 & - & - & {$[108 \mathrm{a}]$} \\
\hline $\mathrm{Pt}(5 \%) / \mathrm{TiO}_{2}$ & $\mathrm{H}_{2} \mathrm{O} / \operatorname{turf}(l / s, 0.3 \%)$ & $\mathrm{Xe}$ & 500 & r.t. & 7 & - & - & [108a] \\
\hline $\mathrm{Pt}(5 \%) / \mathrm{TiO}_{2}$ & $\mathrm{H}_{2} \mathrm{O} /$ seaweed $(l / s, 0.3 \%)$ & $\mathrm{Xe}$ & 500 & r.t. & 25 & - & - & [214] \\
\hline $\mathrm{Pt}(5 \%) / \mathrm{TiO}_{2}$ & $\mathrm{H}_{2} \mathrm{O} /$ sweet potato $(l / s, 0.3 \%)$ & $\mathrm{Xe}$ & 500 & r.t. & 13 & - & - & [214] \\
\hline $\mathrm{Pt}(0.2 \%) / \mathrm{TiO}_{2}$ & $\mathrm{H}_{2} \mathrm{O} /$ sodium humate $\left(/ / s, 0.1 \mathrm{~g} \mathrm{~L}^{-1}\right)$ & solar simulator & (100) & - & 61 & {$[c]$} & - & [362] \\
\hline $\mathrm{Pt}(0.5 \%) /{ }^{\mathrm{P2} 25} \mathrm{TiO}_{2}$ & $\mathrm{H}_{2} \mathrm{O} /$ olive mill wastewater $(l, 3.3 \%)$ & UVA (366 nm) & $15 \times 4$ & - & 183 & - & - & [321] \\
\hline $\mathrm{Pt}(0.5 \%) /{ }^{\mathrm{P} 25} \mathrm{TiO}_{2}$ & $\mathrm{H}_{2} \mathrm{O} /$ olive mill wastewater $(l, 3.3 \%)^{[d]}$ & UVA $(366 \mathrm{~nm})$ & $15 \times 4$ & - & 342 & - & - & [321] \\
\hline $\mathrm{Pt}(0.5 \%) /{ }^{\mathrm{P} 25} \mathrm{TiO}_{2}$ & $\mathrm{H}_{2} \mathrm{O} /$ olive mill wastewater $(l, 3.3 \%)$ & solar simulator & $(25)$ & - & 20 & - & - & [321] \\
\hline $\mathrm{Au}(0.5 \% \mathrm{~mol}) / \mathrm{TiO}_{2}$ & municipal wastewater ${ }^{[e]}$ & sunlight & - & - & 22 & - & - & [335] \\
\hline $\mathrm{Pt}(0.3 \%) /{ }^{\mathrm{P} 25} \mathrm{TiO}_{2}$ & $\mathrm{H}_{2} \mathrm{O} /$ swine sewage $(1 \%)^{[f]}$ & UVA (366 nm) & $15 \times 2$ & - & 147 & - & 0.5 & [325] \\
\hline $\mathrm{Pt}(0.3 \%))^{\mathrm{P25}} \mathrm{TiO}_{2}$ & $\mathrm{H}_{2} \mathrm{O} /$ swine sewage $(1 \%)^{[f]}$ & solar simulator & (25) & - & 197 & - & - & [325] \\
\hline
\end{tabular}

${ }^{[a]}$ Percentages are given as weight-to-volume unless otherwise stated; reaction media are assumed to be in the form of suspensions. ${ }^{[b]}$ The solution also contained $\mathrm{NaOH}(5 \mathrm{~N}) .[c] \mathrm{Methane}$, ethylene, ethane and oxygen formed at $3,3,1$ and $16 \mu \mathrm{mol} \mathrm{g}_{\mathrm{cat}}{ }^{-1} \mathrm{~h}^{-1}$, respectively. ${ }^{[d]}$ Degreased on magnesium silicate. [e] The ionic conductivity of the aqueous phase was $2.0 \mathrm{mS} \mathrm{cm}{ }^{-1}$

[f] Performed using tap water and at $\mathrm{pH}=10$ 


\section{Conclusions and prospects}

The production of hydrogen from renewable biomass derivatives and water using appropriate photocatalytic materials represent paradigms of highly efficient light harvesting and chemical energy transformation, as illustrated by the plethora of successful examples reported during the last decades. Oxygenated substrates such as alcohols, aldehydes, carboxylic acids or saccharides may undergo selective light-induced reforming (photoreforming) into $\mathrm{H}_{2}$ and $\mathrm{CO}_{2}$ under ambient conditions, as opposed to thermal processes, which result in degradation issues and formation of side products. Photoreforming processes take place by utilising large proportions of the total amount of absorbed photons, or in other words, at elevated quantum yields - apparent quantum efficiencies approach $80 \%$. Moreover, the generation of nearly maximum amounts of $\mathrm{H}_{2}$ relative to those expected from stoichiometry can be attained. This implies that the generated hydrogen carries with it essentially the entire energy content of the initial organic feedstock. In terms of free energy and calorific values, net storage of radiative energy into the formed hydrogen is possible.

The most active materials for the photoreforming of a range of biomass-derived substrates are generally nanocomposites of titanium dioxide and supported metal co-catalysts which display high $\mathrm{H}_{2}$ evolution activity. Since $\mathrm{TiO}_{2}$ is only responsive to UV irradiation, other semiconductors extending the operational spectrum to the visible region are intensely sought. Among the latter, metal sulfides (especially CdS) are widely employed, yet prone to degrade by photocorrosion. Novel alternatives to this include carbon-based materials such as graphitic carbon nitride, graphene oxide or semiconducting polymers. Regarding co-catalysts, noble metals in the form of supported nanoparticles are the best performers, although researchers are boldly turning their eyes to earthabundant metals (chiefly copper and nickel) given their lower cost and safer supply.

Model oxygenated substrates derived from biomass (bio-ethanol or crude glycerol) produce $\mathrm{H}_{2}$ at extremely high rates by photocatalysis, and therefore, they can be considered "hydrogen carriers". Integration of their photoreforming into power generation systems might be envisaged. More complex and structurally intricate biomass derivatives tend to result in slower $\mathrm{H}_{2}$ production rates, and thus, their use is expected to become feasible in cases where they are part of low-cost byproducts or waste to be valorised. From this perspective, agricultural residues rich in lignocelluloses and wastewater streams containing significant concentration of degradable organic matter might become valuable resources for the generation of hydrogen. In general, any biomass photoreforming strategy entails an additional benefit regarding sustainability, since it can be activated by the essentially inextinguishable solar light as the only energy input. 


\section{Acknowledgements}

Financial support from the Spanish Government-MINECO through "Severo Ochoa" (SEV 20120267) is acknowledged. The European Union is also acknowledged by the SynCatMatch project (ERC-AdG-2014-671093). 
Figure 1. Schematic representation of water splitting, biomass $\left(\mathrm{C}_{x} \mathrm{H}_{y} \mathrm{O}_{z}\right)$ oxidation and biomass reforming reactions over an irradiated $\mathrm{M} / \mathrm{TiO}_{2}(\mathrm{M}=$ noble metal $)$ photocatalyst. All reactions are initiated by light absorption on $\mathrm{M} / \mathrm{TiO}_{2}$ generating separated charges $\left(\mathrm{e}^{-}\right.$and $\left.\mathrm{h}^{+}\right)$in the conduction (CB) and valence bands (VB), respectively. In anaerobic conditions, production of $\mathrm{H}_{2}(i)$ and $\mathrm{O}_{2}$ (iii), that is, water splitting, can take place. Oxidation of oxygenated organic compounds ( $\mathrm{iv}$ ) proceeds in the presence of $\mathrm{O}_{2}$, which is then reduced to water (ii). Biomass photoreforming takes place in the absence of $\mathrm{O}_{2}$, and can be thus considered a combination of water splitting and biomass oxidation. Adapted from reference [9].

Figure 2. Typical routes for the production of $\mathrm{H}_{2}$ from lignocellulosic biomass, including (i) direct gasification pathways and (ii) indirect pathways based on the reforming of oxygenated derivatives.

Figure 3. Biomass-derived oxygenated substrates, classified into generic families (in italics) according to their chemical structures and functionalities. The substances are related to their sources and the corresponding transformation processes involved: $i$ : pyrolysis; ii: hydrolysis; iii: transesterification; $i v$ : hydrogenation; $v$ : dehydration; $v i$ : fermentation. The most frequently studied oxygenates for the production of $\mathrm{H}_{2}$ via photocatalytic reforming are highlighted in bold font.

Figure 4. Free energy change $v s$. temperature for the gas phase reforming reaction of different substrates in the presence of water, producing $\mathrm{CO}_{2}$ and $\mathrm{H}_{2}$; methane (solid line), ethanol (dotted line) and methanol (dashed line). Data have been calculated considering the following conditions: $P($ substrate $)=2 \mathrm{~atm} ; \mathrm{eq}\left(\mathrm{H}_{2} \mathrm{O}\right) / \mathrm{eq}($ substrate $)=2$; conversion $=0.5 \%$ molar $(\mathrm{C})$ yield .

Figure 5. Energy profiles of ethanol reforming (centre), ethanol combustion (left) and $\mathrm{H}_{2}$ combustion (right). Standard free energy and enthalpy changes, normalised for one mole of ethanol, are represented along the schematic representation of each transformation.

Figure 6. Heats of combustion, normalised per mole of carbon, of several model oxygenates (grey bars) and of the equivalent amounts of $\mathrm{H}_{2}$ (white bars) which can be obtained from them by reforming (values in $\mathrm{kJ} \mathrm{mol}^{-1}$ indicated inside the bars). The relative increase in heating values is indicated above the $\mathrm{H}_{2}$ bars.

Figure 7. Left: Transient spectra of $\mathrm{TiO}_{2}$ irradiated by a $355 \mathrm{~nm}$ laser pulse at different delay times. Right: Possible transitions in $\mathrm{TiO}_{2}$ upon absorption of photons of energy higher than its band gap and subsequent absorption of the photo-excited charges upon further IR absorption: intra-band electron transitions (a), trapped electron transition to the conduction band (b), intraband hole transition (c), and trapped hole transition to the valence band. According to energy differences, it 
was suggested that electron transitions are responsible for the feature-less absorbance in the IR spectrum. Left: Reprinted with permission from reference [46]. Copyright (2001) American Chemical Society. Right: Reprinted with permission from reference [45a]. Copyright (2001) Elsevier.

Figure 8. Left: Transient IR $\left(2000 \mathrm{~cm}^{-1}\right)$ absorption observed on $\mathrm{TiO}_{2}$ irradiated by a $355 \mathrm{~nm}$ laser pulse at $t=0$ in the presence or absence of $\mathrm{O}_{2}(10$ Torr $)$. Right: Transient IR $\left(2000 \mathrm{~cm}^{-1}\right)$ absorption observed on $\mathrm{TiO}_{2}$ irradiated by a $355 \mathrm{~nm}$ laser pulse at $t=0$ in the presence or absence of $\mathrm{H}_{2} \mathrm{O}$ (10 Torr). Reprinted with permission from reference [46]. Copyright (2001) American Chemical Society.

Figure 9. Left: Transient IR $\left(2000 \mathrm{~cm}^{-1}\right)$ absorption observed on $\mathrm{TiO}_{2}$ and $\mathrm{Pt} / \mathrm{TiO}_{2}$ irradiated by a $355 \mathrm{~nm}$ laser pulse under vacuum at $t=0$ in the presence or absence of $\mathrm{O}_{2}$ (10 Torr). Centre: Transient IR $\left(2000 \mathrm{~cm}^{-1}\right)$ absorption observed on $\mathrm{Pt} / \mathrm{TiO}_{2}$ irradiated by a $355 \mathrm{~nm}$ laser pulse at $t=0$ in the presence or absence of $\mathrm{H}_{2} \mathrm{O}$ (10 Torr). Right: Transient IR $\left(2000 \mathrm{~cm}^{-1}\right)$ absorption observed on $\mathrm{Pt} / \mathrm{TiO}_{2}$ irradiated by a $355 \mathrm{~nm}$ laser pulse at $t=0$ in the presence of $\mathrm{H}_{2} \mathrm{O} / \mathrm{CH}_{3} \mathrm{OH}$ mixtures (10 Torr). Left and centre: Reprinted with permission from reference [46]. Copyright (2001) American Chemical Society. Right: Reprinted with permission from reference [52]. Copyright (2003) American Chemical Society.

Figure 10. UV-visible absorption spectra of $\mathrm{TiO}_{2}$ nanocrystalline films in the ground state and of trapped holes after excitation by a $355 \mathrm{~nm}$ laser (A), and time profiles of transient absorption $(400 \mathrm{~nm})$ of $\mathrm{TiO}_{2}$ in the presence of air, water or different alcohols (B). The ultra-fast hole consumption by alcohol (photo-)oxidation is evidenced by the decay of the transient hole signal at times shorter than $1 \mathrm{~ns}$. Reprinted with permission from reference [56]. Copyright (2006) American Chemical Society.

Figure 11. Initial rate of $\mathrm{H}_{2}$ evolution ( $r$ ) from aqueous suspensions of $\mathrm{Pt} / \mathrm{TiO}_{2}$ versus the initial concentration of glucose $(\mathrm{C})$. The inset shows the linear relationship $(1 / r v s .1 / C)$ based on the Langmuir-Hinshelwood kinetic model. Reprinted with permission from reference [62a]. Copyright (2008) Elsevier.

Figure 12. Suggested reactions occurring on photo-activated $\mathrm{TiO}_{2}$ under aerobic (top, adapted with permission from reference [66a]; copyright (1995) American Chemical Society) or anaerobic (bottom) conditions, resulting in overall oxidation or reforming processes, respectively. Both pathways involve oxidation of organic substances $(\{$ red $\})$ into primary products $(\{\mathrm{ox}\})$, either directly for adsorbates (ad) or indirectly by hydroxyl radicals for loosely adsorbed or solvated species (aq). In aerobic oxidation, $\mathrm{O}_{2}$ is expected to be transformed into a number of activated oxygen species (highlighted in bold), which may be responsible in a substantial part for the complete mineralisation of organic matter in thermal reactions (elementary steps illustrated in grey). In contrast, in the absence of $\mathrm{O}_{2}$, all electron transfer reactions are expected to be activated by light, and primarily oxidised species should become the reduced substrates for further oxidation steps (ideally until complete mineralisation), as indicated by dashed arrows. 
Figure 13. Schematic illustration of $\mathrm{TiO}_{2}$ surfaces in the presence of water (a), and after ethanol physisorption in mobile water layers (b); the latter scenario evolved either to water removal and ethanol condensation with titanol residues to form adsorbed ethoxide upon increasing temperature (c), or to ethanol mineralisation under aerobic conditions and UV irradiation. Reprinted with permission from reference [78]. Copyright (2003) American Chemical Society.

Figure 14. Schematic representation of the adsorption of molecules containing carboxylate functionalities on different semiconductor surfaces. Top: adsorption of formate and oxalate on $\mathrm{TiO}_{2}$ via carboxylate or geminal "dioxo" modes, respectively. Bottom: adsorption of lactic acid on $\mathrm{TiO}_{2}$ or CdS surfaces via strong "dioxo" bonding or electrostatic carboxylate interaction, respectively. Top: Reprinted with permission from reference [81]. Copyright (2003) Elsevier. Bottom: Reprinted with permission from reference [83]. Copyright (1998) The Chemical Society of Japan.

Figure 15. Schematic representation of the proposed $\mathrm{CO}$ mechanistic route for the photoreforming of an alcohol on a $\mathrm{Pd} / \mathrm{TiO}_{2}$ material, whereby the substrate is dissociated on the metallic particle into adsorbed $\mathrm{CO}$, adsorbed atomic hydrogen, and an alkane. Hydrogen atoms would couple to form gaseous $\mathrm{H}_{2}$. Upon light absorption, the generated holes and electrons (represented by the $\mathrm{O}^{-} / \mathrm{Ti}^{3+}$ pair) would unleash $\mathrm{CO}$ oxidation into $\mathrm{CO}_{2}$, and water reduction into $\mathrm{H}_{2}$, respectively. Reprinted with permission from reference [90b]. Copyright (2011) Elsevier.

Figure 16. Schematic representation of the proposed carbonyl mechanism for the photoreforming of an alcohol, whereby the adsorbed alkoxide is dehydrogenated by a hole at the surface of $\mathrm{TiO}_{2}$ (a), and a subsequent electron transfer results in the corresponding ketone or aldehyde (b). Further oxidative C-C scission would lead to a carboxylic acid and an alkyl radical ( $\mathrm{R}^{\circ}$ or $\mathrm{R}^{\prime \prime}$, (c)). The acid would then decarboxylate into $\mathrm{CO}_{2}$ and alkane, whereas alkyl radicals may be either oxidised by $[\mathrm{OH}]^{\cdot}$ into alcohols or coupled to $\mathrm{H}^{*}$ or other alkyl radicals to form $\mathrm{RH}$ or $\mathrm{R}_{2}$-type alkanes (d). Finally, protons formally released from the different reaction steps would be reduced with concomitant $\mathrm{H}_{2}$ evolution. Reprinted with permission from reference [96]. Copyright (2011) Elsevier.

Figure 17. Suggested acetic acid photoreforming reaction network, illustrating the varied transformations which can take place from the methyl radical formed by the initial decarboxylation. Reprinted with permission from reference [37]. Copyright (1984) American Chemical Society.

Figure 18. Suggested and consolidated mechanism for the reforming of oxygenated substrates (R, $\mathrm{R}^{\prime}=\mathrm{H}$ or $\mathrm{C}_{n} ; \mathrm{C}_{n}=$ linear backbone having $n$ carbon atoms), promoted by photo-generated holes $\left(\mathrm{h}^{+}\right)$ and electrons $\left(\mathrm{e}^{-}\right)$. On the oxidation side, the "helical", pseudo-cyclic route proposed accounts for the sequential transformation of primary and secondary alcohols into the corresponding carbonyl compounds (aldehydes and ketones, respectively), the oxidative dissociation of the latter into carboxylic acids and alkyl radicals, and the oxidative decarboxylation of the acids. At the end of each cycle the alkyl radicals would be coupled to hydroxyl radicals forming new, shorter-chain 
alcohols ( $\mathrm{R}$ or R' become $\mathrm{C}_{n-1}$ chains), thus closing the helical loop (dashed arrows). Reforming of the smaller alcohols would continue, ideally until complete oxidation into $\mathrm{CO}_{2}$. Molecules with multiple hydroxyl groups, such as polyols or saccharides would be efficiently oxidised via the inner cycle. Protons would be reduced to hydrogen atoms and subsequently evolve as $\mathrm{H}_{2}$ (left), whereas radical coupling side reactions would lead to alkane by-products. All depicted species have been observed experimentally except those shown as shaded structures. Any of the oxidation steps depicted can in theory be promoted by holes or by $[\mathrm{OH}]^{*}$, and the formal representation in either way is only for illustration purposes. Likewise, the involvement of protons is also proposed from a formal point of view, and the real situation is expected to depend on the $\mathrm{pH}$ of the solution.

Figure 19. Energy positions of conduction band edges and valence band edges for selected metal oxide (top) and metal sulfide (middle) semiconductors. Horizontal lines represent the redox potentials for water reduction and oxidation. A comparison of some metal oxides or sulfides to counterparts composed solely by non-metals is also depicted (bottom). Top and middle: Reprinted with permission from reference [41]. Copyright (2000) Mineralogical Society of America. Bottom: Reprinted with permission from reference [5d]. Copyright (2009) The Royal Society of Chemistry.

Figure 20. Illustration showing the dependence of $\mathrm{TiO}_{2}$ phases, surface area and crystallinity as a function of calcination temperature. This simplistic (yet concise) representation shows that increasing temperatures result in higher mobility of carriers and diminished amount of surface active sites, in principle enhancing and impairing photocatalytic activities, respectively. Reprinted with permission from reference [5d]. Copyright (2009) The Royal Society of Chemistry.

Figure 21. Energy diagram of rutile/anatase junctions based on XPS data. $E^{\mathrm{CBM}}$ and $E^{\mathrm{VBM}}$ denote the positions of the conduction band and valence band, respectively. $\Delta E_{\mathrm{C}}$ and $\Delta E_{\mathrm{V}}$ (valence band offset and conduction band offset, respectively) dictate deeper energy states for anatase, and thus, preferential transfer of photo-generated electron from rutile to anatase. $\Delta E_{\mathrm{CL}}$ is the core level offset between the Ti $2 p_{3 / 2}$ core levels. Reprinted with permission from reference [151]. Copyright (2013) Macmillan Publishers Limited.

Figure 22. Calculated density of states of non-metal-doped $\mathrm{TiO}_{2}$. The doping elements were located at substitutional sites for $\mathrm{O}$ atoms, except in cases where $\mathrm{N}$ was located only at interstitial sites $\left(\mathrm{N}_{\mathrm{i}^{-}}\right.$ doped structures) or at both interstitial and substitutional sites ( $\mathrm{N}_{\mathrm{i}+\mathrm{s}}$-doped structures). Substitutional nitrogen doping was shown to add new states above the valence band, resulting in effective band gap narrowing. Reprinted with permission from reference [169]. Copyright (2001) American Association for the Advancement of Science. 
Figure 23. The idealised model of the growth of metallic nanoparticles on a surface. As the diameter of the particles increases, the perimeter length reaches a maximum, to decrease again above that value. Reprinted with permission from reference [91a]. Copyright (2003) Elsevier.

Figure 24. A typical TEM micrograph of a $\mathrm{Pt} / \mathrm{TiO}_{2}$ material prepared by deposition-precipitation of platinum ( $2 \%$ by weight) on a P25 powder. Highly dispersed Pt nanoparticles can be seen on the surface of larger titania particles. Reprinted with permission from reference [266]. Copyright (2013) Springer.

Figure 25. High resolution TEM micrographs of random $\mathrm{AuPd}$ alloy (a), $\mathrm{Au}_{\text {shell }}-\mathrm{Pd}_{\text {core }}$ (b) and $\mathrm{Pd}_{\text {shell }}-\mathrm{Au}_{\text {core }}(\mathrm{c})$ nanoparticles. Reprinted with permission from reference [287]. Copyright (2014) American Chemical Society.

Figure 26. HRTEM micrographs of $\mathrm{MoS}_{2}(1 \%) / \mathrm{CdS}$ prepared by impregnation of molybdenum on the support at two different magnifications. The image on the right shows the intimate contact between both sulfide phases which presumably facilitates electron transfer across the junction. Reprinted with permission from reference [291b]. Copyright (2008) American Chemical Society.

Figure 27. Structures of two hydrogen-evolving Co complexes used in conjunction with CdTe quantum dots. Reprinted with permission from reference [239a]. Copyright (2014) American Chemical Society.

Figure 28. Emission spectra of a Xe lamp with no filters applied (grey) and a solar simulator, equipped with a Xe lamp and a standard AM 1.5 filter (black).

Figure 29. Arrhenius plots for acetone formation in irradiated $(366 \mathrm{~nm})$ 2-propanol suspensions containing bare $\mathrm{TiO}_{2}$ (anatase, open circles) or $\mathrm{Pt}(0.5 \%) / \mathrm{TiO}_{2}$ (filled circles) at different radiation intensities: $3.2 \times 10^{-7}$ (i), $0.65 \times 10^{-7}$ (ii), $0.14 \times 10^{-7}$ mol(photons) $\mathrm{s}^{-1}$ (iii). The flat areas $\left(E_{\mathrm{a}}=0\right)$ at high temperatures represent regimes where reaction rates are only limited by irradiance, whereas classical Arrhenius behaviour is observed at lower temperatures (sloped areas, $E_{\mathrm{a}}>0$ ). Reprinted with permission from reference [70]. Copyright (1987) The Royal Society of Chemistry.

Figure 30. Energy diagram showing the Nernstian behaviour of the redox potentials of water reduction and oxidation, hydroxide oxidation and the acetaldehyde/ethanol pair, as compared to the less $\mathrm{pH}$-dependent band positions of CdS. As a result of this situation, it was claimed that direct 
oxidation of ethanol by photo-generated holes prevails in acidic and neutral media, whereas a twostep mechanism via $[\mathrm{OH}]^{\bullet}$ is suggested to take place above a $\mathrm{pH}$ crossover value. Reprinted with permission from reference [319]. Copyright (2014) Macmillan Publishers Limited.

Figure 31. Dependence of the rates of production of $\mathrm{H}_{2}$ and $\mathrm{CH}_{4}$ on $\mathrm{pH}$ from irradiated (Xe lamp, $500 \mathrm{~W}$ ) suspensions of $\mathrm{Pt} / \mathrm{TiO}_{2}$ (anatase) in $\mathrm{H}_{2} \mathrm{O} / \mathrm{CH}_{3} \mathrm{COOH}(6: 1 \mathrm{v} / \mathrm{v})$. It is worth noting the drastic decrease in selectivity towards methane at high $\mathrm{pH}$. Reprinted with permission from reference [216]. Copyright (1985) Elsevier.

Figure 32. Variation of the maximum $\mathrm{H}_{2}$ production rates as a function of the volume fraction of ethanol from irradiated (black light, $0.80 \mathrm{~mW} \mathrm{~cm}^{-2}$ ) $\mathrm{Pt} / \mathrm{TiO}_{2}$ suspensions in aqueous media. Reprinted with permission from reference [98]. Copyright (2007) Elsevier.

Figure 33. Proposed reaction sequence for methanol oxidation during photoreforming on $\mathrm{M} / \mathrm{TiO}_{2}$ ( $\mathrm{M}=$ noble metal), showing the step-wise charge-transfer transformation and the adsorptiondesorption events. Reprinted with permission from reference [77]. Copyright (2011) Elsevier.

Figure 34. Production rates of $\mathrm{H}_{2}, \mathrm{CO}_{2}$ and acetaldehyde from the reaction of gaseous ethanol/water mixtures on $\mathrm{M} / \mathrm{TiO}_{2}\left(\mathrm{M}=\mathrm{Au}\right.$ or $\mathrm{Au}_{3} \mathrm{Cu}$ nanoalloy). Dehydrogenation is favoured at high ethanol fractions, whereas photoreforming also takes place under water-rich regimes. Reprinted with permission from reference [288]. Copyright (2015) Springer.

Figure 35. Time profiles of the production rates of $\mathrm{H}_{2}$ (trace $\mathrm{b}$ ) and $\mathrm{CO}_{2}$ (trace $\mathrm{c}$ ) from irradiated (simulated sunlight) aqueous $\mathrm{Pt} / \mathrm{TiO}_{2}$ suspensions containing variable ethanol concentrations: $0.21 \mathrm{mM}(\mathrm{A}), 0.43 \mathrm{mM}(\mathrm{B})$, and $0.86 \mathrm{mM}(\mathrm{C})$ under Ar flow $\left(20 \mathrm{~cm}^{3} \mathrm{~min}^{-1}\right)$; maximum rates can be observed at shorter irradiation times for $\mathrm{H}_{2}$ than for $\mathrm{CO}_{2}$. Hydrogen production rates from pure water (trace a) are shown for comparison. Reprinted with permission from reference [38a]. Copyright (2007) Elsevier.

Figure 36. Scheme of the oxidative coupling of ethanol to yield 2,3-butanediol suggested the transient formation of 1-hydroxyethyl radicals on irradiated photocatalysts under particular conditions, as reported in references [97a] and [231a]. 
Figure 37. Reaction sequence of glycerol photoreforming, based on results using titania-based materials, as suggested in references [272c] and [337]. Reprinted with permission from reference [337]. Copyright (2013) The Royal Society of Chemistry.

Figure 38. Time profiles of $\mathrm{H}_{2}$ production rates from irradiated (simulated sunlight) aqueous $\mathrm{Pt} / \mathrm{TiO}_{2}$ suspensions containing different oxygenated substrates: formic acid, acetic acid and acetaldehyde (concentrations as indicated in the graph) under Ar flow $\left(20 \mathrm{~cm}^{3} \mathrm{~min}^{-1}\right)$. Hydrogen production rates from pure water (trace d) are shown for comparison. Reprinted with permission from reference [38a]. Copyright (2007) Elsevier.

Figure 39. Reaction sequence of possible primary lactic acid reactions upon irradiation. The decarboxylation route ( $i$ ) may lead to acetaldehyde and ethanol, whereas the dehydrogenation route (ii) yields pyruvic acid as the main product in the first step, although the latter may in turn evolve by decarboxylation to acetic acid. In all cases, the formed products are expected to undergo further photoreforming under. 


\section{References}

[1] (a) G.W. Huber, S. Iborra, A. Corma, Chem. Rev., 106 (2006) 4044-4098; (b) G.W. Huber, A. Corma, Angew. Chem., Int. Ed., 46 (2007) 7184-7201; (c) M.J. Climent, A. Corma, S. Iborra, Green Chem., 16 (2014) 516-547; (d) D.M. Alonso, J.Q. Bond, J.A. Dumesic, Green Chem., 12 (2010) 1493-1513; (e) A.J. Ragauskas, C.K. Williams, B.H. Davison, G. Britovsek, J. Cairney, C.A. Eckert, W.J. Frederick, J.P. Hallett, D.J. Leak, C.L. Liotta, J.R. Mielenz, R. Murphy, R. Templer, T. Tschaplinski, Science, 311 (2006) 484-489; (f) D. Tilman, R. Socolow, J.A. Foley, J. Hill, E. Larson, L. Lynd, S. Pacala, J. Reilly, T. Searchinger, C. Somerville, R. Williams, Science, 325 (2009) 270-271; (g) S. Zinoviev, F. Müller-Langer, P. Das, N. Bertero, P. Fornasiero, M. Kaltschmitt, G. Centi, S. Miertus, ChemSusChem, 3 (2010) 1106-1133.

[2] (a) N.S. Lewis, D.G. Nocera, Proc. Natl. Acad. Sci. U. S. A., 103 (2006) 15729-15735; (b) V. Balzani, N. Armaroli, Energy for a Sustainable World: From the Oil Age to a Sun-Powered Future, Wiley, 2010; (c) N. Armaroli, V. Balzani, Angew. Chem., Int. Ed., 46 (2007) 52-66. [3] (a) A. Corma, H. Garcia, J. Catal., 308 (2013) 168-175; (b) S. Bensaid, G. Centi, E. Garrone, S. Perathoner, G. Saracco, ChemSusChem, 5 (2012) 500-521; (c) A.J. Bard, M.A. Fox, Acc. Chem. Res., 28 (1995) 141-145.

[4] (a) J.A. Turner, Science, 305 (2004) 972-974; (b) A. Züttel, A. Borgschulte, L. Schlapbach, in, Wiley, 2011; (c) J. Turner, G. Sverdrup, M.K. Mann, P.-C. Maness, B. Kroposki, M. Ghirardi, R.J. Evans, D. Blake, Int. J. Energy Res., 32 (2008) 379-407; (d) N. Armaroli, V. Balzani, ChemSusChem, 4 (2011) 21-36; (e) G. Centi, R.A. van Santen, in, Wiley, 2008.

[5] (a) S.Y. Reece, J.A. Hamel, K. Sung, T.D. Jarvi, A.J. Esswein, J.J.H. Pijpers, D.G. Nocera, Science, 334 (2011) 645-648; (b) K. Maeda, K. Teramura, D. Lu, T. Takata, N. Saito, Y. Inoue, K. Domen, Nature, 440 (2006) 295; (c) D.G. Nocera, Acc. Chem. Res., 45 (2012) 767-776; (d) A. Kudo, Y. Miseki, Chem. Soc. Rev., 38 (2009) 253-278.

[6] (a) J. Schneider, M. Matsuoka, M. Takeuchi, J. Zhang, Y. Horiuchi, M. Anpo, D.W. Bahnemann, Chem. Rev., 114 (2014) 9919-9986; (b) T.L. Thompson, J.T. Yates, Jr., Chem. Rev., 106 (2006) 4428-4453; (c) B. Ohtani, Phys. Chem. Chem. Phys., 16 (2014) 1788-1797.

[7] (a) Y. Ma, X. Wang, Y. Jia, X. Chen, H. Han, C. Li, Chem. Rev., 114 (2014) 9987-10043; (b) M. Cargnello, A. Gasparotto, V. Gombac, T. Montini, D. Barreca, P. Fornasiero, Eur. J. Inorg. Chem., (2011) 4309-4323; (c) D. Barreca, G. Carraro, V. Gombac, A. Gasparotto, C. Maccato, P. Fornasiero, E. Tondello, Adv. Funct. Mater., 21 (2011) 2611-2623; (d) K. Shimura, H. Yoshida, Energy Environ. Sci., 4 (2011) 2467-2481; (e) X.B. Chen, S.H. Shen, L.J. Guo, S.S. Mao, Chem. Rev., 110 (2010) 6503-6570.

[8] (a) Y.H. Li, J. Xing, Z.J. Chen, Z. Li, F. Tian, L.R. Zheng, H.F. Wang, P. Hu, H.J. Zhao, H.G. Yang, Nat. Commun., 4 (2013) 3500, 3507 pp; (b) S. Sato, J.M. White, Chem. Phys. Lett., 72 (1980) 83-86.

[9] D.I. Kondarides, V.M. Daskalaki, A. Patsoura, X.E. Verykios, Catal. Lett., 122 (2008) 26-

32.

[10] A. Corma, S. Iborra, A. Velty, Chem. Rev., 107 (2007) 2411-2502.

[11] D.L. Klass, Biomass for Renewable Energy, Fuels, and Chemicals, Elsevier Science, San Diego, 1998.

[12] T. Searchinger, R. Heimlich, R.A. Houghton, F.X. Dong, A. Elobeid, J. Fabiosa, S. Tokgoz, D. Hayes, T.H. Yu, Science, 319 (2008) 1238-1240.

[13] G.W. Crabtree, M.S. Dresselhaus, M.V. Buchanan, Phys. Today, 57 (2004) 39-44.

[14] R.M. Navarro, M.A. Peña, J.L.G. Fierro, Chem. Rev., 107 (2007) 3952-3991.

[15] (a) R.M. Navarro, M.C. Sanchez-Sanchez, M.C. Alvarez-Galvan, F. del Valle, J.L.G. Fierro, Energy Environ. Sci., 2 (2009) 35-54; (b) T.A. Milne, C.C. Elam, R.J. Evans, in: Other Information: PBD: 1 Feb 2002, 2002, pp. Medium: ED; Size: 82 pages; Other:

http://www.nrel.gov/docs/legosti/old/36262.pdf. 
[16] A. Demirbaş, Energy Conv. Manag., 42 (2001) 1357-1378.

[17] (a) G.W. Huber, J.A. Dumesic, Catal. Today, 111 (2006) 119-132; (b) T.P. Vispute, G.W. Huber, Green Chem., 11 (2009) 1433-1445; (c) R.R. Davda, J.W. Shabaker, G.W. Huber, R.D. Cortright, J.A. Dumesic, Appl. Catal., B, 56 (2005) 171-186.

[18] (a) D. Wang, S. Czernik, D. Montane, M. Mann, E. Chornet, Ind. Eng. Chem. Res., 36 (1997) 1507-1518; (b) L. Garcia, R. French, S. Czernik, E. Chornet, Appl. Catal., A, 201 (2000) 225-239; (c) C. Rioche, S. Kulkarni, F.C. Meunier, J.P. Breen, R. Burch, Appl. Catal., B, 61 (2005) 130-139.

[19] T. Milne, F. Agblevor, M. Davis, S. Deutch, D. Johnson, A Review of the Chemical Composition of Fast-Pyrolysis Oils from Biomass, in: A.V. Bridgwater, D.G.B. Boocock (Eds.) Developments in Thermochemical Biomass Conversion, Springer Netherlands, 1997, pp. 409-424. [20] J.P. Diebold, in, Thermalchemie, Inc, National Renewable Energy Laboratory, 2000. [21] (a) R.D. Cortright, R.R. Davda, J.A. Dumesic, Nature, 418 (2002) 964-967; (b) G.W. Huber, J.W. Shabaker, J.A. Dumesic, Science, 300 (2003) 2075-2077.

[22] M. Scott, A.M. Nadeem, G.I. Waterhouse, H. Idriss, MRS Online Proc. Libr., 1326 (2011) 98-107.

[23] A.L. Linsebigler, G. Lu, J.T. Yates, Chem. Rev., 95 (1995) 735-758.

[24] V.M. Daskalaki, D.I. Kondarides, Catal. Today, 144 (2009) 75-80.

[25] M.d.O. Melo, L.A. Silva, J. Braz. Chem. Soc., 22 (2011) 1399-1406.

[26] M. Bowker, Green Chem., 13 (2011) 2235-2246.

[27] I. Rossetti, ISRN Chem. Eng., (2012) 964936.

[28] L. Clarizia, D. Spasiano, I. Di Somma, R. Marotta, R. Andreozzi, D.D. Dionysiou, Int. J. Hydrogen Energy, 39 (2014) 16812-16831.

[29] K.A. Connelly, H. Idriss, Green Chem., 14 (2012) 260-280.

[30] (a) P. Lianos, J. Hazard. Mater., 185 (2011) 575-590; (b) N. Ibrahim, S.K. Kamarudin, L.J. Minggu, J. Power Sources, 259 (2014) 33-42.

[31] X. Lang, X. Chen, J. Zhao, Chem. Soc. Rev., 43 (2014) 473-486.

[32] J. Xu, G.F. Froment, AIChE J., 35 (1989) 88-96.

[33] (a) A.N. Fatsikostas, X.E. Verykios, J. Catal., 225 (2004) 439-452; (b) I. Fishtik, A.

Alexander, R. Datta, D. Geana, Int. J. Hydrogen Energy, 25 (1999) 31-45.

[34] (a) A.N. Fatsikostas, D.I. Kondarides, X.E. Verykios, Chem. Commun., (2001) 851-852;

(b) A.N. Fatsikostas, D.I. Kondarides, X.E. Verykios, Catal. Today, 75 (2002) 145-155; (c) D.K. Liguras, D.I. Kondarides, X.E. Verykios, Appl. Catal., B, 43 (2003) 345-354.

[35] (a) E.Y. Garcia, M.A. Laborde, Int. J. Hydrogen Energy, 16 (1991) 307-312; (b) K. Vasudeva, N. Mitra, P. Umasankar, S.C. Dhingra, Int. J. Hydrogen Energy, 21 (1996) 13-18.

[36] T. Kawai, T. Sakata, Nature, 286 (1980) 474-476.

[37] T. Sakata, T. Kawai, K. Hashimoto, J. Phys. Chem., 88 (1984) 2344-2350.

[38] (a) A. Patsoura, D.I. Kondarides, X.E. Verykios, Catal. Today, 124 (2007) 94-102; (b) T.A. Kandiel, I. Ivanova, D.W. Bahnemann, Energy Environ. Sci., 7 (2014) 1420-1425; (c) G.N.

Nomikos, P. Panagiotopoulou, D.I. Kondarides, X.E. Verykios, Appl. Catal., B, 146 (2014) 249257.

[39] (a) T. Shiragami, T. Tomo, T. Matsumoto, M. Yasuda, Bull. Chem. Soc. Jpn., 86 (2013) 382-389; (b) T. Shiragami, T. Tomo, H. Tsumagari, R. Yuki, T. Yamashita, M. Yasuda, Chem. Lett., 41 (2012) 29-31; (c) M. Yasuda, R. Kurogi, H. Tsumagari, T. Shiragami, T. Matsumoto, Energies, 7 (2014) 4087-4097.

[40] D.R. Lide, CRC Handbook of Chemistry and Physics, 84th Edition, Taylor \& Francis, 2003.

[41] Y. Xu, M.A.A. Schoonen, Am. Mineral., 85 (2000) 543-556.

[42] B. Ohtani, Chem. Lett., 37 (2008) 217-229.

[43] P. Pichat, J.M. Herrmann, J. Disdier, H. Courbon, M.N. Mozzanega, Nouv. J. Chim., 5 (1981) 627-636. 
[44] (a) A. Sclafani, L. Palmisano, M. Schiavello, V. Augugliaro, S. Coluccia, L. Marchese, New J. Chem., 12 (1988) 129-135; (b) V. Augugliaro, L. Palmisano, M. Schiavello, A. Sclafani, J. Catal., 99 (1986) 62-71.

[45] (a) A. Yamakata, T. Ishibashi, H. Onishi, Chem. Phys. Lett., 333 (2001) 271-277; (b) A. Yamakata, T. Ishibashi, H. Onishi, J. Mol. Catal. A: Chem., 199 (2003) 85-94.

[46] A. Yamakata, T. Ishibashi, H. Onishi, J. Phys. Chem. B, 105 (2001) 7258-7262.

[47] (a) X. Yang, C. Salzmann, H. Shi, H. Wang, M.L.H. Green, T. Xiao, J. Phys. Chem. A, 112

(2008) 10784-10789; (b) R.F. Howe, M. Gratzel, J. Phys. Chem., 89 (1985) 4495-4499.

[48] B. Ohtani, Y. Ogawa, S. Nishimoto, J. Phys. Chem. B, 101 (1997) 3746-3752.

[49] C. Renz, Helv. Chim. Acta, 4 (1921) 961-968.

[50] A. Yamakata, T. Ishibashi, H. Onishi, Int. J. Photoenergy, 5 (2003) 7-9.

[51] A. Yamakata, T. Ishibashi, H. Onishi, J. Phys. Chem. B, 106 (2002) 9122-9125.

[52] A. Yamakata, T.A. Ishibashi, H. Onishi, J. Phys. Chem. B, 107 (2003) 9820-9823.

[53] A. Yamakata, T. Ishibashi, H. Onishi, Chem. Phys. Lett., 376 (2003) 576-580.

[54] A. Yamakata, T. Ishibashi, H. Kato, A. Kudo, H. Onishi, J. Phys. Chem. B, 107 (2003) $14383-14387$.

[55] I.A. Shkrob, M.C. Sauer, Jr., J. Phys. Chem. B, 108 (2004) 12497-12511.

[56] Y. Tamaki, A. Furube, M. Murai, K. Hara, R. Katoh, M. Tachiya, J. Am. Chem. Soc., 128 (2006) 416-417.

[57] A. Dickinson, D. James, N. Perkins, T. Cassidy, M. Bowker, J. Mol. Catal. A: Chem., 146 (1999) 211-221.

[58] Y. Li, J. Wang, S. Peng, G. Lu, S. Li, Int. J. Hydrogen Energy, 35 (2010) 7116-7126.

[59] S. Nishimoto, B. Ohtani, T. Kagiya, J. Chem. Soc., Faraday Trans. 1, 81 (1985) 2467-2474.

[60] Y.X. Li, G.X. Lu, S.B. Li, Appl. Catal. A-Gen., 214 (2001) 179-185.

[61] X.-J. Zheng, L.-F. Wei, Z.-H. Zhang, Q.-J. Jiang, Y.-J. Wei, B. Xie, M.-B. Wei, Int. J. Hydrogen Energy, 34 (2009) 9033-9041.

[62] (a) X. Fu, J. Long, X. Wang, D.Y.C. Leung, Z. Ding, L. Wu, Z. Zhang, Z. Li, X. Fu, Int. J. Hydrogen Energy, 33 (2008) 6484-6491; (b) Y.-X. Li, Y.-Z. Xie, S.-Q. Peng, G.-X. Lu, S.-B. Li, Chem. J. Chin. Univ.-Chin., 28 (2007) 156-158.

[63] S.-Q. Peng, Y.-J. Peng, Y.-X. Li, G.-X. Lu, S.-B. Li, Res. Chem. Intermed., 35 (2009) 739749.

[64] S. Peng, M. Ding, T. Yi, Z. Zhan, Y. Li, Environ. Prog. Sustainable Energy, (2015) Ahead of Print.

[65] T. Chen, Z. Feng, G. Wu, J. Shi, G. Ma, P. Ying, C. Li, J. Phys. Chem. C, 111 (2007) 80058014.

[66] (a) M.R. Hoffmann, S.T. Martin, W. Choi, D.W. Bahnemann, Chem. Rev., 95 (1995) 6996; (b) J.R. Harbour, M.L. Hair, J. Phys. Chem., 83 (1979) 652-656; (c) J.R. Harbour, J. Tromp, M.L. Hair, Can. J. Chem., 63 (1985) 204-208; (d) F. Shiraishi, T. Nakasako, Z. Hua, J. Phys. Chem. A, 107 (2003) 11072-11081.

[67] C.-y. Wang, J. Rabani, D.W. Bahnemann, J.K. Dohrmann, J. Photochem. Photobiol., A, 148 (2002) 169-176.

[68] (a) Y. Nosaka, K. Koenuma, K. Ushida, A. Kira, Langmuir, 12 (1996) 736-738; (b) R.B. Draper, M.A. Fox, Langmuir, 6 (1990) 1396-1402.

[69] M. Kawai, T. Kawai, S. Naito, K. Tamaru, Chem. Phys. Lett., 110 (1984) 58-62.

[70] F.H. Hussein, R. Rudham, J. Chem. Soc., Faraday Trans. 1, 83 (1987) 1631-1639.

[71] F.H. Hussein, R. Rudham, J. Chem. Soc., Faraday Trans. 1, 80 (1984) 2817-2825.

[72] (a) O.I. Micic, Y.N. Zhang, K.R. Cromack, A.D. Trifunac, M.C. Thurnauer, J. Phys. Chem., 97 (1993) 7277-7283; (b) O.I. Micic, Y.N. Zhang, K.R. Cromack, A.D. Trifunac, M.C. Thurnauer, J. Phys. Chem., 97 (1993) 13284-13288.

[73] R.F. Howe, M. Grätzel, J. Phys. Chem., 91 (1987) 3906-3909.

[74] M. Anpo, T. Shima, Y. Kubokawa, Chem. Lett., (1985) 1799-1802. 
[75] S.I. Nishimoto, B. Ohtani, T. Kagiya, J. Chem. Soc., Perkin Trans. 1, 81 (1985) 2467-2474.

[76] T.A. Kandiel, R. Dillert, L. Robben, D.W. Bahnemann, Catal. Today, 161 (2011) 196-201.

[77] G.L. Chiarello, D. Ferri, E. Selli, J. Catal., 280 (2011) 168-177.

[78] A.Y. Nosaka, T. Fujiwara, H. Yagi, H. Akutsu, Y. Nosaka, Langmuir, 19 (2003) 1935-

1937.

[79] C.-Y. Wang, H. Groenzin, M.J. Shultz, J. Am. Chem. Soc., 126 (2004) 8094-8095.

[80] (a) Q. Gu, X. Fu, X. Wang, S. Chen, D.Y.C. Leung, X. Xie, Appl. Catal., B, 106 (2011)

689-696; (b) J. Araña, A.P. Alonso, J.M.D. Rodriguez, G. Colón, J.A. Navío, J.P. Peña, Appl.

Catal., B, 89 (2009) 204-213; (c) J. Araña, J.M. Doña-Rodríguez, O. González-Díaz, E. Tello

Rendón, J.A. Herrera Melián, G. Colón, J.A. Navío, J. Pérez Peña, J. Mol. Catal. A: Chem., 215 (2004) 153-160; (d) J. Araña, J.M. Doña-Rodríguez, C.G.I. Cabo, O. González-Díaz, J.A. HerreraMelián, J. Pérez-Peña, Appl. Catal., B, 53 (2004) 221-232; (e) J.M. Coronado, S. Kataoka, I. Tejedor-Tejedor, M.A. Anderson, J. Catal., 219 (2003) 219-230; (f) M. Kawai, S. Naito, K. Tamaru, T. Kawai, Chem. Phys. Lett., 98 (1983) 377-380; (g) L.F. Liao, W.C. Wu, C.Y. Chen, J.L. Lin, J. Phys. Chem. B, 105 (2001) 7678-7685.

[81] Y. Li, G. Lu, S. Li, Chemosphere, 52 (2003) 843-850.

[82] G.N. Ekström, A.J. McQuillan, J. Phys. Chem. B, 103 (1999) 10562-10565.

[83] T. Awatani, K.D. Dobson, A.J. McQuillan, B. Ohtani, K. Uosaki, Chem. Lett., (1998) 849-

850.

[84] H. Harada, T. Ueda, T. Sakata, J. Phys. Chem., 93 (1989) 1542-1548.

[85] (a) M. Kaise, H. Nagai, K. Tokuhashi, S. Kondo, S. Nimura, O. Kikuchi, Langmuir, 10 (1994) 1345-1347; (b) I.A. Shkrob, T.W. Marin, H. He, P. Zapol, J. Phys. Chem. C, 116 (2012) 9450-9460.

[86] T. Chen, G. Wu, Z. Feng, G. Hu, W. Su, P. Ying, C. Li, Chin. J. Catal., 29 (2008) 105-107. [87] S. Sato, K. Ueda, Y. Kawasaki, R. Nakamura, J. Phys. Chem. B, 106 (2002) 9054-9058.

[88] (a) B. Kraeutler, C.D. Jaeger, A.J. Bard, J. Am. Chem. Soc., 100 (1978) 4903-4905; (b) M. Kaise, H. Kondoh, C. Nishihara, H. Nozoye, H. Shindo, S. Nimura, O. Kikuchi, J. Chem. Soc.Chem. Commun., (1993) 395-396.

[89] (a) B. Kraeutler, A.J. Bard, J. Am. Chem. Soc., 100 (1978) 5985-5992; (b) H. Yoneyama, Y. Takao, H. Tamura, A.J. Bard, J. Phys. Chem., 87 (1983) 1417-1422.

[90] (a) H. Bahruji, M. Bowker, P.R. Davies, L.S. Al-Mazroai, A. Dickinson, J. Greaves, D. James, L. Millard, F. Pedrono, J. Photochem. Photobiol., A, 216 (2010) 115-118; (b) H. Bahruji, M. Bowker, P.R. Davies, F. Pedrono, Appl. Catal., B, 107 (2011) 205-209.

[91] (a) M. Bowker, D. James, P. Stone, R. Bennett, N. Perkins, L. Millard, J. Greaves, A. Dickinson, J. Catal., 217 (2003) 427-433; (b) M. Bowker, L. Millard, J. Greaves, D. James, J. Soares, Gold Bull., 37 (2004) 170-173; (c) M. Bowker, Catal. Lett., 142 (2012) 923-929.

[92] F. Sastre, M. Oteri, A. Corma, H. Garcia, Energy Environ. Sci., 6 (2013) 2211-2215.

[93] L. Millard, M. Bowker, J. Photochem. Photobiol. A-Chem., 148 (2002) 91-95.

[94] (a) G. Wu, T. Chen, W. Su, G. Zhou, X. Zong, Z. Lei, C. Li, Int. J. Hydrogen Energy, 33 (2008) 1243-1251; (b) Q. Xu, Y. Ma, J. Zhang, X. Wang, Z. Feng, C. Li, J. Catal., 278 (2011) 329335; (c) G. Wu, T. Chen, X. Zong, H. Yan, G. Ma, X. Wang, Q. Xu, D. Wang, Z. Lei, C. Li, J. Catal., 253 (2008) 225-227.

[95] G. Wu, T. Chen, G. Zhou, X. Zong, C. Li, Sci. China Ser. B-Chem., 51 (2008) 97-100.

[96] X. Fu, X. Wang, D.Y.C. Leung, Q. Gu, S. Chen, H. Huang, Appl. Catal., B, 106 (2011) 681-688.

[97] (a) H. Lu, J. Zhao, L. Li, L. Gong, J. Zheng, L. Zhang, Z. Wang, J. Zhang, Z. Zhu, Energy Environ. Sci., 4 (2011) 3384-3388; (b) L.H. Chen, W.Z. Gu, X.W. Zhu, F.D. Wang, Y.Z. Song, J.H. Hu, J. Photochem. Photobiol. A-Chem., 74 (1993) 85-89.

[98] N. Strataki, V. Bekiari, D.I. Kondarides, P. Lianos, Appl. Catal., B, 77 (2007) 184-189.

[99] W. Zhang, Y. Wang, Z. Wang, Z. Zhong, R. Xu, Chem. Commun., 46 (2010) 7631-7633. 
[100] (a) A.V. Puga, A. Forneli, H. Garcia, A. Corma, Adv. Funct. Mater., 24 (2014) 241-248; (b) T. Kawai, T. Sakata, J. Chem. Soc., Chem. Commun., (1980) 694-695; (c) G.L. Chiarello, L. Forni, E. Selli, Catal. Today, 144 (2009) 69-74; (d) K. Iseda, Bull. Chem. Soc. Jpn., 64 (1991) 1160-1166; (e) Y. Taniguchi, H. Yoneyama, H. Tamura, Chem. Lett., (1983) 269-272; (f) A. Gallo, T. Montini, M. Marelli, A. Minguzzi, V. Gombac, R. Psaro, P. Fornasiero, V. Dal Santo, ChemSusChem, 5 (2012) 1800-1811; (g) A. Gallo, M. Marelli, R. Psaro, V. Gombac, T. Montini, P. Fornasiero, R. Pievo, V. Dal Santo, Green Chem., 14 (2012) 330-333.

[101] I. Willner, Z. Goren, J. Chem. Soc.-Chem. Commun., (1986) 172-173.

[102] D. Jing, M. Liu, J. Shi, W. Tang, L. Guo, Catal. Commun., 12 (2010) 264-267.

[103] G.L. Chiarello, E. Selli, L. Forni, Appl. Catal., B, 84 (2008) 332-339.

[104] P. Pichat, M.N. Mozzanega, J. Disdier, J.M. Herrmann, Nouv. J. Chim., 6 (1982) 559-564.

[105] S. Martha, P. Chandra Sahoo, K.M. Parida, RSC Adv., 5 (2015) 61535-61553.

[106] A. Fujishima, K. Honda, Nature, 238 (1972) 37-38.

[107] (a) B. Kraeutler, A.J. Bard, J. Am. Chem. Soc., 100 (1978) 2239-2240; (b) A.J. Bard, J. Photochem., 10 (1979) 59-75.

[108] (a) T. Kawai, T. Sakata, Chem. Lett., (1981) 81-84; (b) T. Sakata, T. Kawai, Nouv. J. Chim., 5 (1981) 279-281; (c) S. Sato, J. Chem. Soc., Chem. Commun., (1982) 26-27; (d) E. Borgarello, E. Pelizzetti, Chim. Ind., 65 (1983) 474-478.

[109] P. Montes-Navajas, M. Serra, A. Corma, H. Garcia, Catal. Today, 225 (2014) 52-54.

[110] R.S. Davidson, C.L. Morrison, J. Abraham, J. Photochem., 24 (1984) 27-35.

[111] (a) M. Cargnello, T.R. Gordon, C.B. Murray, Chem. Rev., 114 (2014) 9319-9345; (b) X. Chen, S.S. Mao, Chem. Rev., 107 (2007) 2891-2959.

[112] M.-S. Park, M. Kang, Mater. Lett., 62 (2008) 183-187.

[113] (a) J. Jitputti, Y. Suzuki, S. Yoshikawa, Catal. Commun., 9 (2008) 1265-1271; (b) H.-L. Kuo, C.-Y. Kuo, C.-H. Liu, J.-H. Chao, C.-H. Lin, Catal. Lett., 113 (2007) 7-12.

[114] M. Murdoch, G.I.N. Waterhouse, M.A. Nadeem, J.B. Metson, M.A. Keane, R.F. Howe, J. Llorca, H. Idriss, Nat. Chem., 3 (2011) 489-492.

[115] W.-T. Chen, A. Chan, Z.H.N. Al-Azri, A.G. Dosado, M.A. Nadeem, D. Sun-Waterhouse, H. Idriss, G.I.N. Waterhouse, J. Catal., 329 (2015) 499-513.

[116] (a) B. Ohtani, J. Handa, S. Nishimoto, T. Kagiya, Chem. Phys. Lett., 120 (1985) 292-294; (b) G.L. Chiarello, A. Di Paola, L. Palmisano, E. Selli, Photochem. Photobiol. Sci., 10 (2011) 355360 .

[117] Á. Kmetykó, Á. Szániel, C. Tsakiroglou, A. Dombi, K. Hernádi, React. Kinet., Mech. Catal., (2015) Ahead of Print.

[118] D. Reyes-Coronado, G. Rodriguez-Gattorno, M.E. Espinosa-Pesqueira, C. Cab, R. de Coss, G. Oskan, Nanotechnology, 19 (2008) 145605/145601-145605/145610.

[119] J. Abrahams, R.S. Davidson, C.L. Morrison, J. Photochem., 29 (1985) 353-361.

[120] (a) W. Sun, S. Zhang, Z. Liu, C. Wang, Z. Mao, Int. J. Hydrogen Energy, 33 (2008) 1112-

1117; (b) J.-J. Zou, C.-J. Liu, K.-L. Yu, D.-G. Cheng, Y.-P. Zhang, F. He, H.-Y. Du, L. Cui, Chem. Phys. Lett., 400 (2004) 520-523; (c) C. Ampelli, R. Passalacqua, C. Genovese, S. Perathoner, G.

Centi, T. Montini, V. Gombac, J.J. Delgado Jaen, P. Fornasiero, RSC Adv., 3 (2013) 21776-21788; (d) J.M. Valero, S. Obregon, G. Colón, ACS Catal., 4 (2014) 3320-3329.

[121] (a) J. Yu, L. Qi, M. Jaroniec, J. Phys. Chem. C, 114 (2010) 13118-13125; (b) T.A. Kandiel, A. Feldhoff, L. Robben, R. Dillert, D.W. Bahnemann, Chem. Mater., 22 (2010) 2050-2060.

[122] X. Yang, L. Wu, L. Du, X. Li, Catal. Lett., 145 (2015) 1771-1777.

[123] (a) H.G. Yang, C.H. Sun, S.Z. Qiao, J. Zou, G. Liu, S.C. Smith, H.M. Cheng, G.Q. Lu, Nature, 453 (2008) 638-U634; (b) T. Montini, M. Monai, A. Beltram, I. Romero-Ocana, P. Fornasiero, Mater. Sci. Semicond. Process., (2015) Ahead of Print.

[124] I. Romero Ocaña, A. Beltram, J.J. Delgado Jaén, G. Adami, T. Montini, P. Fornasiero, Inorg. Chim. Acta, 431 (2015) 197-205. 
[125] S. Yurdakal, B.S. Tek, O. Alagöz, V. Augugliaro, V. Loddo, G. Palmisano, L. Palmisano, ACS Sustainable Chem. Eng., 1 (2013) 456-461.

[126] A. Beltram, I. Romero-Ocana, J.J.D. Jaen, T. Montini, P. Fornasiero, Appl. Catal., A, (2015) Ahead of Print.

[127] K. Tomita, V. Petrykin, M. Kobayashi, M. Shiro, M. Yoshimura, M. Kakihana, Angew. Chem., Int. Ed., 45 (2006) 2378-2381.

[128] (a) D. Praveen Kumar, N. Lakshmana Reddy, M. Mamatha Kumari, B. Srinivas, V. Durga Kumari, B. Sreedhar, V. Roddatis, O. Bondarchuk, M. Karthik, B. Neppolian, M.V. Shankar, Sol. Energy Mater. Sol. Cells, 136 (2015) 157-166; (b) C.-H. Lin, J.-H. Chao, C.-H. Liu, J.-C. Chang, F.-C. Wang, Langmuir, 24 (2008) 9907-9915; (c) F.-C. Wang, C.-H. Liu, C.-W. Liu, J.-H. Chao, C.-H. Lin, J. Phys. Chem. C, 113 (2009) 13832-13840; (d) C. Wang, X. Zhang, Y. Wei, L. Kong, F. Chang, H. Zheng, L. Wu, J. Zhi, Y. Liu, Dalton Trans., 44 (2015) 13331-13339.

[129] M.-C. Wu, J. Hiltunen, A. Sapi, A. Avila, W. Larsson, H.-C. Liao, M. Huuhtanen, G. Toth, A. Shchukarev, N. Laufer, A. Kukovecz, Z. Konya, J.-P. Mikkola, R. Keiski, W.-F. Su, Y.-F. Chen, H. Jantunen, P.M. Ajayan, R. Vajtai, K. Kordas, ACS Nano, 5 (2011) 5025-5030.

[130] A.G. Dosado, W.-T. Chen, A. Chan, D. Sun-Waterhouse, G.I.N. Waterhouse, J. Catal., 330 (2015) 238-254.

[131] G. Xiang, T. Li, J. Zhuang, X. Wang, Chem. Commun., 46 (2010) 6801-6803.

[132] H. Harada, T. Ueda, Chem. Phys. Lett., 106 (1984) 229-231.

[133] O. Rosseler, M.V. Shankar, M. Karkmaz-Le Du, L. Schmidlin, N. Keller, V. Keller, J. Catal., 269 (2010) 179-190.

[134] K. Lee, A. Mazare, P. Schmuki, Chem. Rev., 114 (2014) 9385-9454.

[135] X. Wang, Z. Li, J. Shi, Y. Yu, Chem. Rev., 114 (2014) 9346-9384.

[136] L. Wang, T. Sasaki, Chem. Rev., 114 (2014) 9455-9486.

[137] D. Fattakhova-Rohlfing, A. Zaleska, T. Bein, Chem. Rev., 114 (2014) 9487-9558.

[138] G. Liu, H.G. Yang, J. Pan, Y.Q. Yang, G.Q. Lu, H.-M. Cheng, Chem. Rev., 114 (2014) 9559-9612.

[139] D.M. Antonelli, J.Y. Ying, Angew. Chem., Int. Ed. Engl., 34 (1995) 2014-2017.

[140] (a) J. Jitputti, S. Pavasupree, Y. Suzuki, S. Yoshikawa, J. Solid State Chem., 180 (2007) 1743-1749; (b) T. Sreethawong, T. Puangpetch, S. Chavadej, S. Yoshikawa, J. Power Sources, 165 (2007) 861-869; (c) M.I. Badawy, M.Y. Ghaly, M.E.M. Ali, Desalination, 267 (2011) 250-255.

[141] J. Puskelova, L. Baia, A. Vulpoi, M. Baia, M. Antoniadou, V. Dracopoulos, E. Stathatos, K. Gabor, Z. Pap, V. Danciu, P. Lianos, Chem. Eng. J., 242 (2014) 96-101.

[142] S. Chuangchote, J. Jitputti, T. Sagawa, S. Yoshikawa, ACS Appl. Mater. Interfaces, 1 (2009) 1140-1143.

[143] (a) N.T. Nguyen, M. Altomare, J.E. Yoo, P. Schmuki, Adv. Mater., 27 (2015) 3208-3215;

(b) C. Ampelli, G. Centi, R. Passalacqua, S. Perathoner, Energy Environ. Sci., 3 (2010) 292-301; (c)

A. Ghicov, H. Tsuchiya, J.M. Macak, P. Schmuki, Electrochem. Commun., 7 (2005) 505-509; (d)

D. Gong, C.A. Grimes, O.K. Varghese, W. Hu, R.S. Singh, Z. Chen, E.C. Dickey, J. Mater. Res., 16 (2001) 3331-3334; (e) C.A. Grimes, J. Mater. Chem., 17 (2007) 1451-1457; (f) H.E. Prakasam, K. Shankar, M. Paulose, O.K. Varghese, C.A. Grimes, J. Phys. Chem. C, 111 (2007) 7235-7241; (g) J.M. Macak, H. Tsuchiya, L. Taveira, S. Aldabergerova, P. Schmuki, Angew. Chem., Int. Ed., 44 (2005) 7463-7465.

[144] S. Zhang, B. Peng, S. Yang, Y. Fang, F. Peng, Int. J. Hydrogen Energy, 38 (2013) 1386613871.

[145] X. Fan, J. Fan, X.Y. Hu, E.Z. Liu, L.M. Kang, C.N. Tang, Y.N. Ma, H.T. Wu, Y.Y. Li, Ceram. Int., 40 (2014) 15907-15917.

[146] in, 2015.

[147] Y.K. Kho, A. Iwase, W.Y. Teoh, L. Madler, A. Kudo, R. Amal, J. Phys. Chem. C, 114 (2010) 2821-2829. 
[148] B.W. Robinson, C.J. Tighe, R.I. Gruar, A. Mills, I.P. Parkin, A.K. Tabecki, H.L. de Villiers Lovelock, J.A. Darr, J. Mater. Chem. A, 3 (2015) 12680-12689.

[149] (a) G. Li, K.A. Gray, Chem. Phys., 339 (2007) 173-187; (b) T. Kawahara, Y. Konishi, H. Tada, N. Tohge, J. Nishii, S. Ito, Angew. Chem., Int. Ed., 41 (2002) 2811-2813; (c) J. Zhang, Q. Xu, Z. Feng, M. Li, C. Li, Angew. Chem., Int. Ed., 47 (2008) 1766-1769; (d) T. Ohno, K. Sarukawa, K. Tokieda, M. Matsumura, J. Catal., 203 (2001) 82-86.

[150] V. Jovic, W.-T. Chen, D. Sun-Waterhouse, M.G. Blackford, H. Idriss, G.I.N. Waterhouse, J. Catal., 305 (2013) 307-317.

[151] D.O. Scanlon, C.W. Dunnill, J. Buckeridge, S.A. Shevlin, A.J. Logsdail, S.M. Woodley, C.R.A. Catlow, M.J. Powell, R.G. Palgrave, I.P. Parkin, G.W. Watson, T.W. Keal, P. Sherwood, A. Walsh, A.A. Sokol, Nat. Mater., 12 (2013) 798-801.

[152] S. Bashir, A.K. Wahab, H. Idriss, Catal. Today, 240 (2015) 242-247.

[153] (a) M. Reza Gholipour, C.-T. Dinh, F. Beland, T.-O. Do, Nanoscale, 7 (2015) 8187-8208;

(b) D.Y.C. Leung, X. Fu, C. Wang, M. Ni, M.K.H. Leung, X. Wang, X. Fu, ChemSusChem, 3 (2010) 681-694; (c) M. Dahl, Y. Liu, Y. Yin, Chem. Rev., 114 (2014) 9853-9889.

[154] (a) N. Strataki, M. Antoniadou, V. Dracopoulos, P. Lianos, Catal. Today, 151 (2010) 53-57;

(b) V.M. Daskalaki, M. Antoniadou, G. Li Puma, D.I. Kondarides, P. Lianos, Environ. Sci.

Technol., 44 (2010) 7200-7205.

[155] L. Qi, J. Yu, M. Jaroniec, Phys. Chem. Chem. Phys., 13 (2011) 8915-8923.

[156] V. Thi Thuy Duong, F. Mighri, A. Ajji, D. Trong-On, Ind. Eng. Chem. Res., 53 (2014) 3888-3897.

[157] (a) D. Cao-Thang, P. Minh-Hao, F. Kleitz, D. Trong-On, J. Mater. Chem. A, 1 (2013) 13308-13313; (b) D. Cao-Thang, P. Minh-Hao, Y. Seo, F. Kleitz, D. Trong-On, Nanoscale, 6 (2014) 4819-4829.

[158] (a) W. Fan, Q. Lai, Q. Zhang, Y. Wang, J. Phys. Chem. C, 115 (2011) 10694-10701; (b) Q. Xiang, J. Yu, J. Phys. Chem. Lett., 4 (2013) 753-759.

[159] (a) C.G. Silva, M.J. Sampaio, R.R.N. Marques, L.A. Ferreira, P.B. Tavares, A.M.T. Silva, J.L. Faria, Appl. Catal., B, 178 (2015) 82-90; (b) A. Moya, A. Cherevan, S. Marchesan, P. Gebhardt, M. Prato, D. Eder, J.J. Vilatela, Appl. Catal., B, 179 (2015) 574-582.

[160] M. Melchionna, A. Beltram, T. Montini, M. Monai, L. Nasi, P. Fornasiero, M. Prato, Chem. Commun., (2015) 10.1039/c1035cc08015k.

[161] (a) S. Sato, J.M. White, Ind. Eng. Chem. Prod. Res. Dev., 19 (1980) 542-544; (b) K. Hashimoto, T. Kawai, T. Sakata, J. Phys. Chem., 88 (1984) 4083-4088; (c) T. Kawai, T. Sakata, Nature, 282 (1979) 283-284; (d) T. Kawai, T. Sakata, J. Chem. Soc.-Chem. Commun., (1979) $1047-$ 1048; (e) S. Sato, J.M. White, J. Phys. Chem., 85 (1981) 336-341; (f) H. Yoshida, K. Hirao, J.I. Nishimoto, K. Shimura, S. Kato, H. Itoh, T. Hattori, J. Phys. Chem. C, 112 (2008) 5542-5551. [162] D.A. Neamen, Semiconductor Physics and Devices: Basic Principles, $3^{\text {rd }}$ ed., McGraw-Hill Education, New York, 2003.

[163] J. Highfield, Molecules, 20 (2015) 6739-6793.

[164] (a) X. Li, J. Yu, J. Low, Y. Fang, J. Xiao, X. Chen, J. Mater. Chem. A, 3 (2015) 2485-2534; (b) L. Sang, Y. Zhao, C. Burda, Chem. Rev., 114 (2014) 9283-9318; (c) R. Asahi, T. Morikawa, H. Irie, T. Ohwaki, Chem. Rev., 114 (2014) 9824-9852.

[165] T. Sun, J. Fan, E. Liu, L. Liu, Y. Wang, H. Dai, Y. Yang, W. Hou, X. Hu, Z. Jiang, Powder Technol., 228 (2012) 210-218.

[166] T. Sun, E. Liu, X. Liang, X. Hu, J. Fan, Appl. Surf. Sci., 347 (2015) 696-705.

[167] S. Taylor, M. Mehta, A. Samokhvalov, ChemPhysChem, 15 (2014) 942-949.

[168] S. Asal, M. Saif, H. Hafez, S. Mozia, A. Heciak, D. Moszynski, M.S.A. Abdel-Mottaleb, Int. J. Hydrogen Energy, 36 (2011) 6529-6537.

[169] R. Asahi, T. Morikawa, T. Ohwaki, K. Aoki, Y. Taga, Science, 293 (2001) 269-271.

[170] C. Di Valentin, G. Pacchioni, Catal. Today, 206 (2013) 12-18.

[171] H. Irie, Y. Watanabe, K. Hashimoto, J. Phys. Chem. B, 107 (2003) 5483-5486. 
[172] Y. Kuo, K.J. Klabunde, Appl. Catal., B, 104 (2011) 245-251.

[173] H. Irie, Y. Watanabe, K. Hashimoto, Chem. Lett., 32 (2003) 772-773.

[174] P. Carmichael, D. Hazafy, D.S. Bhachu, A. Mills, J.A. Darr, I.P. Parkin, Phys. Chem.

Chem. Phys., 15 (2013) 16788-16794.

[175] Y. Tian, H. Sang, X. Wang, Chin. J. Catal., 33 (2012) 1395-1401.

[176] M. Yoshinaga, K. Yamamoto, N. Sato, K. Aoki, T. Morikawa, A. Muramatsu, Appl. Catal., B, 87 (2009) 239-244.

[177] K. Nishijima, T. Kamai, N. Murakami, T. Tsubota, T. Ohno, Int. J. Photoenergy, (2008) 173943.

[178] (a) X. Chen, L. Liu, P.Y. Yu, S.S. Mao, Science, 331 (2011) 746-750; (b) X. Chen, L. Liu, F. Huang, Chem. Soc. Rev., 44 (2015) 1861-1885.

[179] F. Zuo, L. Wang, T. Wu, Z. Zhang, D. Borchardt, P. Feng, J. Am. Chem. Soc., 132 (2010) 11856-11857.

[180] J. Jiang, H. Zhou, J. Ding, F. Zhang, T. Fan, D. Zhang, Int. J. Hydrogen Energy, 40 (2015) 9155-9164.

[181] B. Han, Y.H. Hu, J. Phys. Chem. C, 119 (2015) 18927-18934.

[182] A. Sobczynski, A.J. Bard, A. Campion, M.A. Fox, T. Mallouk, S.E. Webber, J.M. White, J. Phys. Chem., 91 (1987) 3316-3320.

[183] R. Abe, H. Takami, N. Murakami, B. Ohtani, J. Am. Chem. Soc., 130 (2008) 7780-+.

[184] K. Domen, S. Naito, T. Onishi, K. Tamaru, Chem. Lett., (1982) 555-558.

[185] M. Ashokkumar, Int. J. Hydrogen Energy, 23 (1998) 427-438.

[186] A. Tanaka, K. Hashimoto, H. Kominami, J. Am. Chem. Soc., 136 (2014) 586-589.

[187] J. Long, W. Xue, X. Xie, Q. Gu, Y. Zhou, Y. Chi, W. Chen, Z. Ding, X. Wang, Catal. Commun., 16 (2011) 215-219.

[188] H. Liu, K. Zhao, T. Wang, J. Deng, H. Zeng, Mater. Sci. Semicond. Process., 40 (2015) 670-675.

[189] S.-y. Guo, T.-j. Zhao, Z.-q. Jin, X.-m. Wan, P.-g. Wang, J. Shang, S. Han, J. Power Sources, 293 (2015) 17-22.

[190] R. Lv, X. Wang, W. Lv, Y. Xu, Y. Ge, H. He, G. Li, X. Wu, X. Li, Q. Li, J. Chem. Technol. Biotechnol., 90 (2015) 550-558.

[191] (a) D. Bao, P. Gao, X. Zhu, S. Sun, Y. Wang, X. Li, Y. Chen, H. Zhou, Y. Wang, P. Yang, Chemistry - A European Journal, 21 (2015) 12728-12734; (b) H.X. Sang, X.T. Wang, C.C. Fan, F. Wang, Int. J. Hydrogen Energy, 37 (2012) 1348-1355.

[192] A. Hameed, M.A. Gondal, J. Mol. Catal. A: Chem., 233 (2005) 35-41.

[193] S. Kakuta, T. Abe, ACS Appl. Mater. Interfaces, 1 (2009) 2707-2710.

[194] D. Barreca, P. Fornasiero, A. Gasparotto, V. Gombac, C. Maccato, T. Montini, E. Tondello, ChemSusChem, 2 (2009) 230-233.

[195] Q. Simon, D. Barreca, A. Gasparotto, C. Maccato, T. Montini, V. Gombac, P. Fornasiero, O.I. Lebedev, S. Turner, G. Van Tendeloo, J. Mater. Chem., 22 (2012) 11739-11747.

[196] (a) G. Carraro, C. Maccato, A. Gasparotto, T. Montini, S. Turner, O.I. Lebedev, V.

Gombac, G. Adami, G. Van Tendeloo, D. Barreca, P. Fornasiero, Adv. Funct. Mater., 24 (2014) 372-378; (b) G. Carraro, A. Gasparotto, C. Maccato, V. Gombac, F. Rossi, T. Montini, D. Peeters, E. Bontempi, C. Sada, D. Barreca, P. Fornasiero, RSC Adv., 4 (2014) 32174-32179.

[197] A. Gasparotto, D. Barreca, D. Bekermann, A. Devi, R.A. Fischer, P. Fornasiero, V. Gombac, O.I. Lebedev, C. Maccato, T. Montini, G. Van Tendeloo, E. Tondello, J. Am. Chem. Soc., 133 (2011) 19362-19365.

[198] Y. Wang, Z. Zhang, Y. Zhu, Z. Li, R. Vajtai, L. Ci, P.M. Ajayan, ACS Nano, 2 (2008) 1492-1496.

[199] W. Wang, M.O. Tade, Z. Shao, Chem. Soc. Rev., 44 (2015) 5371-5408.

[200] J. Peng, Y. Zhou, H. Wang, H. Zhou, S. Cai, CrystEngComm, 17 (2015) 1805-1812. 
[201] (a) T. Puangpetch, T. Sreethawong, S. Yoshikawa, S. Chavadej, J. Mol. Catal. A: Chem., 312 (2009) 97-106; (b) J.-P. Zou, L.-Z. Zhang, S.-L. Luo, L.-H. Leng, X.-B. Luo, M.-J. Zhang, Y. Luo, G.-C. Guo, Int. J. Hydrogen Energy, 37 (2012) 17068-17077; (c) A.K. Wahab, T. Odedairo, J. Labis, M. Hedhili, A. Delavar, H. Idriss, Appl. Petrochem. Res., 3 (2013) 83-89; (d) B. Zielinska, E. Borowiak-Palen, R.J. Kalenczuk, Int. J. Hydrogen Energy, 33 (2008) 1797-1802.

[202] K. Domen, A. Kudo, T. Onishi, J. Catal., 102 (1986) 92-98.

[203] W. Sun, S. Zhang, C. Wang, Z. Liu, Z. Mao, Catal. Lett., 119 (2007) 148-153.

[204] W. Sun, S. Zhang, C. Wang, Z. Liu, Z. Mao, Catal. Lett., 123 (2008) 282-288.

[205] Y. Xue, X. Wang, Int. J. Hydrogen Energy, 40 (2015) 5878-5888.

[206] X. Fu, X. Wang, D.Y.C. Leung, W. Xue, Z. Ding, H. Huang, X. Fu, Catal. Commun., 12 (2010) 184-187.

[207] (a) K. Domen, A. Kudo, A. Shinozaki, A. Tanaka, K. Maruya, T. Onishi, J. Chem. Soc., Chem. Commun., (1986) 356-357; (b) K. Domen, A. Kudo, M. Shibata, A. Tanaka, K. Maruya, T. Onishi, J. Chem. Soc.-Chem. Commun., (1986) 1706-1707; (c) A. Kudo, A. Tanaka, K. Domen, K. Maruya, K. Aika, T. Onishi, J. Catal., 111 (1988) 67-76.

[208] Y. Ebina, A. Tanaka, J.N. Kondo, K. Domen, Chem. Mater., 8 (1996) 2534-2538.

[209] M. Liu, W. You, Z. Lei, G. Zhou, J. Yang, G. Wu, G. Ma, G. Luan, T. Takata, M. Hara, K. Domen, C. Li, Chem. Commun., (2004) 2192-2193.

[210] R.P. Panmand, Y.A. Sethi, S.R. Kadam, M.S. Tamboli, L.K. Nikam, J.D. Ambekar, C.-J. Park, B.B. Kale, CrystEngComm, 17 (2015) 107-115.

[211] J. Li, X. Pan, Y. Xu, L. Jia, X. Yi, W. Fang, Int. J. Hydrogen Energy, 40 (2015) 1391813925.

[212] K. Zhang, L. Guo, Catal. Sci. Technol., 3 (2013) 1672-1690.

[213] (a) Z. Jin, Q. Li, X. Zheng, C. Xi, C. Wang, H. Zhang, L. Feng, H. Wang, Z. Chen, Z. Jiang, J. Photochem. Photobiol., A, 71 (1993) 85-96; (b) A.J. Bard, Science, 207 (1980) 139-144; (c) M. Ni, M.K.H. Leung, D.Y.C. Leung, K. Sumathy, Renewable Sustainable Energy Rev., 11 (2006) 401-425.

[214] T. Sakata, T. Kawai, J. Synth. Org. Chem., Jpn., 39 (1981) 589-602.

[215] H. Harada, T. Sakata, T. Ueda, J. Am. Chem. Soc., 107 (1985) 1773-1774.

[216] T. Sakata, J. Photochem., 29 (1985) 205-215.

[217] Z. Hu, J.C. Yu, J. Mater. Chem. A, 1 (2013) 12221-12228.

[218] M. Matsumura, M. Hiramoto, T. Iehara, H. Tsubomura, J. Phys. Chem., 88 (1984) 248-250.

[219] S.Y. Ryu, W. Balcerski, T.K. Lee, M.R. Hoffmann, J. Phys. Chem. C, 111 (2007) 18195-

18203.

[220] (a) Y. Li, Y. Hu, S. Peng, G. Lu, S. Li, J. Phys. Chem. C, 113 (2009) 9352-9358; (b) Y. Li, J. Du, S. Peng, D. Xi, G. Lu, S. Li, Int. J. Hydrogen Energy, 33 (2008) 2007-2013; (c) Y.J. Zhang, L. Zhang, Desalination, 249 (2009) 1017-1021.

[221] Q. Li, B. Guo, J. Yu, J. Ran, B. Zhang, H. Yan, J.R. Gong, J. Am. Chem. Soc., 133 (2011) 10878-10884.

[222] J.-J. Zhou, R. Wang, X.-L. Liu, F.-M. Peng, C.-H. Li, F. Teng, Y.-P. Yuan, Appl. Surf. Sci., 346 (2015) 278-283.

[223] (a) M.d.O. Melo, L.A. Silva, J. Photochem. Photobiol. A-Chem., 226 (2011) 36-41; (b)

S.A.L. Bastos, P.A.L. Lopes, F.N. Santos, L.A. Silva, Int. J. Hydrogen Energy, 39 (2014) 1458814595.

[224] X. Wang, W.-c. Peng, X.-y. Li, Int. J. Hydrogen Energy, 39 (2014) 13454-13461.

[225] (a) R. Peng, C.-M. Wu, J. Baltrusaitis, N.M. Dimitrijevic, T. Rajh, R.T. Koodali, Chem. Commun., 49 (2013) 3221-3223; (b) R. Peng, D. Zhao, J. Baltrusaitis, C.-M. Wu, R.T. Koodali, RSC Adv., 2 (2012) 5754-5767; (c) Y.J. Zhang, L. Zhang, S. Li, Int. J. Hydrogen Energy, 35 (2010) 438-444; (d) Y.J. Zhang, L. Zhang, Appl. Surf. Sci., 255 (2009) 4863-4866.

[226] L. Shen, M. Luo, Y. Liu, R. Liang, F. Jing, L. Wu, Appl. Catal., B, 166 (2015) 445-453. 
[227] J. Hu, A. Liu, H. Jin, D. Ma, D. Yin, P. Ling, S. Wang, Z. Lin, J. Wang, J. Am. Chem. Soc., (2015).

[228] Y. Li, D. Gao, S. Peng, G. Lu, S. Li, Int. J. Hydrogen Energy, 36 (2011) 4291-4297.

[229] P.A.L. Lopes, A.J.S. Mascarenhas, L.A. Silva, J. Alloys Compd., 649 (2015) 332-336.

[230] E.A. Kozlova, D.V. Markovskaya, S.V. Cherepanova, A.A. Saraev, E.Y. Gerasimov, T.V. Perevalov, V.V. Kaichev, V.N. Parmon, Int. J. Hydrogen Energy, 39 (2014) 18758-18769.

[231] (a) L.H. Chen, X.W. Zhu, F.D. Wang, W.Z. Gu, J. Photochem. Photobiol. A-Chem., 73

(1993) 217-220; (b) S. Yanagida, T. Azuma, H. Sakurai, Chem. Lett., (1982) 1069-1070.

[232] X. Wang, R. Lv, K. Wang, J. Mater. Chem. A, 2 (2014) 8304-8313.

[233] C. Li, Z. Xi, W. Fang, M. Xing, J. Zhang, J. Solid State Chem., 226 (2015) 94-100.

[234] Y. Peng, L. Shang, Y. Cao, Q. Wang, Y. Zhao, C. Zhou, T. Bian, L.-Z. Wu, C.-H. Tung, T. Zhang, Appl. Surf. Sci.

[235] S. Kambe, M. Fujii, T. Kawai, S. Kawai, F. Nakahara, Chem. Phys. Lett., 109 (1984) 105-

109.

[236] Z. Han, F. Qiu, R. Eisenberg, P.L. Holland, T.D. Krauss, Science, 338 (2012) 1321-1324.

[237] A. Das, Z. Han, M.G. Haghighi, R. Eisenberg, Proc. Natl. Acad. Sci. U.S.A., 110 (2013) 16716-16723.

[238] P. Wang, J. Zhang, H. He, X. Xu, Y. Jin, Nanoscale, 7 (2015) 5767-5775.

[239] (a) C. Gimbert-Surinach, J. Albero, T. Stoll, J. Fortage, M.-N. Collomb, A. Deronzier, E. Palomares, A. Llobet, J. Am. Chem. Soc., 136 (2014) 7655-7661; (b) K. Han, M. Wang, S. Zhang, S. Wu, Y. Yang, L. Sun, Chem. Commun., 51 (2015) 7008-7011.

[240] T. Inoue, A. Fujishima, S. Konishi, K. Honda, Nature, 277 (1979) 637-638.

[241] S. Hu, M.R. Shaner, J.A. Beardslee, M. Lichterman, B.S. Brunschwig, N.S. Lewis, Science, 344 (2014) 1005-1009.

[242] Q. Xiang, B. Cheng, J. Yu, Angew. Chem., Int. Ed., 54 (2015) 11350-11366.

[243] C. Lavorato, A. Primo, R. Molinari, H. Garcia, Chem. - Eur. J., 20 (2014) 187-194.

[244] M. Latorre-Sanchez, A. Primo, H. Garcia, Angew. Chem., Int. Ed., 52 (2013) 11813-11816.

[245] T.-F. Yeh, J.-M. Syu, C. Cheng, T.-H. Chang, H. Teng, Adv. Funct. Mater., 20 (2010) 2255-2262.

[246] (a) S. Ye, R. Wang, M.-Z. Wu, Y.-P. Yuan, Appl. Surf. Sci., (2015) Ahead of Print; (b) J. Liu, Y. Liu, N. Liu, Y. Han, X. Zhang, H. Huang, Y. Lifshitz, S.-T. Lee, J. Zhong, Z. Kang, Science, 347 (2015) 970-974.

[247] X. Wang, K. Maeda, A. Thomas, K. Takanabe, G. Xin, J.M. Carlsson, K. Domen, M. Antonietti, Nat. Mater., 8 (2009) 76-80.

[248] X. Zhang, L. Yu, C. Zhuang, T. Peng, R. Li, X. Li, ACS Catal., 4 (2014) 162-170.

[249] Y. Hou, A.B. Laursen, J. Zhang, G. Zhang, Y. Zhu, X. Wang, S. Dahl, I. Chorkendorff, Angew. Chem., Int. Ed., 52 (2013) 3621-3625.

[250] P.A. Mangrulkar, A.V. Kotkondawar, S. Mukherjee, M.V. Joshi, N. Labhsetwar, D.D.

Sarma, S.S. Rayalu, Energy Environ. Sci., 7 (2014) 4087-4094.

[251] J. Hong, Y. Wang, Y. Wang, W. Zhang, R. Xu, ChemSusChem, 6 (2013) 2263-2268.

[252] C.G. Silva, A. Corma, H. García, J. Mater. Chem., 20 (2010) 3141-3156.

[253] C.G. Silva, I. Luz, F.X. Llabres i Xamena, A. Corma, H. Garcia, Chem. - Eur. J., 16 (2010)

$11133-11138$.

[254] L. Stegbauer, K. Schwinghammer, B.V. Lotsch, Chem. Sci., 5 (2014) 2789-2793.

[255] X.H. Zhang, B.S. Peng, S. Zhang, T.Y. Peng, ACS Sustainable Chem. Eng., 3 (2015) 1501-

1509 .

[256] J. Yang, D. Wang, H. Han, C. Li, Acc. Chem. Res., 46 (2013) 1900-1909.

[257] (a) J. Ran, J. Zhang, J. Yu, M. Jaroniec, S.Z. Qiao, Chem. Soc. Rev., 43 (2014) 7787-7812;

(b) Y. Xu, R. Xu, Appl. Surf. Sci., 351 (2015) 779-793.

[258] S.R. Soltau, J. Niklas, P.D. Dahlberg, O.G. Poluektov, D.M. Tiede, K.L. Mulfort, L.M.

Utschig, Chem. Commun., 51 (2015) 10628-10631. 
[259] (a) Y. Xu, B. Zhang, Catal. Sci. Technol., 5 (2015) 3084-3096; (b) S. Fukuzumi, Curr. Opin. Chem. Biol., 25 (2015) 18-26.

[260] A.J. Bard, J. Phys. Chem., 86 (1982) 172-177.

[261] B. Kraeutler, A.J. Bard, J. Am. Chem. Soc., 100 (1978) 4317-4318.

[262] G.R. Bamwenda, S. Tsubota, T. Nakamura, M. Haruta, J. Photochem. Photobiol., A, 89

(1995) 177-189.

[263] L.S. Al-Mazroai, M. Bowker, P. Davies, A. Dickinson, J. Greaves, D. James, L. Millard, Catal. Today, 122 (2007) 46-50.

[264] J. Greaves, L. Al-Mazroai, A. Nuhu, P. Davies, M. Bowker, Gold Bull., 39 (2006) 216-219.

[265] Z.H.N. Al-Azri, W.-T. Chen, A. Chan, V. Jovic, T. Ina, H. Idriss, G.I.N. Waterhouse, J.

Catal., 329 (2015) 355-367.

[266] V. Jovic, Z.H.N. Al-Azri, W.-T. Chen, D. Sun-Waterhouse, H. Idriss, G.I.N. Waterhouse, Top. Catal., 56 (2013) 1139-1151.

[267] A. Primo, A. Corma, H. García, Phys. Chem. Chem. Phys., 13 (2011) 886-910.

[268] (a) Z.H.N. Al-Azri, V. Jovic, W.-T. Chen, D. Sun-Waterhouse, J.B. Metson, G.I.N.

Waterhouse, Int. J. Nanotechnol., 11 (2014) 695-703; (b) C. Ampelli, C. Genovese, R. Passalacqua, S. Perathoner, G. Centi, Appl. Therm. Eng., 70 (2014) 1270-1275; (c) G.R. Bamwenda, S. Tsubota, T. Kobayashi, M. Haruta, J. Photochem. Photobiol., A, 77 (1994) 59-67.

[269] (a) M. Serra, J. Albero, H. Garcia, ChemPhysChem, 16 (2015) 1842-1845; (b) H.G.

Baldovi, F. Albarracin, P. Atienzar, B. Ferrer, M. Alvaro, H. Garcia, ChemPhysChem, 16 (2015) 335-341.

[270] K. Lee, R. Hahn, P. Schmuki, Electrochem. Commun., 43 (2014) 105-108.

[271] P.C.K. Vesborg, T.F. Jaramillo, RSC Adv., 2 (2012) 7933-7947.

[272] (a) V. Gombac, L. Sordelli, T. Montini, J.J. Delgado, A. Adamski, G. Adami, M. Cargnello, S. Bernal, P. Fornasiero, J. Phys. Chem. A, 114 (2010) 3916-3925; (b) A. Petala, E. Ioannidou, A. Georgaka, K. Bourikas, D.I. Kondarides, Appl. Catal., B, 178 (2015) 201-209; (c) K. Lalitha, G. Sadanandam, V.D. Kumari, M. Subrahmanyam, B. Sreedhar, N.Y. Hebalkar, J. Phys. Chem. C, 114 (2010) 22181-22189; (d) S. Xu, D.D. Sun, Int. J. Hydrogen Energy, 34 (2009) 6096-6104.

[273] W.-T. Chen, V. Jovic, D. Sun-Waterhouse, H. Idriss, G.I.N. Waterhouse, Int. J. Hydrogen Energy, 38 (2013) 15036-15048.

[274] J. Yu, Y. Hai, M. Jaroniec, J. Colloid Interface Sci., 357 (2011) 223-228.

[275] N.-L. Wu, M.-S. Lee, Int. J. Hydrogen Energy, 29 (2004) 1601-1605.

[276] D.W. Skaf, N.G. Natrin, K.C. Brodwater, C.R. Bongo, Catal. Lett., 142 (2012) 1175-1179.

[277] T. Montini, V. Gombac, L. Sordelli, J.J. Delgado, X. Chen, G. Adami, P. Fornasiero, ChemCatChem, 3 (2011) 574-577.

[278] W.-T. Chen, A. Chan, D. Sun-Waterhouse, T. Moriga, H. Idriss, G.I.N. Waterhouse, J. Catal., 326 (2015) 43-53.

[279] A.V. Korzhak, N.I. Ermokhina, A.L. Stroyuk, V.K. Bukhtiyarov, A.E. Raevskaya, V.I. Litvin, S.Y. Kuchmiy, V.G. Ilyin, P.A. Manorik, J. Photochem. Photobiol. A-Chem., 198 (2008) 126-134.

[280] S.-i. Fujita, H. Kawamori, D. Honda, H. Yoshida, M. Arai, Appl. Catal., B, 181 (2016) 818824.

[281] S. Cao, C.-J. Wang, X.-J. Lv, Y. Chen, W.-F. Fu, Appl. Catal., B, 162 (2015) 381-391.

[282] L. Mahoney, R. Peng, C.-M. Wu, J. Baltrusaitis, R.T. Koodali, Int. J. Hydrogen Energy, 40 (2015) 10795-10806.

[283] Q. Gu, J. Long, L. Fan, L. Chen, L. Zhao, H. Lin, X. Wang, J. Catal., 303 (2013) 141-155.

[284] A.K. Wahab, S. Bashir, Y. Al-Salik, H. Idriss, Appl. Petrochem. Res., 4 (2014) 55-62.

[285] G.I.N. Waterhouse, A.K. Wahab, M. Al-Oufi, V. Jovic, D.H. Anjum, D. Sun-Waterhouse, J. Llorca, H. Idriss, Sci. Rep., 3 (2013).

[286] M. Bowker, C. Morton, J. Kennedy, H. Bahruji, J. Greves, W. Jones, P.R. Davies, C. Brookes, P.P. Wells, N. Dimitratos, J. Catal., 310 (2014) 10-15. 
[287] R. Su, R. Tiruvalam, A.J. Logsdail, Q. He, C.A. Downing, M.T. Jensen, N. Dimitratos, L. Kesavan, P.P. Wells, R. Bechstein, H.H. Jensen, S. Wendt, C.R.A. Catlow, C.J. Kiely, G.J. Hutchings, F. Besenbacher, ACS Nano, 8 (2014) 3490-3497.

[288] E. Bonmatí, A. Casanovas, I. Angurell, J. Llorca, Top. Catal., 58 (2015) 77-84.

[289] T.R. Thurston, J.P. Wilcoxon, J. Phys. Chem. B, 103 (1999) 11-17.

[290] J. Kibsgaard, Z. Chen, B.N. Reinecke, T.F. Jaramillo, Nat. Mater., 11 (2012) 963-969.

[291] (a) G. Chen, D. Li, F. Li, Y. Fan, H. Zhao, Y. Luo, R. Yu, Q. Meng, Appl. Catal. A-Gen., 443 (2012) 138-144; (b) X. Zong, H. Yan, G. Wu, G. Ma, F. Wen, L. Wang, C. Li, J. Am. Chem. Soc., 130 (2008) 7176-7177.

[292] X. Zong, G. Wu, H. Yan, G. Ma, J. Shi, F. Wen, L. Wang, C. Li, J. Phys. Chem. C, 114 (2010) 1963-1968.

[293] X. Zong, J. Han, G. Ma, H. Yan, G. Wu, C. Li, J. Phys. Chem. C, 115 (2011) 12202-12208. [294] (a) Y. Li, H. Wang, S. Peng, J. Phys. Chem. C, 118 (2014) 19842-19848; (b) K. Chang, Z. Mei, T. Wang, Q. Kang, S. Ouyang, J. Ye, ACS Nano, 8 (2014) 7078-7087; (c) D. Lang, T. Shen, Q. Xiang, ChemCatChem, 7 (2015) 943-951; (d) Q. Xiang, J. Yu, M. Jaroniec, J. Am. Chem. Soc., 134 (2012) 6575-6578.

[295] S. Cao, Y. Chen, C.-J. Wang, P. He, W.-F. Fu, Chem. Commun., 50 (2014) 10427-10429.

[296] S. Cao, Y. Chen, C.-C. Hou, X.-J. Lv, W.-F. Fu, J. Mater. Chem. A, 3 (2015) 6096-6101.

[297] S. Cao, Y. Chen, C.-J. Wang, X.-J. Lv, W.-F. Fu, Chem. Commun., 51 (2015) 8708-8711.

[298] Q. Yue, Y. Wan, Z. Sun, X. Wu, Y. Yuan, P. Du, J. Mater. Chem. A, 3 (2015) 1694116947.

[299] S. Cao, Y. Chen, L. Kang, Z. Lin, W.-F. Fu, J. Mater. Chem. A, (2015).

[300] X. Wang, H. Yu, L. Yang, L. Shao, L. Xu, Catal. Commun., 67 (2015) 45-48.

[301] S.G. Babu, R. Vinoth, D.P. Kumar, M.V. Shankar, H.L. Chou, K. Vinodgopal, B. Neppolian, Nanoscale, 7 (2015) 7849-7857.

[302] G. Nagaraju, K. Manjunath, S. Sarkar, E. Gunter, S.R. Teixeira, J. Dupont, Int. J. Hydrogen Energy, 40 (2015) 12209-12216.

[303] M. Wang, K. Han, S. Zhang, L. Sun, Coord. Chem. Rev., 287 (2015) 1-14.

[304] B.L. Greene, C.A. Joseph, M.J. Maroney, R.B. Dyer, J. Am. Chem. Soc., 134 (2012)

11108-11111.

[305] K. Suzuki, F. Tang, Y. Kikukawa, K. Yamaguchi, N. Mizuno, Chem. Lett., 43 (2014) 1429-

1431.

[306] S. Fukuzumi, T. Kobayashi, T. Suenobu, Angew. Chem., Int. Ed., 50 (2011) 728-731, S728/721-S728/728.

[307] H. Lin, D. Liu, J. Long, Z. Zhang, H. Zhuang, Y. Zheng, X. Wang, Phys. Chem. Chem. Phys., 17 (2015) 10726-10736.

[308] M.P. McLaughlin, T.M. McCormick, R. Eisenberg, P.L. Holland, Chem. Commun., 47 (2011) 7989-7991.

[309] A.S. Weingarten, R.V. Kazantsev, L.C. Palmer, M. McClendon, A.R. Koltonow, A.P.S. Samuel, D.J. Kiebala, M.R. Wasielewski, S.I. Stupp, Nat. Chem., 6 (2014) 964-970.

[310] B. Cecconi, N. Manfredi, R. Ruffo, T. Montini, I. Romero-Ocaña, P. Fornasiero, A. Abbotto, ChemSusChem, 8 (2015) 4216-4228.

[311] (a) T.T. Tang, K. Li, Z.M. Shen, T.H. Sun, Y.L. Wang, J.P. Jia, J. Power Sources, 294 (2015) 59-66; (b) P. Lianos, N. Strataki, M. Antoniadou, Pure Appl. Chem., 81 (2009) 1441-1448;

(c) H.G. Cha, K.-S. Choi, Nat. Chem., 7 (2015) 328-333.

[312] (a) D.E. Tsydenov, A.V. Vorontsov, J. Photochem. Photobiol., A, 297 (2015) 8-13; (b) D.E. Tsydenov, V.N. Parmon, A.V. Vorontsov, Int. J. Hydrogen Energy, 37 (2012) 11046-11060.

[313] (a) M. Bowker, H. Bahruji, J. Kennedy, W. Jones, G. Hartley, C. Morton, Catal. Lett., 145 (2015) 214-219; (b) A. Speltini, M. Sturini, D. Dondi, E. Annovazzi, F. Maraschi, V. Caratto, A. Profumo, A. Buttafava, Photochem. Photobiol. Sci., 13 (2014) 1410-1419; (c) K. Lalitha, J.K. 
Reddy, M.V.P. Sharma, V.D. Kumari, M. Subrahmanyam, Int. J. Hydrogen Energy, 35 (2010) 3991-4001; (d) C.K.N. Peh, M. Gao, G.W. Ho, J. Mater. Chem. A, 3 (2015) 19360-19367.

[314] (a) M. Latorre-Sánchez, C. Lavorato, M. Puche, V. Fornés, R. Molinari, H. Garcia, Chem.-Eur. J., 18 (2012) 16774-16783; (b) C. Zhao, H. Luo, F. Chen, P. Zhang, L. Yi, K. You, Energy Environ. Sci., 7 (2014) 1700-1707; (c) V.M. Daskalaki, P. Panagiotopoulou, D.I. Kondarides, Chem. Eng. J., 170 (2011) 433-439.

[315] S. Sato, J. Phys. Chem., 87 (1983) 3531-3537.

[316] N. Strataki, P. Lianos, J. Adv. Oxid. Technol., 11 (2008) 111-115.

[317] D.I. Kondarides, A. Patsoura, X.E. Verykios, J. Adv. Oxid. Technol., 13 (2010) 116-123.

[318] S. Malato, M.I. Maldonado, P. Fernández-Ibáñez, I. Oller, I. Polo, R. Sánchez-Moreno, Mater. Sci. Semicond. Process., (2015).

[319] T. Simon, N. Bouchonville, M.J. Berr, A. Vaneski, A. Adrovic, D. Volbers, R. Wyrwich, M. Doeblinger, A.S. Susha, A.L. Rogach, F. Jaeckel, J.K. Stolarczyk, J. Feldmann, Nat. Mater., 13 (2014) 1013-1018.

[320] V. Lanese, D. Spasiano, R. Marotta, I. Di Somma, L. Lisi, S. Cimino, R. Andreozzi, Int. J. Hydrogen Energy, 38 (2013) 9644-9654.

[321] A. Speltini, M. Sturini, F. Maraschi, D. Dondi, G. Fisogni, E. Annovazzi, A. Profumo, A. Buttafava, Int. J. Hydrogen Energy, 40 (2015) 4303-4310.

[322] A. Reynal, E. Pastor, M.A. Gross, S. Selim, E. Reisner, J.R. Durrant, Chem. Sci., 6 (2015) 4855-4859.

[323] R. Bashiri, N.M. Mohamed, C.F. Kait, S. Sufian, Int. J. Hydrogen Energy, 40 (2015) 6021-

6037.

[324] K. Maeda, Catal. Sci. Technol., 4 (2014) 1949-1953.

[325] A. Speltini, M. Sturini, F. Maraschi, D. Dondi, A. Serra, A. Profumo, A. Buttafava, A. Albini, Int. J. Hydrogen Energy, 39 (2014) 11433-11440.

[326] F. Gärtner, S. Losse, A. Boddien, M.-M. Pohl, S. Denurra, H. Junge, M. Beller, ChemSusChem, 5 (2012) 530-533.

[327] Y. Wu, G. Lu, S. Li, Catal. Lett., 133 (2009) 97-105.

[328] G.L. Chiarello, M.H. Aguirre, E. Selli, J. Catal., 273 (2010) 182-190.

[329] E. Taboada, I. Angurell, J. Llorca, J. Catal., 309 (2014) 460-467.

[330] C.R. López, E.P. Melián, J.A. Ortega Méndez, D.E. Santiago, J.M. Doña Rodríguez, O. González Díaz, J. Photochem. Photobiol., A, 312 (2015) 45-54.

[331] M. Stelmachowski, M. Marchwicka, E. Grabowska, M. Diak, A. Zaleska, J. Adv. Oxid. Technol., 17 (2014) 179-186, 178 pp.

[332] Slamet, D. Tristantini, Valentina, M. Ibadurrohman, Int. J. Energy Res., 37 (2013) $1372-$

1381 .

[333] S. Mozia, A. Heciak, A.W. Morawski, Catal. Today, 161 (2011) 189-195.

[334] X. Jiang, X. Fu, L. Zhang, S. Meng, S. Chen, J. Mater. Chem. A, 3 (2015) 2271-2282.

[335] S. Malato, M.I. Maldonado, P. Fernández-Ibáñez, I. Oller, I. Polo, R. Sánchez-Moreno,

Mater. Sci. Semicond. Process.

[336] D. Praveen Kumar, M.V. Shankar, M. Mamatha Kumari, G. Sadanandam, B. Srinivas, V.

Durgakumari, Chem. Commun., 49 (2013) 9443-9445.

[337] N.H. Tran, G.S.K. Kannangara, Chem. Soc. Rev., 42 (2013) 9454-9479.

[338] Y. Shiraishi, Y. Sugano, S. Tanaka, T. Hirai, Angew. Chem., Int. Ed., 49 (2010) 1656-1660.

[339] C.N. Hamelinck, A.P.C. Faaij, J. Power Sources, 111 (2002) 1-22.

[340] J. Rodriguez, E. Puzenat, R. Mosdale, P.X. Thivel, Fuel Cells, 14 (2014) 403-411.

[341] Y. Sun, J. Cheng, Bioresour Technol, 83 (2002) 1-11.

[342] M.A. Nadeem, M. Murdoch, G.I.N. Waterhouse, J.B. Metson, M.A. Keane, J. Llorca, H. Idriss, J. Photochem. Photobiol., A, 216 (2010) 250-255.

[343] R.R. Soares, D.A. Simonetti, J.A. Dumesic, Angew. Chem., Int. Ed., 45 (2006) 3982-3985. 
[344] M. Stelmachowski, M. Marchwicka, E. Grabowska, M. Diak, A. Zaleska, J. Adv. Oxid. Technol., 17 (2014) 167-178.

[345] P. Panagiotopoulou, E.E. Karamerou, D.I. Kondarides, Catal. Today, 209 (2013) 91-98.

[346] M. Bowker, P.R. Davies, L.S. Al-Mazroai, Catal. Lett., 128 (2009) 253-255.

[347] R. Liu, H. Yoshida, S.-i. Fujita, M. Arai, Appl. Catal., B, 144 (2014) 41-45.

[348] H. Gao, J. Zhang, R. Wang, M. Wang, Appl. Catal., B, 172-173 (2015) 1-6.

[349] (a) B. Ohtani, M. Kakimoto, S. Nishimoto, T. Kagiya, J. Photochem. Photobiol. A-Chem., 70 (1993) 265-272; (b) K. Iseda, T. Osaki, H. Taoda, H. Yamakita, Bull. Chem. Soc. Jpn., 66 (1993) 1038-1042.

[350] (a) D.S. Muggli, S.A. Keyser, J.L. Falconer, Catal. Lett., 55 (1998) 129-132; (b) D.S. Muggli, J.L. Falconer, J. Catal., 187 (1999) 230-237; (c) D.S. Muggli, J.L. Falconer, J. Catal., 191 (2000) 318-325.

[351] B. Kraeutler, A.J. Bard, J. Am. Chem. Soc., 99 (1977) 7729-7731.

[352] A. Chemseddine, H.P. Boehm, J. Mol. Catal., 60 (1990) 295-311.

[353] (a) S. Mozia, A. Heciak, A.W. Morawski, Appl. Catal., B, 104 (2011) 21-29; (b) S. Mozia, A. Heciak, A.W. Morawski, J. Photochem. Photobiol. A-Chem., 216 (2010) 275-282; (c) S. Mozia, A. Heciak, D. Darowna, A.W. Morawski, J. Photochem. Photobiol. A-Chem., 236 (2012) 48-53.

[354] (a) F. Chambon, F. Rataboul, C. Pinel, A. Cabiac, E. Guillon, N. Essayem, Appl. Catal., B, 105 (2011) 171-181; (b) C.-H. Zhou, X. Xia, C.-X. Lin, D.-S. Tong, J. Beltramini, Chem. Soc.

Rev., 40 (2011) 5588-5617.

[355] B. Ohtani, K. Iwai, S.-i. Nishimoto, S. Sato, J. Phys. Chem. B, 101 (1997) 3349-3359.

[356] Y.X. Li, G.X. Lu, S.B. Li, J. Photochem. Photobiol. A-Chem., 152 (2002) 219-228.

[357] M.R. St. John, A.J. Furgala, A.F. Sammells, J. Phys. Chem., 87 (1983) 801-805.

[358] M. Ilie, B. Cojocaru, V.I. Parvulescu, H. Garcia, Int. J. Hydrogen Energy, 36 (2011) 1550915518.

[359] N. Luo, Z. Jiang, H. Shi, F. Cao, T. Xiao, P.P. Edwards, Int. J. Hydrogen Energy, 34 (2009) $125-129$.

[360] H. Fan, G. Li, F. Yang, L. Yang, S. Zhang, J. Chem. Technol. Biotechnol., 86 (2011) 1107-

1112.

[361] I. Dincer, C. Acar, Int. J. Hydrogen Energy, 40 (2015) 11094-11111.

[362] D. Klauson, O. Budarnaja, I.C. Beltran, M. Krichevskaya, S. Preis, Environ. Technol., 35 (2014) 2237-2243.

[363] A. Patsoura, D.I. Kondarides, X.E. Verykios, Appl. Catal., B, 64 (2006) 171-179.

[364] G. Wang, Y. Ling, X. Lu, H. Wang, F. Qian, Y. Tong, Y. Li, Energy Environ. Sci., 5 (2012) 8215-8219.

[365] T. Kida, G. Guan, N. Yamada, T. Ma, K. Kimura, A. Yoshida, Int. J. Hydrogen Energy, 29 (2004) 269-274.

[366] T. Sakata, T. Kawai, K. Hashimoto, Chem. Phys. Lett., 88 (1982) 50-54.

[367] O. Enea, A. Ali, D. Duprez, Int. J. Hydrogen Energy, 13 (1988) 569-572.

[368] Y.Z. Yang, C.H. Chang, H. Idriss, Appl. Catal., B, 67 (2006) 217-222.

[369] A. Galińska, J. Walendziewski, Energy \& Fuels, 19 (2005) 1143-1147.

[370] M. Ikeda, Y. Kusumoto, Y. Yakushijin, S. Somekawa, P. Ngweniform, B. Ahmmad, Catal. Commun., 8 (2007) 1943-1946.

[371] M.V. Shankar, J. Ye, Catal. Commun., 11 (2009) 261-265.

[372] J.-J. Zou, H. He, L. Cui, H.-Y. Du, Int. J. Hydrogen Energy, 32 (2007) 1762-1770.

[373] M. Zalas, M. Laniecki, Solar Energy Materials and Solar Cells, 89 (2005) 287-296.

[374] S. Meyer, S. Saborowski, B. Schäfer, ChemPhysChem, 7 (2006) 572-574.

[375] H. Bahruji, M. Bowker, C. Brookes, P.R. Davies, I. Wawata, Appl. Catal. A-Gen., 454 (2013) 66-73.

[376] Q. Gu, J. Long, H. Zhuang, C. Zhang, Y. Zhou, X. Wang, Phys. Chem. Chem. Phys., 16 (2014) 12521-12534. 
[377] A. Kudo, K. Domen, K. Maruya, T. Onishi, Chem. Phys. Lett., 133 (1987) 517-519.

[378] T. Miwa, S. Kaneco, H. Katsumata, T. Suzuki, K. Ohta, S. Chand Verma, K. Sugihara, Int. J. Hydrogen Energy, 35 (2010) 6554-6560.

[379] S. Mozia, A. Kulagowska, A.W. Morawski, Molecules, 19 (2014) 19633-19647.

[380] M. Ikeda, Y. Kusumoto, S. Somekawa, P. Ngweniform, B. Ahmmad, J. Photochem.

Photobiol., A, 184 (2006) 306-312.

[381] K.M. Parida, S. Martha, D.P. Das, N. Biswal, J. Mater. Chem., 20 (2010) 7144-7149.

[382] L. Amirav, A.P. Alivisatos, J. Phys. Chem. Lett., 1 (2010) 1051-1054.

[383] M.-C. Wu, J.-S. Chih, W.-K. Huang, CrystEngComm, 16 (2014) 10692-10699.

[384] R.M. Navarro, J. Arenales, F. Vaquero, I.D. Gonzalez, J.L.G. Fierro, Catal. Today, 210

(2013) 33-38.

[385] J.J. Zou, C. Chen, C.J. Liu, Y.P. Zhang, Y. Han, L. Cui, Mater. Lett., 59 (2005) 3437-3440.

[386] N. Strataki, N. Boukos, F. Paloukis, S.G. Neophytides, P. Lianos, Photochem. Photobiol.

Sci., 8 (2009) 639-643.

[387] J. Puskelova, R. Michal, M. Caplovicova, M. Antoniadou, L. Caplovic, G. Plesch, P. Lianos, Appl. Surf. Sci., 305 (2014) 665-669.

[388] H. Yuzawa, T. Yoshida, H. Yoshida, Appl. Catal., B, 115 (2012) 294-302.

[389] Y. Mizukoshi, K. Sato, T.J. Konno, N. Masahashi, Appl. Catal., B, 94 (2010) 248-253.

[390] T. Sakata, K. Hashimoto, T. Kawai, J. Phys. Chem., 88 (1984) 5214-5221.

[391] V. Jovic, P.-H. Hsieh, W.-T. Chen, D. Sun-Waterhouse, T. Soehnel, G.I.N. Waterhouse, Int. J. Nanotechnol., 11 (2014) 686-694.

[392] E. Taboada, I. Angurell, J. Llorca, J. Photochem. Photobiol. A-Chem., 281 (2014) 35-39.

[393] Z. Yu, J. Meng, Y. Li, Y. Li, Int. J. Hydrogen Energy, 38 (2013) 16649-16655.

[394] T. Sakata, T. Kawai, Chem. Phys. Lett., 80 (1981) 341-344.

[395] Y.Q. Wu, G.X. Lu, S.B. Li, J. Photochem. Photobiol. A-Chem., 181 (2006) 263-267.

[396] N. Dubey, S.S. Rayalu, N.K. Labhsetwar, S. Devotta, Int. J. Hydrogen Energy, 33 (2008) 5958-5966.

[397] A. Taheri Najafabadi, F. Taghipour, Energy Convers. Manage., 82 (2014) 106-113.

[398] M.I. Rustamov, N.Z. Muradov, A.D. Guseinova, Y.V. Bazhutin, Int. J. Hydrogen Energy, 13 (1988) 533-538.

[399] B.R. Müller, S. Majoni, R. Memming, D. Meissner, J. Phys. Chem. B, 101 (1997) 2501-

2507.

[400] N. Fu, G. Lu, Appl. Surf. Sci., 255 (2009) 4378-4383.

[401] N. Fu, G. Lu, Chem. Commun., (2009) 3591-3593.

[402] N. Fu, G. Lu, Catal. Lett., 127 (2009) 319-322.

[403] J.M. Kum, Y.J. Park, H.J. Kim, S.O. Cho, Nanotechnology, 26 (2015) 125402-125402.

[404] L. Jia, J. Li, W. Fang, J. Alloys Compd., 489 (2009) L13-L16.

[405] S. Shen, L. Guo, Catal. Today, 129 (2007) 414-420.

[406] A. Heciak, A.W. Morawski, B. Grzmil, S. Mozia, Appl. Catal., B, 140 (2013) 108-114.

[407] P. Minh-Hao, D. Cao-Thang, V. Gia-Thanh, T. Ngoc-Don, D. Trong-On, Phys. Chem.

Chem. Phys., 16 (2014) 5937-5941.

[408] H. Tu, L. Xu, F. Mou, J. Guan, Chem. Commun., 51 (2015) 12455-12458.

[409] Y.-x. Pan, H. Zhuang, J. Hong, Z. Fang, H. Liu, B. Liu, Y. Huang, R. Xu, ChemSusChem, 7 (2014) 2537-2544.

[410] J.C. Colmenares, A. Magdziarz, M.A. Aramendia, A. Marinas, J.M. Marinas, F.J. Urbano, J.A. Navio, Catal. Commun., 16 (2011) 1-6.

[411] R.M. Mohamed, E.S. Aazam, Chin. J. Catal., 33 (2012) 247-253. 